\title{
Radiological Dose Calculations For Fusion Facilities
}

\author{
Michael L. Abbott \\ Lee C. Cadwallader \\ David A. Petti
}

April 2003

Idaho National Engineering and Environmental Laboratory Bechtel BWXT Idaho, LLC 
INEEL/EXT-03-00405

\title{
RADIOLOGICAL DOSE CALCULATIONS FOR FUSION FACILITIES
}

\author{
Michael L. Abbott \\ Lee C. Cadwallader \\ David A. Petti \\ Published April 2003 \\ Idaho National Engineering and Environmental Laboratory \\ Idaho Falls, Idaho 83415 \\ Prepared for the \\ U.S. Department of Energy, \\ Idaho Operations Office \\ Under Contract No. DE-AC07-99ID13727
}




\begin{abstract}
This report summarizes the results and rationale for radiological dose calculations for the maximally exposed individual during fusion accident conditions. Early doses per unit activity (Sieverts per TeraBecquerel) are given for 535 magnetic fusion isotopes of interest for several release scenarios. These data can be used for accident assessment calculations to determine if the accident consequences exceed Nuclear Regulatory Commission and Department of Energy evaluation guides. A generalized yearly dose estimate for routine releases, based on 1 Terabecquerel unit releases per radionuclide, has also been performed using averaged site parameters and assumed populations. These routine release data are useful for assessing designs against US Environmental Protection Agency yearly release limits.
\end{abstract}




\section{SUMMARY}

This report gives the results of radiological dose code calculations, normalized by unit activities (sieverts per terabecquerel), for use in assessing public safety consequences of fusion accident scenarios and normal operational releases. The dose code MACCS2 was used to generate the normalized doses for three accident conditions; two atmospheric conditions for a ground level release, and one atmospheric condition for an elevated stack release. Averaged data were used for estimating doses from routine releases. The accident data values are well suited to scoping calculations at early stages of accident analysis, or for safety comparisons between two or more design options. Routine release data are useful for assessing the ability of a design to meet US Environmental Protection Agency yearly release limits. If site information is known for a detailed fusion facility design, then specific accident and normal release calculations should be performed for the site of interest.

Five hundred thirty-five fusion radionuclides were treated in the MACCS2 calculations. Data tables give the equivalent dose in Sv/TBq values in this report for the early, or seven day, dose comprised of cloudshine, groundshine, inhalation during plume passage, inhalation and shine from resuspended particulate that settled from the plume to the ground, and the 50-year adult dose commitment from inhaled radionuclides. The early dose is the immediate concern for public dose consequence, and is the dose that is compared to the evaluation guidelines. When radioactive source terms in terabecquerels are estimated for a fusion off-normal event or accident scenario, the source terms can be multiplied by the table values presented here to obtain the public dose consequence. 


\section{CONTENTS}

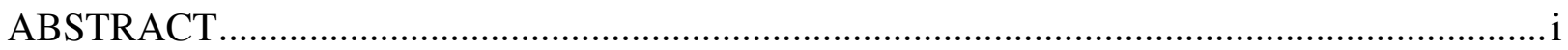

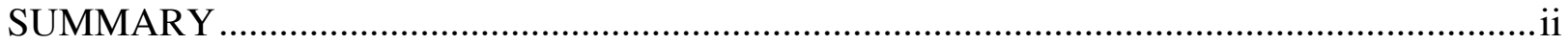

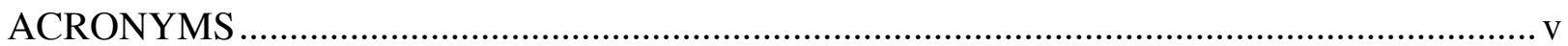

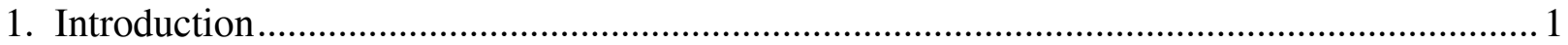

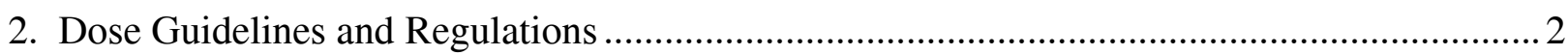

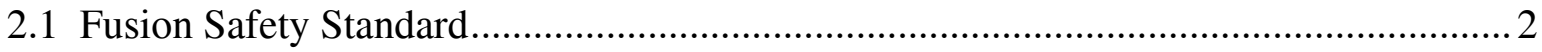

2.2 Code of Federal Regulations .................................................................................. 3

2.3 Deviations from the Fusion Safety Standard..................................................... 4

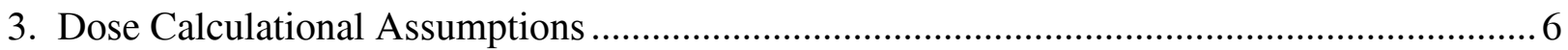

4. Specific Dose Data Tables for Off-Normal Releases ................................................. 7

5. Specific Dose Data Table for Routine Releases ..........................................................50

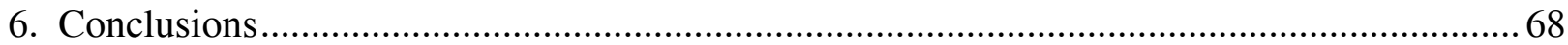

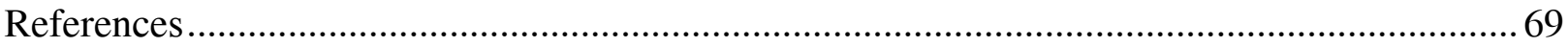




\section{TABLES}

1. FSS requirements for protection of the public from exposure to radiation ......................... 3

2. Early dose results $(\mathrm{Sv} / \mathrm{TBq})$ for a ground-level release at $1 \mathrm{~km}$ downwind distance using stability class D, $4 \mathrm{~m} / \mathrm{s}$ wind speed, no rain, and $\mathrm{P}-\mathrm{G}$ dispersion parameters (sigmas).

3. Early dose results $(\mathrm{Sv} / \mathrm{TBq})$ for a $100-\mathrm{m}$ elevated release at an MEI distance of 2.5 $2.8 \mathrm{~km}$ assuming stability class D, $4 \mathrm{~m} / \mathrm{s}$ wind speed, no rain, and P-G dispersion parameters (sigmas).

4. Early dose results $(\mathrm{Sv} / \mathrm{TBq})$ for a ground-level release at $1 \mathrm{~km}$ downwind distance using stability class F, $1 \mathrm{~m} / \mathrm{s}$ wind speed, no rain, and P-G dispersion parameters (sigmas)

5. Effective dose for annual average releases $(1 \mathrm{TBq} / \mathrm{a})$ of fusion activation products 53 


\section{ACRONYMS}

$\begin{array}{ll}\text { AMAD } & \text { activity median aerodynamic diameter } \\ \text { ARIES } & \text { Advanced Reactor Innovation and Evaluation Study } \\ \text { Bq } & \text { Becquerel (1 disintegration/second) } \\ \text { DOE } & \text { Department of Energy } \\ \text { EDA } & \text { Engineering Design Activity for ITER } \\ \text { EDE } & \text { effective dose equivalent } \\ \text { EPA } & \text { Environmental Protection Agency } \\ \text { EPZ } & \text { Emergency Planning Zone } \\ \text { FSS } & \text { fusion safety standard } \\ \text { ITER } & \text { International Thermonuclear Experimental Reactor } \\ \text { MACCS2 } & \text { MELCOR [melted core] Accident Consequence Code System version } 2 \\ \text { P-G } & \text { Pasquill-Gifford atmospheric stability } \\ \text { MEI } & \text { maximally exposed individual at the site boundary } \\ \text { NRC } & \text { Nuclear Regulatory Commission } \\ \text { NSO } & \text { Next Step Options (fusion design studies for a burning plasma machine } \\ \text { Rv } & \text { Sievert } \\ \text { M } & \end{array}$




\section{RADIOLOGICAL DOSE CALCULATIONS FOR FUSION FACILITIES}

\subsection{INTRODUCTION}

During the International Thermonuclear Experiment Reactor (ITER) Engineering Design Activity (EDA) in the mid-1990's, many postulated accidents were examined for off-site consequences. A need was recognized to have dose calculation tools available to quickly analyze these accident scenarios so that the most consequential events could be determined from the sets of events under examination. Tables of activity normalized radiological doses were created [1,2] using the 'beta test version' of the MELCOR Accident Consequence Code System version 2 (MACCS2). Several release scenarios were considered: elevated stack and ground level release, and stable atmospheric conditions that promoted continued cloud integrity or unstable conditions that promoted cloud dilution in air. These cases were calculated and the results were tabulated for ease of scaling to apply to the Becquerel release amounts from specific ITER accident scenarios. These tables were noted to be useful for US studies as well, including the Next Step Options (NSO) and Advanced Reactor Innovation and Evaluation Study (ARIES) safety evaluations. The tables were modified to use the Pasquill-Gifford (P-G) dispersion model [3] rather than the Klug dispersion model, since the P-G model is more widely accepted in the US, and were recalculated using the published version of MACCS2 [4]. These tables are still valid and in use; MACCS2 is the primary radiological dose code in the US, being funded by the US Nuclear Regulatory Commission (NRC) and the Department of Energy (DOE) [5]. When MACCS2 is upgraded, then the tables may require recalculation. The set of scenarios has been broad enough to encompass most dose calculation needs for conceptual designs that do not have site-specific information. 


\subsection{DOSE GUIDELINES AND REGULATIONS}

Fusion facilities, both operating and planned, are somewhat unique in that they house tritium in fuel quantities (e.g., perhaps kilogram levels) and can also confine a wide array of neutron-activated isotopes. Most of the radioactive dose calculation regulations and guidance address nuclear fission facilities rather than fusion facilities. Fission reactors generally have less tritium and a different set of neutron-activated isotopes of concern. Since fusion radionuclides are rather unique and are driven more by choice of construction materials than by fission of heavy elements, some variation is needed to address the consequence analysis of releases of these radionuclides. This section outlines the regulatory evaluation guidelines applied to fusion facilities.

\subsection{Fusion Safety Standard}

Safety professionals in the magnetic fusion safety community participated in writing a safety document for the DOE that set the precedent for the safety approach at fusion facilities. The Fusion Safety Standard (FSS) [6] was published in 1996, and it contains the guidance for safety design, and evaluation guidelines for public safety of fusion design concepts. The DOE Order on Facility Safety [7] refers to the fusion safety standard as the primary document for achieving safety at fusion facilities. The Order directs that fusion facilities must comply with the fire protection and natural phenomena hazards mitigation portions within the facility safety order, and exempts fusion facilities from the nuclear and explosives safety design criteria, and nuclear criticality safety portions of the order while citing the FSS. Therefore, the FSS is 'called out', or referenced, and carries the regulatory authority of a DOE directive.

The FSS discusses public safety to hazardous and radiological releases. Two sets of radiological criteria shall be used for evaluating radioactive releases: regulatory evaluation guidelines that shall not be exceeded, and fusion requirements. These are given in Table 1. The basis for the fusion values is described in guidance documentation [8]. The regulatory evaluation guidelines are applicable to the maximally exposed individual (MEI) off-site, using conservative calculation assumptions. Best-estimate techniques are used to evaluate against fusion requirements. In showing compliance with these guidelines, the 'as low as reasonably achievable' principle shall be applied. Compliance with both sets of criteria shall be demonstrated for all credible postulated events, noting the difference in analysis methodologies of conservative versus bestestimate calculations. 
Table 1. FSS requirements for protection of the public from exposure to radiation

\begin{tabular}{|l|l|l|}
\hline \multicolumn{1}{|c|}{ Type of release } & $\begin{array}{c}\text { Fusion radiological release } \\
\text { requirement }\end{array}$ & $\begin{array}{c}\text { Regulatory evaluation } \\
\text { guideline }\end{array}$ \\
\hline $\begin{array}{l}\text { Normal and anticipated } \\
\text { operational occurrences }\end{array}$ & $0.1 \mathrm{mSv} / \mathrm{yr}(10 \mathrm{mrem} / \mathrm{yr})$ & $1 \mathrm{mSv} / \mathrm{yr}(100 \mathrm{mrem} / \mathrm{yr})$ \\
\hline $\begin{array}{l}\text { Off-normal conditions, } \\
\text { per event }\end{array}$ & $\begin{array}{l}10 \mathrm{mSv}(1 \mathrm{rem}) \\
\text { [no public evacuation] }\end{array}$ & $250 \mathrm{mSv}(25 \mathrm{rem})$ \\
\hline
\end{tabular}

\subsection{Code of Federal Regulations}

The US Code of Federal Regulations gives an upper bound dose to the public from reactor accident events [9]. The regulation describes that fission power reactors and test reactors must have exclusion areas of such size that an individual [i.e., member of the public] located at any point on its boundary for two hours immediately following onset of the postulated (appreciable) fission product release would not receive a total radiation dose to the whole body in excess of 25 rem or a total radiation dose in excess of 300 rem to the thyroid from iodine exposure. There is further discussion that neither the whole body dose of 25 rem nor the 300 rem value for thyroid exposure as set forth in these site criteria guides are intended to imply that these numbers constitute acceptable limits for emergency doses to the public under accident conditions. Rather, the 25 rem whole body value and the 300 rem thyroid value have been set forth in these guides as reference values, which can be used in the evaluation of reactor sites with respect to potential reactor accidents of exceedingly low probability of occurrence, and low risk of public exposure to radiation.

Radiological dose calculations have always been somewhat conservative, given that there are uncertainties in the calculation process. Early work in accident release dose estimates used class $\mathrm{F}$ atmospheric stability and $1 \mathrm{~m} / \mathrm{s}$ wind speed with a ground level release when site-specific meteorology was unknown, based on NRC guidance $[10,11]$. These " $F$ and 1" atmospheric conditions tend to reduce plume dispersion and dilution, leaving the release plume relatively intact. The intact plume yields a conservatively large early dose value for the public near the site boundary. This conservatism has been condoned within the safety community since dose calculations themselves have sources of error; a Gaussian plume dispersion parameter $(\chi / Q)$ is only an approximation of a very complex phenomenon and could vary by as much as a factor of 2 from a true value [12]. When using conservative weather versus typical weather in dose calculations, factors of 9 or 10 increase in dose can be realized, so the uncertainty in the dispersion parameter becomes less important and the dose is conservatively overestimated with " $F$ and 1". DOE guidance [13] allows use of site-specific weather conditions, as calculated by the NRC [14], but states that $95 \%$ distribution of doses to the maximally exposed offsite individual 
be used as the comparison point to assess against the historic public safety evaluation guideline of $25 \mathrm{rem} / \mathrm{event}$ [9].

\subsection{Deviations from the Fusion Safety Standard}

During preparation for the 2002 Snowmass Fusion Energy Sciences Summer Study (held in July 2002 at Snowmass. Colorado), an important difference was noted with the methodology used to calculate radiological doses, compared to the DOE FSS [6]. As discussed above, the dose calculation assumptions used to compare to the 25 rem $(250 \mathrm{mSv})$ guideline are very conservative; weather conditions that give the least amount of dilution and the highest airborne concentrations of radioactive material (" $\mathrm{F}$ and 1 " in a ground level release). The second requirement is the "no-public evacuation" criteria of $1 \mathrm{rem}(10 \mathrm{mSv})$ per event, taken from Environmental Protection Agency (EPA) guidance [15]. The DOE FSS directs dose calculations using best-estimate techniques, including typical weather conditions and, if applicable, an elevated release height. The precedent for that guidance was taken from past EPA direction. EPA emergency planning dose calculations were performed using best estimates of prevailing weather conditions to most accurately predict the area to be evacuated [15].

Since the publication of the DOE FSS, another office in the DOE has released further guidance on emergency planning. The new DOE guidance directs that at least two sets of atmospheric dispersion conditions be considered in calculating the dose consequence versus distance for each source term at a facility [16]. The first dispersion condition is a 95\% worst-case wind speed and atmospheric stability for the site under consideration, and if these conditions have not been determined for a site, then a $1 \mathrm{~m} / \mathrm{s}$ wind speed and class $\mathrm{F}$ atmospheric stability can be used for an assumed ground level release. These conditions are to be used to develop the size of the emergency planning zone (EPZ). The second set of dispersion conditions should be more typical weather, the average wind speed and most prevalent atmospheric stability class for the site. If these data are not known for a site, class D stability and $4.5 \mathrm{~m} / \mathrm{s}$ wind speed are acceptable assumptions. Used with the results from the conservative case, these typical weather results provide risk perspective about the EPZ and are also useful for offsite planning discussions with local authorities.

This DOE guidance [16] states that conservative weather conditions and an assumed ground level release shall be used to develop the size of the EPZ. For fusion to demonstrate its safety and environmental advantages, public evacuation in the case of any off-site accident release must be shown to be unnecessary. Definitive proof that a public evacuation plan is not needed is to show that the EPZ remains within the facility's site boundary. Therefore, for a fusion facility to prove that it can meet the "no-public evacuation" criteria, the dose calculations for the 1-rem "no-evacuation" evaluation criteria must now be performed assuming conservative weather rather than best estimate weather. The issue of elevated versus ground level release is not as clear for this new guidance. The DOE guidance states an assumed ground level release; presumably this means that actual scenarios can be either elevated or ground, depending on the fault events comprising the scenario. Past regulatory safety work for fusion $[17,18,19,20]$ has 
allowed an elevated release point in the MEI dose calculations when the accident scenario release path included the facility vent stack's elevated release. Events that release through the stack are generally in-plant equipment faults. Some accident scenario release paths are ground level, such as aircraft impact and severe earthquake. Certainly, both elevated and ground level releases must meet the 1-rem criteria.

These new assumptions about weather for dose calculations for the "no evacuation" fusion dose evaluation guide place new restrictions on future fusion facility designs. Future designs should use large site boundaries of $1 \mathrm{~km}$ or more, reduce on-site inventories, and use inventory segmentation, as much as possible, to meet the "noevacuation" guide. 


\subsection{DOSE CALCULATIONAL ASSUMPTIONS}

The tables in this report address the early dose that is incurred during and shortly after the radioactive plume passage. The early dose includes the contributions from cloudshine (external exposure to radioactivity in the plume) during plume passage, seven days of groundshine (external exposure to radioactive material on the ground from wet or dry deposition processes), and the 50-year adult dose commitment to human body organs due to inhalation during plume passage and seven days of particle resuspension from the ground [3]. These dose contributions comprise the immediate threat to people [8]. The early dose to the MEI is the value to be compared to evaluation guidelines from Table 1. While the FSS guidance document states that only the plume passage dose should be compared to evaluation guidelines [8], the plume passage dose implicitly assumes public evacuation to preclude exposure to groundshine and any resuspended particles from the ground. A true comparison to the 1-rem no-evacuation guideline means evaluating the complete early dose that could be received (that is, including the groundshine and resuspension contributions) to determine if the public must be evacuated to avoid an early dose of 1 rem or greater. It is unlikely that the first several hours of plume passage can be avoided by evacuation, so the plume passage dose is suggested as a comparison to other evaluation guidelines.

The assumptions used to generate the data tables are as follows:

Two ground level release cases were used, P-G stability class D and $4 \mathrm{~m} / \mathrm{s}$ wind speed, and P-G stability class F with $1 \mathrm{~m} / \mathrm{s}$ wind speed. Both are for a 1-kilometer receptor distance, and evaluate the MEI at $1 \mathrm{~km}$. The MEI distance for the 100-m elevated release was determined in a MACCS2 run as the distance with the maximum normalized air concentration for a non-decaying isotope with no plume depletion effects. That distance was 2.5 to $2.8 \mathrm{~km}$.

All radionuclides are assumed to be in the oxide form and have an activity mean aerodynamic diameter of 1 micron. The source term release duration was 1 hour. For elevated releases, the stack height is assumed to be $100-\mathrm{m}, 2.5$ times higher than the building height. The other building dimensions were assumed to be $40 \mathrm{~m}$ by $100 \mathrm{~m}$. No building wake effects were considered since this stack height is above the building wake zone. The particulate deposition velocity was chosen to be $0.002 \mathrm{~m} / \mathrm{s}$, which is appropriate for 1-micron particles [21]. The calculation model input values for the P-G dispersion 'sigma' parameters were default MACCS2 values as summarized by Tadmor [22]. 


\subsection{SPECIFIC DOSE DATA TABLES FOR OFF-NORMAL RELEASES}

This chapter presents the tables of doses for the 535 fusion radionuclides. These tables are large, and may be more easily handled using a spreadsheet or computer program than by hand calculation. Table 2 gives the MEI early dose results for a ground-level release with class D atmospheric stability and $4 \mathrm{~m} / \mathrm{s}$ wind speed. Table 3 gives the MEI dose results for a 100-m elevated stack release. The MEI distance of 2.5 to $2.8 \mathrm{~km}$ for the $100-\mathrm{m}$ elevated release was determined for a non-decaying isotope with no plume depletion effects; that is, an average case rather than performing hundreds of calculations for each radionuclide to determine the maximum dose distance for each radionuclide. Because of isotope-specific in-transit decay, deposition rate, and plume depletion effects, the MEI distance of 2.5 to $2.8 \mathrm{~km}$ may vary slightly from the distance with the highest dose [3]. Table 4 gives the MEI dose for a ground-level release using P-G Class F atmospheric stability and $1 \mathrm{~m} / \mathrm{s}$ wind speed. 
Table 2. Early dose results (Sv/TBq) for a ground-level release at $1 \mathrm{~km}$ downwind distance using stability class $D, 4 \mathrm{~m} / \mathrm{s}$ wind speed, no rain, and $P-G$ dispersion parameters (sigmas).

\begin{tabular}{|c|c|c|c|c|c|c|}
\hline Nuclide & $\begin{array}{c}\text { 7-day } \\
\text { Total } \\
(1+2+3+4)\end{array}$ & $\begin{array}{c}\text { Cloud } \\
\text { shine } \\
(1)\end{array}$ & $\begin{array}{l}\text { Ground } \\
\text { shine } \\
(2)\end{array}$ & $\begin{array}{c}\text { Inhalation } \\
(3)\end{array}$ & $\begin{array}{l}\text { Resus- } \\
\text { pension } \\
(4)\end{array}$ & $\begin{array}{c}\text { Plume } \\
\text { Passage } \\
(1)+(3)\end{array}$ \\
\hline $\mathrm{H}-3$ & $1.71 \mathrm{E}-07$ & $0.00 \mathrm{E}+00$ & $0.00 \mathrm{E}+00$ & $8.85 \mathrm{E}-08$ & $8.27 \mathrm{E}-08$ & $8.85 \mathrm{E}-08$ \\
\hline $\mathrm{Be}-7$ & $1.20 \mathrm{E}-06$ & $1.45 \mathrm{E}-08$ & 7.92E-07 & 3.73E-07 & $1.71 \mathrm{E}-08$ & $3.88 \mathrm{E}-07$ \\
\hline $\mathrm{Be}-10$ & 3.33E-04 & $0.00 \mathrm{E}+00$ & $0.00 \mathrm{E}+00$ & $3.18 \mathrm{E}-04$ & $1.50 \mathrm{E}-05$ & $3.18 \mathrm{E}-04$ \\
\hline C-11 & $3.41 \mathrm{E}-08$ & $3.41 \mathrm{E}-08$ & $0.00 \mathrm{E}+00$ & $0.00 \mathrm{E}+00$ & $0.00 \mathrm{E}+00$ & $3.41 \mathrm{E}-08$ \\
\hline C-14 & 3.31E-08 & $0.00 \mathrm{E}+00$ & $0.00 \mathrm{E}+00$ & 3.31E-08 & $0.00 \mathrm{E}+00$ & 3.31E-08 \\
\hline $\mathrm{N}-13$ & 3.76E-09 & $3.48 \mathrm{E}-09$ & $0.00 \mathrm{E}+00$ & $2.78 \mathrm{E}-10$ & $0.00 \mathrm{E}+00$ & 3.76E-09 \\
\hline $0-15$ & $9.98 \mathrm{E}-17$ & $9.98 \mathrm{E}-17$ & $0.00 \mathrm{E}+00$ & $0.00 \mathrm{E}+00$ & $0.00 \mathrm{E}+00$ & $9.98 \mathrm{E}-17$ \\
\hline $\mathrm{N}-16$ & $0.00 \mathrm{E}+00$ & $0.00 \mathrm{E}+00$ & $0.00 \mathrm{E}+00$ & $0.00 \mathrm{E}+00$ & $0.00 \mathrm{E}+00$ & $0.00 \mathrm{E}+00$ \\
\hline F-18 & 3.53E-06 & $1.57 \mathrm{E}-07$ & $3.32 \mathrm{E}-06$ & 5.50E-08 & $2.00 \mathrm{E}-09$ & $2.12 \mathrm{E}-07$ \\
\hline $\mathrm{Na}-22$ & 4.34E-05 & $6.50 \mathrm{E}-07$ & 3.36E-05 & 8.82E-06 & $4.15 \mathrm{E}-07$ & 9.47E-06 \\
\hline $\mathrm{Na}-24$ & $9.78 \mathrm{E}-06$ & $1.33 \mathrm{E}-06$ & 7.17E-06 & 1.27E-06 & $1.53 \mathrm{E}-08$ & $2.60 \mathrm{E}-06$ \\
\hline$M g-27$ & 3.32E-09 & $2.48 \mathrm{E}-09$ & $5.61 \mathrm{E}-10$ & $2.76 \mathrm{E}-10$ & $4.51 \mathrm{E}-14$ & 2.76E-09 \\
\hline Mg-28 & 1.56E-05 & $9.55 \mathrm{E}-07$ & $8.08 \mathrm{E}-06$ & $6.46 \mathrm{E}-06$ & $9.92 \mathrm{E}-08$ & 7.42E-06 \\
\hline Al-26 & 1.30E-04 & $8.49 \mathrm{E}-07$ & 4.01E-05 & 8.50E-05 & 4.01E-06 & 8.58E-05 \\
\hline Al-28 & $1.59 \mathrm{E}-15$ & $1.38 \mathrm{E}-15$ & $1.81 \mathrm{E}-16$ & $3.17 \mathrm{E}-17$ & $0.00 \mathrm{E}+00$ & $1.41 \mathrm{E}-15$ \\
\hline Si-31 & 2.76E-07 & $2.01 \mathrm{E}-10$ & $2.47 \mathrm{E}-10$ & $2.75 \mathrm{E}-07$ & $7.11 \mathrm{E}-10$ & $2.75 \mathrm{E}-07$ \\
\hline Si-32 & $6.14 \mathrm{E}-05$ & $0.00 \mathrm{E}+00$ & $0.00 \mathrm{E}+00$ & $5.86 \mathrm{E}-05$ & $2.79 \mathrm{E}-06$ & 5.86E-05 \\
\hline S-37 & $2.90 \mathrm{E}-12$ & $0.00 \mathrm{E}+00$ & $0.00 \mathrm{E}+00$ & $2.90 \mathrm{E}-12$ & $2.53 \mathrm{E}-16$ & $2.90 \mathrm{E}-12$ \\
\hline P-32 & 5.95E-06 & $0.00 \mathrm{E}+00$ & $0.00 \mathrm{E}+00$ & 5.71E-06 & $2.42 \mathrm{E}-07$ & 5.71E-06 \\
\hline P-33 & 6.33E-07 & $0.00 \mathrm{E}+00$ & $0.00 \mathrm{E}+00$ & 6.06E-07 & $2.69 \mathrm{E}-08$ & 6.06E-07 \\
\hline S-35 & $3.52 \mathrm{E}-07$ & $0.00 \mathrm{E}+00$ & $0.00 \mathrm{E}+00$ & 3.37E-07 & $1.56 \mathrm{E}-08$ & 3.37E-07 \\
\hline $\mathrm{Cl}-36$ & 3.76E-06 & $2.02 \mathrm{E}-15$ & $1.21 \mathrm{E}-11$ & 1.94E-06 & $1.82 \mathrm{E}-06$ & 1.94E-06 \\
\hline $\mathrm{Cl}-38$ & 1.04E-06 & $1.23 \mathrm{E}-07$ & 8.65E-07 & 4.85E-08 & $6.12 \mathrm{E}-10$ & 1.72E-07 \\
\hline Cl-39 & $5.75 \mathrm{E}-08$ & $0.00 \mathrm{E}+00$ & $0.00 \mathrm{E}+00$ & 5.65E-08 & 1.06E-09 & 5.65E-08 \\
\hline $\mathrm{Cl}-40$ & $4.57 \mathrm{E}-23$ & $0.00 \mathrm{E}+00$ & $0.00 \mathrm{E}+00$ & 4.57E-23 & $0.00 \mathrm{E}+00$ & $4.57 \mathrm{E}-23$ \\
\hline Ar-37 & $4.00 \mathrm{E}-13$ & $4.00 \mathrm{E}-13$ & $0.00 \mathrm{E}+00$ & $0.00 \mathrm{E}+00$ & $0.00 \mathrm{E}+00$ & $4.00 \mathrm{E}-13$ \\
\hline Ar-39 & $0.00 \mathrm{E}+00$ & $0.00 \mathrm{E}+00$ & $0.00 \mathrm{E}+00$ & $0.00 \mathrm{E}+00$ & $0.00 \mathrm{E}+00$ & $0.00 \mathrm{E}+00$ \\
\hline$A r-41$ & 2.61E-07 & $2.61 \mathrm{E}-07$ & $0.00 \mathrm{E}+00$ & $0.00 \mathrm{E}+00$ & $0.00 \mathrm{E}+00$ & 2.61E-07 \\
\hline $\mathrm{K}-40$ & 1.79E-05 & $4.73 \mathrm{E}-08$ & $2.19 \mathrm{E}-06$ & $1.50 \mathrm{E}-05$ & 7.06E-07 & $1.50 \mathrm{E}-05$ \\
\hline $\mathrm{K}-42$ & 1.93E-06 & 7.94E-08 & $4.00 \mathrm{E}-07$ & 1.43E-06 & $1.48 \mathrm{E}-08$ & $1.51 \mathrm{E}-06$ \\
\hline $\mathrm{K}-43$ & $4.14 \mathrm{E}-06$ & $2.78 \mathrm{E}-07$ & $3.09 \mathrm{E}-06$ & 7.61E-07 & $1.23 \mathrm{E}-08$ & 1.04E-06 \\
\hline Ca-41 & $1.41 \mathrm{E}-06$ & $9.19 \mathrm{E}-13$ & $3.32 \mathrm{E}-10$ & $1.35 \mathrm{E}-06$ & $6.36 \mathrm{E}-08$ & 1.35E-06 \\
\hline Ca-45 & 7.82E-06 & $4.78 \mathrm{E}-18$ & $3.39 \mathrm{E}-15$ & 7.47E-06 & $3.49 \mathrm{E}-07$ & 7.47E-06 \\
\hline Ca-47 & 1.84E-05 & $3.18 \mathrm{E}-07$ & 1.01E-05 & $7.68 \mathrm{E}-06$ & 2.93E-07 & 8.00E-06 \\
\hline Ca-49 & $2.47 \mathrm{E}-08$ & $6.90 \mathrm{E}-09$ & 1.08E-09 & 1.67E-08 & $1.64 \mathrm{E}-11$ & $2.36 \mathrm{E}-08$ \\
\hline Sc-44 & 1.03E-05 & $4.78 \mathrm{E}-07$ & $9.25 \mathrm{E}-06$ & 5.84E-07 & $2.23 \mathrm{E}-08$ & 1.06E-06 \\
\hline Sc-44m & 1.39E-04 & $9.72 \mathrm{E}-08$ & $1.29 \mathrm{E}-04$ & 7.69E-06 & $2.30 \mathrm{E}-06$ & 7.79E-06 \\
\hline Sc-46 & $3.20 \mathrm{E}-04$ & $5.55 \mathrm{E}-07$ & $2.75 \mathrm{E}-04$ & 3.00E-05 & $1.40 \mathrm{E}-05$ & 3.06E-05 \\
\hline
\end{tabular}


Table 2. Ground release, Class D, $4 \mathrm{~m} / \mathrm{s}$ wind, $1 \mathrm{~km}$, no rain, continued.

\begin{tabular}{|c|c|c|c|c|c|c|}
\hline Nuclide & $\begin{array}{c}\text { 7-day } \\
\text { Total } \\
(1+2+3+4)\end{array}$ & $\begin{array}{c}\text { Cloud } \\
\text { shine } \\
(1)\end{array}$ & $\begin{array}{l}\text { Ground } \\
\text { shine } \\
(2)\end{array}$ & $\begin{array}{c}\text { Inhalation } \\
\text { (3) }\end{array}$ & $\begin{array}{c}\text { Resus- } \\
\text { pension } \\
(4)\end{array}$ & $\begin{array}{c}\text { Plume } \\
\text { Passage } \\
(1)+(3)\end{array}$ \\
\hline Sc-47 & $1.24 \mathrm{E}-05$ & $2.96 \mathrm{E}-08$ & $9.64 \mathrm{E}-06$ & $2.08 \mathrm{E}-06$ & $6.59 \mathrm{E}-07$ & $2.11 \mathrm{E}-06$ \\
\hline Sc-48 & 1.66E-04 & $9.05 \mathrm{E}-07$ & 1.60E-04 & $4.50 \mathrm{E}-06$ & 1.10E-06 & $5.41 \mathrm{E}-06$ \\
\hline Sc-49 & 8.69E-08 & $1.40 \mathrm{E}-10$ & $6.99 \mathrm{E}-10$ & $8.52 \mathrm{E}-08$ & $8.38 \mathrm{E}-10$ & $8.53 \mathrm{E}-08$ \\
\hline Sc-50 & 7.36E-20 & $0.00 \mathrm{E}+00$ & $0.00 \mathrm{E}+00$ & 7.36E-20 & $0.00 \mathrm{E}+00$ & 7.36E-20 \\
\hline $\mathrm{Ti}-44$ & 5.86E-04 & 1.34E-07 & $3.19 \mathrm{E}-04$ & $1.81 \mathrm{E}-04$ & $8.61 \mathrm{E}-05$ & $1.81 \mathrm{E}-04$ \\
\hline Ti-45 & 3.47E-06 & $1.81 \mathrm{E}-07$ & $3.05 \mathrm{E}-06$ & $2.37 \mathrm{E}-07$ & 7.21E-09 & $4.18 \mathrm{E}-07$ \\
\hline Ti-51 & $6.86 \mathrm{E}-11$ & $4.83 \mathrm{E}-11$ & $1.06 \mathrm{E}-11$ & $9.72 \mathrm{E}-12$ & $9.67 \mathrm{E}-16$ & $5.80 \mathrm{E}-11$ \\
\hline$V-48$ & $3.65 \mathrm{E}-04$ & $7.98 \mathrm{E}-07$ & $3.50 \mathrm{E}-04$ & 1.02E-05 & 4.41E-06 & 1.10E-05 \\
\hline$V-49$ & 5.17E-07 & $2.49 \mathrm{E}-12$ & 1.10E-08 & $3.43 \mathrm{E}-07$ & $1.62 \mathrm{E}-07$ & $3.43 \mathrm{E}-07$ \\
\hline V-52 & $3.72 \mathrm{E}-12$ & $3.09 \mathrm{E}-12$ & $5.05 \mathrm{E}-13$ & $1.30 \mathrm{E}-13$ & $8.40 \mathrm{E}-18$ & $3.22 \mathrm{E}-12$ \\
\hline V-53 & 7.96E-21 & $0.00 \mathrm{E}+00$ & $0.00 \mathrm{E}+00$ & 7.96E-21 & $0.00 \mathrm{E}+00$ & $7.96 \mathrm{E}-21$ \\
\hline $\mathrm{Cr}-49$ & 2.04E-07 & 1.07E-07 & $5.51 \mathrm{E}-08$ & $4.20 \mathrm{E}-08$ & $3.18 \mathrm{E}-11$ & 1.49E-07 \\
\hline Cr-51 & $9.05 \mathrm{E}-07$ & $9.24 \mathrm{E}-09$ & $5.02 \mathrm{E}-07$ & 3.77E-07 & $1.68 \mathrm{E}-08$ & 3.86E-07 \\
\hline Mn-52 & 4.30E-05 & 1.04E-06 & $3.51 \mathrm{E}-05$ & $6.57 \mathrm{E}-06$ & $2.40 \mathrm{E}-07$ & 7.61E-06 \\
\hline Mn-52m & 1.31E-07 & $8.76 \mathrm{E}-08$ & $2.82 \mathrm{E}-08$ & 1.49E-08 & 1.63E-11 & 1.03E-07 \\
\hline Mn-53 & 5.90E-07 & $5.11 \mathrm{E}-12$ & $2.54 \mathrm{E}-09$ & 5.61E-07 & $2.65 \mathrm{E}-08$ & 5.61E-07 \\
\hline Mn-54 & 2.17E-05 & $2.55 \mathrm{E}-07$ & $1.34 \mathrm{E}-05$ & 7.65E-06 & $3.59 \mathrm{E}-07$ & 7.91E-06 \\
\hline Mn-56 & 1.34E-06 & 4.07E-07 & 4.87E-07 & 4.43E-07 & 1.13E-09 & 8.50E-07 \\
\hline Mn-57 & $1.54 \mathrm{E}-21$ & 1.03E-21 & $1.70 \mathrm{E}-22$ & 3.37E-22 & $0.00 \mathrm{E}+00$ & 1.37E-21 \\
\hline Fe-52 & 4.33E-06 & 7.95E-07 & $3.44 \mathrm{E}-06$ & $1.00 \mathrm{E}-07$ & 1.07E-09 & 8.95E-07 \\
\hline $\mathrm{Fe}-55$ & $1.51 \mathrm{E}-06$ & $6.73 \mathrm{E}-12$ & $3.55 \mathrm{E}-09$ & $1.44 \mathrm{E}-06$ & $6.77 \mathrm{E}-08$ & 1.44E-06 \\
\hline Fe-59 & $3.18 \mathrm{E}-05$ & $3.59 \mathrm{E}-07$ & $1.66 \mathrm{E}-05$ & 1.42E-05 & $6.45 \mathrm{E}-07$ & 1.46E-05 \\
\hline Fe-60 & 3.31E-04 & $1.28 \mathrm{E}-09$ & $1.21 \mathrm{E}-07$ & $3.16 \mathrm{E}-04$ & $1.49 \mathrm{E}-05$ & $3.16 \mathrm{E}-04$ \\
\hline Co-56 & $5.27 \mathrm{E}-05$ & 1.16E-06 & $5.15 \mathrm{E}-05$ & $0.00 \mathrm{E}+00$ & $0.00 \mathrm{E}+00$ & 1.16E-06 \\
\hline Co-57 & 1.27E-05 & 3.69E-08 & $2.27 \mathrm{E}-06$ & 9.96E-06 & 4.67E-07 & 1.00E-05 \\
\hline Co-58 & $2.84 \mathrm{E}-05$ & $2.95 \mathrm{E}-07$ & $1.53 \mathrm{E}-05$ & 1.23E-05 & $5.67 \mathrm{E}-07$ & $1.26 \mathrm{E}-05$ \\
\hline Co-58m & 1.99E-07 & $1.33 \mathrm{E}-10$ & $7.68 \mathrm{E}-08$ & 1.19E-07 & $3.49 \mathrm{E}-09$ & 1.19E-07 \\
\hline Co-60 & $2.89 \mathrm{E}-04$ & $7.55 \mathrm{E}-07$ & $3.63 \mathrm{E}-05$ & $2.41 \mathrm{E}-04$ & $1.13 \mathrm{E}-05$ & $2.42 \mathrm{E}-04$ \\
\hline Co-60m & 1.15E-09 & $2.12 \mathrm{E}-11$ & $1.42 \mathrm{E}-10$ & $9.44 \mathrm{E}-10$ & $4.27 \mathrm{E}-11$ & $9.65 \mathrm{E}-10$ \\
\hline Co-61 & 1.64E-07 & $1.63 \mathrm{E}-08$ & $1.78 \mathrm{E}-08$ & $1.29 \mathrm{E}-07$ & $2.14 \mathrm{E}-10$ & 1.45E-07 \\
\hline Co-62m & $2.99 \mathrm{E}-09$ & $0.00 \mathrm{E}+00$ & $0.00 \mathrm{E}+00$ & 2.99E-09 & $7.17 \mathrm{E}-13$ & 2.99E-09 \\
\hline $\mathrm{Ni}-56$ & $2.11 \mathrm{E}-05$ & $5.18 \mathrm{E}-07$ & $2.05 \mathrm{E}-05$ & $0.00 \mathrm{E}+00$ & $0.00 \mathrm{E}+00$ & $5.18 \mathrm{E}-07$ \\
\hline $\mathrm{Ni}-57$ & 1.12E-05 & $5.74 \mathrm{E}-07$ & 8.36E-06 & $2.22 \mathrm{E}-06$ & 4.96E-08 & 2.79E-06 \\
\hline $\mathrm{Ni}-59$ & $1.00 \mathrm{E}-06$ & $1.13 \mathrm{E}-11$ & $6.70 \mathrm{E}-09$ & $9.52 \mathrm{E}-07$ & $4.49 \mathrm{E}-08$ & $9.52 \mathrm{E}-07$ \\
\hline $\mathrm{Ni}-63$ & $2.74 \mathrm{E}-06$ & $0.00 \mathrm{E}+00$ & $0.00 \mathrm{E}+00$ & 2.61E-06 & $1.23 \mathrm{E}-07$ & 2.61E-06 \\
\hline $\mathrm{Ni}-65$ & 5.64E-07 & $1.24 \mathrm{E}-07$ & $1.46 \mathrm{E}-07$ & $2.94 \mathrm{E}-07$ & $7.33 \mathrm{E}-10$ & $4.18 \mathrm{E}-07$ \\
\hline Cu-61 & 5.48E-07 & 1.95E-07 & $3.53 \mathrm{E}-07$ & $0.00 \mathrm{E}+00$ & $0.00 \mathrm{E}+00$ & 1.95E-07 \\
\hline Cu-62 & 4.47E-09 & 3.07E-09 & $7.63 \mathrm{E}-10$ & $6.42 \mathrm{E}-10$ & $1.08 \mathrm{E}-13$ & 3.71E-09 \\
\hline Cu-64 & 7.91E-07 & $5.23 \mathrm{E}-08$ & $3.31 \mathrm{E}-07$ & 4.04E-07 & $4.25 \mathrm{E}-09$ & 4.56E-07 \\
\hline Cu-66 & $3.89 \mathrm{E}-12$ & $0.00 \mathrm{E}+00$ & $0.00 \mathrm{E}+00$ & $3.89 \mathrm{E}-12$ & $3.43 \mathrm{E}-16$ & $3.89 \mathrm{E}-12$ \\
\hline Cu-67 & 9.79E-07 & $3.40 \mathrm{E}-08$ & $9.45 \mathrm{E}-07$ & $0.00 \mathrm{E}+00$ & $0.00 \mathrm{E}+00$ & $3.40 \mathrm{E}-08$ \\
\hline
\end{tabular}


Table 2. Ground release, Class D, $4 \mathrm{~m} / \mathrm{s}$ wind, $1 \mathrm{~km}$, no rain, continued.

\begin{tabular}{|c|c|c|c|c|c|c|}
\hline Nuclide & $\begin{array}{c}\text { 7-day } \\
\text { Total } \\
(1+2+3+4)\end{array}$ & $\begin{array}{l}\text { Cloud } \\
\text { shine } \\
(1)\end{array}$ & $\begin{array}{c}\text { Ground } \\
\text { shine } \\
(2)\end{array}$ & $\begin{array}{c}\text { Inhalation } \\
(3)\end{array}$ & $\begin{array}{l}\text { Resus- } \\
\text { pension } \\
(4)\end{array}$ & $\begin{array}{c}\text { Plume } \\
\text { Passage } \\
(1)+(3)\end{array}$ \\
\hline $\mathrm{Zn}-62$ & 2.33E-06 & 3.96E-07 & $1.88 \mathrm{E}-06$ & $5.74 \mathrm{E}-08$ & $4.72 \mathrm{E}-10$ & $4.53 \mathrm{E}-07$ \\
\hline $\mathrm{Zn}-63$ & $5.26 \mathrm{E}-08$ & $0.00 \mathrm{E}+00$ & $0.00 \mathrm{E}+00$ & $5.26 \mathrm{E}-08$ & $3.42 \mathrm{E}-11$ & $5.26 \mathrm{E}-08$ \\
\hline Zn-65 & $3.27 \mathrm{E}-05$ & 1.76E-07 & 8.69E-06 & $2.28 \mathrm{E}-05$ & 1.07E-06 & $2.30 \mathrm{E}-05$ \\
\hline Zn-69 & $3.58 \mathrm{E}-08$ & $8.02 \mathrm{E}-13$ & $5.20 \mathrm{E}-13$ & $3.58 \mathrm{E}-08$ & $3.47 \mathrm{E}-11$ & $3.58 \mathrm{E}-08$ \\
\hline $\mathrm{Zn}-69 \mathrm{~m}$ & $2.06 \mathrm{E}-06$ & $1.15 \mathrm{E}-07$ & $8.06 \mathrm{E}-07$ & 1.13E-06 & $1.30 \mathrm{E}-08$ & $1.25 \mathrm{E}-06$ \\
\hline $\mathrm{Zn}-71 \mathrm{~m}$ & 5.54E-07 & $0.00 \mathrm{E}+00$ & $0.00 \mathrm{E}+00$ & $5.51 \mathrm{E}-07$ & $2.08 \mathrm{E}-09$ & $5.51 \mathrm{E}-07$ \\
\hline Ga-66 & $3.54 \mathrm{E}-06$ & $7.58 \mathrm{E}-07$ & $2.79 \mathrm{E}-06$ & $0.00 \mathrm{E}+00$ & $0.00 \mathrm{E}+00$ & $7.58 \mathrm{E}-07$ \\
\hline Ga-67 & 1.40E-06 & $4.25 \mathrm{E}-08$ & $1.36 \mathrm{E}-06$ & $0.00 \mathrm{E}+00$ & $0.00 \mathrm{E}+00$ & $4.25 \mathrm{E}-08$ \\
\hline Ga-68 & $3.54 \mathrm{E}-07$ & $1.45 \mathrm{E}-07$ & $1.04 \mathrm{E}-07$ & $1.05 \mathrm{E}-07$ & $1.21 \mathrm{E}-10$ & $2.50 \mathrm{E}-07$ \\
\hline Ga-70 & 6.86E-09 & $0.00 \mathrm{E}+00$ & $0.00 \mathrm{E}+00$ & 6.86E-09 & $2.49 \mathrm{E}-12$ & $6.86 \mathrm{E}-09$ \\
\hline Ga-72 & $8.04 \mathrm{E}-06$ & $8.33 \mathrm{E}-07$ & $4.81 \mathrm{E}-06$ & $2.37 \mathrm{E}-06$ & $2.71 \mathrm{E}-08$ & $3.20 \mathrm{E}-06$ \\
\hline Ge-68 & 1.57E-05 & $1.34 \mathrm{E}-07$ & $1.55 \mathrm{E}-05$ & $9.67 \mathrm{E}-08$ & $9.32 \mathrm{E}-09$ & $2.31 \mathrm{E}-07$ \\
\hline Ge-69 & 8.34E-07 & $0.00 \mathrm{E}+00$ & $0.00 \mathrm{E}+00$ & $8.16 \mathrm{E}-07$ & 1.86E-08 & $8.16 \mathrm{E}-07$ \\
\hline Ge-71 & $1.55 \mathrm{E}-08$ & $2.67 \mathrm{E}-11$ & $1.54 \mathrm{E}-08$ & $0.00 \mathrm{E}+00$ & $0.00 \mathrm{E}+00$ & $2.67 \mathrm{E}-11$ \\
\hline Ge-75 & 7.99E-08 & $0.00 \mathrm{E}+00$ & $0.00 \mathrm{E}+00$ & $7.98 \mathrm{E}-08$ & $1.11 \mathrm{E}-10$ & $7.98 \mathrm{E}-08$ \\
\hline Ge-77 & 3.57E-06 & $3.00 \mathrm{E}-07$ & $1.65 \mathrm{E}-06$ & $1.61 \mathrm{E}-06$ & $2.24 \mathrm{E}-08$ & $1.91 \mathrm{E}-06$ \\
\hline As-72 & $6.84 \mathrm{E}-06$ & $5.22 \mathrm{E}-07$ & $6.32 \mathrm{E}-06$ & $0.00 \mathrm{E}+00$ & $0.00 \mathrm{E}+00$ & $5.22 \mathrm{E}-07$ \\
\hline As-73 & $1.51 \mathrm{E}-07$ & 1.32E-09 & $1.50 \mathrm{E}-07$ & $0.00 \mathrm{E}+00$ & $0.00 \mathrm{E}+00$ & $1.32 \mathrm{E}-09$ \\
\hline As-74 & $2.05 \mathrm{E}-05$ & $2.26 \mathrm{E}-07$ & $1.11 \mathrm{E}-05$ & $8.88 \mathrm{E}-06$ & $3.84 \mathrm{E}-07$ & $9.11 \mathrm{E}-06$ \\
\hline As-76 & $6.39 \mathrm{E}-06$ & $1.25 \mathrm{E}-07$ & $1.51 \mathrm{E}-06$ & 4.66E-06 & 8.37E-08 & $4.79 \mathrm{E}-06$ \\
\hline As-77 & $1.38 \mathrm{E}-06$ & $2.49 \mathrm{E}-09$ & $4.69 \mathrm{E}-08$ & $1.30 \mathrm{E}-06$ & $2.95 \mathrm{E}-08$ & $1.30 \mathrm{E}-06$ \\
\hline As-78 & 3.04E-07 & $0.00 \mathrm{E}+00$ & $0.00 \mathrm{E}+00$ & 3.03E-07 & $4.63 \mathrm{E}-10$ & 3.03E-07 \\
\hline Se-73 & 1.37E-06 & $2.87 \mathrm{E}-07$ & $1.08 \mathrm{E}-06$ & $0.00 \mathrm{E}+00$ & $0.00 \mathrm{E}+00$ & 2.87E-07 \\
\hline Se-75 & 1.69E-05 & $1.15 \mathrm{E}-07$ & $6.78 \mathrm{E}-06$ & $9.53 \mathrm{E}-06$ & 4.43E-07 & $9.65 \mathrm{E}-06$ \\
\hline Se-79 & 1.12E-05 & $0.00 \mathrm{E}+00$ & $0.00 \mathrm{E}+00$ & 1.07E-05 & $5.06 \mathrm{E}-07$ & $1.07 \mathrm{E}-05$ \\
\hline $\mathrm{Br}-77$ & $3.62 \mathrm{E}-05$ & $7.48 \mathrm{E}-08$ & $3.61 \mathrm{E}-05$ & $0.00 \mathrm{E}+00$ & $0.00 \mathrm{E}+00$ & $7.48 \mathrm{E}-08$ \\
\hline $\mathrm{Br}-80$ & 1.26E-08 & 1.40E-09 & 8.46E-09 & 2.69E-09 & $1.60 \mathrm{E}-11$ & $4.09 \mathrm{E}-09$ \\
\hline $\mathrm{Br}-80 \mathrm{~m}$ & 1.19E-06 & 1.63E-08 & 8.77E-07 & $2.69 \mathrm{E}-07$ & $2.28 \mathrm{E}-08$ & $2.85 \mathrm{E}-07$ \\
\hline $\mathrm{Br}-82$ & 1.97E-04 & $6.35 \mathrm{E}-07$ & $1.95 \mathrm{E}-04$ & $1.15 \mathrm{E}-06$ & 4.90E-07 & $1.79 \mathrm{E}-06$ \\
\hline $\mathrm{Br}-83$ & 4.02E-08 & $1.31 \mathrm{E}-09$ & $3.89 \mathrm{E}-08$ & $0.00 \mathrm{E}+00$ & $0.00 \mathrm{E}+00$ & $1.31 \mathrm{E}-09$ \\
\hline $\mathrm{Br}-84$ & 8.86E-07 & $1.20 \mathrm{E}-07$ & $7.66 \mathrm{E}-07$ & $0.00 \mathrm{E}+00$ & $0.00 \mathrm{E}+00$ & $1.20 \mathrm{E}-07$ \\
\hline $\mathrm{Kr}-79$ & 7.37E-08 & 7.37E-08 & $0.00 \mathrm{E}+00$ & $0.00 \mathrm{E}+00$ & $0.00 \mathrm{E}+00$ & 7.37E-08 \\
\hline $\mathrm{Kr}-81$ & 3.02E-09 & 3.02E-09 & $0.00 \mathrm{E}+00$ & $0.00 \mathrm{E}+00$ & $0.00 \mathrm{E}+00$ & 3.02E-09 \\
\hline $\mathrm{Kr}-83 \mathrm{~m}$ & $1.85 \mathrm{E}-11$ & $1.85 \mathrm{E}-11$ & $0.00 \mathrm{E}+00$ & $0.00 \mathrm{E}+00$ & $0.00 \mathrm{E}+00$ & $1.85 \mathrm{E}-11$ \\
\hline $\mathrm{Kr}-85$ & $6.63 \mathrm{E}-10$ & $6.63 \mathrm{E}-10$ & $0.00 \mathrm{E}+00$ & $0.00 \mathrm{E}+00$ & $0.00 \mathrm{E}+00$ & $6.63 \mathrm{E}-10$ \\
\hline $\mathrm{Kr}-85 \mathrm{~m}$ & 4.08E-08 & $4.08 \mathrm{E}-08$ & $0.00 \mathrm{E}+00$ & $0.00 \mathrm{E}+00$ & $0.00 \mathrm{E}+00$ & $4.08 \mathrm{E}-08$ \\
\hline $\mathrm{Kr}-87$ & 1.47E-07 & $1.47 \mathrm{E}-07$ & $0.00 \mathrm{E}+00$ & $3.72 \mathrm{E}-21$ & 1.75E-22 & 1.47E-07 \\
\hline $\mathrm{Kr}-88$ & $8.21 \mathrm{E}-07$ & $6.76 \mathrm{E}-07$ & 3.94E-08 & 1.05E-07 & $3.21 \mathrm{E}-11$ & $7.81 \mathrm{E}-07$ \\
\hline $\mathrm{Rb}-81$ & 5.23E-07 & $1.52 \mathrm{E}-07$ & $3.71 \mathrm{E}-07$ & $0.00 \mathrm{E}+00$ & $0.00 \mathrm{E}+00$ & $1.52 \mathrm{E}-07$ \\
\hline $\mathrm{Rb}-82$ & $5.20 \mathrm{E}-22$ & $4.45 \mathrm{E}-22$ & $7.46 \mathrm{E}-23$ & $0.00 \mathrm{E}+00$ & $0.00 \mathrm{E}+00$ & $4.45 \mathrm{E}-22$ \\
\hline $\mathrm{Rb}-83$ & $8.24 \mathrm{E}-06$ & $1.46 \mathrm{E}-07$ & 8.09E-06 & $0.00 \mathrm{E}+00$ & $0.00 \mathrm{E}+00$ & 1.46E-07 \\
\hline
\end{tabular}


Table 2. Ground release, Class D, $4 \mathrm{~m} / \mathrm{s}$ wind, $1 \mathrm{~km}$, no rain, continued.

\begin{tabular}{|c|c|c|c|c|c|c|}
\hline Nuclide & $\begin{array}{c}\text { 7-day } \\
\text { Total } \\
(1+2+3+4)\end{array}$ & $\begin{array}{l}\text { Cloud } \\
\text { shine } \\
(1)\end{array}$ & $\begin{array}{c}\text { Ground } \\
\text { shine } \\
(2)\end{array}$ & $\begin{array}{c}\text { Inhalation } \\
(3)\end{array}$ & $\begin{array}{l}\text { Resus- } \\
\text { pension } \\
(4)\end{array}$ & $\begin{array}{c}\text { Plume } \\
\text { Passage } \\
(1)+(3)\end{array}$ \\
\hline $\mathrm{Rb}-84$ & 1.95E-05 & $2.71 \mathrm{E}-07$ & $1.35 \mathrm{E}-05$ & $5.49 \mathrm{E}-06$ & 2.47E-07 & $5.76 \mathrm{E}-06$ \\
\hline $\mathrm{Rb}-86$ & $9.41 \mathrm{E}-06$ & $2.88 \mathrm{E}-08$ & $1.27 \mathrm{E}-06$ & $7.78 \mathrm{E}-06$ & $3.38 \mathrm{E}-07$ & 7.81E-06 \\
\hline $\mathrm{Rb}-87$ & $2.85 \mathrm{E}-06$ & $0.00 \mathrm{E}+00$ & $0.00 \mathrm{E}+00$ & $2.73 \mathrm{E}-06$ & 1.29E-07 & $2.73 \mathrm{E}-06$ \\
\hline $\mathrm{Rb}-88$ & $3.26 \mathrm{E}-08$ & $1.72 \mathrm{E}-08$ & $4.21 \mathrm{E}-09$ & $1.12 \mathrm{E}-08$ & $3.44 \mathrm{E}-12$ & $2.84 \mathrm{E}-08$ \\
\hline $\mathrm{Rb}-89$ & $4.51 \mathrm{E}-08$ & $3.55 \mathrm{E}-08$ & 8.43E-09 & 1.12E-09 & $5.41 \mathrm{E}-11$ & $3.66 \mathrm{E}-08$ \\
\hline Sr-82 & $1.68 \mathrm{E}-05$ & $3.21 \mathrm{E}-07$ & $1.64 \mathrm{E}-05$ & $0.00 \mathrm{E}+00$ & $0.00 \mathrm{E}+00$ & $3.21 \mathrm{E}-07$ \\
\hline Sr-85 & 1.06E-05 & 1.49E-07 & $8.20 \mathrm{E}-06$ & $2.14 \mathrm{E}-06$ & $9.87 \mathrm{E}-08$ & $2.29 \mathrm{E}-06$ \\
\hline Sr-85m & 6.61E-08 & 3.37E-08 & $3.16 \mathrm{E}-08$ & $6.63 \mathrm{E}-10$ & 6.37E-11 & $3.44 \mathrm{E}-08$ \\
\hline Sr-87m & $1.85 \mathrm{E}-07$ & $7.15 \mathrm{E}-08$ & $1.14 \mathrm{E}-07$ & $1.29 \mathrm{E}-23$ & $2.51 \mathrm{E}-24$ & $7.15 \mathrm{E}-08$ \\
\hline Sr-89 & 5.93E-06 & $4.18 \mathrm{E}-11$ & $2.07 \mathrm{E}-09$ & 5.67E-06 & $2.59 \mathrm{E}-07$ & $5.67 \mathrm{E}-06$ \\
\hline Sr-90 & $4.50 \mathrm{E}-04$ & $0.00 \mathrm{E}+00$ & $0.00 \mathrm{E}+00$ & $4.30 \mathrm{E}-04$ & $2.05 \mathrm{E}-05$ & $4.30 \mathrm{E}-04$ \\
\hline Sr-91 & $2.46 \mathrm{E}-06$ & $2.44 \mathrm{E}-07$ & $1.26 \mathrm{E}-06$ & $9.25 \mathrm{E}-07$ & $2.17 \mathrm{E}-08$ & 1.17E-06 \\
\hline Sr-92 & $1.02 \mathrm{E}-06$ & $3.20 \mathrm{E}-07$ & $4.74 \mathrm{E}-07$ & $2.25 \mathrm{E}-07$ & 3.37E-09 & $5.45 \mathrm{E}-07$ \\
\hline$Y-86$ & 7.86E-06 & 1.06E-06 & $6.80 \mathrm{E}-06$ & $0.00 \mathrm{E}+00$ & $0.00 \mathrm{E}+00$ & 1.06E-06 \\
\hline Y-87 & 7.04E-06 & $1.54 \mathrm{E}-07$ & $6.89 \mathrm{E}-06$ & $1.78 \mathrm{E}-24$ & $2.38 \mathrm{E}-23$ & $1.54 \mathrm{E}-07$ \\
\hline$Y-88$ & 7.26E-05 & 8.66E-07 & $3.90 \mathrm{E}-05$ & $3.12 \mathrm{E}-05$ & $1.45 \mathrm{E}-06$ & $3.21 \mathrm{E}-05$ \\
\hline Y-90 & $1.02 \mathrm{E}-05$ & $0.00 \mathrm{E}+00$ & $0.00 \mathrm{E}+00$ & $9.95 \mathrm{E}-06$ & 2.87E-07 & $9.95 \mathrm{E}-06$ \\
\hline Y-90m & 9.83E-07 & $1.48 \mathrm{E}-07$ & $2.61 \mathrm{E}-07$ & 5.59E-07 & $1.51 \mathrm{E}-08$ & 7.07E-07 \\
\hline Y-91 & 5.72E-05 & 1.09E-09 & $5.10 \mathrm{E}-08$ & $5.46 \mathrm{E}-05$ & $2.51 \mathrm{E}-06$ & $5.46 \mathrm{E}-05$ \\
\hline Y-91m & $1.40 \mathrm{E}-07$ & $6.39 \mathrm{E}-08$ & $3.63 \mathrm{E}-08$ & $3.86 \mathrm{E}-08$ & 1.49E-09 & $1.03 \mathrm{E}-07$ \\
\hline Y-92 & $1.28 \mathrm{E}-06$ & $6.22 \mathrm{E}-08$ & $1.05 \mathrm{E}-07$ & $1.11 \mathrm{E}-06$ & $3.81 \mathrm{E}-09$ & 1.17E-06 \\
\hline Y-93 & $3.10 \mathrm{E}-06$ & $2.61 \mathrm{E}-08$ & $1.15 \mathrm{E}-07$ & $2.93 \mathrm{E}-06$ & $2.56 \mathrm{E}-08$ & $2.96 \mathrm{E}-06$ \\
\hline Y-94 & $1.53 \mathrm{E}-08$ & $0.00 \mathrm{E}+00$ & $0.00 \mathrm{E}+00$ & $1.53 \mathrm{E}-08$ & $5.01 \mathrm{E}-12$ & $1.53 \mathrm{E}-08$ \\
\hline $\mathrm{Zr}-86$ & 8.60E-06 & $1.31 \mathrm{E}-07$ & 8.47E-06 & $0.00 \mathrm{E}+00$ & $0.00 \mathrm{E}+00$ & 1.31E-07 \\
\hline $\mathrm{Zr}-88$ & 7.42E-06 & 1.12E-07 & $7.28 \mathrm{E}-06$ & $9.04 \mathrm{E}-09$ & $2.14 \mathrm{E}-08$ & $1.21 \mathrm{E}-07$ \\
\hline $\mathrm{Zr}-89$ & $1.26 \mathrm{E}-05$ & $3.47 \mathrm{E}-07$ & $9.60 \mathrm{E}-06$ & $2.55 \mathrm{E}-06$ & 7.94E-08 & $2.90 \mathrm{E}-06$ \\
\hline Zr-93 & 9.74E-05 & $2.46 \mathrm{E}-16$ & $8.15 \mathrm{E}-12$ & $9.30 \mathrm{E}-05$ & $4.38 \mathrm{E}-06$ & $9.30 \mathrm{E}-05$ \\
\hline Zr-95 & $3.12 \mathrm{E}-05$ & $2.23 \mathrm{E}-07$ & $1.24 \mathrm{E}-05$ & 1.77E-05 & 8.30E-07 & $1.79 \mathrm{E}-05$ \\
\hline Zr-97 & 9.01E-06 & $3.40 \mathrm{E}-07$ & $3.58 \mathrm{E}-06$ & $5.02 \mathrm{E}-06$ & $6.72 \mathrm{E}-08$ & 5.36E-06 \\
\hline $\mathrm{Nb}-90$ & 1.17E-05 & $1.30 \mathrm{E}-06$ & $7.54 \mathrm{E}-06$ & $2.81 \mathrm{E}-06$ & $3.31 \mathrm{E}-08$ & $4.11 \mathrm{E}-06$ \\
\hline $\mathrm{Nb}-92 \mathrm{~m}$ & $1.50 \mathrm{E}-05$ & $2.94 \mathrm{E}-07$ & $1.21 \mathrm{E}-05$ & $2.45 \mathrm{E}-06$ & 9.97E-08 & $2.74 \mathrm{E}-06$ \\
\hline $\mathrm{Nb}-93 \mathrm{~m}$ & 3.53E-05 & 3.96E-11 & $1.66 \mathrm{E}-08$ & 3.37E-05 & $1.59 \mathrm{E}-06$ & 3.37E-05 \\
\hline $\mathrm{Nb}-94 \mathrm{~m}$ & $3.00 \mathrm{E}-12$ & $1.16 \mathrm{E}-12$ & $4.27 \mathrm{E}-13$ & $1.40 \mathrm{E}-12$ & $1.18 \mathrm{E}-14$ & $2.56 \mathrm{E}-12$ \\
\hline $\mathrm{Nb}-94$ & 4.71E-04 & $4.78 \mathrm{E}-07$ & 2.54E-05 & $4.25 \mathrm{E}-04$ & $2.01 \mathrm{E}-05$ & $4.25 \mathrm{E}-04$ \\
\hline $\mathrm{Nb}-95$ & 1.86E-05 & $2.32 \mathrm{E}-07$ & $1.16 \mathrm{E}-05$ & $6.49 \mathrm{E}-06$ & 2.93E-07 & $6.72 \mathrm{E}-06$ \\
\hline $\mathrm{Nb}-95 \mathrm{~m}$ & 4.23E-06 & $1.77 \mathrm{E}-08$ & $1.16 \mathrm{E}-06$ & $2.95 \mathrm{E}-06$ & $1.05 \mathrm{E}-07$ & 2.97E-06 \\
\hline $\mathrm{Nb}-96$ & 1.13E-05 & $7.21 \mathrm{E}-07$ & 7.71E-06 & $2.78 \mathrm{E}-06$ & 4.61E-08 & $3.50 \mathrm{E}-06$ \\
\hline $\mathrm{Nb}-97$ & $2.75 \mathrm{E}-07$ & 1.07E-07 & 7.84E-08 & 8.93E-08 & $1.09 \mathrm{E}-10$ & 1.96E-07 \\
\hline $\mathrm{Nb}-97 \mathrm{~m}$ & 3.87E-09 & $1.51 \mathrm{E}-09$ & 1.10E-09 & 1.26E-09 & $1.53 \mathrm{E}-12$ & $2.77 \mathrm{E}-09$ \\
\hline $\mathrm{Nb}-98$ & $9.58 \mathrm{E}-08$ & $0.00 \mathrm{E}+00$ & $0.00 \mathrm{E}+00$ & $9.58 \mathrm{E}-08$ & $8.39 \mathrm{E}-11$ & $9.58 \mathrm{E}-08$ \\
\hline Mo-93 & $4.10 \mathrm{E}-05$ & $2.21 \mathrm{E}-10$ & $9.31 \mathrm{E}-08$ & 3.91E-05 & 1.84E-06 & 3.91E-05 \\
\hline
\end{tabular}


Table 2. Ground release, Class D, $4 \mathrm{~m} / \mathrm{s}$ wind, $1 \mathrm{~km}$, no rain, continued.

\begin{tabular}{|c|c|c|c|c|c|c|}
\hline Nuclide & $\begin{array}{c}\text { 7-day } \\
\text { Total } \\
(1+2+3+4)\end{array}$ & $\begin{array}{c}\text { Cloud } \\
\text { shine } \\
(1)\end{array}$ & $\begin{array}{l}\text { Ground } \\
\text { shine } \\
(2)\end{array}$ & $\begin{array}{c}\text { Inhalation } \\
\text { (3) }\end{array}$ & $\begin{array}{c}\text { Resus- } \\
\text { pension } \\
(4)\end{array}$ & $\begin{array}{c}\text { Plume } \\
\text { Passage } \\
(1)+(3)\end{array}$ \\
\hline Mo-93m & 5.43E-07 & $5.07 \mathrm{E}-18$ & 1.96E-14 & $5.40 \mathrm{E}-07$ & $3.38 \mathrm{E}-09$ & $5.40 \mathrm{E}-07$ \\
\hline Mo-99 & $6.98 \mathrm{E}-06$ & $5.00 \mathrm{E}-08$ & $2.15 \mathrm{E}-06$ & $4.64 \mathrm{E}-06$ & $1.36 \mathrm{E}-07$ & $4.69 \mathrm{E}-06$ \\
\hline Mo-101 & $5.45 \mathrm{E}-08$ & $3.65 \mathrm{E}-08$ & 1.04E-08 & 7.62E-09 & $2.25 \mathrm{E}-12$ & $4.41 \mathrm{E}-08$ \\
\hline Tc-95 & $2.71 \mathrm{E}-06$ & $2.30 \mathrm{E}-07$ & $2.16 \mathrm{E}-06$ & $3.14 \mathrm{E}-07$ & 4.67E-09 & $5.44 \mathrm{E}-07$ \\
\hline Tc-95m & 1.13E-05 & $2.00 \mathrm{E}-07$ & $1.11 \mathrm{E}-05$ & $4.74 \mathrm{E}-10$ & $4.17 \mathrm{E}-10$ & $2.00 \mathrm{E}-07$ \\
\hline Tc-96 & $2.77 \mathrm{E}-05$ & 7.56E-07 & $2.40 \mathrm{E}-05$ & 2.87E-06 & 9.77E-08 & $3.63 \mathrm{E}-06$ \\
\hline Tc-96m & $2.23 \mathrm{E}-07$ & 8.92E-09 & $2.00 \mathrm{E}-07$ & 1.36E-08 & $7.98 \mathrm{E}-10$ & $2.25 \mathrm{E}-08$ \\
\hline Tc-97 & $1.24 \mathrm{E}-06$ & $2.75 \mathrm{E}-10$ & $1.03 \mathrm{E}-07$ & $1.08 \mathrm{E}-06$ & 5.11E-08 & $1.08 \mathrm{E}-06$ \\
\hline Tc-97m & $8.72 \mathrm{E}-08$ & $3.38 \mathrm{E}-10$ & $8.69 \mathrm{E}-08$ & $3.52 \mathrm{E}-17$ & $8.44 \mathrm{E}-17$ & $3.38 \mathrm{E}-10$ \\
\hline Tc-98 & 4.66E-05 & $4.20 \mathrm{E}-07$ & $2.29 \mathrm{E}-05$ & $2.22 \mathrm{E}-05$ & 1.05E-06 & $2.26 \mathrm{E}-05$ \\
\hline Tc-99 & $8.01 \mathrm{E}-06$ & $1.53 \mathrm{E}-13$ & $9.97 \mathrm{E}-12$ & 7.65E-06 & $3.61 \mathrm{E}-07$ & $7.65 \mathrm{E}-06$ \\
\hline Tc-99m & $1.81 \mathrm{E}-07$ & $3.41 \mathrm{E}-08$ & $1.14 \mathrm{E}-07$ & $3.27 \mathrm{E}-08$ & $1.83 \mathrm{E}-10$ & $6.68 \mathrm{E}-08$ \\
\hline Tc-101 & $7.01 \mathrm{E}-09$ & 4.39E-09 & $1.29 \mathrm{E}-09$ & 1.32E-09 & $3.22 \mathrm{E}-13$ & 5.71E-09 \\
\hline Ru-97 & $2.60 \mathrm{E}-06$ & $6.72 \mathrm{E}-08$ & $2.01 \mathrm{E}-06$ & $5.10 \mathrm{E}-07$ & $1.52 \mathrm{E}-08$ & 5.77E-07 \\
\hline Ru-103 & $1.82 \mathrm{E}-05$ & 1.42E-07 & 7.57E-06 & $1.00 \mathrm{E}-05$ & $4.54 \mathrm{E}-07$ & $1.01 \mathrm{E}-05$ \\
\hline Ru-105 & $1.30 \mathrm{E}-06$ & 1.99E-07 & $5.00 \mathrm{E}-07$ & 5.95E-07 & $5.38 \mathrm{E}-09$ & 7.94E-07 \\
\hline Ru-106 & $5.60 \mathrm{E}-04$ & $6.15 \mathrm{E}-08$ & $3.36 \mathrm{E}-06$ & $5.32 \mathrm{E}-04$ & $2.50 \mathrm{E}-05$ & $5.32 \mathrm{E}-04$ \\
\hline Rh-101 & $4.38 \mathrm{E}-05$ & $0.00 \mathrm{E}+00$ & $0.00 \mathrm{E}+00$ & $4.18 \mathrm{E}-05$ & 1.97E-06 & $4.18 \mathrm{E}-05$ \\
\hline $\mathrm{Rh}-101 \mathrm{~m}$ & 8.68E-07 & $0.00 \mathrm{E}+00$ & $0.00 \mathrm{E}+00$ & 8.40E-07 & $2.88 \mathrm{E}-08$ & $8.40 \mathrm{E}-07$ \\
\hline Rh-102 & 1.37E-04 & $0.00 \mathrm{E}+00$ & $0.00 \mathrm{E}+00$ & $1.31 \mathrm{E}-04$ & $6.18 \mathrm{E}-06$ & $1.31 \mathrm{E}-04$ \\
\hline $\mathrm{Rh}-102 \mathrm{~m}$ & $2.57 \mathrm{E}-05$ & $0.00 \mathrm{E}+00$ & $0.00 \mathrm{E}+00$ & $2.45 \mathrm{E}-05$ & $1.15 \mathrm{E}-06$ & $2.45 \mathrm{E}-05$ \\
\hline $\mathrm{Rh}-103 \mathrm{~m}$ & 4.37E-09 & $2.65 \mathrm{E}-11$ & $9.26 \mathrm{E}-11$ & 4.24E-09 & $4.05 \mathrm{E}-12$ & 4.27E-09 \\
\hline Rh-105 & $1.59 \mathrm{E}-06$ & $2.23 \mathrm{E}-08$ & $3.90 \mathrm{E}-07$ & $1.15 \mathrm{E}-06$ & $2.47 \mathrm{E}-08$ & 1.17E-06 \\
\hline Rh-106 & $0.00 \mathrm{E}+00$ & $0.00 \mathrm{E}+00$ & $0.00 \mathrm{E}+00$ & $0.00 \mathrm{E}+00$ & $0.00 \mathrm{E}+00$ & $0.00 \mathrm{E}+00$ \\
\hline $\mathrm{Pd}-103$ & $1.65 \mathrm{E}-07$ & $5.52 \mathrm{E}-10$ & $1.59 \mathrm{E}-07$ & $5.13 \mathrm{E}-09$ & $4.00 \mathrm{E}-10$ & $5.68 \mathrm{E}-09$ \\
\hline Pd-107 & $1.50 \mathrm{E}-05$ & $0.00 \mathrm{E}+00$ & $0.00 \mathrm{E}+00$ & 1.43E-05 & $6.75 \mathrm{E}-07$ & $1.43 \mathrm{E}-05$ \\
\hline Pd-109 & $1.46 \mathrm{E}-06$ & $1.91 \mathrm{E}-10$ & $1.28 \mathrm{E}-09$ & $1.45 \mathrm{E}-06$ & 1.59E-08 & $1.45 \mathrm{E}-06$ \\
\hline $\mathrm{Ag}-106$ & 8.43E-09 & $0.00 \mathrm{E}+00$ & $0.00 \mathrm{E}+00$ & 8.43E-09 & $3.47 \mathrm{E}-12$ & 8.43E-09 \\
\hline $\mathrm{Ag}-106 \mathrm{~m}$ & 4.09E-05 & $8.40 \mathrm{E}-07$ & $3.35 \mathrm{E}-05$ & $6.34 \mathrm{E}-06$ & $2.51 \mathrm{E}-07$ & $7.18 \mathrm{E}-06$ \\
\hline $\mathrm{Ag}-108$ & $8.79 \mathrm{E}-17$ & $3.67 \mathrm{E}-17$ & $6.16 \mathrm{E}-18$ & $4.51 \mathrm{E}-17$ & $0.00 \mathrm{E}+00$ & $8.18 \mathrm{E}-17$ \\
\hline $\mathrm{Ag}-108 \mathrm{~m}$ & $3.43 \mathrm{E}-04$ & $4.80 \mathrm{E}-07$ & $2.68 \mathrm{E}-05$ & 3.01E-04 & 1.42E-05 & $3.01 \mathrm{E}-04$ \\
\hline $\mathrm{Ag}-109 \mathrm{~m}$ & $0.00 \mathrm{E}+00$ & $0.00 \mathrm{E}+00$ & $0.00 \mathrm{E}+00$ & $0.00 \mathrm{E}+00$ & $0.00 \mathrm{E}+00$ & $0.00 \mathrm{E}+00$ \\
\hline $\mathrm{Ag}-110$ & $0.00 \mathrm{E}+00$ & $0.00 \mathrm{E}+00$ & $0.00 \mathrm{E}+00$ & $0.00 \mathrm{E}+00$ & $0.00 \mathrm{E}+00$ & $0.00 \mathrm{E}+00$ \\
\hline $\mathrm{Ag}-110 \mathrm{~m}$ & $1.39 \mathrm{E}-04$ & $8.32 \mathrm{E}-07$ & 4.26E-05 & $9.09 \mathrm{E}-05$ & $4.26 \mathrm{E}-06$ & 9.17E-05 \\
\hline $\mathrm{Ag}-111$ & $7.82 \mathrm{E}-06$ & 7.70E-09 & 3.34E-07 & 7.20E-06 & $2.79 \mathrm{E}-07$ & $7.21 \mathrm{E}-06$ \\
\hline Cd-109 & $3.94 \mathrm{E}-05$ & $6.44 \mathrm{E}-10$ & $1.51 \mathrm{E}-07$ & $3.75 \mathrm{E}-05$ & $1.76 \mathrm{E}-06$ & $3.75 \mathrm{E}-05$ \\
\hline Cd-113 & $0.00 \mathrm{E}+00$ & $0.00 \mathrm{E}+00$ & $0.00 \mathrm{E}+00$ & $0.00 \mathrm{E}+00$ & $0.00 \mathrm{E}+00$ & $0.00 \mathrm{E}+00$ \\
\hline Cd-113m & 4.65E-04 & $0.00 \mathrm{E}+00$ & $0.00 \mathrm{E}+00$ & $4.44 \mathrm{E}-04$ & $2.09 \mathrm{E}-05$ & 4.44E-04 \\
\hline Cd-115 & $7.98 \mathrm{E}-06$ & $6.60 \mathrm{E}-08$ & $2.49 \mathrm{E}-06$ & $5.28 \mathrm{E}-06$ & $1.45 \mathrm{E}-07$ & $5.35 \mathrm{E}-06$ \\
\hline Cd-115m & $3.25 \mathrm{E}-07$ & 6.67E-09 & 3.19E-07 & $1.89 \mathrm{E}-23$ & $4.48 \mathrm{E}-23$ & 6.67E-09 \\
\hline $\mathrm{Cd}-117$ & $7.28 \mathrm{E}-07$ & $2.71 \mathrm{E}-07$ & 4.57E-07 & $0.00 \mathrm{E}+00$ & $0.00 \mathrm{E}+00$ & $2.71 \mathrm{E}-07$ \\
\hline
\end{tabular}


Table 2. Ground release, Class D, $4 \mathrm{~m} / \mathrm{s}$ wind, $1 \mathrm{~km}$, no rain, continued.

\begin{tabular}{|c|c|c|c|c|c|c|}
\hline Nuclide & $\begin{array}{c}\text { 7-day } \\
\text { Total } \\
(1+2+3+4)\end{array}$ & $\begin{array}{c}\text { Cloud } \\
\text { shine } \\
(1)\end{array}$ & $\begin{array}{l}\text { Ground } \\
\text { shine } \\
(2)\end{array}$ & $\begin{array}{c}\text { Inhalation } \\
\text { (3) }\end{array}$ & $\begin{array}{l}\text { Resus- } \\
\text { pension } \\
(4)\end{array}$ & $\begin{array}{c}\text { Plume } \\
\text { Passage } \\
(1)+(3)\end{array}$ \\
\hline Cd-117m & 1.74E-06 & $6.40 \mathrm{E}-07$ & $1.11 \mathrm{E}-06$ & $0.00 \mathrm{E}+00$ & $0.00 \mathrm{E}+00$ & $6.40 \mathrm{E}-07$ \\
\hline $\ln -111$ & 3.49E-06 & $1.15 \mathrm{E}-07$ & $3.37 \mathrm{E}-06$ & $0.00 \mathrm{E}+00$ & $0.00 \mathrm{E}+00$ & 1.15E-07 \\
\hline In-113m & 9.59E-08 & $4.76 \mathrm{E}-08$ & $4.84 \mathrm{E}-08$ & $0.00 \mathrm{E}+00$ & $0.00 \mathrm{E}+00$ & $4.76 \mathrm{E}-08$ \\
\hline $\ln -114$ & $0.00 \mathrm{E}+00$ & $0.00 \mathrm{E}+00$ & $0.00 \mathrm{E}+00$ & $0.00 \mathrm{E}+00$ & $0.00 \mathrm{E}+00$ & $0.00 \mathrm{E}+00$ \\
\hline In-114m & 6.79E-05 & $3.58 \mathrm{E}-08$ & 1.97E-06 & $6.30 \mathrm{E}-05$ & $2.88 \mathrm{E}-06$ & $6.30 \mathrm{E}-05$ \\
\hline $\ln -115$ & 1.20E-03 & $0.00 \mathrm{E}+00$ & $0.00 \mathrm{E}+00$ & 1.14E-03 & 5.39E-05 & $1.14 \mathrm{E}-03$ \\
\hline In-115m & $3.25 \mathrm{E}-07$ & $3.95 \mathrm{E}-08$ & 9.83E-08 & $1.87 \mathrm{E}-07$ & $8.00 \mathrm{E}-10$ & $2.27 \mathrm{E}-07$ \\
\hline In-116m & 5.53E-07 & $3.32 \mathrm{E}-07$ & 1.71E-07 & $4.90 \mathrm{E}-08$ & $4.51 \mathrm{E}-11$ & $3.81 \mathrm{E}-07$ \\
\hline $\ln -117$ & 1.13E-07 & $7.38 \mathrm{E}-08$ & $3.92 \mathrm{E}-08$ & $0.00 \mathrm{E}+00$ & $0.00 \mathrm{E}+00$ & $7.38 \mathrm{E}-08$ \\
\hline In-117m & 1.77E-07 & $6.71 \mathrm{E}-08$ & 1.10E-07 & $0.00 \mathrm{E}+00$ & $0.00 \mathrm{E}+00$ & $6.71 \mathrm{E}-08$ \\
\hline Sn-113 & 1.49E-05 & 2.93E-08 & $4.48 \mathrm{E}-06$ & 9.96E-06 & 4.63E-07 & $9.99 \mathrm{E}-06$ \\
\hline Sn-117m & 2.34E-06 & $4.38 \mathrm{E}-08$ & $2.30 \mathrm{E}-06$ & $0.00 \mathrm{E}+00$ & $0.00 \mathrm{E}+00$ & $4.38 \mathrm{E}-08$ \\
\hline Sn-119m & 7.13E-06 & $6.38 \mathrm{E}-10$ & $9.55 \mathrm{E}-08$ & $6.72 \mathrm{E}-06$ & $3.15 \mathrm{E}-07$ & $6.72 \mathrm{E}-06$ \\
\hline Sn-121 & $6.41 \mathrm{E}-07$ & $0.00 \mathrm{E}+00$ & $0.00 \mathrm{E}+00$ & $6.30 \mathrm{E}-07$ & $1.15 \mathrm{E}-08$ & $6.30 \mathrm{E}-07$ \\
\hline Sn-121m & 1.96E-05 & $0.00 \mathrm{E}+00$ & $0.00 \mathrm{E}+00$ & 1.87E-05 & 8.98E-07 & 1.87E-05 \\
\hline Sn-123 & $3.83 \mathrm{E}-05$ & $2.10 \mathrm{E}-09$ & 1.03E-07 & $3.65 \mathrm{E}-05$ & 1.70E-06 & 3.65E-05 \\
\hline Sn-125 & 3.76E-06 & $9.40 \mathrm{E}-08$ & 3.67E-06 & $3.94 \mathrm{E}-10$ & 8.47E-10 & 9.44E-08 \\
\hline Sn-126 & $1.55 \mathrm{E}-04$ & 4.39E-07 & $2.80 \mathrm{E}-05$ & $1.21 \mathrm{E}-04$ & 5.73E-06 & $1.21 \mathrm{E}-04$ \\
\hline Sb-117 & $1.05 \mathrm{E}-07$ & $3.92 \mathrm{E}-08$ & $6.56 \mathrm{E}-08$ & $0.00 \mathrm{E}+00$ & $0.00 \mathrm{E}+00$ & $3.92 \mathrm{E}-08$ \\
\hline Sb-120b & $4.85 \mathrm{E}-06$ & $0.00 \mathrm{E}+00$ & $0.00 \mathrm{E}+00$ & $4.68 \mathrm{E}-06$ & $1.72 \mathrm{E}-07$ & $4.68 \mathrm{E}-06$ \\
\hline Sb-122 & $9.68 \mathrm{E}-06$ & $1.30 \mathrm{E}-07$ & $3.37 \mathrm{E}-06$ & $6.01 \mathrm{E}-06$ & $1.74 \mathrm{E}-07$ & $6.14 \mathrm{E}-06$ \\
\hline Sb-124 & 5.72E-05 & $5.79 \mathrm{E}-07$ & $2.72 \mathrm{E}-05$ & $2.81 \mathrm{E}-05$ & $1.29 \mathrm{E}-06$ & $2.87 \mathrm{E}-05$ \\
\hline Sb-125 & $2.08 \mathrm{E}-05$ & $1.25 \mathrm{E}-07$ & $7.10 \mathrm{E}-06$ & $1.29 \mathrm{E}-05$ & $6.11 \mathrm{E}-07$ & $1.30 \mathrm{E}-05$ \\
\hline Sb-126 & 5.36E-05 & $8.24 \mathrm{E}-07$ & 3.73E-05 & 1.49E-05 & $6.22 \mathrm{E}-07$ & $1.57 \mathrm{E}-05$ \\
\hline Sb-126m & 7.32E-08 & 4.53E-08 & $1.98 \mathrm{E}-08$ & 8.00E-09 & $9.47 \mathrm{E}-11$ & 5.33E-08 \\
\hline Sb-127 & $6.41 \mathrm{E}-06$ & $1.96 \mathrm{E}-07$ & $6.21 \mathrm{E}-06$ & $0.00 \mathrm{E}+00$ & $0.00 \mathrm{E}+00$ & $1.96 \mathrm{E}-07$ \\
\hline Sb-129 & $2.11 \mathrm{E}-06$ & $3.74 \mathrm{E}-07$ & 7.91E-07 & $9.38 \mathrm{E}-07$ & $5.48 \mathrm{E}-09$ & $1.31 \mathrm{E}-06$ \\
\hline Te-121 & 1.05E-05 & $1.65 \mathrm{E}-07$ & $8.15 \mathrm{E}-06$ & $2.11 \mathrm{E}-06$ & $9.09 \mathrm{E}-08$ & $2.28 \mathrm{E}-06$ \\
\hline Te-121m & 1.95E-05 & $6.17 \mathrm{E}-08$ & 4.69E-06 & 1.41E-05 & 6.64E-07 & $1.42 \mathrm{E}-05$ \\
\hline Te-123 & $5.40 \mathrm{E}-06$ & $8.84 \mathrm{E}-10$ & $1.14 \mathrm{E}-07$ & 5.04E-06 & $2.38 \mathrm{E}-07$ & 5.04E-06 \\
\hline Te-123m & 7.01E-06 & 4.19E-08 & $2.56 \mathrm{E}-06$ & 4.22E-06 & 1.96E-07 & $4.26 \mathrm{E}-06$ \\
\hline Te-125m & 8.69E-06 & $2.80 \mathrm{E}-09$ & $3.21 \mathrm{E}-07$ & $8.00 \mathrm{E}-06$ & 3.67E-07 & $8.00 \mathrm{E}-06$ \\
\hline Te-127 & 7.66E-09 & 1.31E-09 & $6.35 \mathrm{E}-09$ & $0.00 \mathrm{E}+00$ & $0.00 \mathrm{E}+00$ & 1.31E-09 \\
\hline Te-127m & $1.78 \mathrm{E}-07$ & $1.01 \mathrm{E}-09$ & $1.77 \mathrm{E}-07$ & $0.00 \mathrm{E}+00$ & $0.00 \mathrm{E}+00$ & $1.01 \mathrm{E}-09$ \\
\hline Te-129 & 9.56E-08 & 8.41E-09 & $6.25 \mathrm{E}-09$ & 8.09E-08 & $9.53 \mathrm{E}-11$ & 8.93E-08 \\
\hline Te-129m & 2.94E-05 & $1.50 \mathrm{E}-08$ & $1.10 \mathrm{E}-06$ & $2.71 \mathrm{E}-05$ & $1.22 \mathrm{E}-06$ & $2.71 \mathrm{E}-05$ \\
\hline Te-131 & $2.74 \mathrm{E}-07$ & $2.14 \mathrm{E}-08$ & $1.51 \mathrm{E}-07$ & $6.34 \mathrm{E}-08$ & $3.85 \mathrm{E}-08$ & $8.48 \mathrm{E}-08$ \\
\hline Te-131m & 1.10E-05 & 4.47E-07 & $6.90 \mathrm{E}-06$ & $3.44 \mathrm{E}-06$ & 2.57E-07 & $3.89 \mathrm{E}-06$ \\
\hline Te-132 & 3.36E-05 & 2.18E-07 & $2.45 \mathrm{E}-05$ & $8.59 \mathrm{E}-06$ & $2.80 \mathrm{E}-07$ & 8.81E-06 \\
\hline Te-133 & 3.84E-07 & $9.26 \mathrm{E}-09$ & $2.88 \mathrm{E}-07$ & $6.66 \mathrm{E}-08$ & $2.02 \mathrm{E}-08$ & $7.59 \mathrm{E}-08$ \\
\hline Te-133m & $1.48 \mathrm{E}-06$ & $3.28 \mathrm{E}-07$ & $9.30 \mathrm{E}-07$ & 1.65E-07 & $5.28 \mathrm{E}-08$ & 4.93E-07 \\
\hline
\end{tabular}


Table 2. Ground release, Class D, $4 \mathrm{~m} / \mathrm{s}$ wind, $1 \mathrm{~km}$, no rain, continued.

\begin{tabular}{|c|c|c|c|c|c|c|}
\hline Nuclide & $\begin{array}{c}\text { 7-day } \\
\text { Total } \\
(1+2+3+4)\end{array}$ & $\begin{array}{l}\text { Cloud } \\
\text { shine } \\
(1)\end{array}$ & $\begin{array}{c}\text { Ground } \\
\text { shine } \\
(2)\end{array}$ & $\begin{array}{c}\text { Inhalation } \\
(3)\end{array}$ & $\begin{array}{l}\text { Resus- } \\
\text { pension } \\
(4)\end{array}$ & $\begin{array}{c}\text { Plume } \\
\text { Passage } \\
(1)+(3)\end{array}$ \\
\hline Te-134 & $2.78 \mathrm{E}-06$ & 3.03E-07 & $2.44 \mathrm{E}-06$ & $4.20 \mathrm{E}-08$ & $7.85 \mathrm{E}-10$ & $3.45 \mathrm{E}-07$ \\
\hline $\mid-122$ & $5.25 \mathrm{E}-12$ & $1.07 \mathrm{E}-12$ & $4.18 \mathrm{E}-12$ & $0.00 \mathrm{E}+00$ & $0.00 \mathrm{E}+00$ & $1.07 \mathrm{E}-12$ \\
\hline $\mid-123$ & $5.28 \mathrm{E}-06$ & $3.61 \mathrm{E}-08$ & $5.25 \mathrm{E}-06$ & $5.27 \mathrm{E}-14$ & $5.33 \mathrm{E}-13$ & $3.61 \mathrm{E}-08$ \\
\hline $\mid-124$ & $1.58 \mathrm{E}-04$ & 2.59E-07 & $1.58 \mathrm{E}-04$ & $0.00 \mathrm{E}+00$ & $0.00 \mathrm{E}+00$ & $2.59 \mathrm{E}-07$ \\
\hline $\mid-125$ & 6.10E-06 & $2.63 \mathrm{E}-09$ & $6.09 \mathrm{E}-06$ & $0.00 \mathrm{E}+00$ & $0.00 \mathrm{E}+00$ & $2.63 \mathrm{E}-09$ \\
\hline $\mid-126$ & 1.32E-04 & 1.10E-07 & $1.02 \mathrm{E}-04$ & $1.59 \mathrm{E}-05$ & $1.32 \mathrm{E}-05$ & $1.60 \mathrm{E}-05$ \\
\hline $\mid-128$ & $3.45 \mathrm{E}-08$ & $3.02 \mathrm{E}-09$ & $2.26 \mathrm{E}-08$ & $8.88 \mathrm{E}-09$ & $7.56 \mathrm{E}-11$ & $1.19 \mathrm{E}-08$ \\
\hline $\mid-129$ & $2.70 \mathrm{E}-04$ & 1.96E-09 & $5.64 \mathrm{E}-06$ & $1.37 \mathrm{E}-04$ & $1.28 \mathrm{E}-04$ & 1.37E-04 \\
\hline $\mid-130$ & $6.11 \mathrm{E}-05$ & $4.89 \mathrm{E}-07$ & $5.82 \mathrm{E}-05$ & $2.08 \mathrm{E}-06$ & $4.25 \mathrm{E}-07$ & $2.57 \mathrm{E}-06$ \\
\hline $\mid-131$ & $1.27 \mathrm{E}-04$ & $9.07 \mathrm{E}-08$ & $7.85 \mathrm{E}-05$ & $2.71 \mathrm{E}-05$ & $2.11 \mathrm{E}-05$ & $2.72 \mathrm{E}-05$ \\
\hline $\mid-132$ & $1.04 \mathrm{E}-05$ & $4.08 \mathrm{E}-07$ & $9.79 \mathrm{E}-06$ & $2.32 \mathrm{E}-07$ & $1.05 \mathrm{E}-08$ & $6.40 \mathrm{E}-07$ \\
\hline $\mid-133$ & 3.82E-05 & $1.41 \mathrm{E}-07$ & $2.92 \mathrm{E}-05$ & $6.80 \mathrm{E}-06$ & 2.06E-06 & $6.94 \mathrm{E}-06$ \\
\hline $\mid-134$ & 3.36E-06 & $2.80 \mathrm{E}-07$ & $3.02 \mathrm{E}-06$ & $5.51 \mathrm{E}-08$ & $9.79 \mathrm{E}-10$ & $3.35 \mathrm{E}-07$ \\
\hline $\mid-135$ & $2.72 \mathrm{E}-05$ & $3.76 \mathrm{E}-07$ & $2.45 \mathrm{E}-05$ & 2.00E-06 & $2.41 \mathrm{E}-07$ & $2.38 \mathrm{E}-06$ \\
\hline Xe-122 & $1.16 \mathrm{E}-06$ & $2.51 \mathrm{E}-07$ & $9.09 \mathrm{E}-07$ & $0.00 \mathrm{E}+00$ & $0.00 \mathrm{E}+00$ & $2.51 \mathrm{E}-07$ \\
\hline Xe-123 & 3.89E-07 & $1.34 \mathrm{E}-07$ & $2.56 \mathrm{E}-07$ & $1.33 \mathrm{E}-15$ & $2.59 \mathrm{E}-14$ & $1.34 \mathrm{E}-07$ \\
\hline Xe-125 & $7.40 \mathrm{E}-08$ & $7.09 \mathrm{E}-08$ & $3.08 \mathrm{E}-09$ & $0.00 \mathrm{E}+00$ & $0.00 \mathrm{E}+00$ & $7.09 \mathrm{E}-08$ \\
\hline Xe-127 & 7.81E-08 & 7.81E-08 & $0.00 \mathrm{E}+00$ & $0.00 \mathrm{E}+00$ & $0.00 \mathrm{E}+00$ & 7.81E-08 \\
\hline $\mathrm{Xe}-129 \mathrm{~m}$ & 6.79E-09 & $6.79 \mathrm{E}-09$ & $0.00 \mathrm{E}+00$ & $0.00 \mathrm{E}+00$ & $0.00 \mathrm{E}+00$ & $6.79 \mathrm{E}-09$ \\
\hline $\mathrm{Xe}-131 \mathrm{~m}$ & 2.51E-09 & $2.51 \mathrm{E}-09$ & $0.00 \mathrm{E}+00$ & $0.00 \mathrm{E}+00$ & $0.00 \mathrm{E}+00$ & 2.51E-09 \\
\hline Xe-133 & 1.02E-08 & $1.02 \mathrm{E}-08$ & $0.00 \mathrm{E}+00$ & $0.00 \mathrm{E}+00$ & $0.00 \mathrm{E}+00$ & $1.02 \mathrm{E}-08$ \\
\hline Xe-133m & 8.70E-09 & 8.70E-09 & $0.00 \mathrm{E}+00$ & $0.00 \mathrm{E}+00$ & $0.00 \mathrm{E}+00$ & 8.70E-09 \\
\hline Xe-135 & $6.82 \mathrm{E}-08$ & $6.82 \mathrm{E}-08$ & $0.00 \mathrm{E}+00$ & $1.64 \mathrm{E}-16$ & $7.72 \mathrm{E}-18$ & $6.82 \mathrm{E}-08$ \\
\hline $\mathrm{Xe}-135 \mathrm{~m}$ & 8.77E-09 & 8.77E-09 & $0.00 \mathrm{E}+00$ & $3.13 \mathrm{E}-18$ & 1.47E-19 & 8.77E-09 \\
\hline Xe-138 & 1.80E-07 & $1.38 \mathrm{E}-07$ & $4.28 \mathrm{E}-08$ & $0.00 \mathrm{E}+00$ & $0.00 \mathrm{E}+00$ & $1.38 \mathrm{E}-07$ \\
\hline Cs-126 & $6.45 \mathrm{E}-19$ & $5.53 \mathrm{E}-19$ & $9.29 \mathrm{E}-20$ & $0.00 \mathrm{E}+00$ & $0.00 \mathrm{E}+00$ & $5.53 \mathrm{E}-19$ \\
\hline Cs-129 & 1.32E-06 & 7.44E-08 & $1.25 \mathrm{E}-06$ & $0.00 \mathrm{E}+00$ & $0.00 \mathrm{E}+00$ & $7.44 \mathrm{E}-08$ \\
\hline Cs-131 & $2.50 \mathrm{E}-07$ & 2.06E-09 & $2.48 \mathrm{E}-07$ & $0.00 \mathrm{E}+00$ & $0.00 \mathrm{E}+00$ & $2.06 \mathrm{E}-09$ \\
\hline Cs-132 & 9.77E-06 & $2.09 \mathrm{E}-07$ & $8.19 \mathrm{E}-06$ & $1.32 \mathrm{E}-06$ & 4.99E-08 & $1.53 \mathrm{E}-06$ \\
\hline Cs-134 & 8.06E-05 & 4.67E-07 & $2.52 \mathrm{E}-05$ & $5.25 \mathrm{E}-05$ & $2.47 \mathrm{E}-06$ & $5.30 \mathrm{E}-05$ \\
\hline Cs-134m & $2.09 \mathrm{E}-08$ & 4.69E-09 & 1.39E-08 & 1.90E-09 & $3.78 \mathrm{E}-10$ & $6.59 \mathrm{E}-09$ \\
\hline Cs-135 & 4.86E-06 & $0.00 \mathrm{E}+00$ & $0.00 \mathrm{E}+00$ & 4.64E-06 & $2.19 \mathrm{E}-07$ & $4.64 \mathrm{E}-06$ \\
\hline Cs-136 & 3.80E-05 & $6.54 \mathrm{E}-07$ & $2.88 \mathrm{E}-05$ & $8.22 \mathrm{E}-06$ & $3.45 \mathrm{E}-07$ & 8.87E-06 \\
\hline Cs-137 & 4.89E-05 & $1.69 \mathrm{E}-07$ & 9.27E-06 & $3.77 \mathrm{E}-05$ & $1.78 \mathrm{E}-06$ & 3.79E-05 \\
\hline Cs-138 & 2.53E-07 & 1.87E-07 & $6.59 \mathrm{E}-08$ & $0.00 \mathrm{E}+00$ & $0.00 \mathrm{E}+00$ & 1.87E-07 \\
\hline Ba-131 & $6.71 \mathrm{E}-06$ & $1.33 \mathrm{E}-07$ & 6.57E-06 & $0.00 \mathrm{E}+00$ & $0.00 \mathrm{E}+00$ & 1.33E-07 \\
\hline Ba-133 & 1.48E-05 & $1.07 \mathrm{E}-07$ & $6.71 \mathrm{E}-06$ & 7.66E-06 & 3.61E-07 & 7.77E-06 \\
\hline Ba-133m & 1.03E-06 & $1.62 \mathrm{E}-08$ & $3.53 \mathrm{E}-07$ & 6.47E-07 & $1.48 \mathrm{E}-08$ & $6.63 \mathrm{E}-07$ \\
\hline Ba-135m & $2.54 \mathrm{E}-07$ & $1.42 \mathrm{E}-08$ & $2.40 \mathrm{E}-07$ & $0.00 \mathrm{E}+00$ & $0.00 \mathrm{E}+00$ & $1.42 \mathrm{E}-08$ \\
\hline $\mathrm{Ba}-137 \mathrm{~m}$ & 5.61E-15 & $4.82 \mathrm{E}-15$ & $7.90 \mathrm{E}-16$ & $0.00 \mathrm{E}+00$ & $0.00 \mathrm{E}+00$ & $4.82 \mathrm{E}-15$ \\
\hline Ba-139 & 1.39E-07 & $6.12 \mathrm{E}-09$ & $5.39 \mathrm{E}-09$ & $1.27 \mathrm{E}-07$ & $1.78 \mathrm{E}-10$ & 1.33E-07 \\
\hline
\end{tabular}


Table 2. Ground release, Class D, $4 \mathrm{~m} / \mathrm{s}$ wind, $1 \mathrm{~km}$, no rain, continued.

\begin{tabular}{|c|c|c|c|c|c|c|}
\hline Nuclide & $\begin{array}{c}\text { 7-day } \\
\text { Total } \\
(1+2+3+4)\end{array}$ & $\begin{array}{l}\text { Cloud } \\
\text { shine } \\
(1)\end{array}$ & $\begin{array}{c}\text { Ground } \\
\text { shine } \\
(2)\end{array}$ & $\begin{array}{c}\text { Inhalation } \\
(3)\end{array}$ & $\begin{array}{l}\text { Resus- } \\
\text { pension } \\
(4)\end{array}$ & $\begin{array}{c}\text { Plume } \\
\text { Passage } \\
(1)+(3)\end{array}$ \\
\hline Ba-140 & 2.72E-05 & $6.79 \mathrm{E}-08$ & $2.26 \mathrm{E}-05$ & $4.23 \mathrm{E}-06$ & 3.03E-07 & $4.30 \mathrm{E}-06$ \\
\hline Ba-141 & $3.40 \mathrm{E}-08$ & $2.43 \mathrm{E}-08$ & 9.07E-09 & $4.31 \mathrm{E}-10$ & $1.64 \mathrm{E}-10$ & $2.47 \mathrm{E}-08$ \\
\hline Ba-142 & $1.27 \mathrm{E}-07$ & $7.54 \mathrm{E}-08$ & $5.15 \mathrm{E}-08$ & $0.00 \mathrm{E}+00$ & $0.00 \mathrm{E}+00$ & $7.54 \mathrm{E}-08$ \\
\hline La-137 & $2.58 \mathrm{E}-05$ & $0.00 \mathrm{E}+00$ & $0.00 \mathrm{E}+00$ & $2.47 \mathrm{E}-05$ & 1.16E-06 & $2.47 \mathrm{E}-05$ \\
\hline La-138 & 4.10E-04 & $0.00 \mathrm{E}+00$ & $0.00 \mathrm{E}+00$ & $3.91 \mathrm{E}-04$ & $1.84 \mathrm{E}-05$ & $3.91 \mathrm{E}-04$ \\
\hline La-140 & $1.77 \mathrm{E}-05$ & $7.01 \mathrm{E}-07$ & $1.11 \mathrm{E}-05$ & $5.85 \mathrm{E}-06$ & $1.35 \mathrm{E}-07$ & $6.55 \mathrm{E}-06$ \\
\hline La-141 & $4.65 \mathrm{E}-08$ & $1.07 \mathrm{E}-08$ & $2.49 \mathrm{E}-08$ & $8.72 \mathrm{E}-09$ & 2.13E-09 & $1.94 \mathrm{E}-08$ \\
\hline La-142 & $9.58 \mathrm{E}-07$ & $5.65 \mathrm{E}-07$ & 3.93E-07 & $0.00 \mathrm{E}+00$ & $0.00 \mathrm{E}+00$ & $5.65 \mathrm{E}-07$ \\
\hline Ce-139 & $1.27 \mathrm{E}-05$ & $4.36 \mathrm{E}-08$ & $2.77 \mathrm{E}-06$ & $9.46 \mathrm{E}-06$ & $4.41 \mathrm{E}-07$ & $9.50 \mathrm{E}-06$ \\
\hline $\mathrm{Ce}-141$ & $1.18 \mathrm{E}-05$ & $2.24 \mathrm{E}-08$ & $1.30 \mathrm{E}-06$ & $1.01 \mathrm{E}-05$ & $4.52 \mathrm{E}-07$ & $1.01 \mathrm{E}-05$ \\
\hline Ce-143 & $1.32 \mathrm{E}-06$ & $7.50 \mathrm{E}-08$ & $1.25 \mathrm{E}-06$ & $0.00 \mathrm{E}+00$ & $0.00 \mathrm{E}+00$ & $7.50 \mathrm{E}-08$ \\
\hline Ce-144 & 4.32E-04 & $1.49 \mathrm{E}-08$ & $8.18 \mathrm{E}-07$ & $4.12 \mathrm{E}-04$ & 1.93E-05 & $4.12 \mathrm{E}-04$ \\
\hline Pr-142 & 3.84E-06 & $1.74 \mathrm{E}-08$ & $1.32 \mathrm{E}-07$ & $3.64 \mathrm{E}-06$ & $5.24 \mathrm{E}-08$ & $3.66 \mathrm{E}-06$ \\
\hline $\mathrm{Pr}-143$ & $1.25 \mathrm{E}-13$ & $2.69 \mathrm{E}-15$ & $1.22 \mathrm{E}-13$ & $0.00 \mathrm{E}+00$ & $0.00 \mathrm{E}+00$ & $2.69 \mathrm{E}-15$ \\
\hline $\mathrm{Pr}-144$ & $9.94 \mathrm{E}-10$ & $7.95 \mathrm{E}-10$ & $1.99 \mathrm{E}-10$ & $0.00 \mathrm{E}+00$ & $0.00 \mathrm{E}+00$ & $7.95 \mathrm{E}-10$ \\
\hline Pr-144m & $6.96 \mathrm{E}-10$ & $5.55 \mathrm{E}-10$ & $1.41 \mathrm{E}-10$ & $0.00 \mathrm{E}+00$ & $0.00 \mathrm{E}+00$ & $5.55 \mathrm{E}-10$ \\
\hline $\mathrm{Nd}-141$ & $1.09 \mathrm{E}-08$ & $0.00 \mathrm{E}+00$ & $0.00 \mathrm{E}+00$ & 1.09E-08 & $2.69 \mathrm{E}-11$ & $1.09 \mathrm{E}-08$ \\
\hline $\mathrm{Nd}-147$ & 9.99E-06 & 3.87E-08 & $1.92 \mathrm{E}-06$ & 7.71E-06 & $3.20 \mathrm{E}-07$ & 7.75E-06 \\
\hline Nd-149 & $4.25 \mathrm{E}-07$ & $7.25 \mathrm{E}-08$ & 7.92E-08 & $2.70 \mathrm{E}-07$ & 3.37E-09 & $3.43 \mathrm{E}-07$ \\
\hline $\mathrm{Pm}-143$ & 1.69E-05 & $9.02 \mathrm{E}-08$ & $4.99 \mathrm{E}-06$ & 1.12E-05 & 5.27E-07 & $1.13 \mathrm{E}-05$ \\
\hline $\mathrm{Pm}-144$ & 2.57E-05 & $4.59 \mathrm{E}-07$ & $2.53 \mathrm{E}-05$ & $0.00 \mathrm{E}+00$ & $0.00 \mathrm{E}+00$ & $4.59 \mathrm{E}-07$ \\
\hline $\mathrm{Pm}-145$ & $2.88 \mathrm{E}-05$ & 4.81E-09 & $4.60 \mathrm{E}-07$ & $2.70 \mathrm{E}-05$ & 1.27E-06 & $2.70 \mathrm{E}-05$ \\
\hline $\mathrm{Pm}-146$ & 1.79E-04 & $2.21 \mathrm{E}-07$ & $1.24 \mathrm{E}-05$ & 1.59E-04 & $7.48 \mathrm{E}-06$ & $1.59 \mathrm{E}-04$ \\
\hline $\mathrm{Pm}-147$ & 4.56E-05 & 1.06E-12 & $6.52 \mathrm{E}-11$ & 4.36E-05 & $2.05 \mathrm{E}-06$ & 4.36E-05 \\
\hline $\mathrm{Pm}-148$ & 5.83E-06 & $1.72 \mathrm{E}-07$ & $5.66 \mathrm{E}-06$ & $0.00 \mathrm{E}+00$ & $0.00 \mathrm{E}+00$ & 1.72E-07 \\
\hline $\mathrm{Pm}-148 \mathrm{~m}$ & $3.16 \mathrm{E}-05$ & 5.97E-07 & $3.10 \mathrm{E}-05$ & $0.00 \mathrm{E}+00$ & $0.00 \mathrm{E}+00$ & 5.97E-07 \\
\hline $\mathrm{Pm}-149$ & $3.64 \mathrm{E}-06$ & 3.37E-09 & 8.04E-08 & $3.47 \mathrm{E}-06$ & $9.21 \mathrm{E}-08$ & $3.47 \mathrm{E}-06$ \\
\hline Pm-151 & 1.46E-06 & $9.53 \mathrm{E}-08$ & $1.36 \mathrm{E}-06$ & $3.14 \mathrm{E}-11$ & $3.50 \mathrm{E}-11$ & $9.53 \mathrm{E}-08$ \\
\hline Sm-146 & 9.65E-02 & $0.00 \mathrm{E}+00$ & $0.00 \mathrm{E}+00$ & $9.22 \mathrm{E}-02$ & $4.35 \mathrm{E}-03$ & $9.22 \mathrm{E}-02$ \\
\hline Sm-147 & 8.76E-02 & $0.00 \mathrm{E}+00$ & $0.00 \mathrm{E}+00$ & 8.37E-02 & $3.94 \mathrm{E}-03$ & 8.37E-02 \\
\hline Sm-151 & 3.54E-05 & $2.67 \mathrm{E}-13$ & $8.37 \mathrm{E}-11$ & $3.38 \mathrm{E}-05$ & 1.59E-06 & $3.38 \mathrm{E}-05$ \\
\hline Sm-153 & 2.79E-06 & 1.46E-08 & $4.11 \mathrm{E}-07$ & $2.30 \mathrm{E}-06$ & $5.76 \mathrm{E}-08$ & $2.31 \mathrm{E}-06$ \\
\hline Eu-150b & 3.04E-04 & $0.00 \mathrm{E}+00$ & $0.00 \mathrm{E}+00$ & 2.91E-04 & 1.37E-05 & 2.91E-04 \\
\hline Eu-152 & 2.66E-04 & $3.44 \mathrm{E}-07$ & $1.79 \mathrm{E}-05$ & 2.37E-04 & $1.12 \mathrm{E}-05$ & $2.37 \mathrm{E}-04$ \\
\hline Eu-152m & 1.23E-06 & $8.76 \mathrm{E}-08$ & $3.88 \mathrm{E}-07$ & $7.52 \mathrm{E}-07$ & $6.15 \mathrm{E}-09$ & $8.40 \mathrm{E}-07$ \\
\hline Eu-154 & 3.47E-04 & $3.79 \mathrm{E}-07$ & $1.93 \mathrm{E}-05$ & 3.12E-04 & 1.47E-05 & $3.12 \mathrm{E}-04$ \\
\hline Eu-155 & $1.15 \mathrm{E}-06$ & $1.66 \mathrm{E}-08$ & $1.14 \mathrm{E}-06$ & $0.00 \mathrm{E}+00$ & $0.00 \mathrm{E}+00$ & $1.66 \mathrm{E}-08$ \\
\hline Eu-156 & 1.76E-05 & $4.27 \mathrm{E}-07$ & $1.72 \mathrm{E}-05$ & $0.00 \mathrm{E}+00$ & $0.00 \mathrm{E}+00$ & $4.27 \mathrm{E}-07$ \\
\hline Gd-148 & 1.02E-01 & $0.00 \mathrm{E}+00$ & $0.00 \mathrm{E}+00$ & $9.74 \mathrm{E}-02$ & $4.59 \mathrm{E}-03$ & $9.74 \mathrm{E}-02$ \\
\hline Gd-152 & 7.62E-02 & $0.00 \mathrm{E}+00$ & $0.00 \mathrm{E}+00$ & $7.28 \mathrm{E}-02$ & $3.43 \mathrm{E}-03$ & $7.28 \mathrm{E}-02$ \\
\hline Gd-153 & 2.02E-06 & $2.58 \mathrm{E}-08$ & 1.99E-06 & $0.00 \mathrm{E}+00$ & $0.00 \mathrm{E}+00$ & $2.58 \mathrm{E}-08$ \\
\hline
\end{tabular}


Table 2. Ground release, Class D, $4 \mathrm{~m} / \mathrm{s}$ wind, $1 \mathrm{~km}$, no rain, continued.

\begin{tabular}{|c|c|c|c|c|c|c|}
\hline Nuclide & $\begin{array}{c}\text { 7-day } \\
\text { Total } \\
(1+2+3+4)\end{array}$ & $\begin{array}{c}\text { Cloud } \\
\text { shine } \\
(1)\end{array}$ & $\begin{array}{l}\text { Ground } \\
\text { shine } \\
(2)\end{array}$ & $\begin{array}{c}\text { Inhalation } \\
\text { (3) }\end{array}$ & $\begin{array}{l}\text { Resus- } \\
\text { pension } \\
(4)\end{array}$ & $\begin{array}{c}\text { Plume } \\
\text { Passage } \\
(1)+(3)\end{array}$ \\
\hline Gd-159 & $1.40 \mathrm{E}-06$ & 1.05E-08 & $1.08 \mathrm{E}-07$ & 1.27E-06 & $1.78 \mathrm{E}-08$ & $1.28 \mathrm{E}-06$ \\
\hline Tb-157 & 7.52E-06 & $7.44 \mathrm{E}-10$ & 7.41E-08 & 7.11E-06 & $3.35 \mathrm{E}-07$ & 7.11E-06 \\
\hline Tb-158 & $2.64 \mathrm{E}-04$ & $0.00 \mathrm{E}+00$ & $0.00 \mathrm{E}+00$ & $2.52 \mathrm{E}-04$ & 1.19E-05 & $2.52 \mathrm{E}-04$ \\
\hline Tb-160 & 4.26E-05 & $3.27 \mathrm{E}-07$ & $1.63 \mathrm{E}-05$ & $2.48 \mathrm{E}-05$ & 1.14E-06 & $2.51 \mathrm{E}-05$ \\
\hline Tb-161 & 4.02E-06 & $0.00 \mathrm{E}+00$ & $0.00 \mathrm{E}+00$ & $3.87 \mathrm{E}-06$ & $1.48 \mathrm{E}-07$ & $3.87 \mathrm{E}-06$ \\
\hline Dy-157 & 4.93E-07 & $9.03 \mathrm{E}-08$ & $4.03 \mathrm{E}-07$ & $3.83 \mathrm{E}-12$ & $1.77 \mathrm{E}-12$ & $9.03 \mathrm{E}-08$ \\
\hline Dy-159 & 2.32E-06 & $0.00 \mathrm{E}+00$ & $0.00 \mathrm{E}+00$ & $2.21 \mathrm{E}-06$ & 1.03E-07 & $2.21 \mathrm{E}-06$ \\
\hline Dy-165 & $1.26 \mathrm{E}-08$ & $5.24 \mathrm{E}-09$ & $7.36 \mathrm{E}-09$ & $0.00 \mathrm{E}+00$ & $0.00 \mathrm{E}+00$ & $5.24 \mathrm{E}-09$ \\
\hline Dy-166 & 4.13E-05 & 9.33E-09 & $5.82 \mathrm{E}-07$ & 2.33E-05 & 1.74E-05 & $2.33 \mathrm{E}-05$ \\
\hline Но-164 & 4.06E-09 & $0.00 \mathrm{E}+00$ & $0.00 \mathrm{E}+00$ & 4.05E-09 & $2.02 \mathrm{E}-12$ & $4.05 \mathrm{E}-09$ \\
\hline Ho-164m & $1.88 \mathrm{E}-08$ & $0.00 \mathrm{E}+00$ & $0.00 \mathrm{E}+00$ & $1.88 \mathrm{E}-08$ & 1.46E-11 & $1.88 \mathrm{E}-08$ \\
\hline Ho-166 & 8.49E-04 & $7.98 \mathrm{E}-09$ & $1.02 \mathrm{E}-07$ & 8.33E-04 & $1.51 \mathrm{E}-05$ & 8.33E-04 \\
\hline Ho-166m & $2.69 \mathrm{E}-05$ & 4.79E-07 & $2.64 \mathrm{E}-05$ & $0.00 \mathrm{E}+00$ & $0.00 \mathrm{E}+00$ & $4.79 \mathrm{E}-07$ \\
\hline Er-169 & $2.45 \mathrm{E}-06$ & $5.55 \mathrm{E}-13$ & $6.20 \mathrm{E}-11$ & $2.36 \mathrm{E}-06$ & $9.47 \mathrm{E}-08$ & $2.36 \mathrm{E}-06$ \\
\hline Er-171 & 1.24E-06 & $9.76 \mathrm{E}-08$ & 4.01E-07 & $7.40 \mathrm{E}-07$ & 5.22E-09 & 8.38E-07 \\
\hline Tm-170 & 3.07E-05 & $1.35 \mathrm{E}-09$ & $9.76 \mathrm{E}-08$ & $2.92 \mathrm{E}-05$ & 1.36E-06 & $2.92 \mathrm{E}-05$ \\
\hline Tm-171 & 1.07E-05 & $1.52 \mathrm{E}-10$ & $1.25 \mathrm{E}-08$ & 1.02E-05 & 4.82E-07 & $1.02 \mathrm{E}-05$ \\
\hline Yb-169 & 5.35E-06 & $8.31 \mathrm{E}-08$ & $5.27 \mathrm{E}-06$ & $0.00 \mathrm{E}+00$ & $0.00 \mathrm{E}+00$ & $8.31 \mathrm{E}-08$ \\
\hline$Y b-175$ & 4.14E-07 & 1.13E-08 & 4.03E-07 & $0.00 \mathrm{E}+00$ & $0.00 \mathrm{E}+00$ & $1.13 \mathrm{E}-08$ \\
\hline Lu-174 & 4.43E-05 & $0.00 \mathrm{E}+00$ & $0.00 \mathrm{E}+00$ & $4.23 \mathrm{E}-05$ & $1.99 \mathrm{E}-06$ & $4.23 \mathrm{E}-05$ \\
\hline Lu-174m & $2.80 \mathrm{E}-05$ & $0.00 \mathrm{E}+00$ & $0.00 \mathrm{E}+00$ & $2.67 \mathrm{E}-05$ & $1.25 \mathrm{E}-06$ & 2.67E-05 \\
\hline Lu-176 & 7.79E-04 & $0.00 \mathrm{E}+00$ & $0.00 \mathrm{E}+00$ & 7.44E-04 & $3.51 \mathrm{E}-05$ & 7.44E-04 \\
\hline Lu-176m & 3.78E-07 & $0.00 \mathrm{E}+00$ & $0.00 \mathrm{E}+00$ & $3.76 \mathrm{E}-07$ & 1.34E-09 & 3.76E-07 \\
\hline Lu-177 & 3.52E-06 & $1.01 \mathrm{E}-08$ & $4.51 \mathrm{E}-07$ & $2.95 \mathrm{E}-06$ & $1.12 \mathrm{E}-07$ & $2.96 \mathrm{E}-06$ \\
\hline Lu-177m & $5.75 \mathrm{E}-05$ & $2.88 \mathrm{E}-07$ & 1.73E-05 & $3.81 \mathrm{E}-05$ & $1.78 \mathrm{E}-06$ & 3.84E-05 \\
\hline Lu-178 & $2.05 \mathrm{E}-08$ & $0.00 \mathrm{E}+00$ & $0.00 \mathrm{E}+00$ & $2.05 \mathrm{E}-08$ & $9.99 \mathrm{E}-12$ & $2.05 \mathrm{E}-08$ \\
\hline Lu-178m & 9.89E-09 & $0.00 \mathrm{E}+00$ & $0.00 \mathrm{E}+00$ & $9.89 \mathrm{E}-09$ & $3.86 \mathrm{E}-12$ & $9.89 \mathrm{E}-09$ \\
\hline $\mathrm{Hf}-175$ & $4.01 \mathrm{E}-06$ & $0.00 \mathrm{E}+00$ & $0.00 \mathrm{E}+00$ & $3.84 \mathrm{E}-06$ & 1.77E-07 & 3.84E-06 \\
\hline $\mathrm{Hf}-177 \mathrm{~m}$ & $5.89 \mathrm{E}-08$ & $0.00 \mathrm{E}+00$ & $0.00 \mathrm{E}+00$ & $5.89 \mathrm{E}-08$ & $5.15 \mathrm{E}-11$ & $5.89 \mathrm{E}-08$ \\
\hline $\mathrm{Hf}-178 \mathrm{~m}$ & $6.18 \mathrm{E}-04$ & $0.00 \mathrm{E}+00$ & $0.00 \mathrm{E}+00$ & 5.91E-04 & $2.78 \mathrm{E}-05$ & 5.91E-04 \\
\hline $\mathrm{Hf}-179 \mathrm{~m}$ & $1.11 \mathrm{E}-05$ & $0.00 \mathrm{E}+00$ & $0.00 \mathrm{E}+00$ & 1.07E-05 & 4.73E-07 & 1.07E-05 \\
\hline Hf-181 & $2.22 \mathrm{E}-05$ & $1.59 \mathrm{E}-07$ & 8.75E-06 & $1.28 \mathrm{E}-05$ & $5.80 \mathrm{E}-07$ & $1.30 \mathrm{E}-05$ \\
\hline Hf-182 & $1.01 \mathrm{E}-03$ & $1.05 \mathrm{E}-10$ & $4.11 \mathrm{E}-07$ & $9.61 \mathrm{E}-04$ & $4.54 \mathrm{E}-05$ & $9.61 \mathrm{E}-04$ \\
\hline Hf-183 & 1.17E-07 & $0.00 \mathrm{E}+00$ & $0.00 \mathrm{E}+00$ & $1.15 \mathrm{E}-07$ & 1.79E-09 & 1.15E-07 \\
\hline Ta-179 & $5.75 \mathrm{E}-06$ & $0.00 \mathrm{E}+00$ & $0.00 \mathrm{E}+00$ & $5.50 \mathrm{E}-06$ & $2.59 \mathrm{E}-07$ & $5.50 \mathrm{E}-06$ \\
\hline Ta-180m & 9.67E-08 & $0.00 \mathrm{E}+00$ & $0.00 \mathrm{E}+00$ & $9.60 \mathrm{E}-08$ & $6.96 \mathrm{E}-10$ & $9.60 \mathrm{E}-08$ \\
\hline Ta-182 & $7.00 \mathrm{E}-05$ & 3.89E-07 & 1.92E-05 & $4.82 \mathrm{E}-05$ & $2.24 \mathrm{E}-06$ & $4.86 \mathrm{E}-05$ \\
\hline Ta-182m & 7.77E-09 & $3.50 \mathrm{E}-11$ & $1.84 \mathrm{E}-09$ & $5.68 \mathrm{E}-09$ & $2.15 \mathrm{E}-10$ & 5.72E-09 \\
\hline Ta-183 & $5.67 \mathrm{E}-06$ & $0.00 \mathrm{E}+00$ & $0.00 \mathrm{E}+00$ & $5.47 \mathrm{E}-06$ & 1.95E-07 & 5.47E-06 \\
\hline Ta-184 & $1.24 \mathrm{E}-06$ & $0.00 \mathrm{E}+00$ & $0.00 \mathrm{E}+00$ & $1.23 \mathrm{E}-06$ & $9.48 \mathrm{E}-09$ & $1.23 \mathrm{E}-06$ \\
\hline Ta-185 & $6.81 \mathrm{E}-08$ & $2.18 \mathrm{E}-15$ & $2.17 \mathrm{E}-13$ & $6.80 \mathrm{E}-08$ & $7.61 \mathrm{E}-11$ & $6.80 \mathrm{E}-08$ \\
\hline
\end{tabular}


Table 2. Ground release, Class D, $4 \mathrm{~m} / \mathrm{s}$ wind, $1 \mathrm{~km}$, no rain, continued.

\begin{tabular}{|c|c|c|c|c|c|c|}
\hline Nuclide & $\begin{array}{c}\text { 7-day } \\
\text { Total } \\
(1+2+3+4)\end{array}$ & $\begin{array}{l}\text { Cloud } \\
\text { shine } \\
(1)\end{array}$ & $\begin{array}{c}\text { Ground } \\
\text { shine } \\
(2)\end{array}$ & $\begin{array}{c}\text { Inhalation } \\
(3)\end{array}$ & $\begin{array}{l}\text { Resus- } \\
\text { pension } \\
(4)\end{array}$ & $\begin{array}{c}\text { Plume } \\
\text { Passage } \\
(1)+(3)\end{array}$ \\
\hline Ta-186 & $6.43 \mathrm{E}-10$ & $0.00 \mathrm{E}+00$ & $0.00 \mathrm{E}+00$ & $6.43 \mathrm{E}-10$ & $1.16 \mathrm{E}-13$ & $6.43 \mathrm{E}-10$ \\
\hline W-179 & 1.41E-09 & $0.00 \mathrm{E}+00$ & $0.00 \mathrm{E}+00$ & 1.40E-09 & 1.09E-11 & 1.40E-09 \\
\hline W-181 & 8.68E-07 & $9.48 \mathrm{E}-09$ & $7.26 \mathrm{E}-07$ & $1.27 \mathrm{E}-07$ & 5.91E-09 & $1.36 \mathrm{E}-07$ \\
\hline W-185 & 9.82E-07 & $8.08 \mathrm{E}-12$ & $4.82 \mathrm{E}-10$ & $9.38 \mathrm{E}-07$ & $4.33 \mathrm{E}-08$ & $9.38 \mathrm{E}-07$ \\
\hline W-187 & $2.42 \mathrm{E}-06$ & 1.37E-07 & $1.59 \mathrm{E}-06$ & $6.78 \mathrm{E}-07$ & $1.14 \mathrm{E}-08$ & $8.15 \mathrm{E}-07$ \\
\hline W-188 & $6.58 \mathrm{E}-06$ & 1.26E-09 & $8.56 \mathrm{E}-07$ & $5.40 \mathrm{E}-06$ & $3.30 \mathrm{E}-07$ & $5.40 \mathrm{E}-06$ \\
\hline $\mathrm{Re}-182 \mathrm{~b}$ & 1.32E-05 & $5.15 \mathrm{E}-07$ & $1.27 \mathrm{E}-05$ & $0.00 \mathrm{E}+00$ & $0.00 \mathrm{E}+00$ & $5.15 \mathrm{E}-07$ \\
\hline $\operatorname{Re}-182 a$ & $2.28 \mathrm{E}-06$ & 3.39E-07 & 1.95E-06 & $0.00 \mathrm{E}+00$ & $0.00 \mathrm{E}+00$ & $3.39 \mathrm{E}-07$ \\
\hline $\operatorname{Re}-184$ & $1.96 \mathrm{E}-05$ & $2.68 \mathrm{E}-07$ & $1.36 \mathrm{E}-05$ & $5.48 \mathrm{E}-06$ & $2.48 \mathrm{E}-07$ & $5.75 \mathrm{E}-06$ \\
\hline $\mathrm{Re}-184 \mathrm{~m}$ & 1.75E-05 & $1.14 \mathrm{E}-07$ & $7.12 \mathrm{E}-06$ & $9.77 \mathrm{E}-06$ & $4.64 \mathrm{E}-07$ & $9.88 \mathrm{E}-06$ \\
\hline $\operatorname{Re}-186$ & 3.79E-06 & $5.87 \mathrm{E}-09$ & $2.15 \mathrm{E}-07$ & $3.46 \mathrm{E}-06$ & 1.13E-07 & $3.47 \mathrm{E}-06$ \\
\hline $\mathrm{Re}-186 \mathrm{~m}$ & 3.30E-05 & $4.82 \mathrm{E}-11$ & $1.68 \mathrm{E}-07$ & $3.13 \mathrm{E}-05$ & $1.53 \mathrm{E}-06$ & $3.13 \mathrm{E}-05$ \\
\hline $\operatorname{Re}-187$ & $6.26 \mathrm{E}-08$ & $0.00 \mathrm{E}+00$ & $0.00 \mathrm{E}+00$ & $5.98 \mathrm{E}-08$ & 2.82E-09 & $5.98 \mathrm{E}-08$ \\
\hline $\operatorname{Re}-188$ & 2.64E-06 & 1.66E-08 & $1.42 \mathrm{E}-07$ & $2.45 \mathrm{E}-06$ & $3.24 \mathrm{E}-08$ & $2.47 \mathrm{E}-06$ \\
\hline $\mathrm{Re}-188 \mathrm{~m}$ & $4.96 \mathrm{E}-08$ & $2.79 \mathrm{E}-10$ & $2.63 \mathrm{E}-09$ & $4.60 \mathrm{E}-08$ & $6.02 \mathrm{E}-10$ & $4.63 \mathrm{E}-08$ \\
\hline $\mathrm{Re}-189$ & 1.39E-06 & $0.00 \mathrm{E}+00$ & $0.00 \mathrm{E}+00$ & 1.36E-06 & $2.34 \mathrm{E}-08$ & $1.36 \mathrm{E}-06$ \\
\hline Os-185 & $1.16 \mathrm{E}-05$ & $2.10 \mathrm{E}-07$ & 1.14E-05 & $0.00 \mathrm{E}+00$ & $0.00 \mathrm{E}+00$ & $2.10 \mathrm{E}-07$ \\
\hline Os-189m & $3.48 \mathrm{E}-08$ & $0.00 \mathrm{E}+00$ & $0.00 \mathrm{E}+00$ & $3.46 \mathrm{E}-08$ & 1.93E-10 & $3.46 \mathrm{E}-08$ \\
\hline Os-190m & $6.55 \mathrm{E}-09$ & $5.23 \mathrm{E}-09$ & $1.32 \mathrm{E}-09$ & $0.00 \mathrm{E}+00$ & $0.00 \mathrm{E}+00$ & $5.23 \mathrm{E}-09$ \\
\hline Os-191 & 5.64E-06 & $2.00 \mathrm{E}-08$ & $1.16 \mathrm{E}-06$ & 4.27E-06 & 1.83E-07 & $4.29 \mathrm{E}-06$ \\
\hline Os-191m & $6.20 \mathrm{E}-08$ & 1.17E-09 & $4.74 \mathrm{E}-08$ & 8.34E-09 & $5.08 \mathrm{E}-09$ & $9.51 \mathrm{E}-09$ \\
\hline Os-193 & $3.11 \mathrm{E}-07$ & $1.90 \mathrm{E}-08$ & $2.92 \mathrm{E}-07$ & $0.00 \mathrm{E}+00$ & $0.00 \mathrm{E}+00$ & $1.90 \mathrm{E}-08$ \\
\hline Os-194 & 9.57E-04 & 1.03E-09 & $1.26 \mathrm{E}-06$ & $9.12 \mathrm{E}-04$ & $4.31 \mathrm{E}-05$ & $9.12 \mathrm{E}-04$ \\
\hline $\mid r-190$ & 2.57E-05 & $4.11 \mathrm{E}-07$ & $1.93 \mathrm{E}-05$ & $5.74 \mathrm{E}-06$ & $2.39 \mathrm{E}-07$ & $6.15 \mathrm{E}-06$ \\
\hline Ir-190m & 1.23E-07 & 7.35E-09 & $8.61 \mathrm{E}-08$ & $2.82 \mathrm{E}-08$ & $9.97 \mathrm{E}-10$ & $3.56 \mathrm{E}-08$ \\
\hline Ir-190n & $1.25 \mathrm{E}-08$ & $2.59 \mathrm{E}-10$ & $1.13 \mathrm{E}-08$ & $8.19 \mathrm{E}-10$ & $1.23 \mathrm{E}-10$ & $1.08 \mathrm{E}-09$ \\
\hline Ir-192 & 4.63E-05 & $2.40 \mathrm{E}-07$ & $1.35 \mathrm{E}-05$ & $3.11 \mathrm{E}-05$ & $1.44 \mathrm{E}-06$ & $3.13 \mathrm{E}-05$ \\
\hline Ir-192m & 6.56E-04 & $1.00 \mathrm{E}-10$ & $4.50 \mathrm{E}-07$ & $6.26 \mathrm{E}-04$ & $2.95 \mathrm{E}-05$ & $6.26 \mathrm{E}-04$ \\
\hline Ir-194 & 3.96E-06 & $2.60 \mathrm{E}-08$ & $2.43 \mathrm{E}-07$ & 3.64E-06 & $5.25 \mathrm{E}-08$ & 3.67E-06 \\
\hline Ir-194m & 1.03E-04 & $6.91 \mathrm{E}-07$ & $3.86 \mathrm{E}-05$ & $6.13 \mathrm{E}-05$ & $2.86 \mathrm{E}-06$ & $6.20 \mathrm{E}-05$ \\
\hline Pt-191 & 2.49E-06 & $8.11 \mathrm{E}-08$ & $2.41 \mathrm{E}-06$ & $0.00 \mathrm{E}+00$ & $0.00 \mathrm{E}+00$ & 8.11E-08 \\
\hline Pt-193 & 6.69E-08 & $1.29 \mathrm{E}-11$ & 8.98E-09 & $5.53 \mathrm{E}-08$ & 2.61E-09 & $5.53 \mathrm{E}-08$ \\
\hline Pt-193m & 5.31E-07 & $2.80 \mathrm{E}-09$ & $1.25 \mathrm{E}-07$ & 3.90E-07 & 1.33E-08 & 3.93E-07 \\
\hline Pt-195m & 7.97E-07 & $1.88 \mathrm{E}-08$ & $7.78 \mathrm{E}-07$ & $0.00 \mathrm{E}+00$ & $0.00 \mathrm{E}+00$ & $1.88 \mathrm{E}-08$ \\
\hline Pt-197 & $6.53 \mathrm{E}-07$ & $6.36 \mathrm{E}-09$ & $6.74 \mathrm{E}-08$ & $5.72 \mathrm{E}-07$ & 7.97E-09 & $5.78 \mathrm{E}-07$ \\
\hline Pt-197m & 1.37E-07 & $1.42 \mathrm{E}-08$ & 2.07E-08 & $1.01 \mathrm{E}-07$ & $8.04 \mathrm{E}-10$ & 1.15E-07 \\
\hline Au-194 & 6.67E-06 & $3.25 \mathrm{E}-07$ & 5.34E-06 & 9.83E-07 & $2.25 \mathrm{E}-08$ & $1.31 \mathrm{E}-06$ \\
\hline Au-195 & 1.57E-06 & $2.21 \mathrm{E}-08$ & $1.55 \mathrm{E}-06$ & $0.00 \mathrm{E}+00$ & $0.00 \mathrm{E}+00$ & $2.21 \mathrm{E}-08$ \\
\hline Au-195m & $3.02 \mathrm{E}-12$ & $4.27 \mathrm{E}-14$ & $2.98 \mathrm{E}-12$ & $0.00 \mathrm{E}+00$ & $0.00 \mathrm{E}+00$ & $4.27 \mathrm{E}-14$ \\
\hline Au-198 & 8.30E-06 & 1.17E-07 & $3.16 \mathrm{E}-06$ & $4.88 \mathrm{E}-06$ & $1.41 \mathrm{E}-07$ & $5.00 \mathrm{E}-06$ \\
\hline Au-198m & 7.93E-06 & $1.34 \mathrm{E}-09$ & $1.89 \mathrm{E}-06$ & 5.83E-06 & 2.12E-07 & 5.83E-06 \\
\hline
\end{tabular}


Table 2. Ground release, Class D, $4 \mathrm{~m} / \mathrm{s}$ wind, $1 \mathrm{~km}$, no rain, continued.

\begin{tabular}{|c|c|c|c|c|c|c|}
\hline Nuclide & $\begin{array}{c}\text { 7-day } \\
\text { Total } \\
(1+2+3+4)\end{array}$ & $\begin{array}{l}\text { Cloud } \\
\text { shine } \\
(1)\end{array}$ & $\begin{array}{c}\text { Ground } \\
\text { shine } \\
(2)\end{array}$ & $\begin{array}{c}\text { Inhalation } \\
(3)\end{array}$ & $\begin{array}{l}\text { Resus- } \\
\text { pension } \\
(4)\end{array}$ & $\begin{array}{c}\text { Plume } \\
\text { Passage } \\
(1)+(3)\end{array}$ \\
\hline Au-199 & 8.50E-07 & $2.61 \mathrm{E}-08$ & $8.24 \mathrm{E}-07$ & $0.00 \mathrm{E}+00$ & $0.00 \mathrm{E}+00$ & $2.61 \mathrm{E}-08$ \\
\hline $\mathrm{Hg}-194$ & 5.70E-05 & $6.16 \mathrm{E}-09$ & $1.14 \mathrm{E}-05$ & 4.36E-05 & $2.08 \mathrm{E}-06$ & $4.36 \mathrm{E}-05$ \\
\hline $\mathrm{Hg}-197$ & 1.34E-06 & $1.81 \mathrm{E}-08$ & 5.87E-07 & 7.17E-07 & $2.07 \mathrm{E}-08$ & $7.35 \mathrm{E}-07$ \\
\hline $\mathrm{Hg}-197 \mathrm{~m}$ & 1.79E-06 & $2.59 \mathrm{E}-08$ & $5.20 \mathrm{E}-07$ & $1.22 \mathrm{E}-06$ & $2.53 \mathrm{E}-08$ & $1.25 \mathrm{E}-06$ \\
\hline $\mathrm{Hg}-199 \mathrm{~m}$ & 2.19E-08 & $0.00 \mathrm{E}+00$ & $0.00 \mathrm{E}+00$ & $2.19 \mathrm{E}-08$ & $1.59 \mathrm{E}-11$ & $2.19 \mathrm{E}-08$ \\
\hline $\mathrm{Hg}-203$ & 1.09E-05 & $6.67 \mathrm{E}-08$ & $3.81 \mathrm{E}-06$ & $6.67 \mathrm{E}-06$ & 3.04E-07 & $6.74 \mathrm{E}-06$ \\
\hline TI-200 & 4.92E-06 & $3.82 \mathrm{E}-07$ & $4.54 \mathrm{E}-06$ & $0.00 \mathrm{E}+00$ & $0.00 \mathrm{E}+00$ & $3.82 \mathrm{E}-07$ \\
\hline TI-201 & 8.74E-07 & $2.50 \mathrm{E}-08$ & 8.49E-07 & $0.00 \mathrm{E}+00$ & $0.00 \mathrm{E}+00$ & $2.50 \mathrm{E}-08$ \\
\hline TI-202 & 7.84E-06 & $1.34 \mathrm{E}-07$ & $6.57 \mathrm{E}-06$ & $1.09 \mathrm{E}-06$ & $4.52 \mathrm{E}-08$ & $1.22 \mathrm{E}-06$ \\
\hline TI-204 & $3.10 \mathrm{E}-06$ & $2.96 \mathrm{E}-10$ & $2.06 \mathrm{E}-08$ & $2.94 \mathrm{E}-06$ & $1.39 \mathrm{E}-07$ & $2.94 \mathrm{E}-06$ \\
\hline TI-206 & $2.36 \mathrm{E}-13$ & $0.00 \mathrm{E}+00$ & $0.00 \mathrm{E}+00$ & $2.36 \mathrm{E}-13$ & $1.72 \mathrm{E}-17$ & $2.36 \mathrm{E}-13$ \\
\hline TI-207 & $7.04 \mathrm{E}-14$ & $5.90 \mathrm{E}-14$ & $1.13 \mathrm{E}-14$ & $0.00 \mathrm{E}+00$ & $0.00 \mathrm{E}+00$ & $5.90 \mathrm{E}-14$ \\
\hline TI-208 & $6.74 \mathrm{E}-13$ & $5.89 \mathrm{E}-13$ & $8.48 \mathrm{E}-14$ & $0.00 \mathrm{E}+00$ & $0.00 \mathrm{E}+00$ & $5.89 \mathrm{E}-13$ \\
\hline TI-209 & $9.84 \mathrm{E}-10$ & $1.07 \mathrm{E}-15$ & $1.56 \mathrm{E}-16$ & $9.81 \mathrm{E}-10$ & $3.11 \mathrm{E}-12$ & $9.81 \mathrm{E}-10$ \\
\hline $\mathrm{Pb}-202$ & $1.34 \mathrm{E}-04$ & $3.40 \mathrm{E}-10$ & $1.40 \mathrm{E}-06$ & $1.26 \mathrm{E}-04$ & 5.96E-06 & $1.26 \mathrm{E}-04$ \\
\hline $\mathrm{Pb}-203$ & $2.65 \mathrm{E}-06$ & $8.60 \mathrm{E}-08$ & $2.13 \mathrm{E}-06$ & $4.18 \mathrm{E}-07$ & $1.10 \mathrm{E}-08$ & $5.04 \mathrm{E}-07$ \\
\hline $\mathrm{Pb}-205$ & $3.26 \mathrm{E}-06$ & $1.67 \mathrm{E}-11$ & $1.22 \mathrm{E}-08$ & $3.10 \mathrm{E}-06$ & 1.46E-07 & $3.10 \mathrm{E}-06$ \\
\hline $\mathrm{Pb}-209$ & 8.63E-08 & $0.00 \mathrm{E}+00$ & $0.00 \mathrm{E}+00$ & $8.60 \mathrm{E}-08$ & $2.73 \mathrm{E}-10$ & $8.60 \mathrm{E}-08$ \\
\hline $\mathrm{Pb}-210$ & 1.63E-02 & $3.85 \mathrm{E}-10$ & $4.75 \mathrm{E}-08$ & $1.55 \mathrm{E}-02$ & 7.35E-04 & $1.55 \mathrm{E}-02$ \\
\hline $\mathrm{Pb}-211$ & $1.31 \mathrm{E}-08$ & 8.96E-09 & 4.17E-09 & $0.00 \mathrm{E}+00$ & $0.00 \mathrm{E}+00$ & 8.96E-09 \\
\hline $\mathrm{Pb}-212$ & $2.36 \mathrm{E}-06$ & $2.66 \mathrm{E}-07$ & $2.09 \mathrm{E}-06$ & $0.00 \mathrm{E}+00$ & $0.00 \mathrm{E}+00$ & $2.66 \mathrm{E}-07$ \\
\hline $\mathrm{Pb}-214$ & 2.53E-07 & 1.67E-07 & $6.47 \mathrm{E}-08$ & $2.02 \mathrm{E}-08$ & 1.67E-09 & $1.87 \mathrm{E}-07$ \\
\hline $\mathrm{Bi}-206$ & $3.70 \mathrm{E}-05$ & 9.97E-07 & $3.60 \mathrm{E}-05$ & $0.00 \mathrm{E}+00$ & $0.00 \mathrm{E}+00$ & 9.97E-07 \\
\hline $\mathrm{Bi}-207$ & 4.83E-05 & 4.64E-07 & $2.43 \mathrm{E}-05$ & $2.25 \mathrm{E}-05$ & 1.06E-06 & $2.30 \mathrm{E}-05$ \\
\hline $\mathrm{Bi}-210$ & $2.32 \mathrm{E}-04$ & $5.76 \mathrm{E}-16$ & $1.78 \mathrm{E}-12$ & $2.20 \mathrm{E}-04$ & $1.19 \mathrm{E}-05$ & $2.20 \mathrm{E}-04$ \\
\hline Bi-210m & 9.01E-03 & $0.00 \mathrm{E}+00$ & $0.00 \mathrm{E}+00$ & 8.61E-03 & 4.06E-04 & $8.61 \mathrm{E}-03$ \\
\hline $\mathrm{Bi}-211$ & $5.71 \mathrm{E}-14$ & $4.79 \mathrm{E}-14$ & $9.20 \mathrm{E}-15$ & $0.00 \mathrm{E}+00$ & $0.00 \mathrm{E}+00$ & $4.79 \mathrm{E}-14$ \\
\hline $\mathrm{Bi}-212$ & 3.55E-07 & $2.36 \mathrm{E}-07$ & 1.19E-07 & $0.00 \mathrm{E}+00$ & $0.00 \mathrm{E}+00$ & $2.36 \mathrm{E}-07$ \\
\hline $\mathrm{Bi}-213$ & 4.57E-08 & $2.09 \mathrm{E}-08$ & $1.10 \mathrm{E}-08$ & $1.38 \mathrm{E}-08$ & 7.37E-11 & 3.47E-08 \\
\hline $\mathrm{Bi}-214$ & 8.94E-08 & $5.06 \mathrm{E}-08$ & $1.41 \mathrm{E}-08$ & $2.35 \mathrm{E}-08$ & $1.25 \mathrm{E}-09$ & 7.41E-08 \\
\hline Po-210 & 1.02E-02 & $2.59 \mathrm{E}-12$ & $1.36 \mathrm{E}-10$ & $9.77 \mathrm{E}-03$ & $4.56 \mathrm{E}-04$ & $9.77 \mathrm{E}-03$ \\
\hline Po-211 & $0.00 \mathrm{E}+00$ & $0.00 \mathrm{E}+00$ & $0.00 \mathrm{E}+00$ & $0.00 \mathrm{E}+00$ & $0.00 \mathrm{E}+00$ & $0.00 \mathrm{E}+00$ \\
\hline Po-213 & 3.09E-17 & $0.00 \mathrm{E}+00$ & $0.00 \mathrm{E}+00$ & 3.09E-17 & $0.00 \mathrm{E}+00$ & $3.09 \mathrm{E}-17$ \\
\hline Po-214 & $3.79 \mathrm{E}-15$ & $8.98 \mathrm{E}-23$ & $1.11 \mathrm{E}-20$ & $3.62 \mathrm{E}-15$ & $1.71 \mathrm{E}-16$ & $3.62 \mathrm{E}-15$ \\
\hline Po-215 & $1.08 \mathrm{E}-14$ & $7.37 \mathrm{E}-15$ & $3.42 \mathrm{E}-15$ & $0.00 \mathrm{E}+00$ & $0.00 \mathrm{E}+00$ & $7.37 \mathrm{E}-15$ \\
\hline Po-216 & $9.23 \mathrm{E}-12$ & $1.04 \mathrm{E}-12$ & $8.19 \mathrm{E}-12$ & $0.00 \mathrm{E}+00$ & $0.00 \mathrm{E}+00$ & $1.04 \mathrm{E}-12$ \\
\hline Po-218 & 3.05E-08 & $2.02 \mathrm{E}-08$ & 7.98E-09 & $2.14 \mathrm{E}-09$ & $1.90 \mathrm{E}-10$ & $2.23 \mathrm{E}-08$ \\
\hline At-211 & $7.65 \mathrm{E}-07$ & 9.02E-09 & $7.56 \mathrm{E}-07$ & $1.98 \mathrm{E}-11$ & $1.21 \mathrm{E}-10$ & $9.04 \mathrm{E}-09$ \\
\hline At-217 & $5.38 \mathrm{E}-13$ & $2.46 \mathrm{E}-13$ & $1.28 \mathrm{E}-13$ & $1.63 \mathrm{E}-13$ & $8.69 \mathrm{E}-16$ & $4.09 \mathrm{E}-13$ \\
\hline Rn-218 & $8.08 \mathrm{E}-13$ & $1.91 \mathrm{E}-20$ & $2.36 \mathrm{E}-18$ & $7.72 \mathrm{E}-13$ & $3.64 \mathrm{E}-14$ & $7.72 \mathrm{E}-13$ \\
\hline Rn-219 & $2.41 \mathrm{E}-11$ & $1.64 \mathrm{E}-11$ & $7.64 \mathrm{E}-12$ & $0.00 \mathrm{E}+00$ & $0.00 \mathrm{E}+00$ & 1.64E-11 \\
\hline
\end{tabular}


Table 2. Ground release, Class D, $4 \mathrm{~m} / \mathrm{s}$ wind, $1 \mathrm{~km}$, no rain, continued.

\begin{tabular}{|c|c|c|c|c|c|c|}
\hline Nuclide & $\begin{array}{c}\text { 7-day } \\
\text { Total } \\
(1+2+3+4)\end{array}$ & $\begin{array}{l}\text { Cloud } \\
\text { shine } \\
(1)\end{array}$ & $\begin{array}{c}\text { Ground } \\
\text { shine } \\
(2)\end{array}$ & $\begin{array}{c}\text { Inhalation } \\
(3)\end{array}$ & $\begin{array}{l}\text { Resus- } \\
\text { pension } \\
(4)\end{array}$ & $\begin{array}{c}\text { Plume } \\
\text { Passage } \\
(1)+(3)\end{array}$ \\
\hline Rn-220 & 3.42E-09 & $3.81 \mathrm{E}-10$ & $3.04 \mathrm{E}-09$ & $0.00 \mathrm{E}+00$ & $0.00 \mathrm{E}+00$ & $3.81 \mathrm{E}-10$ \\
\hline Rn-222 & 4.95E-07 & 3.07E-07 & $1.72 \mathrm{E}-07$ & 1.30E-08 & 2.74E-09 & $3.20 \mathrm{E}-07$ \\
\hline Fr-221 & 5.12E-09 & $2.45 \mathrm{E}-09$ & $1.29 \mathrm{E}-09$ & 1.37E-09 & $7.85 \mathrm{E}-12$ & $3.82 \mathrm{E}-09$ \\
\hline Fr-223 & 1.12E-05 & $1.82 \mathrm{E}-09$ & $6.28 \mathrm{E}-09$ & 1.07E-05 & $5.08 \mathrm{E}-07$ & 1.07E-05 \\
\hline Ra-222 & $8.78 \mathrm{E}-10$ & $2.08 \mathrm{E}-17$ & $2.56 \mathrm{E}-15$ & $8.38 \mathrm{E}-10$ & $3.97 \mathrm{E}-11$ & $8.38 \mathrm{E}-10$ \\
\hline Ra-223 & 9.66E-03 & $7.40 \mathrm{E}-08$ & $4.18 \mathrm{E}-06$ & $9.27 \mathrm{E}-03$ & 3.84E-04 & $9.27 \mathrm{E}-03$ \\
\hline Ra-224 & 4.17E-03 & $1.39 \mathrm{E}-08$ & $1.22 \mathrm{E}-05$ & $4.02 \mathrm{E}-03$ & 1.30E-04 & $4.02 \mathrm{E}-03$ \\
\hline Ra-225 & 9.15E-03 & 2.03E-09 & $9.12 \mathrm{E}-07$ & 8.72E-03 & $4.29 \mathrm{E}-04$ & $8.72 \mathrm{E}-03$ \\
\hline Ra-226 & 8.30E-02 & $3.11 \mathrm{E}-09$ & $1.16 \mathrm{E}-05$ & 7.92E-02 & 3.73E-03 & $7.92 \mathrm{E}-02$ \\
\hline Ra-228 & 2.01E-02 & $3.21 \mathrm{E}-08$ & $1.39 \mathrm{E}-05$ & 1.92E-02 & $9.44 \mathrm{E}-04$ & $1.92 \mathrm{E}-02$ \\
\hline Ac-225 & $1.01 \mathrm{E}-02$ & $4.43 \mathrm{E}-08$ & $3.03 \mathrm{E}-06$ & $9.67 \mathrm{E}-03$ & 3.93E-04 & $9.67 \mathrm{E}-03$ \\
\hline Ac-227 & $1.51 \mathrm{E}+00$ & $2.49 \mathrm{E}-10$ & $3.17 \mathrm{E}-07$ & $1.45 \mathrm{E}+00$ & 6.83E-02 & $1.45 \mathrm{E}+00$ \\
\hline Ac-228 & $1.48 \mathrm{E}-04$ & $2.50 \mathrm{E}-07$ & $7.21 \mathrm{E}-07$ & $1.41 \mathrm{E}-04$ & $6.59 \mathrm{E}-06$ & $1.41 \mathrm{E}-04$ \\
\hline Th-226 & 3.47E-08 & 1.26E-09 & $5.62 \mathrm{E}-10$ & $3.10 \mathrm{E}-08$ & 1.93E-09 & 3.23E-08 \\
\hline Th-227 & 1.90E-02 & $3.06 \mathrm{E}-08$ & $2.54 \mathrm{E}-06$ & $1.82 \mathrm{E}-02$ & $8.40 \mathrm{E}-04$ & $1.82 \mathrm{E}-02$ \\
\hline Th-228 & 4.00E-01 & $6.12 \mathrm{E}-10$ & $1.14 \mathrm{E}-06$ & $3.82 \mathrm{E}-01$ & $1.80 \mathrm{E}-02$ & $3.82 \mathrm{E}-01$ \\
\hline Th-229 & $2.03 E+00$ & $2.51 \mathrm{E}-08$ & $1.78 \mathrm{E}-06$ & $1.94 \mathrm{E}+00$ & $9.14 \mathrm{E}-02$ & $1.94 \mathrm{E}+00$ \\
\hline Th-230 & 3.07E-01 & $1.14 \mathrm{E}-10$ & $1.46 \mathrm{E}-08$ & 2.93E-01 & $1.38 \mathrm{E}-02$ & $2.93 \mathrm{E}-01$ \\
\hline Th-231 & 1.09E-06 & $3.28 \mathrm{E}-09$ & $6.55 \mathrm{E}-08$ & $1.00 \mathrm{E}-06$ & $2.02 \mathrm{E}-08$ & $1.00 \mathrm{E}-06$ \\
\hline Th-232 & $1.35 \mathrm{E}+00$ & $5.44 \mathrm{E}-11$ & $2.60 \mathrm{E}-08$ & $1.29 \mathrm{E}+00$ & $6.09 \mathrm{E}-02$ & $1.29 \mathrm{E}+00$ \\
\hline Th-234 & $4.14 \mathrm{E}-05$ & 5.91E-09 & $3.99 \mathrm{E}-07$ & 3.92E-05 & $1.74 \mathrm{E}-06$ & $3.92 \mathrm{E}-05$ \\
\hline Pa-230 & 9.55E-06 & 1.98E-07 & $9.35 \mathrm{E}-06$ & $2.91 \mathrm{E}-10$ & $6.59 \mathrm{E}-10$ & $1.98 \mathrm{E}-07$ \\
\hline Pa-231 & $1.01 \mathrm{E}+00$ & $8.78 \mathrm{E}-09$ & $5.75 \mathrm{E}-07$ & $9.60 \mathrm{E}-01$ & $4.53 \mathrm{E}-02$ & $9.60 \mathrm{E}-01$ \\
\hline Pa-232 & 8.23E-05 & 8.97E-17 & $6.21 \mathrm{E}-13$ & 7.96E-05 & 2.61E-06 & 7.96E-05 \\
\hline Pa-233 & 1.43E-05 & $6.20 \mathrm{E}-08$ & $3.46 \mathrm{E}-06$ & 1.03E-05 & 4.59E-07 & $1.04 \mathrm{E}-05$ \\
\hline Pa-234 & $2.24 \mathrm{E}-06$ & $5.30 \mathrm{E}-07$ & $1.71 \mathrm{E}-06$ & $4.85 \mathrm{E}-11$ & $1.93 \mathrm{E}-11$ & $5.30 \mathrm{E}-07$ \\
\hline Pa-234m & $9.89 \mathrm{E}-12$ & $2.01 \mathrm{E}-12$ & $6.47 \mathrm{E}-12$ & $1.35 \mathrm{E}-12$ & $6.35 \mathrm{E}-14$ & $3.36 \mathrm{E}-12$ \\
\hline U-230 & 4.79E-07 & $4.30 \mathrm{E}-09$ & $3.14 \mathrm{E}-07$ & $2.70 \mathrm{E}-08$ & 1.33E-07 & $3.13 \mathrm{E}-08$ \\
\hline U-231 & 8.53E-07 & 1.99E-08 & $8.21 \mathrm{E}-07$ & 4.11E-09 & 7.57E-09 & $2.40 \mathrm{E}-08$ \\
\hline U-232 & $7.80 \mathrm{E}-01$ & $7.73 \mathrm{E}-11$ & $1.93 \mathrm{E}-08$ & $7.45 \mathrm{E}-01$ & $3.52 \mathrm{E}-02$ & $7.45 \mathrm{E}-01$ \\
\hline U-233 & $1.58 \mathrm{E}-01$ & $6.97 \mathrm{E}-11$ & 8.02E-09 & $1.51 \mathrm{E}-01$ & $7.10 \mathrm{E}-03$ & $1.51 \mathrm{E}-01$ \\
\hline U-234 & $1.55 \mathrm{E}-01$ & $4.45 \mathrm{E}-11$ & $1.30 \mathrm{E}-08$ & $1.48 \mathrm{E}-01$ & $6.98 \mathrm{E}-03$ & $1.48 \mathrm{E}-01$ \\
\hline U-235 & 1.42E-01 & 4.49E-08 & $2.98 \mathrm{E}-06$ & 1.36E-01 & $6.39 \mathrm{E}-03$ & 1.36E-01 \\
\hline U-236 & 1.46E-01 & $3.51 \mathrm{E}-11$ & $1.18 \mathrm{E}-08$ & $1.40 \mathrm{E}-01$ & $6.59 \mathrm{E}-03$ & $1.40 \mathrm{E}-01$ \\
\hline U-237 & 1.87E-06 & $3.89 \mathrm{E}-08$ & $1.83 \mathrm{E}-06$ & $2.21 \mathrm{E}-11$ & $4.51 \mathrm{E}-11$ & $3.89 \mathrm{E}-08$ \\
\hline U-238 & $1.38 \mathrm{E}-01$ & $3.75 \mathrm{E}-11$ & $5.16 \mathrm{E}-08$ & 1.32E-01 & $6.21 \mathrm{E}-03$ & 1.32E-01 \\
\hline U-239 & $3.52 \mathrm{E}-08$ & 2.33E-09 & 9.89E-09 & $2.25 \mathrm{E}-08$ & $4.61 \mathrm{E}-10$ & $2.48 \mathrm{E}-08$ \\
\hline U-240 & $4.68 \mathrm{E}-06$ & $9.41 \mathrm{E}-08$ & 6.44E-07 & $3.90 \mathrm{E}-06$ & $4.79 \mathrm{E}-08$ & 3.99E-06 \\
\hline Np-235 & $7.63 \mathrm{E}-08$ & $5.84 \mathrm{E}-10$ & 7.57E-08 & $5.10 \mathrm{E}-14$ & $1.24 \mathrm{E}-13$ & $5.84 \mathrm{E}-10$ \\
\hline $\mathrm{Np}-236 \mathrm{a}$ & 1.19E-01 & $3.81 \mathrm{E}-08$ & 2.53E-06 & $1.13 \mathrm{E}-01$ & $5.34 \mathrm{E}-03$ & 1.13E-01 \\
\hline Np-236b & $4.47 \mathrm{E}-06$ & $1.44 \mathrm{E}-08$ & $1.80 \mathrm{E}-07$ & 2.17E-06 & $2.11 \mathrm{E}-06$ & $2.18 \mathrm{E}-06$ \\
\hline
\end{tabular}


Table 2. Ground release, Class D, $4 \mathrm{~m} / \mathrm{s}$ wind, $1 \mathrm{~km}$, no rain, continued.

\begin{tabular}{|c|c|c|c|c|c|c|}
\hline Nuclide & $\begin{array}{c}\text { 7-day } \\
\text { Total } \\
(1+2+3+4)\end{array}$ & $\begin{array}{c}\text { Cloud } \\
\text { shine } \\
(1)\end{array}$ & $\begin{array}{l}\text { Ground } \\
\text { shine } \\
(2)\end{array}$ & $\begin{array}{c}\text { Inhalation } \\
(3)\end{array}$ & \begin{tabular}{|c|} 
Resus- \\
pension \\
$(4)$
\end{tabular} & $\begin{array}{c}\text { Plume } \\
\text { Passage } \\
(1)+(3)\end{array}$ \\
\hline $\mathrm{Np}-237$ & 5.87E-01 & $6.75 \mathrm{E}-09$ & 8.39E-07 & 5.61E-01 & 2.64E-02 & 5.61E-01 \\
\hline Np-238 & 4.36E-06 & 1.66E-07 & $3.36 \mathrm{E}-06$ & $3.35 \mathrm{E}-07$ & 4.97E-07 & 5.01E-07 \\
\hline Np-239 & 3.74E-06 & 4.83E-08 & $1.29 \mathrm{E}-06$ & 2.33E-06 & $6.57 \mathrm{E}-08$ & $2.38 \mathrm{E}-06$ \\
\hline $\mathrm{Np}-240$ & 2.96E-07 & $1.75 \mathrm{E}-07$ & 1.17E-07 & $3.55 \mathrm{E}-09$ & $3.33 \mathrm{E}-10$ & $1.79 \mathrm{E}-07$ \\
\hline Np-240m & 1.14E-09 & $2.41 \mathrm{E}-10$ & $5.31 \mathrm{E}-11$ & $8.12 \mathrm{E}-10$ & $3.84 \mathrm{E}-11$ & $1.05 \mathrm{E}-09$ \\
\hline Pu-236 & 1.62E-01 & $3.47 \mathrm{E}-11$ & $1.59 \mathrm{E}-08$ & $1.55 \mathrm{E}-01$ & $7.30 \mathrm{E}-03$ & $1.55 \mathrm{E}-01$ \\
\hline Pu-237 & $9.15 \mathrm{E}-07$ & $1.39 \mathrm{E}-08$ & $9.01 \mathrm{E}-07$ & $2.22 \mathrm{E}-11$ & $5.24 \mathrm{E}-11$ & $1.39 \mathrm{E}-08$ \\
\hline Pu-238 & 3.67E-01 & $2.58 \mathrm{E}-11$ & $1.38 \mathrm{E}-08$ & $3.50 \mathrm{E}-01$ & $1.65 \mathrm{E}-02$ & $3.50 \mathrm{E}-01$ \\
\hline Pu-239 & 3.96E-01 & $2.39 \mathrm{E}-11$ & $6.09 \mathrm{E}-09$ & $3.78 \mathrm{E}-01$ & $1.78 \mathrm{E}-02$ & $3.78 \mathrm{E}-01$ \\
\hline Pu-240 & 3.96E-01 & $2.52 \mathrm{E}-11$ & $1.32 \mathrm{E}-08$ & $3.79 \mathrm{E}-01$ & $1.79 \mathrm{E}-02$ & $3.79 \mathrm{E}-01$ \\
\hline Pu-241 & $6.71 \mathrm{E}-03$ & $5.44 \mathrm{E}-15$ & $2.55 \mathrm{E}-11$ & 6.41E-03 & $3.02 \mathrm{E}-04$ & $6.41 \mathrm{E}-03$ \\
\hline Pu-242 & $3.77 \mathrm{E}-01$ & $2.14 \mathrm{E}-11$ & $1.10 \mathrm{E}-08$ & $3.60 \mathrm{E}-01$ & $1.70 \mathrm{E}-02$ & $3.60 \mathrm{E}-01$ \\
\hline Pu-243 & $3.21 \mathrm{E}-08$ & $5.80 \mathrm{E}-09$ & $1.80 \mathrm{E}-08$ & $6.33 \mathrm{E}-09$ & $1.96 \mathrm{E}-09$ & $1.21 \mathrm{E}-08$ \\
\hline Pu-244 & 3.56E-01 & $4.24 \mathrm{E}-09$ & 4.77E-06 & $3.40 \mathrm{E}-01$ & $1.60 \mathrm{E}-02$ & $3.40 \mathrm{E}-01$ \\
\hline Pu-245 & 7.82E-07 & 1.19E-07 & $6.59 \mathrm{E}-07$ & $9.59 \mathrm{E}-10$ & 3.21E-09 & $1.20 \mathrm{E}-07$ \\
\hline Pu-246 & $1.39 \mathrm{E}-05$ & $2.30 \mathrm{E}-07$ & 1.37E-05 & 4.35E-09 & $2.31 \mathrm{E}-08$ & $2.34 \mathrm{E}-07$ \\
\hline Am-241 & $6.25 \mathrm{E}-01$ & $5.47 \mathrm{E}-09$ & $4.73 \mathrm{E}-07$ & 5.97E-01 & $2.81 \mathrm{E}-02$ & 5.97E-01 \\
\hline Am-242 & 7.08E-05 & $3.83 \mathrm{E}-09$ & 3.92E-08 & $6.76 \mathrm{E}-05$ & $3.17 \mathrm{E}-06$ & 6.76E-05 \\
\hline Am-242m & $6.03 \mathrm{E}-01$ & $3.30 \mathrm{E}-10$ & $3.21 \mathrm{E}-07$ & $5.76 \mathrm{E}-01$ & $2.71 \mathrm{E}-02$ & $5.76 \mathrm{E}-01$ \\
\hline Am-243 & $6.23 \mathrm{E}-01$ & $1.54 \mathrm{E}-08$ & $2.83 \mathrm{E}-06$ & $5.95 \mathrm{E}-01$ & $2.80 \mathrm{E}-02$ & $5.95 \mathrm{E}-01$ \\
\hline Am-244 & 3.54E-06 & $2.25 \mathrm{E}-07$ & 1.10E-06 & 1.43E-06 & 7.87E-07 & $1.66 \mathrm{E}-06$ \\
\hline Am-245 & $2.05 \mathrm{E}-08$ & 6.36E-09 & $8.22 \mathrm{E}-09$ & 5.13E-09 & $7.73 \mathrm{E}-10$ & $1.15 \mathrm{E}-08$ \\
\hline Am-246 & 1.44E-07 & $9.51 \mathrm{E}-08$ & $4.17 \mathrm{E}-08$ & $6.44 \mathrm{E}-09$ & $4.44 \mathrm{E}-10$ & $1.02 \mathrm{E}-07$ \\
\hline Cm-242 & $2.09 \mathrm{E}-02$ & $2.87 \mathrm{E}-11$ & $1.48 \mathrm{E}-08$ & $2.00 \mathrm{E}-02$ & $9.33 \mathrm{E}-04$ & $2.00 \mathrm{E}-02$ \\
\hline $\mathrm{Cm}-243$ & 4.17E-01 & $3.71 \mathrm{E}-08$ & 2.33E-06 & $3.99 \mathrm{E}-01$ & $1.88 \mathrm{E}-02$ & 3.99E-01 \\
\hline Cm-244 & 3.32E-01 & $2.44 \mathrm{E}-11$ & $1.33 \mathrm{E}-08$ & 3.17E-01 & $1.49 \mathrm{E}-02$ & 3.17E-01 \\
\hline Cm-245 & 6.43E-01 & 2.07E-08 & 1.37E-06 & $6.14 \mathrm{E}-01$ & $2.90 \mathrm{E}-02$ & $6.14 \mathrm{E}-01$ \\
\hline Cm-246 & $6.33 \mathrm{E}-01$ & $2.06 \mathrm{E}-11$ & $1.18 \mathrm{E}-08$ & $6.04 \mathrm{E}-01$ & $2.85 \mathrm{E}-02$ & 6.04E-01 \\
\hline $\mathrm{Cm}-247$ & 5.94E-06 & $9.35 \mathrm{E}-08$ & $5.83 \mathrm{E}-06$ & $4.85 \mathrm{E}-10$ & $1.46 \mathrm{E}-08$ & $9.40 \mathrm{E}-08$ \\
\hline Cm-248 & 5.49E-01 & $1.80 \mathrm{E}-11$ & 9.47E-09 & $5.24 \mathrm{E}-01$ & $2.47 \mathrm{E}-02$ & $5.24 \mathrm{E}-01$ \\
\hline Cm-249 & 4.69E-09 & $2.79 \mathrm{E}-09$ & $1.90 \mathrm{E}-09$ & 4.19E-19 & $4.64 \mathrm{E}-17$ & $2.79 \mathrm{E}-09$ \\
\hline Cm-250 & 2.71E-06 & 7.91E-09 & $2.70 \mathrm{E}-06$ & $1.12 \mathrm{E}-12$ & $6.89 \mathrm{E}-10$ & 7.91E-09 \\
\hline Bk-249 & $1.16 \mathrm{E}-10$ & $6.34 \mathrm{E}-14$ & $1.15 \mathrm{E}-10$ & $1.50 \mathrm{E}-14$ & $3.51 \mathrm{E}-13$ & $7.84 \mathrm{E}-14$ \\
\hline Bk-250 & 5.52E-07 & $2.15 \mathrm{E}-07$ & 3.37E-07 & $3.24 \mathrm{E}-14$ & $6.78 \mathrm{E}-13$ & $2.15 \mathrm{E}-07$ \\
\hline Cf-248 & 5.07E-06 & 1.97E-11 & $1.08 \mathrm{E}-08$ & $1.48 \mathrm{E}-06$ & $3.58 \mathrm{E}-06$ & $1.48 \mathrm{E}-06$ \\
\hline Cf-249 & $5.77 \mathrm{E}-06$ & $9.60 \mathrm{E}-08$ & $5.65 \mathrm{E}-06$ & $6.11 \mathrm{E}-09$ & $1.49 \mathrm{E}-08$ & $1.02 \mathrm{E}-07$ \\
\hline Cf-250 & $4.81 \mathrm{E}-08$ & $2.51 \mathrm{E}-11$ & $1.10 \mathrm{E}-08$ & $1.08 \mathrm{E}-08$ & $2.62 \mathrm{E}-08$ & $1.08 \mathrm{E}-08$ \\
\hline Cf-251 & $2.21 \mathrm{E}-06$ & $3.43 \mathrm{E}-08$ & $2.17 \mathrm{E}-06$ & $2.58 \mathrm{E}-20$ & $2.52 \mathrm{E}-18$ & $3.43 \mathrm{E}-08$ \\
\hline Cf-252 & $1.06 \mathrm{E}-08$ & $2.19 \mathrm{E}-11$ & $1.02 \mathrm{E}-08$ & $1.27 \mathrm{E}-10$ & $3.08 \mathrm{E}-10$ & $1.49 \mathrm{E}-10$ \\
\hline Cf-253 & 2.17E-09 & $9.12 \mathrm{E}-12$ & $2.16 \mathrm{E}-09$ & $7.52 \mathrm{E}-22$ & $5.80 \mathrm{E}-17$ & $9.12 \mathrm{E}-12$ \\
\hline Cf-254 & $3.68 \mathrm{E}-13$ & $3.71 \mathrm{E}-15$ & $3.64 \mathrm{E}-13$ & $6.40 \mathrm{E}-24$ & $4.48 \mathrm{E}-19$ & $3.71 \mathrm{E}-15$ \\
\hline
\end{tabular}


Table 2. Ground release, Class D, $4 \mathrm{~m} / \mathrm{s}$ wind, $1 \mathrm{~km}$, no rain, continued.

\begin{tabular}{|c|c|c|c|c|c|c|}
\hline Nuclide & $\begin{array}{c}\text { 7-day } \\
\text { Total } \\
(1+2+3+4)\end{array}$ & $\begin{array}{l}\text { Cloud } \\
\text { shine } \\
\text { (1) }\end{array}$ & $\begin{array}{l}\text { Ground } \\
\text { shine } \\
\text { (2) }\end{array}$ & $\begin{array}{c}\text { Inhalation } \\
(3)\end{array}$ & $\begin{array}{l}\text { Resus- } \\
\text { pension } \\
(4)\end{array}$ & $\begin{array}{c}\text { Plume } \\
\text { Passage } \\
(1)+(3)\end{array}$ \\
\hline Es-253 & $1.03 \mathrm{E}-08$ & $9.77 \mathrm{E}-11$ & $1.02 \mathrm{E}-08$ & $4.95 \mathrm{E}-19$ & $1.23 \mathrm{E}-15$ & $9.77 \mathrm{E}-11$ \\
\hline Es-254 & $1.36 \mathrm{E}-05$ & $5.67 \mathrm{E}-08$ & $1.35 \mathrm{E}-05$ & $2.53 E-15$ & $6.30 \mathrm{E}-12$ & 5.67E-08 \\
\hline Es-254m & $3.11 \mathrm{E}-06$ & $1.65 \mathrm{E}-07$ & $2.95 \mathrm{E}-06$ & $2.46 \mathrm{E}-15$ & $3.71 \mathrm{E}-12$ & $1.65 \mathrm{E}-07$ \\
\hline Fm-254 & $3.17 \mathrm{E}-10$ & $2.59 \mathrm{E}-11$ & $2.90 \mathrm{E}-10$ & $3.24 \mathrm{E}-14$ & $6.82 \mathrm{E}-13$ & $2.59 \mathrm{E}-11$ \\
\hline Fm-255 & $2.03 \mathrm{E}-08$ & $5.93 \mathrm{E}-10$ & $1.97 \mathrm{E}-08$ & $1.57 \mathrm{E}-26$ & $2.32 \mathrm{E}-24$ & $5.93 \mathrm{E}-10$ \\
\hline \multicolumn{3}{|c|}{$\begin{array}{l}\text { NUMBER OF NUCLIDES } \\
\text { EVALUATED: }\end{array}$} & \multicolumn{2}{|r|}{535} & & \\
\hline
\end{tabular}


Table 3. Early dose results $(\mathrm{Sv} / \mathrm{TBq})$ for a $100-\mathrm{m}$ elevated release at an MEI distance of 2.5-2.8 km assuming stability class D, $4 \mathrm{~m} / \mathrm{s}$ wind speed, no rain, and P-G dispersion parameters (sigmas).

\begin{tabular}{|c|c|c|c|c|c|c|}
\hline Juclide & $\begin{array}{c}\text { 7-day } \\
\text { Total } \\
(1+2+3+4)\end{array}$ & $\begin{array}{l}\text { Cloud } \\
\text { shine } \\
(1)\end{array}$ & $\begin{array}{c}\text { Ground } \\
\text { shine } \\
(2)\end{array}$ & $\begin{array}{c}\text { Inhalation } \\
(3)\end{array}$ & $\begin{array}{c}\text { Resus- } \\
\text { pension } \\
(4)\end{array}$ & $\begin{array}{c}\text { Plume } \\
\text { Passage } \\
(1)+(3)\end{array}$ \\
\hline $\mathrm{H}-3$ & $1.94 \mathrm{E}-08$ & $0.00 \mathrm{E}+00$ & $0.00 \mathrm{E}+00$ & $9.98 \mathrm{E}-09$ & $9.40 \mathrm{E}-09$ & $9.98 \mathrm{E}-09$ \\
\hline $\mathrm{Be}-7$ & $1.13 \mathrm{E}-07$ & 2.33E-09 & $7.41 \mathrm{E}-08$ & $3.49 \mathrm{E}-08$ & 1.60E-09 & $3.72 \mathrm{E}-08$ \\
\hline $\mathrm{Be}-10$ & $3.11 \mathrm{E}-05$ & $0.00 \mathrm{E}+00$ & $0.00 \mathrm{E}+00$ & $2.97 \mathrm{E}-05$ & 1.40E-06 & $2.97 \mathrm{E}-05$ \\
\hline C-11 & $4.29 \mathrm{E}-09$ & $4.29 \mathrm{E}-09$ & $0.00 \mathrm{E}+00$ & $0.00 \mathrm{E}+00$ & $0.00 \mathrm{E}+00$ & $4.29 \mathrm{E}-09$ \\
\hline C-14 & 3.07E-09 & $0.00 \mathrm{E}+00$ & $0.00 \mathrm{E}+00$ & 3.07E-09 & $0.00 \mathrm{E}+00$ & 3.07E-09 \\
\hline $\mathrm{N}-13$ & $3.59 \mathrm{E}-10$ & $3.44 \mathrm{E}-10$ & $0.00 \mathrm{E}+00$ & $1.59 \mathrm{E}-11$ & $0.00 \mathrm{E}+00$ & $3.60 \mathrm{E}-10$ \\
\hline $0-15$ & $1.53 \mathrm{E}-18$ & $1.53 \mathrm{E}-18$ & $0.00 \mathrm{E}+00$ & $0.00 \mathrm{E}+00$ & $0.00 \mathrm{E}+00$ & $1.53 \mathrm{E}-18$ \\
\hline $\mathrm{N}-16$ & $0.00 \mathrm{E}+00$ & $0.00 \mathrm{E}+00$ & $0.00 \mathrm{E}+00$ & $0.00 \mathrm{E}+00$ & $0.00 \mathrm{E}+00$ & $0.00 \mathrm{E}+00$ \\
\hline $\mathrm{F}-18$ & $3.96 \mathrm{E}-07$ & 2.92E-08 & $3.61 \mathrm{E}-07$ & 5.94E-09 & $2.18 \mathrm{E}-10$ & $3.51 \mathrm{E}-08$ \\
\hline $\mathrm{Na}-22$ & 4.11E-06 & $1.05 \mathrm{E}-07$ & $3.14 \mathrm{E}-06$ & $8.25 \mathrm{E}-07$ & $3.88 \mathrm{E}-08$ & 9.30E-07 \\
\hline $\mathrm{Na}-24$ & 9.99E-07 & $2.12 \mathrm{E}-07$ & $6.67 \mathrm{E}-07$ & 1.19E-07 & 1.43E-09 & $3.31 \mathrm{E}-07$ \\
\hline Mg-27 & $3.81 \mathrm{E}-10$ & $3.29 \mathrm{E}-10$ & $3.53 \mathrm{E}-11$ & $1.74 \mathrm{E}-11$ & $2.84 \mathrm{E}-15$ & $3.46 \mathrm{E}-10$ \\
\hline Mg-28 & $1.52 \mathrm{E}-06$ & $1.53 \mathrm{E}-07$ & $7.53 \mathrm{E}-07$ & $6.02 \mathrm{E}-07$ & 9.25E-09 & $7.55 \mathrm{E}-07$ \\
\hline Al-26 & 1.22E-05 & 1.37E-07 & $3.75 \mathrm{E}-06$ & 7.95E-06 & $3.75 \mathrm{E}-07$ & 8.09E-06 \\
\hline Al-28 & $2.88 \mathrm{E}-17$ & $2.64 \mathrm{E}-17$ & $2.02 \mathrm{E}-18$ & $3.53 \mathrm{E}-19$ & $0.00 \mathrm{E}+00$ & $2.68 \mathrm{E}-17$ \\
\hline Si-31 & $2.50 \mathrm{E}-08$ & $3.14 \mathrm{E}-11$ & $2.24 \mathrm{E}-11$ & $2.49 \mathrm{E}-08$ & $6.45 \mathrm{E}-11$ & $2.49 \mathrm{E}-08$ \\
\hline Si-32 & 5.74E-06 & $0.00 \mathrm{E}+00$ & $0.00 \mathrm{E}+00$ & $5.48 \mathrm{E}-06$ & 2.61E-07 & $5.48 \mathrm{E}-06$ \\
\hline S-37 & $1.32 \mathrm{E}-13$ & $0.00 \mathrm{E}+00$ & $0.00 \mathrm{E}+00$ & $1.32 \mathrm{E}-13$ & $1.15 \mathrm{E}-17$ & $1.32 \mathrm{E}-13$ \\
\hline $\mathrm{P}-32$ & 5.57E-07 & $0.00 \mathrm{E}+00$ & $0.00 \mathrm{E}+00$ & 5.34E-07 & 2.27E-08 & 5.34E-07 \\
\hline P-33 & 5.92E-08 & $0.00 \mathrm{E}+00$ & $0.00 \mathrm{E}+00$ & 5.67E-08 & 2.52E-09 & 5.67E-08 \\
\hline S-35 & $3.30 \mathrm{E}-08$ & $0.00 \mathrm{E}+00$ & $0.00 \mathrm{E}+00$ & $3.15 \mathrm{E}-08$ & 1.46E-09 & $3.15 \mathrm{E}-08$ \\
\hline Cl-36 & $4.25 \mathrm{E}-07$ & $3.92 \mathrm{E}-16$ & $1.38 \mathrm{E}-12$ & $2.19 \mathrm{E}-07$ & 2.06E-07 & 2.19E-07 \\
\hline $\mathrm{Cl}-38$ & $1.12 \mathrm{E}-07$ & $2.10 \mathrm{E}-08$ & $8.65 \mathrm{E}-08$ & 4.81E-09 & $6.12 \mathrm{E}-11$ & $2.58 \mathrm{E}-08$ \\
\hline Cl-39 & 5.96E-09 & $0.00 \mathrm{E}+00$ & $0.00 \mathrm{E}+00$ & 5.85E-09 & $1.11 \mathrm{E}-10$ & 5.85E-09 \\
\hline $\mathrm{Cl}-40$ & $0.00 \mathrm{E}+00$ & $0.00 \mathrm{E}+00$ & $0.00 \mathrm{E}+00$ & $0.00 \mathrm{E}+00$ & $0.00 \mathrm{E}+00$ & $0.00 \mathrm{E}+00$ \\
\hline Ar-37 & $6.37 \mathrm{E}-14$ & $6.37 \mathrm{E}-14$ & $0.00 \mathrm{E}+00$ & $0.00 \mathrm{E}+00$ & $0.00 \mathrm{E}+00$ & $6.37 \mathrm{E}-14$ \\
\hline Ar-39 & $0.00 \mathrm{E}+00$ & $0.00 \mathrm{E}+00$ & $0.00 \mathrm{E}+00$ & $0.00 \mathrm{E}+00$ & $0.00 \mathrm{E}+00$ & $0.00 \mathrm{E}+00$ \\
\hline Ar-41 & 3.97E-08 & 3.97E-08 & $0.00 \mathrm{E}+00$ & $0.00 \mathrm{E}+00$ & $0.00 \mathrm{E}+00$ & 3.97E-08 \\
\hline $\mathrm{K}-40$ & $1.68 \mathrm{E}-06$ & $7.60 \mathrm{E}-09$ & $2.05 \mathrm{E}-07$ & $1.40 \mathrm{E}-06$ & $6.61 \mathrm{E}-08$ & 1.41E-06 \\
\hline $\mathrm{K}-42$ & $1.84 \mathrm{E}-07$ & $1.27 \mathrm{E}-08$ & $3.72 \mathrm{E}-08$ & 1.33E-07 & 1.37E-09 & 1.46E-07 \\
\hline $\mathrm{K}-43$ & $4.05 \mathrm{E}-07$ & 4.45E-08 & $2.88 \mathrm{E}-07$ & $7.10 \mathrm{E}-08$ & 1.15E-09 & 1.16E-07 \\
\hline Ca-41 & 1.32E-07 & $1.48 \mathrm{E}-13$ & $3.10 \mathrm{E}-11$ & $1.26 \mathrm{E}-07$ & 5.95E-09 & $1.26 \mathrm{E}-07$ \\
\hline Ca-45 & 7.32E-07 & $7.69 \mathrm{E}-19$ & $3.18 \mathrm{E}-16$ & 6.99E-07 & 3.27E-08 & 6.99E-07 \\
\hline Ca-47 & $1.74 \mathrm{E}-06$ & 5.11E-08 & $9.46 \mathrm{E}-07$ & $7.19 \mathrm{E}-07$ & $2.74 \mathrm{E}-08$ & 7.70E-07 \\
\hline Ca-49 & $2.15 \mathrm{E}-09$ & $6.44 \mathrm{E}-10$ & $5.91 \mathrm{E}-11$ & $1.44 \mathrm{E}-09$ & $1.42 \mathrm{E}-12$ & $2.08 \mathrm{E}-09$ \\
\hline Sc-44 & $1.08 \mathrm{E}-06$ & 8.26E-08 & 9.32E-07 & $5.88 \mathrm{E}-08$ & $2.25 \mathrm{E}-09$ & $1.41 \mathrm{E}-07$ \\
\hline Sc-44m & $1.43 \mathrm{E}-05$ & $1.88 \mathrm{E}-08$ & 1.32E-05 & 7.89E-07 & 2.36E-07 & 8.08E-07 \\
\hline Sc-46 & $3.29 \mathrm{E}-05$ & $9.80 \mathrm{E}-08$ & $2.83 \mathrm{E}-05$ & $3.08 \mathrm{E}-06$ & $1.44 \mathrm{E}-06$ & $3.18 \mathrm{E}-06$ \\
\hline
\end{tabular}


Table 3. $100-\mathrm{m}$ elevated release, Class $\mathrm{D}, 4 \mathrm{~m} / \mathrm{s}$ wind, no rain, continued.

\begin{tabular}{|c|c|c|c|c|c|c|}
\hline Nuclide & $\begin{array}{c}\text { 7-day } \\
\text { Total } \\
(1+2+3+4)\end{array}$ & $\begin{array}{c}\text { Cloud } \\
\text { shine } \\
(1)\end{array}$ & $\begin{array}{c}\text { Ground } \\
\text { shine } \\
(2)\end{array}$ & $\begin{array}{c}\text { Inhalation } \\
\text { (3) }\end{array}$ & $\begin{array}{c}\text { Resus- } \\
\text { pension } \\
(4)\end{array}$ & $\begin{array}{c}\text { Plume } \\
\text { Passage } \\
(1)+(3)\end{array}$ \\
\hline Sc-47 & $1.28 \mathrm{E}-06$ & $5.22 \mathrm{E}-09$ & 9.91E-07 & $2.13 \mathrm{E}-07$ & $6.77 \mathrm{E}-08$ & $2.18 \mathrm{E}-07$ \\
\hline Sc-48 & 1.71E-05 & $1.59 \mathrm{E}-07$ & 1.64E-05 & $4.61 \mathrm{E}-07$ & 1.13E-07 & $6.20 \mathrm{E}-07$ \\
\hline Sc-49 & $8.22 \mathrm{E}-09$ & $2.27 \mathrm{E}-11$ & $6.61 \mathrm{E}-11$ & 8.05E-09 & $7.93 \mathrm{E}-11$ & 8.07E-09 \\
\hline Sc-50 & 8.80E-22 & $0.00 \mathrm{E}+00$ & $0.00 \mathrm{E}+00$ & $8.80 \mathrm{E}-22$ & $0.00 \mathrm{E}+00$ & $8.80 \mathrm{E}-22$ \\
\hline Ti-44 & $6.02 \mathrm{E}-05$ & $2.54 \mathrm{E}-08$ & $3.28 \mathrm{E}-05$ & $1.85 \mathrm{E}-05$ & $8.86 \mathrm{E}-06$ & $1.85 \mathrm{E}-05$ \\
\hline Ti-45 & 3.61E-07 & $3.11 \mathrm{E}-08$ & $3.05 \mathrm{E}-07$ & 2.37E-08 & $7.22 \mathrm{E}-10$ & $5.48 \mathrm{E}-08$ \\
\hline Ti-51 & $6.07 \mathrm{E}-12$ & $5.06 \mathrm{E}-12$ & $5.29 \mathrm{E}-13$ & $4.83 \mathrm{E}-13$ & $4.81 \mathrm{E}-17$ & $5.54 \mathrm{E}-12$ \\
\hline$V-48$ & $3.76 \mathrm{E}-05$ & $1.41 \mathrm{E}-07$ & $3.59 \mathrm{E}-05$ & 1.04E-06 & 4.53E-07 & $1.18 \mathrm{E}-06$ \\
\hline V-49 & $5.30 \mathrm{E}-08$ & $4.40 \mathrm{E}-13$ & 1.13E-09 & $3.52 \mathrm{E}-08$ & 1.67E-08 & $3.52 \mathrm{E}-08$ \\
\hline$V-52$ & $2.57 \mathrm{E}-13$ & $2.34 \mathrm{E}-13$ & $1.81 \mathrm{E}-14$ & 4.67E-15 & $2.89 \mathrm{E}-19$ & $2.39 \mathrm{E}-13$ \\
\hline$V-53$ & $8.40 \mathrm{E}-23$ & $0.00 \mathrm{E}+00$ & $0.00 \mathrm{E}+00$ & $8.40 \mathrm{E}-23$ & $0.00 \mathrm{E}+00$ & $8.40 \mathrm{E}-23$ \\
\hline $\mathrm{Cr}-49$ & $2.34 \mathrm{E}-08$ & $1.53 \mathrm{E}-08$ & 4.60E-09 & 3.51E-09 & $2.67 \mathrm{E}-12$ & $1.88 \mathrm{E}-08$ \\
\hline $\mathrm{Cr}-51$ & $8.53 \mathrm{E}-08$ & 1.49E-09 & $4.70 \mathrm{E}-08$ & $3.52 \mathrm{E}-08$ & 1.57E-09 & 3.67E-08 \\
\hline$M n-52$ & 4.09E-06 & $1.68 \mathrm{E}-07$ & $3.28 \mathrm{E}-06$ & $6.14 \mathrm{E}-07$ & $2.24 \mathrm{E}-08$ & 7.82E-07 \\
\hline $\mathrm{Mn}-52 \mathrm{~m}$ & $1.45 \mathrm{E}-08$ & $1.12 \mathrm{E}-08$ & $2.14 \mathrm{E}-09$ & 1.12E-09 & $1.43 \mathrm{E}-12$ & $1.23 \mathrm{E}-08$ \\
\hline$M n-53$ & $5.52 \mathrm{E}-08$ & $8.21 \mathrm{E}-13$ & $2.38 \mathrm{E}-10$ & $5.25 \mathrm{E}-08$ & 2.48E-09 & $5.25 \mathrm{E}-08$ \\
\hline Mn-54 & $2.04 \mathrm{E}-06$ & $4.10 \mathrm{E}-08$ & $1.25 \mathrm{E}-06$ & $7.16 \mathrm{E}-07$ & $3.36 \mathrm{E}-08$ & 7.57E-07 \\
\hline$M n-56$ & $1.48 \mathrm{E}-07$ & $6.35 \mathrm{E}-08$ & $4.42 \mathrm{E}-08$ & $4.02 \mathrm{E}-08$ & $1.02 \mathrm{E}-10$ & $1.04 \mathrm{E}-07$ \\
\hline Mn-57 & $2.23 \mathrm{E}-23$ & $1.81 \mathrm{E}-23$ & $1.42 \mathrm{E}-24$ & $2.81 \mathrm{E}-24$ & $0.00 \mathrm{E}+00$ & $2.09 \mathrm{E}-23$ \\
\hline Fe-52 & $4.58 \mathrm{E}-07$ & $1.30 \mathrm{E}-07$ & $3.19 \mathrm{E}-07$ & $9.59 \mathrm{E}-09$ & $9.94 \mathrm{E}-11$ & $1.40 \mathrm{E}-07$ \\
\hline Fe-55 & $1.41 \mathrm{E}-07$ & $1.08 \mathrm{E}-12$ & $3.32 \mathrm{E}-10$ & $1.35 \mathrm{E}-07$ & $6.33 \mathrm{E}-09$ & 1.35E-07 \\
\hline Fe-59 & $3.00 \mathrm{E}-06$ & $5.78 \mathrm{E}-08$ & $1.56 \mathrm{E}-06$ & $1.33 \mathrm{E}-06$ & $6.04 \mathrm{E}-08$ & $1.39 \mathrm{E}-06$ \\
\hline Fe-60 & $3.10 \mathrm{E}-05$ & $2.07 \mathrm{E}-10$ & $1.14 \mathrm{E}-08$ & $2.96 \mathrm{E}-05$ & $1.40 \mathrm{E}-06$ & 2.96E-05 \\
\hline Co-56 & $5.01 \mathrm{E}-06$ & 1.86E-07 & 4.82E-06 & $0.00 \mathrm{E}+00$ & $0.00 \mathrm{E}+00$ & $1.86 \mathrm{E}-07$ \\
\hline Co-57 & 1.19E-06 & 5.94E-09 & $2.12 \mathrm{E}-07$ & $9.32 \mathrm{E}-07$ & $4.37 \mathrm{E}-08$ & $9.38 \mathrm{E}-07$ \\
\hline Co-58 & $2.68 \mathrm{E}-06$ & $4.75 \mathrm{E}-08$ & $1.43 \mathrm{E}-06$ & $1.15 \mathrm{E}-06$ & $5.30 \mathrm{E}-08$ & $1.20 \mathrm{E}-06$ \\
\hline Co-58m & $1.86 \mathrm{E}-08$ & $2.34 \mathrm{E}-11$ & 7.19E-09 & $1.11 \mathrm{E}-08$ & $3.26 \mathrm{E}-10$ & $1.11 \mathrm{E}-08$ \\
\hline Co-60 & $2.71 \mathrm{E}-05$ & $1.22 \mathrm{E}-07$ & $3.40 \mathrm{E}-06$ & $2.25 \mathrm{E}-05$ & 1.06E-06 & $2.26 \mathrm{E}-05$ \\
\hline Co-60m & $1.06 \mathrm{E}-10$ & $2.33 \mathrm{E}-12$ & $1.31 \mathrm{E}-11$ & $8.70 \mathrm{E}-11$ & $4.00 \mathrm{E}-12$ & 8.93E-11 \\
\hline Co-61 & $1.56 \mathrm{E}-08$ & $2.50 \mathrm{E}-09$ & 1.59E-09 & $1.15 \mathrm{E}-08$ & $1.91 \mathrm{E}-11$ & $1.40 \mathrm{E}-08$ \\
\hline Co-62m & $1.99 \mathrm{E}-10$ & $0.00 \mathrm{E}+00$ & $0.00 \mathrm{E}+00$ & $1.99 \mathrm{E}-10$ & $4.76 \mathrm{E}-14$ & $1.99 \mathrm{E}-10$ \\
\hline $\mathrm{Ni}-56$ & $2.00 \mathrm{E}-06$ & $8.34 \mathrm{E}-08$ & $1.92 \mathrm{E}-06$ & $0.00 \mathrm{E}+00$ & $0.00 \mathrm{E}+00$ & 8.34E-08 \\
\hline $\mathrm{Ni}-57$ & $1.08 \mathrm{E}-06$ & $9.21 \mathrm{E}-08$ & $7.81 \mathrm{E}-07$ & 2.07E-07 & $4.64 \mathrm{E}-09$ & $2.99 \mathrm{E}-07$ \\
\hline $\mathrm{Ni}-59$ & $9.39 \mathrm{E}-08$ & $1.82 \mathrm{E}-12$ & $6.27 \mathrm{E}-10$ & $8.91 \mathrm{E}-08$ & $4.20 \mathrm{E}-09$ & 8.91E-08 \\
\hline $\mathrm{Ni}-63$ & $2.56 \mathrm{E}-07$ & $0.00 \mathrm{E}+00$ & $0.00 \mathrm{E}+00$ & $2.45 \mathrm{E}-07$ & $1.15 \mathrm{E}-08$ & $2.45 \mathrm{E}-07$ \\
\hline $\mathrm{Ni}-65$ & 5.92E-08 & $1.93 \mathrm{E}-08$ & $1.32 \mathrm{E}-08$ & $2.66 \mathrm{E}-08$ & $6.64 \mathrm{E}-11$ & $4.59 \mathrm{E}-08$ \\
\hline Cu-61 & $6.29 \mathrm{E}-08$ & 3.06E-08 & $3.23 \mathrm{E}-08$ & $0.00 \mathrm{E}+00$ & $0.00 \mathrm{E}+00$ & 3.06E-08 \\
\hline Cu-62 & $3.83 \mathrm{E}-10$ & $3.03 E-10$ & $4.38 \mathrm{E}-11$ & $3.68 \mathrm{E}-11$ & $6.19 \mathrm{E}-15$ & $3.40 \mathrm{E}-10$ \\
\hline Cu-64 & 7.71E-08 & 8.36E-09 & $3.08 \mathrm{E}-08$ & $3.75 \mathrm{E}-08$ & $3.96 \mathrm{E}-10$ & $4.59 \mathrm{E}-08$ \\
\hline Cu-66 & $1.43 \mathrm{E}-13$ & $0.00 \mathrm{E}+00$ & $0.00 \mathrm{E}+00$ & $1.43 \mathrm{E}-13$ & $1.26 \mathrm{E}-17$ & $1.43 \mathrm{E}-13$ \\
\hline Cu-67 & $9.38 \mathrm{E}-08$ & 5.47E-09 & 8.83E-08 & $0.00 \mathrm{E}+00$ & $0.00 \mathrm{E}+00$ & 5.47E-09 \\
\hline
\end{tabular}


Table 3. $100-\mathrm{m}$ elevated release, Class $\mathrm{D}, 4 \mathrm{~m} / \mathrm{s}$ wind, no rain, continued.

\begin{tabular}{|c|c|c|c|c|c|c|}
\hline Nuclide & $\begin{array}{c}\text { 7-day } \\
\text { Total } \\
(1+2+3+4)\end{array}$ & $\begin{array}{c}\text { Cloud } \\
\text { shine } \\
(1)\end{array}$ & $\begin{array}{c}\text { Ground } \\
\text { shine } \\
(2)\end{array}$ & $\begin{array}{c}\text { Inhalation } \\
\text { (3) }\end{array}$ & $\begin{array}{c}\text { Resus- } \\
\text { pension } \\
(4)\end{array}$ & $\begin{array}{c}\text { Plume } \\
\text { Passage } \\
(1)+(3)\end{array}$ \\
\hline $\mathrm{Zn}-62$ & $2.43 \mathrm{E}-07$ & $6.33 \mathrm{E}-08$ & $1.74 \mathrm{E}-07$ & $5.35 \mathrm{E}-09$ & $4.38 \mathrm{E}-11$ & 6.87E-08 \\
\hline Zn-63 & 4.34E-09 & $0.00 \mathrm{E}+00$ & $0.00 \mathrm{E}+00$ & 4.34E-09 & $2.83 \mathrm{E}-12$ & 4.34E-09 \\
\hline Zn-65 & $3.08 \mathrm{E}-06$ & $2.83 \mathrm{E}-08$ & $8.13 \mathrm{E}-07$ & $2.13 \mathrm{E}-06$ & $1.00 \mathrm{E}-07$ & $2.16 \mathrm{E}-06$ \\
\hline Zn-69 & $3.08 \mathrm{E}-09$ & $1.19 \mathrm{E}-13$ & $4.48 \mathrm{E}-14$ & 3.08E-09 & $2.98 \mathrm{E}-12$ & 3.08E-09 \\
\hline Zn-69m & $2.00 \mathrm{E}-07$ & $1.84 \mathrm{E}-08$ & $7.50 \mathrm{E}-08$ & $1.05 \mathrm{E}-07$ & $1.21 \mathrm{E}-09$ & $1.23 \mathrm{E}-07$ \\
\hline Zn-71m & $5.08 \mathrm{E}-08$ & $0.00 \mathrm{E}+00$ & $0.00 \mathrm{E}+00$ & 5.06E-08 & $1.91 \mathrm{E}-10$ & 5.06E-08 \\
\hline Ga-66 & $3.79 \mathrm{E}-07$ & $1.21 \mathrm{E}-07$ & $2.58 \mathrm{E}-07$ & $0.00 \mathrm{E}+00$ & $0.00 \mathrm{E}+00$ & $1.21 \mathrm{E}-07$ \\
\hline Ga-67 & $1.34 \mathrm{E}-07$ & $6.83 \mathrm{E}-09$ & 1.27E-07 & $0.00 \mathrm{E}+00$ & $0.00 \mathrm{E}+00$ & 6.83E-09 \\
\hline Ga-68 & $3.99 \mathrm{E}-08$ & $2.17 \mathrm{E}-08$ & 9.07E-09 & $9.13 \mathrm{E}-09$ & $1.05 \mathrm{E}-11$ & $3.08 \mathrm{E}-08$ \\
\hline Ga-70 & $5.12 \mathrm{E}-10$ & $0.00 \mathrm{E}+00$ & $0.00 \mathrm{E}+00$ & $5.12 \mathrm{E}-10$ & $1.86 \mathrm{E}-13$ & $5.12 \mathrm{E}-10$ \\
\hline Ga-72 & 8.04E-07 & 1.33E-07 & 4.47E-07 & $2.20 \mathrm{E}-07$ & $2.52 \mathrm{E}-09$ & 3.53E-07 \\
\hline Ge-68 & $1.48 \mathrm{E}-06$ & 2.31E-08 & $1.45 \mathrm{E}-06$ & 9.71E-09 & $8.73 \mathrm{E}-10$ & $3.28 \mathrm{E}-08$ \\
\hline Ge-69 & 7.79E-08 & $0.00 \mathrm{E}+00$ & $0.00 \mathrm{E}+00$ & 7.62E-08 & 1.73E-09 & 7.62E-08 \\
\hline Ge-71 & $1.45 \mathrm{E}-09$ & $4.29 \mathrm{E}-12$ & 1.44E-09 & $0.00 \mathrm{E}+00$ & $0.00 \mathrm{E}+00$ & $4.29 \mathrm{E}-12$ \\
\hline Ge-75 & 7.05E-09 & $0.00 \mathrm{E}+00$ & $0.00 \mathrm{E}+00$ & 7.04E-09 & $9.83 \mathrm{E}-12$ & 7.04E-09 \\
\hline Ge-77 & $3.52 \mathrm{E}-07$ & $4.78 \mathrm{E}-08$ & 1.53E-07 & 1.49E-07 & 2.09E-09 & 1.97E-07 \\
\hline As-72 & $6.73 \mathrm{E}-07$ & $8.37 \mathrm{E}-08$ & $5.89 \mathrm{E}-07$ & $0.00 \mathrm{E}+00$ & $0.00 \mathrm{E}+00$ & 37E-08 \\
\hline As-73 & $1.43 \mathrm{E}-08$ & $2.12 \mathrm{E}-10$ & $1.40 \mathrm{E}-08$ & $0.00 \mathrm{E}+00$ & $0.00 \mathrm{E}+00$ & $2.12 \mathrm{E}-10$ \\
\hline As-74 & $1.94 \mathrm{E}-06$ & $3.63 \mathrm{E}-08$ & 1.03E-06 & $8.31 \mathrm{E}-07$ & $3.59 \mathrm{E}-08$ & 8.67E-07 \\
\hline As-76 & $6.04 \mathrm{E}-07$ & $2.01 \mathrm{E}-08$ & 1.41E-07 & $4.35 \mathrm{E}-07$ & $7.81 \mathrm{E}-09$ & $4.55 \mathrm{E}-07$ \\
\hline As-77 & $1.29 \mathrm{E}-07$ & $4.00 \mathrm{E}-10$ & $4.38 \mathrm{E}-09$ & $1.21 \mathrm{E}-07$ & $2.76 \mathrm{E}-09$ & $1.21 \mathrm{E}-07$ \\
\hline As-78 & $2.70 \mathrm{E}-08$ & $0.00 \mathrm{E}+00$ & $0.00 \mathrm{E}+00$ & $2.69 \mathrm{E}-08$ & $4.11 \mathrm{E}-11$ & $2.69 \mathrm{E}-08$ \\
\hline Se-73 & 1.45E-07 & 4.57E-08 & $9.98 \mathrm{E}-08$ & $0.00 \mathrm{E}+00$ & $0.00 \mathrm{E}+00$ & 4.57E-08 \\
\hline Se-75 & $1.59 \mathrm{E}-06$ & 1.86E-08 & $6.35 \mathrm{E}-07$ & 8.91E-07 & $4.15 \mathrm{E}-08$ & $9.10 \mathrm{E}-07$ \\
\hline Se-79 & $1.05 \mathrm{E}-06$ & $0.00 \mathrm{E}+00$ & $0.00 \mathrm{E}+00$ & $1.00 \mathrm{E}-06$ & $4.73 \mathrm{E}-08$ & $1.00 \mathrm{E}-06$ \\
\hline $\mathrm{Br}-77$ & $4.11 \mathrm{E}-06$ & $1.45 \mathrm{E}-08$ & $4.10 \mathrm{E}-06$ & $0.00 \mathrm{E}+00$ & $0.00 \mathrm{E}+00$ & $1.45 \mathrm{E}-08$ \\
\hline $\mathrm{Br}-80$ & 1.17E-09 & $2.06 \mathrm{E}-10$ & $7.32 \mathrm{E}-10$ & $2.30 \mathrm{E}-10$ & $1.38 \mathrm{E}-12$ & $4.36 \mathrm{E}-10$ \\
\hline $\mathrm{Br}-80 \mathrm{~m}$ & $1.34 \mathrm{E}-07$ & $3.16 \mathrm{E}-09$ & $9.82 \mathrm{E}-08$ & $2.99 \mathrm{E}-08$ & $2.54 \mathrm{E}-09$ & 3.31E-08 \\
\hline $\mathrm{Br}-82$ & $2.24 \mathrm{E}-05$ & $1.23 \mathrm{E}-07$ & $2.21 \mathrm{E}-05$ & $1.29 \mathrm{E}-07$ & $5.56 \mathrm{E}-08$ & $2.52 \mathrm{E}-07$ \\
\hline $\mathrm{Br}-83$ & 4.52E-09 & $2.46 \mathrm{E}-10$ & $4.28 \mathrm{E}-09$ & $0.00 \mathrm{E}+00$ & $0.00 \mathrm{E}+00$ & $2.46 \mathrm{E}-10$ \\
\hline $\mathrm{Br}-84$ & $9.50 \mathrm{E}-08$ & $2.00 \mathrm{E}-08$ & $7.50 \mathrm{E}-08$ & $0.00 \mathrm{E}+00$ & $0.00 \mathrm{E}+00$ & $2.00 \mathrm{E}-08$ \\
\hline $\mathrm{Kr}-79$ & $1.39 \mathrm{E}-08$ & 1.39E-08 & $0.00 \mathrm{E}+00$ & $0.00 \mathrm{E}+00$ & $0.00 \mathrm{E}+00$ & $1.39 \mathrm{E}-08$ \\
\hline $\mathrm{Kr}-81$ & $5.72 \mathrm{E}-10$ & $5.72 \mathrm{E}-10$ & $0.00 \mathrm{E}+00$ & $0.00 \mathrm{E}+00$ & $0.00 \mathrm{E}+00$ & $5.72 \mathrm{E}-10$ \\
\hline $\mathrm{Kr}-83 \mathrm{~m}$ & $3.40 \mathrm{E}-12$ & $3.40 \mathrm{E}-12$ & $0.00 \mathrm{E}+00$ & $0.00 \mathrm{E}+00$ & $0.00 \mathrm{E}+00$ & $3.40 \mathrm{E}-12$ \\
\hline $\mathrm{Kr}-85$ & $1.26 \mathrm{E}-10$ & $1.26 \mathrm{E}-10$ & $0.00 \mathrm{E}+00$ & $0.00 \mathrm{E}+00$ & $0.00 \mathrm{E}+00$ & $1.26 \mathrm{E}-10$ \\
\hline $\mathrm{Kr}-85 \mathrm{~m}$ & 7.62E-09 & 7.62E-09 & $0.00 \mathrm{E}+00$ & $0.00 \mathrm{E}+00$ & $0.00 \mathrm{E}+00$ & 7.62E-09 \\
\hline $\mathrm{Kr}-87$ & $2.66 \mathrm{E}-08$ & $2.66 \mathrm{E}-08$ & $0.00 \mathrm{E}+00$ & $3.57 \mathrm{E}-22$ & $1.68 \mathrm{E}-23$ & $2.66 \mathrm{E}-08$ \\
\hline $\mathrm{Kr}-88$ & 1.39E-07 & $1.26 \mathrm{E}-07$ & 3.57E-09 & $9.51 \mathrm{E}-09$ & $2.91 \mathrm{E}-12$ & 1.36E-07 \\
\hline $\mathrm{Rb}-81$ & $6.19 \mathrm{E}-08$ & $2.87 \mathrm{E}-08$ & $3.33 \mathrm{E}-08$ & $0.00 \mathrm{E}+00$ & $0.00 \mathrm{E}+00$ & 2.87E-08 \\
\hline $\mathrm{Rb}-82$ & $0.00 \mathrm{E}+00$ & $0.00 \mathrm{E}+00$ & $0.00 \mathrm{E}+00$ & $0.00 \mathrm{E}+00$ & $0.00 \mathrm{E}+00$ & $0.00 \mathrm{E}+00$ \\
\hline $\mathrm{Rb}-83$ & $7.63 \mathrm{E}-07$ & $2.80 \mathrm{E}-08$ & $7.35 \mathrm{E}-07$ & $0.00 \mathrm{E}+00$ & $0.00 \mathrm{E}+00$ & $2.80 \mathrm{E}-08$ \\
\hline
\end{tabular}


Table 3. $100-\mathrm{m}$ elevated release, Class $\mathrm{D}, 4 \mathrm{~m} / \mathrm{s}$ wind, no rain, continued.

\begin{tabular}{|c|c|c|c|c|c|c|}
\hline Nuclide & $\begin{array}{c}\text { 7-day } \\
\text { Total } \\
(1+2+3+4)\end{array}$ & $\begin{array}{c}\text { Cloud } \\
\text { shine } \\
(1)\end{array}$ & $\begin{array}{c}\text { Ground } \\
\text { shine } \\
(2)\end{array}$ & $\begin{array}{c}\text { Inhalation } \\
(3)\end{array}$ & $\begin{array}{c}\text { Resus- } \\
\text { pension } \\
(4)\end{array}$ & $\begin{array}{c}\text { Plume } \\
\text { Passage } \\
(1)+(3)\end{array}$ \\
\hline $\mathrm{Rb}-84$ & 1.80E-06 & $5.18 \mathrm{E}-08$ & $1.22 \mathrm{E}-06$ & 4.99E-07 & $2.24 \mathrm{E}-08$ & 5.51E-07 \\
\hline Rb-86 & $8.58 \mathrm{E}-07$ & 5.50E-09 & $1.15 \mathrm{E}-07$ & 7.06E-07 & $3.07 \mathrm{E}-08$ & 7.12E-07 \\
\hline $\mathrm{Rb}-87$ & $2.59 \mathrm{E}-07$ & $0.00 \mathrm{E}+00$ & $0.00 \mathrm{E}+00$ & $2.48 \mathrm{E}-07$ & 1.17E-08 & $2.48 \mathrm{E}-07$ \\
\hline $\mathrm{Rb}-88$ & $3.86 \mathrm{E}-09$ & $2.70 \mathrm{E}-09$ & $3.15 \mathrm{E}-10$ & $8.39 \mathrm{E}-10$ & $2.57 \mathrm{E}-13$ & 3.54E-09 \\
\hline $\mathrm{Rb}-89$ & $6.12 \mathrm{E}-09$ & 5.40E-09 & $6.10 \mathrm{E}-10$ & $1.03 \mathrm{E}-10$ & $4.92 \mathrm{E}-12$ & 5.50E-09 \\
\hline Sr-82 & $1.55 \mathrm{E}-06$ & $6.14 \mathrm{E}-08$ & 1.49E-06 & $0.00 \mathrm{E}+00$ & $0.00 \mathrm{E}+00$ & $6.14 \mathrm{E}-08$ \\
\hline Sr-85 & $9.77 \mathrm{E}-07$ & $2.85 \mathrm{E}-08$ & $7.45 \mathrm{E}-07$ & $1.95 \mathrm{E}-07$ & 8.96E-09 & $2.24 \mathrm{E}-07$ \\
\hline $\mathrm{Sr}-85 \mathrm{~m}$ & 8.96E-09 & $6.13 \mathrm{E}-09$ & $2.76 \mathrm{E}-09$ & $6.35 \mathrm{E}-11$ & $5.79 \mathrm{E}-12$ & $6.19 \mathrm{E}-09$ \\
\hline Sr-87m & $2.35 \mathrm{E}-08$ & $1.34 \mathrm{E}-08$ & $1.01 \mathrm{E}-08$ & $1.25 \mathrm{E}-24$ & $2.28 \mathrm{E}-25$ & $1.34 \mathrm{E}-08$ \\
\hline Sr-89 & $5.38 \mathrm{E}-07$ & $7.99 \mathrm{E}-12$ & $1.88 \mathrm{E}-10$ & $5.15 \mathrm{E}-07$ & $2.35 \mathrm{E}-08$ & 5.15E-07 \\
\hline Sr-90 & 4.09E-05 & $0.00 \mathrm{E}+00$ & $0.00 \mathrm{E}+00$ & $3.91 \mathrm{E}-05$ & $1.86 \mathrm{E}-06$ & 3.91E-05 \\
\hline Sr-91 & 2.47E-07 & 4.69E-08 & 1.14E-07 & 8.37E-08 & 1.97E-09 & $1.31 \mathrm{E}-07$ \\
\hline Sr-92 & $1.24 \mathrm{E}-07$ & $6.00 \mathrm{E}-08$ & 4.23E-08 & $2.16 \mathrm{E}-08$ & $3.05 \mathrm{E}-10$ & $8.16 \mathrm{E}-08$ \\
\hline$Y-86$ & $8.17 \mathrm{E}-07$ & $2.02 \mathrm{E}-07$ & $6.15 \mathrm{E}-07$ & $0.00 \mathrm{E}+00$ & $0.00 \mathrm{E}+00$ & $2.02 \mathrm{E}-07$ \\
\hline Y-87 & $6.55 \mathrm{E}-07$ & 2.97E-08 & $6.25 \mathrm{E}-07$ & $1.86 \mathrm{E}-25$ & $2.17 \mathrm{E}-24$ & 2.97E-08 \\
\hline Y-88 & $6.68 \mathrm{E}-06$ & $1.66 \mathrm{E}-07$ & $3.54 \mathrm{E}-06$ & $2.83 \mathrm{E}-06$ & 1.32E-07 & 3.00E-06 \\
\hline Y-90 & $9.29 \mathrm{E}-07$ & $0.00 \mathrm{E}+00$ & $0.00 \mathrm{E}+00$ & $9.03 \mathrm{E}-07$ & $2.60 \mathrm{E}-08$ & 9.03E-07 \\
\hline Y-90m & $1.03 \mathrm{E}-07$ & $2.77 \mathrm{E}-08$ & $2.33 \mathrm{E}-08$ & $5.06 \mathrm{E}-08$ & 1.37E-09 & 7.83E-08 \\
\hline Y-91 & $5.19 \mathrm{E}-06$ & $2.08 \mathrm{E}-10$ & 4.63E-09 & 4.96E-06 & $2.28 \mathrm{E}-07$ & 4.96E-06 \\
\hline Y-91m & $1.81 \mathrm{E}-08$ & $1.14 \mathrm{E}-08$ & $3.08 \mathrm{E}-09$ & 3.47E-09 & $1.35 \mathrm{E}-10$ & $1.49 \mathrm{E}-08$ \\
\hline Y-92 & $1.20 \mathrm{E}-07$ & $1.17 \mathrm{E}-08$ & $9.41 \mathrm{E}-09$ & $9.90 \mathrm{E}-08$ & $3.40 \mathrm{E}-10$ & 1.11E-07 \\
\hline Y-93 & $2.82 \mathrm{E}-07$ & 4.97E-09 & $1.03 \mathrm{E}-08$ & $2.65 \mathrm{E}-07$ & $2.31 \mathrm{E}-09$ & 2.70E-07 \\
\hline Y-94 & 1.16E-09 & $0.00 \mathrm{E}+00$ & $0.00 \mathrm{E}+00$ & 1.16E-09 & $3.80 \mathrm{E}-13$ & 1.16E-09 \\
\hline $7 r-86$ & 7.95E-07 & $2.57 \mathrm{E}-08$ & 7.69E-07 & $0.00 \mathrm{E}+00$ & $0.00 \mathrm{E}+00$ & 2.57E-08 \\
\hline $7 r-88$ & $6.85 \mathrm{E}-07$ & $2.14 \mathrm{E}-08$ & $6.61 \mathrm{E}-07$ & $8.85 \mathrm{E}-10$ & 1.95E-09 & $2.23 \mathrm{E}-08$ \\
\hline $\mathrm{Zr}-89$ & $1.18 \mathrm{E}-06$ & $6.64 \mathrm{E}-08$ & $8.72 \mathrm{E}-07$ & $2.31 \mathrm{E}-07$ & 7.21E-09 & 2.97E-07 \\
\hline Zr-93 & 8.84E-06 & $5.07 \mathrm{E}-17$ & $7.41 \mathrm{E}-13$ & $8.45 \mathrm{E}-06$ & $3.98 \mathrm{E}-07$ & $8.45 \mathrm{E}-06$ \\
\hline $\mathrm{Zr}-95$ & $2.85 \mathrm{E}-06$ & $4.26 \mathrm{E}-08$ & $1.12 \mathrm{E}-06$ & $1.61 \mathrm{E}-06$ & $7.54 \mathrm{E}-08$ & 1.65E-06 \\
\hline Zr-97 & $8.51 \mathrm{E}-07$ & $6.59 \mathrm{E}-08$ & $3.24 \mathrm{E}-07$ & $4.55 \mathrm{E}-07$ & 6.09E-09 & $5.21 \mathrm{E}-07$ \\
\hline $\mathrm{Nb}-90$ & 1.19E-06 & 2.47E-07 & $6.82 \mathrm{E}-07$ & $2.54 \mathrm{E}-07$ & 2.99E-09 & 5.01E-07 \\
\hline $\mathrm{Nb}-92 \mathrm{~m}$ & $1.39 \mathrm{E}-06$ & $5.62 \mathrm{E}-08$ & $1.10 \mathrm{E}-06$ & $2.22 \mathrm{E}-07$ & $9.05 \mathrm{E}-09$ & $2.78 \mathrm{E}-07$ \\
\hline $\mathrm{Nb}-93 \mathrm{~m}$ & $3.20 \mathrm{E}-06$ & $7.57 \mathrm{E}-12$ & $1.51 \mathrm{E}-09$ & 3.06E-06 & 1.44E-07 & 3.06E-06 \\
\hline $\mathrm{Nb}-94 \mathrm{~m}$ & $2.34 \mathrm{E}-13$ & $1.28 \mathrm{E}-13$ & $2.29 \mathrm{E}-14$ & $8.25 \mathrm{E}-14$ & $1.07 \mathrm{E}-15$ & $2.11 \mathrm{E}-13$ \\
\hline $\mathrm{Nb}-94$ & $4.29 \mathrm{E}-05$ & $9.14 \mathrm{E}-08$ & $2.31 \mathrm{E}-06$ & $3.86 \mathrm{E}-05$ & $1.82 \mathrm{E}-06$ & 3.87E-05 \\
\hline $\mathrm{Nb}-95$ & $1.72 \mathrm{E}-06$ & $4.43 \mathrm{E}-08$ & 1.06E-06 & $5.90 \mathrm{E}-07$ & $2.66 \mathrm{E}-08$ & $6.34 \mathrm{E}-07$ \\
\hline $\mathrm{Nb}-95 \mathrm{~m}$ & $3.86 \mathrm{E}-07$ & 3.39E-09 & 1.05E-07 & $2.68 \mathrm{E}-07$ & $9.50 \mathrm{E}-09$ & $2.71 \mathrm{E}-07$ \\
\hline $\mathrm{Nb}-96$ & 1.09E-06 & 1.37E-07 & $6.99 \mathrm{E}-07$ & $2.52 \mathrm{E}-07$ & 4.17E-09 & 3.89E-07 \\
\hline $\mathrm{Nb}-97$ & $3.41 \mathrm{E}-08$ & 1.96E-08 & $6.79 \mathrm{E}-09$ & 7.73E-09 & $9.43 \mathrm{E}-12$ & $2.73 \mathrm{E}-08$ \\
\hline $\mathrm{Nb}-97 \mathrm{~m}$ & $4.80 \mathrm{E}-10$ & $2.75 \mathrm{E}-10$ & $9.55 \mathrm{E}-11$ & $1.09 \mathrm{E}-10$ & $1.33 \mathrm{E}-13$ & $3.84 \mathrm{E}-10$ \\
\hline $\mathrm{Nb}-98$ & $8.14 \mathrm{E}-09$ & $0.00 \mathrm{E}+00$ & $0.00 \mathrm{E}+00$ & $8.13 \mathrm{E}-09$ & $7.13 \mathrm{E}-12$ & 8.13E-09 \\
\hline Mo-93 & $3.72 \mathrm{E}-06$ & $4.23 \mathrm{E}-11$ & $8.45 \mathrm{E}-09$ & $3.55 \mathrm{E}-06$ & 1.67E-07 & $3.55 \mathrm{E}-06$ \\
\hline
\end{tabular}


Table 3. $100-\mathrm{m}$ elevated release, Class $\mathrm{D}, 4 \mathrm{~m} / \mathrm{s}$ wind, no rain, continued.

\begin{tabular}{|c|c|c|c|c|c|c|}
\hline Juclide & $\begin{array}{c}\text { 7-day } \\
\text { Total } \\
(1+2+3+4)\end{array}$ & $\begin{array}{c}\text { Cloud } \\
\text { shine } \\
(1)\end{array}$ & $\begin{array}{c}\text { Ground } \\
\text { shine } \\
(2)\end{array}$ & $\begin{array}{c}\text { Inhalation } \\
\text { (3) }\end{array}$ & $\begin{array}{c}\text { Resus- } \\
\text { pension } \\
(4)\end{array}$ & $\begin{array}{c}\text { Plume } \\
\text { Passage } \\
(1)+(3)\end{array}$ \\
\hline Mo-93m & $4.89 \mathrm{E}-08$ & $1.04 \mathrm{E}-18$ & $1.78 \mathrm{E}-15$ & 4.86E-08 & $3.05 \mathrm{E}-10$ & $4.86 \mathrm{E}-08$ \\
\hline Mo-99 & $6.38 \mathrm{E}-07$ & $9.62 \mathrm{E}-09$ & 1.95E-07 & $4.21 \mathrm{E}-07$ & $1.24 \mathrm{E}-08$ & 4.31E-07 \\
\hline Mo-101 & 7.01E-09 & 5.67E-09 & $7.67 \mathrm{E}-10$ & $5.69 \mathrm{E}-10$ & 1.67E-13 & $6.24 \mathrm{E}-09$ \\
\hline Tc-95 & $2.68 \mathrm{E}-07$ & $4.38 \mathrm{E}-08$ & 1.96E-07 & 2.84E-08 & $4.23 \mathrm{E}-10$ & 7.22E-08 \\
\hline Tc-95m & $1.04 \mathrm{E}-06$ & $3.82 \mathrm{E}-08$ & $1.01 \mathrm{E}-06$ & $4.63 \mathrm{E}-11$ & $3.79 \mathrm{E}-11$ & $3.82 \mathrm{E}-08$ \\
\hline Tc-96 & $2.59 \mathrm{E}-06$ & $1.44 \mathrm{E}-07$ & $2.18 \mathrm{E}-06$ & $2.61 \mathrm{E}-07$ & 8.87E-09 & 4.05E-07 \\
\hline Tc-96m & $2.12 \mathrm{E}-08$ & 1.67E-09 & $1.81 \mathrm{E}-08$ & 1.30E-09 & $7.25 \mathrm{E}-11$ & 2.97E-09 \\
\hline Tc-97 & 1.12E-07 & $5.26 \mathrm{E}-11$ & 9.33E-09 & $9.84 \mathrm{E}-08$ & 4.64E-09 & $9.85 \mathrm{E}-08$ \\
\hline Tc-97m & 7.96E-09 & $6.47 \mathrm{E}-11$ & 7.89E-09 & $3.45 \mathrm{E}-18$ & $7.68 \mathrm{E}-18$ & $6.47 \mathrm{E}-11$ \\
\hline Tc-98 & 4.27E-06 & 8.03E-08 & $2.08 \mathrm{E}-06$ & $2.02 \mathrm{E}-06$ & $9.52 \mathrm{E}-08$ & $2.10 \mathrm{E}-06$ \\
\hline Tc-99 & $7.28 \mathrm{E}-07$ & $2.93 \mathrm{E}-14$ & $9.05 \mathrm{E}-13$ & $6.95 \mathrm{E}-07$ & $3.28 \mathrm{E}-08$ & $.95 \mathrm{E}-07$ \\
\hline Tc-99m & 1.97E-08 & $6.45 \mathrm{E}-09$ & 1.03E-08 & 2.94E-09 & $1.64 \mathrm{E}-11$ & 9.39E-09 \\
\hline Tc-101 & $8.44 \mathrm{E}-10$ & $6.58 \mathrm{E}-10$ & $9.21 \mathrm{E}-11$ & $9.38 \mathrm{E}-11$ & $2.29 \mathrm{E}-14$ & $7.52 \mathrm{E}-10$ \\
\hline Ru-97 & 2.43E-07 & $1.28 \mathrm{E}-08$ & $1.82 \mathrm{E}-07$ & 4.63E-08 & $1.38 \mathrm{E}-09$ & 5.91E-08 \\
\hline Ru-103 & 1.67E-06 & $2.71 \mathrm{E}-08$ & $6.88 \mathrm{E}-07$ & $9.10 \mathrm{E}-07$ & $4.13 \mathrm{E}-08$ & 9.37E-07 \\
\hline$u-105$ & 1.37E-07 & $3.76 \mathrm{E}-08$ & 4.49E-08 & 5.35E-08 & $4.86 \mathrm{E}-10$ & $9.11 \mathrm{E}-08$ \\
\hline Ru-106 & $5.09 \mathrm{E}-05$ & $1.18 \mathrm{E}-08$ & $3.05 \mathrm{E}-07$ & 4.83E-05 & $2.27 \mathrm{E}-06$ & 4.83E-05 \\
\hline Rh-101 & $3.98 \mathrm{E}-06$ & $0.00 \mathrm{E}+00$ & $0.00 \mathrm{E}+00$ & $3.80 \mathrm{E}-06$ & 1.79E-07 & $3.80 \mathrm{E}-06$ \\
\hline Rh-101m & $7.88 \mathrm{E}-08$ & $0.00 \mathrm{E}+00$ & $0.00 \mathrm{E}+00$ & 7.62E-08 & $2.62 \mathrm{E}-09$ & 7.62E-08 \\
\hline Rh-102 & $1.25 \mathrm{E}-05$ & $0.00 \mathrm{E}+00$ & $0.00 \mathrm{E}+00$ & 1.19E-05 & 5.61E-07 & 1.19E-05 \\
\hline Rh-102m & $2.33 \mathrm{E}-06$ & $0.00 \mathrm{E}+00$ & $0.00 \mathrm{E}+00$ & $2.23 \mathrm{E}-06$ & $1.04 \mathrm{E}-07$ & $2.23 \mathrm{E}-06$ \\
\hline Rh-103m & $3.75 \mathrm{E}-10$ & $4.76 \mathrm{E}-12$ & $7.90 \mathrm{E}-12$ & $3.62 \mathrm{E}-10$ & $3.46 \mathrm{E}-13$ & 3.67E-10 \\
\hline h-105 & 1.46E-07 & 4.26E-09 & $3.54 \mathrm{E}-08$ & $1.04 \mathrm{E}-07$ & $2.24 \mathrm{E}-09$ & $1.08 \mathrm{E}-07$ \\
\hline $1-106$ & $0.00 \mathrm{E}+00$ & $0.00 \mathrm{E}+00$ & $0.00 \mathrm{E}+00$ & $0.00 \mathrm{E}+00$ & $0.00 \mathrm{E}+00$ & $0.00 \mathrm{E}+00$ \\
\hline $\mathrm{Pd}-103$ & $1.50 \mathrm{E}-08$ & $1.06 \mathrm{E}-10$ & $1.44 \mathrm{E}-08$ & $4.89 \mathrm{E}-10$ & $3.64 \mathrm{E}-11$ & $5.95 \mathrm{E}-10$ \\
\hline Pd-107 & 1.36E-06 & $0.00 \mathrm{E}+00$ & $0.00 \mathrm{E}+00$ & $1.30 \mathrm{E}-06$ & $6.13 \mathrm{E}-08$ & $1.30 \mathrm{E}-06$ \\
\hline Pd-109 & 1.32E-07 & $3.63 \mathrm{E}-11$ & $1.16 \mathrm{E}-10$ & $1.31 \mathrm{E}-07$ & $1.44 \mathrm{E}-09$ & $1.31 \mathrm{E}-07$ \\
\hline $\mathrm{Ag}-106$ & $62 \mathrm{E}-10$ & $0.00 \mathrm{E}+00$ & $0.00 \mathrm{E}+00$ & $6.62 \mathrm{E}-10$ & $E-13$ & $6.62 \mathrm{E}-10$ \\
\hline $\mathrm{Ag}-106 \mathrm{~m}$ & $3.80 \mathrm{E}-06$ & $1.61 \mathrm{E}-07$ & 3.04E-06 & 5.76E-07 & $2.28 \mathrm{E}-08$ & 7.37E-07 \\
\hline $\mathrm{Ag}-108$ & $70 \mathrm{E}-18$ & $1.62 \mathrm{E}-18$ & $1.30 \mathrm{E}-19$ & $9.49 \mathrm{E}-19$ & $0.00 \mathrm{E}+00$ & $2.57 \mathrm{E}-18$ \\
\hline $\mathrm{Ag}-108 \mathrm{~m}$ & 3.12E-05 & 9.17E-08 & $2.43 \mathrm{E}-06$ & 2.73E-05 & $1.29 \mathrm{E}-06$ & 2.74E-05 \\
\hline $\mathrm{Ag}-109 \mathrm{~m}$ & $0.00 \mathrm{E}+00$ & $0.00 \mathrm{E}+00$ & $0.00 \mathrm{E}+00$ & $0.00 \mathrm{E}+00$ & $0.00 \mathrm{E}+00$ & $0.00 \mathrm{E}+00$ \\
\hline $\mathrm{Ag}-110$ & $0.00 \mathrm{E}+00$ & $0.00 \mathrm{E}+00$ & $0.00 \mathrm{E}+00$ & $0.00 \mathrm{E}+00$ & $0.00 \mathrm{E}+00$ & $0.00 \mathrm{E}+00$ \\
\hline $\mathrm{Ag}-110 \mathrm{~m}$ & $1.27 \mathrm{E}-05$ & $1.59 \mathrm{E}-07$ & $3.87 \mathrm{E}-06$ & $8.26 \mathrm{E}-06$ & $3.87 \mathrm{E}-07$ & $8.42 \mathrm{E}-06$ \\
\hline $\mathrm{Ag}-111$ & 7.11E-07 & $1.47 \mathrm{E}-09$ & $3.03 E-08$ & $6.54 \mathrm{E}-07$ & $2.53 \mathrm{E}-08$ & $6.55 \mathrm{E}-07$ \\
\hline Cd-109 & $3.58 \mathrm{E}-06$ & $1.23 \mathrm{E}-10$ & $1.37 \mathrm{E}-08$ & $3.41 \mathrm{E}-06$ & $1.60 \mathrm{E}-07$ & 3.41E-06 \\
\hline Cd-113 & $0.00 \mathrm{E}+00$ & $0.00 \mathrm{E}+00$ & $0.00 \mathrm{E}+00$ & $0.00 \mathrm{E}+00$ & $0.00 \mathrm{E}+00$ & $0.00 \mathrm{E}+00$ \\
\hline $\mathrm{Cd}-113 \mathrm{~m}$ & $4.22 \mathrm{E}-05$ & $0.00 \mathrm{E}+00$ & $0.00 \mathrm{E}+00$ & 4.03E-05 & $1.90 \mathrm{E}-06$ & 4.03E-05 \\
\hline $\mathrm{Cd}-115$ & 7.31E-07 & $1.27 \mathrm{E}-08$ & $2.26 \mathrm{E}-07$ & 4.79E-07 & $1.32 \mathrm{E}-08$ & $4.92 \mathrm{E}-07$ \\
\hline $\mathrm{Cd}-115 \mathrm{~m}$ & $3.02 \mathrm{E}-08$ & $1.28 \mathrm{E}-09$ & $2.89 \mathrm{E}-08$ & $1.85 \mathrm{E}-24$ & $4.07 \mathrm{E}-24$ & $1.28 \mathrm{E}-09$ \\
\hline Cd-117 & $9.20 \mathrm{E}-08$ & $5.11 \mathrm{E}-08$ & $4.08 \mathrm{E}-08$ & $0.00 \mathrm{E}+00$ & $0.00 \mathrm{E}+00$ & $5.11 \mathrm{E}-08$ \\
\hline
\end{tabular}


Table 3. $100-\mathrm{m}$ elevated release, Class $\mathrm{D}, 4 \mathrm{~m} / \mathrm{s}$ wind, no rain, continued.

\begin{tabular}{|c|c|c|c|c|c|c|}
\hline Juclide & $\begin{array}{c}\text { 7-day } \\
\text { Total } \\
(1+2+3+4)\end{array}$ & $\begin{array}{c}\text { Cloud } \\
\text { shine } \\
(1)\end{array}$ & $\begin{array}{c}\text { Ground } \\
\text { shine } \\
(2)\end{array}$ & $\begin{array}{c}\text { Inhalation } \\
(3)\end{array}$ & $\begin{array}{c}\text { Resus- } \\
\text { pension } \\
(4)\end{array}$ & $\begin{array}{c}\text { Plume } \\
\text { Passage } \\
(1)+(3)\end{array}$ \\
\hline $\mathrm{Cd}-117 \mathrm{~m}$ & $2.20 \mathrm{E}-07$ & $1.21 \mathrm{E}-07$ & $9.89 \mathrm{E}-08$ & $0.00 \mathrm{E}+00$ & $0.00 \mathrm{E}+00$ & $1.21 \mathrm{E}-07$ \\
\hline $\ln -111$ & $3.28 \mathrm{E}-07$ & $2.19 \mathrm{E}-08$ & 3.06E-07 & $0.00 \mathrm{E}+00$ & $0.00 \mathrm{E}+00$ & 2.19E-08 \\
\hline In-113m & $1.30 \mathrm{E}-08$ & $8.78 \mathrm{E}-09$ & $4.24 \mathrm{E}-09$ & $0.00 \mathrm{E}+00$ & $0.00 \mathrm{E}+00$ & 8.78E-09 \\
\hline $\ln -114$ & $0.00 \mathrm{E}+00$ & $0.00 \mathrm{E}+00$ & $0.00 \mathrm{E}+00$ & $0.00 \mathrm{E}+00$ & $0.00 \mathrm{E}+00$ & $0.00 \mathrm{E}+00$ \\
\hline In-114m & $6.17 \mathrm{E}-06$ & $6.85 \mathrm{E}-09$ & 1.79E-07 & $5.72 \mathrm{E}-06$ & $2.62 \mathrm{E}-07$ & 5.73E-06 \\
\hline $\ln -115$ & 1.09E-04 & $0.00 \mathrm{E}+00$ & $0.00 \mathrm{E}+00$ & 1.04E-04 & 4.89E-06 & 1.04E-04 \\
\hline In-115m & $3.31 \mathrm{E}-08$ & $7.45 \mathrm{E}-09$ & 8.81E-09 & $1.68 \mathrm{E}-08$ & $7.17 \mathrm{E}-11$ & $2.43 \mathrm{E}-08$ \\
\hline $\mathrm{In}-116 \mathrm{~m}$ & 7.84E-08 & 5.96E-08 & 1.46E-08 & $4.18 \mathrm{E}-09$ & $3.85 \mathrm{E}-12$ & $6.38 \mathrm{E}-08$ \\
\hline $\ln -117$ & 1.63E-08 & $1.30 \mathrm{E}-08$ & $3.29 \mathrm{E}-09$ & $0.00 \mathrm{E}+00$ & $0.00 \mathrm{E}+00$ & $1.30 \mathrm{E}-08$ \\
\hline In-117m & $2.28 \mathrm{E}-08$ & $1.30 \mathrm{E}-08$ & 9.83E-09 & $0.00 \mathrm{E}+00$ & $0.00 \mathrm{E}+00$ & $1.30 \mathrm{E}-08$ \\
\hline Sn-113 & $1.36 \mathrm{E}-06$ & 5.91E-09 & 4.07E-07 & $9.05 \mathrm{E}-07$ & $4.21 \mathrm{E}-08$ & $9.11 \mathrm{E}-07$ \\
\hline Sn-117m & $2.17 \mathrm{E}-07$ & 8.37E-09 & 2.09E-07 & $0.00 \mathrm{E}+00$ & $0.00 \mathrm{E}+00$ & 8.37E-09 \\
\hline Sn-119m & $6.48 \mathrm{E}-07$ & $1.22 \mathrm{E}-10$ & 8.67E-09 & $6.11 \mathrm{E}-07$ & $2.86 \mathrm{E}-08$ & $6.11 \mathrm{E}-07$ \\
\hline Sn-121 & $5.81 \mathrm{E}-08$ & $0.00 \mathrm{E}+00$ & $0.00 \mathrm{E}+00$ & $5.71 \mathrm{E}-08$ & $1.04 \mathrm{E}-09$ & 5.71E-08 \\
\hline Sn-121m & $1.78 \mathrm{E}-06$ & $0.00 \mathrm{E}+00$ & $0.00 \mathrm{E}+00$ & $1.70 \mathrm{E}-06$ & $\mathrm{E}-08$ & 1.70E-06 \\
\hline Sn-123 & $3.48 \mathrm{E}-06$ & $4.02 \mathrm{E}-10$ & 9.34E-09 & $3.32 \mathrm{E}-06$ & $1.54 \mathrm{E}-07$ & 3.32E-06 \\
\hline Sn-125 & $3.51 \mathrm{E}-07$ & $1.80 \mathrm{E}-08$ & $3.33 \mathrm{E}-07$ & $3.86 \mathrm{E}-11$ & $E-11$ & $1.80 \mathrm{E}-08$ \\
\hline Sn-126 & $1.42 \mathrm{E}-05$ & $8.54 \mathrm{E}-08$ & $2.54 \mathrm{E}-06$ & $1.10 \mathrm{E}-05$ & $5.20 \mathrm{E}-07$ & 1.11E-05 \\
\hline Sb-117 & $1.32 \mathrm{E}-08$ & 7.34E-09 & 5.84E-09 & $0.00 \mathrm{E}+00$ & $E+00$ & 7.34E-09 \\
\hline Sb-120b & $4.40 \mathrm{E}-07$ & $0.00 \mathrm{E}+00$ & $0.00 \mathrm{E}+00$ & $4.25 \mathrm{E}-07$ & $1.56 \mathrm{E}-08$ & $4.25 \mathrm{E}-07$ \\
\hline Sb-122 & 8.91E-07 & $2.48 \mathrm{E}-08$ & $3.06 \mathrm{E}-07$ & $5.45 \mathrm{E}-07$ & $1.58 \mathrm{E}-08$ & 5.70E-07 \\
\hline$b-124$ & $5.25 \mathrm{E}-06$ & $1.11 \mathrm{E}-07$ & $2.47 \mathrm{E}-06$ & $2.55 \mathrm{E}-06$ & 1.17E-07 & $2.66 \mathrm{E}-06$ \\
\hline Sb-125 & $1.90 \mathrm{E}-06$ & $2.39 \mathrm{E}-08$ & $6.45 \mathrm{E}-07$ & 1.17E-06 & $5.55 \mathrm{E}-08$ & 1.19E-06 \\
\hline Sb-126 & 4.95E-06 & 1.57E-07 & $3.38 \mathrm{E}-06$ & $1.35 \mathrm{E}-06$ & $5.65 \mathrm{E}-08$ & $1.51 \mathrm{E}-06$ \\
\hline Sb-126m & 9.46E-09 & $7.22 \mathrm{E}-09$ & $1.58 \mathrm{E}-09$ & $6.39 \mathrm{E}-10$ & $8.57 \mathrm{E}-12$ & 7.86E-09 \\
\hline Sb-127 & $6.01 \mathrm{E}-07$ & $3.74 \mathrm{E}-08$ & 5.64E-07 & $0.00 \mathrm{E}+00$ & $0.00 \mathrm{E}+00$ & 3.74E-08 \\
\hline Sb-129 & $2.26 \mathrm{E}-07$ & 7.06E-08 & 7.09E-08 & $8.44 \mathrm{E}-08$ & $4.93 \mathrm{E}-10$ & $1.55 \mathrm{E}-07$ \\
\hline Te-121 & $9.71 \mathrm{E}-07$ & $3.16 \mathrm{E}-08$ & $7.40 \mathrm{E}-07$ & $1.91 \mathrm{E}-07$ & $8.25 \mathrm{E}-09$ & $2.23 \mathrm{E}-07$ \\
\hline Te-121m & $1.78 \mathrm{E}-06$ & $1.18 \mathrm{E}-08$ & $4.26 \mathrm{E}-07$ & $1.28 \mathrm{E}-06$ & $6.03 \mathrm{E}-08$ & $1.29 \mathrm{E}-06$ \\
\hline Te-123 & $4.90 \mathrm{E}-07$ & $1.69 \mathrm{E}-10$ & $1.03 E-08$ & $4.58 \mathrm{E}-07$ & $2.16 \mathrm{E}-08$ & $4.58 \mathrm{E}-07$ \\
\hline Te-123m & $6.41 \mathrm{E}-07$ & 8.01E-09 & $2.32 \mathrm{E}-07$ & 3.83E-07 & $1.78 \mathrm{E}-08$ & 3.91E-07 \\
\hline Te-125m & $7.90 \mathrm{E}-07$ & $5.36 \mathrm{E}-10$ & $2.92 \mathrm{E}-08$ & 7.27E-07 & $3.34 \mathrm{E}-08$ & $7.28 \mathrm{E}-07$ \\
\hline Te-127 & $8.22 \mathrm{E}-10$ & $2.49 \mathrm{E}-10$ & $5.73 \mathrm{E}-10$ & $0.00 \mathrm{E}+00$ & $0.00 \mathrm{E}+00$ & $2.49 \mathrm{E}-10$ \\
\hline Te-127m & $1.63 \mathrm{E}-08$ & $1.94 \mathrm{E}-10$ & $1.61 \mathrm{E}-08$ & $0.00 \mathrm{E}+00$ & $0.00 \mathrm{E}+00$ & $1.94 \mathrm{E}-10$ \\
\hline Te-129 & 9.07E-09 & $1.53 \mathrm{E}-09$ & $5.40 \mathrm{E}-10$ & $6.99 \mathrm{E}-09$ & $8.23 \mathrm{E}-12$ & 8.52E-09 \\
\hline Te-129m & $2.67 \mathrm{E}-06$ & $2.91 \mathrm{E}-09$ & $9.96 \mathrm{E}-08$ & $2.46 \mathrm{E}-06$ & $1.11 \mathrm{E}-07$ & $2.46 \mathrm{E}-06$ \\
\hline Te-131 & $3.13 \mathrm{E}-08$ & $3.58 \mathrm{E}-09$ & 1.67E-08 & $6.62 \mathrm{E}-09$ & 4.33E-09 & $1.02 \mathrm{E}-08$ \\
\hline Te-131m & $1.06 \mathrm{E}-06$ & $8.54 \mathrm{E}-08$ & $6.34 \mathrm{E}-07$ & $3.14 \mathrm{E}-07$ & $2.53 \mathrm{E}-08$ & 3.99E-07 \\
\hline Te-132 & $3.18 \mathrm{E}-06$ & 4.99E-08 & $2.32 \mathrm{E}-06$ & $7.82 \mathrm{E}-07$ & $2.56 \mathrm{E}-08$ & 8.32E-07 \\
\hline Te-133 & $4.28 \mathrm{E}-08$ & 1.46E-09 & $3.18 \mathrm{E}-08$ & 7.31E-09 & 2.24E-09 & 8.77E-09 \\
\hline Te-133m & $1.85 \mathrm{E}-07$ & $5.91 \mathrm{E}-08$ & $1.01 \mathrm{E}-07$ & $1.89 \mathrm{E}-08$ & $6.02 \mathrm{E}-09$ & 7.80E-08 \\
\hline
\end{tabular}


Table 3. $100-\mathrm{m}$ elevated release, Class $\mathrm{D}, 4 \mathrm{~m} / \mathrm{s}$ wind, no rain, continued.

\begin{tabular}{|c|c|c|c|c|c|c|}
\hline Nuclide & $\begin{array}{c}\text { 7-day } \\
\text { Total } \\
(1+2+3+4)\end{array}$ & $\begin{array}{c}\text { Cloud } \\
\text { shine } \\
(1)\end{array}$ & $\begin{array}{c}\text { Ground } \\
\text { shine } \\
(2)\end{array}$ & $\begin{array}{c}\text { Inhalation } \\
\text { (3) }\end{array}$ & $\begin{array}{c}\text { Resus- } \\
\text { pension } \\
(4)\end{array}$ & $\begin{array}{c}\text { Plume } \\
\text { Passage } \\
(1)+(3)\end{array}$ \\
\hline Te-134 & $3.34 \mathrm{E}-07$ & $6.49 \mathrm{E}-08$ & $2.65 \mathrm{E}-07$ & $4.59 \mathrm{E}-09$ & $8.53 \mathrm{E}-11$ & $6.95 \mathrm{E}-08$ \\
\hline $\mid-122$ & $2.72 \mathrm{E}-13$ & $9.49 \mathrm{E}-14$ & $1.77 \mathrm{E}-13$ & $0.00 \mathrm{E}+00$ & $0.00 \mathrm{E}+00$ & $9.49 \mathrm{E}-14$ \\
\hline $\mid-123$ & $5.85 \mathrm{E}-07$ & $8.28 \mathrm{E}-09$ & $5.76 \mathrm{E}-07$ & $5.17 \mathrm{E}-15$ & $5.85 \mathrm{E}-14$ & 8.28E-09 \\
\hline $\mid-124$ & $1.75 \mathrm{E}-05$ & $5.96 \mathrm{E}-08$ & 1.74E-05 & $0.00 \mathrm{E}+00$ & $0.00 \mathrm{E}+00$ & 5.96E-08 \\
\hline $\mid-125$ & $6.73 \mathrm{E}-07$ & $6.06 \mathrm{E}-10$ & $6.72 \mathrm{E}-07$ & $0.00 \mathrm{E}+00$ & $0.00 \mathrm{E}+00$ & $6.06 \mathrm{E}-10$ \\
\hline $\mid-126$ & $1.45 \mathrm{E}-05$ & $2.54 \mathrm{E}-08$ & $1.13 \mathrm{E}-05$ & $1.74 \mathrm{E}-06$ & $1.46 \mathrm{E}-06$ & 1.77E-06 \\
\hline $\mid-128$ & $3.63 \mathrm{E}-09$ & $6.05 \mathrm{E}-10$ & 2.17E-09 & $8.46 \mathrm{E}-10$ & $7.26 \mathrm{E}-12$ & 1.45E-09 \\
\hline $\mid-129$ & 2.97E-05 & $4.51 \mathrm{E}-10$ & $6.22 \mathrm{E}-07$ & $1.50 \mathrm{E}-05$ & $1.41 \mathrm{E}-05$ & $1.50 \mathrm{E}-05$ \\
\hline $\mid-130$ & $6.77 \mathrm{E}-06$ & $1.12 \mathrm{E}-07$ & $6.39 \mathrm{E}-06$ & $2.26 \mathrm{E}-07$ & 4.67E-08 & 3.38E-07 \\
\hline $\mid-131$ & $1.40 \mathrm{E}-05$ & $2.09 \mathrm{E}-08$ & 8.66E-06 & 2.97E-06 & 2.33E-06 & 2.99E-06 \\
\hline $\mid-132$ & 1.17E-06 & $9.16 \mathrm{E}-08$ & 1.05E-06 & $2.48 \mathrm{E}-08$ & $E-09$ & 1.16E-07 \\
\hline $\mid-133$ & $4.21 \mathrm{E}-06$ & $3.24 \mathrm{E}-08$ & $3.21 \mathrm{E}-06$ & 7.42E-07 & 2.27E-07 & 7.74E-07 \\
\hline $\mid-134$ & $3.78 \mathrm{E}-07$ & $6.05 \mathrm{E}-08$ & $3.12 \mathrm{E}-07$ & $5.65 \mathrm{E}-09$ & $E-10$ & $6.62 \mathrm{E}-08$ \\
\hline $\mid-135$ & $3.01 \mathrm{E}-06$ & $8.51 \mathrm{E}-08$ & $2.68 \mathrm{E}-06$ & 2.17E-07 & 2.64E-08 & 3.02E-07 \\
\hline Xe-122 & $1.52 \mathrm{E}-07$ & $5.53 \mathrm{E}-08$ & $9.69 \mathrm{E}-08$ & $0.00 \mathrm{E}+00$ & $0.00 \mathrm{E}+00$ & $3 \mathrm{E}-08$ \\
\hline Xe-123 & $5.45 \mathrm{E}-08$ & $2.47 \mathrm{E}-08$ & $2.98 \mathrm{E}-08$ & $1.39 \mathrm{E}-16$ & $3.02 \mathrm{E}-15$ & $2.47 \mathrm{E}-08$ \\
\hline Xe-125 & $1.37 \mathrm{E}-08$ & $1.34 \mathrm{E}-08$ & $3.64 \mathrm{E}-10$ & $0.00 \mathrm{E}+00$ & $0.00 \mathrm{E}+00$ & 34E-08 \\
\hline Xe-127 & $1.48 \mathrm{E}-08$ & $1.48 \mathrm{E}-08$ & $0.00 \mathrm{E}+00$ & $0.00 \mathrm{E}+00$ & $0.00 \mathrm{E}+00$ & $1.48 \mathrm{E}-08$ \\
\hline $\mathrm{Xe}-129 \mathrm{~m}$ & $1.28 \mathrm{E}-09$ & $1.28 \mathrm{E}-09$ & $0.00 \mathrm{E}+00$ & $0.00 \mathrm{E}+00$ & $0.00 \mathrm{E}+00$ & $.28 \mathrm{E}-09$ \\
\hline $\mathrm{Xe}-131 \mathrm{~m}$ & $4.75 \mathrm{E}-10$ & $4.75 \mathrm{E}-10$ & $0.00 \mathrm{E}+00$ & $0.00 \mathrm{E}+00$ & $0.00 \mathrm{E}+00$ & $4.75 \mathrm{E}-10$ \\
\hline Xe-133 & 1.94E-09 & 1.94E-09 & $0.00 \mathrm{E}+00$ & $0.00 \mathrm{E}+00$ & $0.00 \mathrm{E}+00$ & 1.94E-09 \\
\hline Xe-133m & 1.65E-09 & 1.65E-09 & $0.00 \mathrm{E}+00$ & $0.00 \mathrm{E}+00$ & $0.00 \mathrm{E}+00$ & 1.65E-09 \\
\hline Xe-135 & $1.28 \mathrm{E}-08$ & $1.28 \mathrm{E}-08$ & $0.00 \mathrm{E}+00$ & $1.60 \mathrm{E}-17$ & $7.53 \mathrm{E}-19$ & $1.28 \mathrm{E}-08$ \\
\hline $\mathrm{Xe}-135 \mathrm{~m}$ & 1.40E-09 & 1.40E-09 & $0.00 \mathrm{E}+00$ & $3.14 \mathrm{E}-19$ & $1.48 \mathrm{E}-20$ & 1.40E-09 \\
\hline Xe-138 & $2.74 \mathrm{E}-08$ & $2.38 \mathrm{E}-08$ & 3.59E-09 & $0.00 \mathrm{E}+00$ & $0.00 \mathrm{E}+00$ & $2.38 \mathrm{E}-08$ \\
\hline Cs-126 & $1.38 \mathrm{E}-20$ & $1.28 \mathrm{E}-20$ & $1.02 \mathrm{E}-21$ & $0.00 \mathrm{E}+00$ & $0.00 \mathrm{E}+00$ & $1.28 \mathrm{E}-20$ \\
\hline Cs-129 & 1.27E-07 & $1.42 \mathrm{E}-08$ & $1.13 \mathrm{E}-07$ & $0.00 \mathrm{E}+00$ & $0.00 \mathrm{E}+00$ & $1.42 \mathrm{E}-08$ \\
\hline Cs-131 & $2.29 \mathrm{E}-08$ & $3.94 \mathrm{E}-10$ & $2.25 \mathrm{E}-08$ & $0.00 \mathrm{E}+00$ & $0.00 \mathrm{E}+00$ & $3.94 \mathrm{E}-10$ \\
\hline Cs-132 & $9.08 \mathrm{E}-07$ & 3.99E-08 & $7.43 \mathrm{E}-07$ & $1.20 \mathrm{E}-07$ & $4.53 \mathrm{E}-09$ & $1.60 \mathrm{E}-07$ \\
\hline Cs-134 & 7.37E-06 & $8.94 \mathrm{E}-08$ & $2.29 \mathrm{E}-06$ & 4.77E-06 & $2.24 \mathrm{E}-07$ & $4.86 \mathrm{E}-06$ \\
\hline Cs-134m & $2.34 \mathrm{E}-09$ & $8.79 \mathrm{E}-10$ & 1.24E-09 & $1.84 \mathrm{E}-10$ & $3.43 \mathrm{E}-11$ & 1.06E-09 \\
\hline Cs-135 & $4.41 \mathrm{E}-07$ & $0.00 \mathrm{E}+00$ & $0.00 \mathrm{E}+00$ & $4.21 \mathrm{E}-07$ & 1.99E-08 & $4.21 \mathrm{E}-07$ \\
\hline Cs-136 & $3.51 \mathrm{E}-06$ & $1.25 \mathrm{E}-07$ & $2.61 \mathrm{E}-06$ & $7.46 \mathrm{E}-07$ & $3.14 \mathrm{E}-08$ & 8.71E-07 \\
\hline Cs-137 & 4.46E-06 & $3.23 \mathrm{E}-08$ & $8.42 \mathrm{E}-07$ & $3.43 \mathrm{E}-06$ & $1.62 \mathrm{E}-07$ & $3.46 \mathrm{E}-06$ \\
\hline Cs-138 & $3.75 \mathrm{E}-08$ & $3.21 \mathrm{E}-08$ & 5.37E-09 & $0.00 \mathrm{E}+00$ & $0.00 \mathrm{E}+00$ & $3.21 \mathrm{E}-08$ \\
\hline Ba-131 & $6.22 \mathrm{E}-07$ & $2.55 \mathrm{E}-08$ & 5.97E-07 & $0.00 \mathrm{E}+00$ & $0.00 \mathrm{E}+00$ & $2.55 \mathrm{E}-08$ \\
\hline Ba-133 & $1.36 \mathrm{E}-06$ & $2.05 \mathrm{E}-08$ & $6.09 \mathrm{E}-07$ & $6.95 \mathrm{E}-07$ & $3.28 \mathrm{E}-08$ & 7.16E-07 \\
\hline $\mathrm{Ba}-133 \mathrm{~m}$ & $9.52 \mathrm{E}-08$ & 3.09E-09 & $3.21 \mathrm{E}-08$ & 5.87E-08 & 1.34E-09 & $6.18 \mathrm{E}-08$ \\
\hline $\mathrm{Ba}-135 \mathrm{~m}$ & $2.45 \mathrm{E}-08$ & $2.70 \mathrm{E}-09$ & $2.18 \mathrm{E}-08$ & $0.00 \mathrm{E}+00$ & $0.00 \mathrm{E}+00$ & $2.70 \mathrm{E}-09$ \\
\hline $\mathrm{Ba}-137 \mathrm{~m}$ & $2.55 \mathrm{E}-16$ & $2.37 \mathrm{E}-16$ & $1.84 \mathrm{E}-17$ & $0.00 \mathrm{E}+00$ & $0.00 \mathrm{E}+00$ & 2.37E-16 \\
\hline Ba-139 & $1.27 \mathrm{E}-08$ & 1.12E-09 & $4.70 \mathrm{E}-10$ & $1.11 \mathrm{E}-08$ & $1.55 \mathrm{E}-11$ & $1.22 \mathrm{E}-08$ \\
\hline
\end{tabular}


Table 3. $100-\mathrm{m}$ elevated release, Class $\mathrm{D}, 4 \mathrm{~m} / \mathrm{s}$ wind, no rain, continued.

\begin{tabular}{|c|c|c|c|c|c|c|}
\hline Juclide & $\begin{array}{c}\text { 7-day } \\
\text { Total } \\
(1+2+3+4)\end{array}$ & $\begin{array}{c}\text { Cloud } \\
\text { shine } \\
(1)\end{array}$ & $\begin{array}{c}\text { Ground } \\
\text { shine } \\
(2)\end{array}$ & $\begin{array}{c}\text { Inhalation } \\
\text { (3) }\end{array}$ & $\begin{array}{c}\text { Resus- } \\
\text { pension } \\
(4)\end{array}$ & $\begin{array}{c}\text { Plume } \\
\text { Passage } \\
(1)+(3)\end{array}$ \\
\hline Ba-140 & $2.48 \mathrm{E}-06$ & $1.32 \mathrm{E}-08$ & $2.05 \mathrm{E}-06$ & $3.85 \mathrm{E}-07$ & $2.76 \mathrm{E}-08$ & $3.98 \mathrm{E}-07$ \\
\hline Ba-141 & 4.64E-09 & 3.87E-09 & $7.12 \mathrm{E}-10$ & $4.34 \mathrm{E}-11$ & $1.49 \mathrm{E}-11$ & 3.91E-09 \\
\hline Ba-142 & $1.83 \mathrm{E}-08$ & $1.38 \mathrm{E}-08$ & $4.50 \mathrm{E}-09$ & $0.00 \mathrm{E}+00$ & $0.00 \mathrm{E}+00$ & $38 \mathrm{E}-08$ \\
\hline La-137 & $2.35 \mathrm{E}-06$ & $0.00 \mathrm{E}+00$ & $0.00 \mathrm{E}+00$ & $2.24 \mathrm{E}-06$ & 1.06E-07 & $2.24 \mathrm{E}-06$ \\
\hline La-138 & $3.72 \mathrm{E}-05$ & $0.00 \mathrm{E}+00$ & $0.00 \mathrm{E}+00$ & $3.55 \mathrm{E}-05$ & $1.68 \mathrm{E}-06$ & $55 \mathrm{E}-05$ \\
\hline La-140 & $1.68 \mathrm{E}-06$ & $1.34 \mathrm{E}-07$ & $1.00 \mathrm{E}-06$ & $5.30 \mathrm{E}-07$ & $1.23 \mathrm{E}-08$ & 6.64E-07 \\
\hline La-141 & $5.30 \mathrm{E}-09$ & $2.02 \mathrm{E}-09$ & $2.24 \mathrm{E}-09$ & $8.47 \mathrm{E}-10$ & $1.94 \mathrm{E}-10$ & 2.87E-09 \\
\hline La-142 & $1.38 \mathrm{E}-07$ & $1.04 \mathrm{E}-07$ & $3.44 \mathrm{E}-08$ & $0.00 \mathrm{E}+00$ & $0.00 \mathrm{E}+00$ & 1.04E-07 \\
\hline Ce-139 & $1.16 \mathrm{E}-06$ & 8.34E-09 & $2.51 \mathrm{E}-07$ & $8.59 \mathrm{E}-07$ & $4.01 \mathrm{E}-08$ & 8.67E-07 \\
\hline Ce-141 & $1.08 \mathrm{E}-06$ & 4.29E-09 & $1.18 \mathrm{E}-07$ & $9.13 \mathrm{E}-07$ & 4.11E-08 & $9.17 \mathrm{E}-07$ \\
\hline Ce-143 & 1.27E-07 & $1.43 \mathrm{E}-08$ & $1.13 \mathrm{E}-07$ & $0.00 \mathrm{E}+00$ & $0.00 \mathrm{E}+00$ & $1.43 \mathrm{E}-08$ \\
\hline Ce-144 & 3.92E-05 & $2.88 \mathrm{E}-09$ & 7.43E-08 & 3.74E-05 & $1.75 \mathrm{E}-06$ & 3.74E-05 \\
\hline $\mathrm{Pr}-142$ & $3.49 \mathrm{E}-07$ & $3.32 \mathrm{E}-09$ & $1.20 \mathrm{E}-08$ & $3.29 \mathrm{E}-07$ & 4.7 & 3.32E-07 \\
\hline Pr-143 & $1.16 \mathrm{E}-14$ & $5.14 \mathrm{E}-16$ & $1.11 \mathrm{E}-14$ & $0.00 \mathrm{E}+00$ & $0.00 \mathrm{E}+00$ & $5.14 \mathrm{E}-16$ \\
\hline Pr-144 & $1.39 \mathrm{E}-10$ & $1.24 \mathrm{E}-10$ & $1.48 \mathrm{E}-11$ & $0.00 \mathrm{E}+00$ & $=00$ & $1.24 \mathrm{E}-10$ \\
\hline Pr-144m & $9.78 \mathrm{E}-11$ & $8.73 \mathrm{E}-11$ & 1.05E-11 & $0.00 \mathrm{E}+00$ & $0.00 \mathrm{E}+00$ & $8.73 \mathrm{E}-11$ \\
\hline Nd-141 & $9.70 \mathrm{E}-10$ & $0.00 \mathrm{E}+00$ & $0.00 \mathrm{E}+00$ & $9.67 \mathrm{E}-10$ & $2.38 \mathrm{E}-12$ & $.67 \mathrm{E}-10$ \\
\hline Nd-147 & $9.11 \mathrm{E}-07$ & 7.39E-09 & $1.75 \mathrm{E}-07$ & 7.00E-07 & $2.91 \mathrm{E}-08$ & 7.07E-07 \\
\hline $\mathrm{Nd}-149$ & $4.48 \mathrm{E}-08$ & $1.34 \mathrm{E}-08$ & 6.96E-09 & $2.41 \mathrm{E}-08$ & $E-10$ & $3.75 \mathrm{E}-08$ \\
\hline $\mathrm{Pm}-143$ & 1.54E-06 & $1.72 \mathrm{E}-08$ & 4.54E-07 & 1.02E-06 & 4.79E-08 & 1.04E-06 \\
\hline $\mathrm{Pm}-144$ & $2.38 \mathrm{E}-06$ & $8.78 \mathrm{E}-08$ & $2.30 \mathrm{E}-06$ & $0.00 \mathrm{E}+00$ & $0.00 \mathrm{E}+00$ & 8.78E-08 \\
\hline $\mathrm{Pm}-145$ & $2.61 \mathrm{E}-06$ & $9.19 \mathrm{E}-10$ & $4.18 \mathrm{E}-08$ & $2.45 \mathrm{E}-06$ & 1.16E-07 & $2.45 \mathrm{E}-06$ \\
\hline $\mathrm{Pm}-146$ & $1.63 \mathrm{E}-05$ & $4.23 \mathrm{E}-08$ & $1.12 \mathrm{E}-06$ & $1.44 \mathrm{E}-05$ & $6.79 \mathrm{E}-07$ & $1.44 \mathrm{E}-05$ \\
\hline $\mathrm{Pm}-147$ & 4.14E-06 & $2.03 E-13$ & $5.92 \mathrm{E}-12$ & 3.96E-06 & 1.86E-07 & 3.96E-06 \\
\hline $\mathrm{Pm}-148$ & $5.46 \mathrm{E}-07$ & $3.29 \mathrm{E}-08$ & $5.14 \mathrm{E}-07$ & $0.00 \mathrm{E}+00$ & $0.00 \mathrm{E}+00$ & $3.29 \mathrm{E}-08$ \\
\hline $\mathrm{Pm}-148 \mathrm{~m}$ & $2.93 \mathrm{E}-06$ & 1.14E-07 & $2.81 \mathrm{E}-06$ & $0.00 \mathrm{E}+00$ & $0.00 \mathrm{E}+00$ & $1.14 \mathrm{E}-07$ \\
\hline $\mathrm{Pm}-149$ & 3.31E-07 & $6.44 \mathrm{E}-10$ & $7.29 \mathrm{E}-09$ & $3.14 \mathrm{E}-07$ & $8.35 \mathrm{E}-09$ & $3.15 \mathrm{E}-07$ \\
\hline Pm-151 & $1.42 \mathrm{E}-07$ & $1.82 \mathrm{E}-08$ & $1.23 \mathrm{E}-07$ & 3.07E-12 & $\mathrm{E}-12$ & $1.82 \mathrm{E}-08$ \\
\hline Sm-146 & 8.77E-03 & $0.00 \mathrm{E}+00$ & $0.00 \mathrm{E}+00$ & $8.37 \mathrm{E}-03$ & 3.95E-04 & 8.37E-03 \\
\hline Sm-147 & 7.96E-03 & $0.00 \mathrm{E}+00$ & $0.00 \mathrm{E}+00$ & $7.60 \mathrm{E}-03$ & $3.58 \mathrm{E}-04$ & 7.60E-03 \\
\hline Sm-151 & $3.21 \mathrm{E}-06$ & $5.11 \mathrm{E}-14$ & $7.60 \mathrm{E}-12$ & 3.07E-06 & 1.45E-07 & 3.07E-06 \\
\hline Sm-153 & $2.54 \mathrm{E}-07$ & $2.80 \mathrm{E}-09$ & $3.72 \mathrm{E}-08$ & $2.09 \mathrm{E}-07$ & $5.22 \mathrm{E}-09$ & $2.12 \mathrm{E}-07$ \\
\hline Eu-150b & $2.76 \mathrm{E}-05$ & $0.00 \mathrm{E}+00$ & $0.00 \mathrm{E}+00$ & $2.64 \mathrm{E}-05$ & $1.24 \mathrm{E}-06$ & 2.64E-05 \\
\hline Eu-152 & $2.42 \mathrm{E}-05$ & $6.57 \mathrm{E}-08$ & $1.62 \mathrm{E}-06$ & $2.15 \mathrm{E}-05$ & $1.01 \mathrm{E}-06$ & $2.16 \mathrm{E}-05$ \\
\hline Eu-152m & $1.20 \mathrm{E}-07$ & 1.67E-08 & $3.50 \mathrm{E}-08$ & $6.79 \mathrm{E}-08$ & $5.55 \mathrm{E}-10$ & 8.46E-08 \\
\hline Eu-154 & $3.15 \mathrm{E}-05$ & $7.25 \mathrm{E}-08$ & $1.75 \mathrm{E}-06$ & $2.84 \mathrm{E}-05$ & $1.34 \mathrm{E}-06$ & $2.85 \mathrm{E}-05$ \\
\hline Eu-155 & 1.06E-07 & $3.17 \mathrm{E}-09$ & 1.03E-07 & $0.00 \mathrm{E}+00$ & $0.00 \mathrm{E}+00$ & 3.17E-09 \\
\hline Eu-156 & $1.64 \mathrm{E}-06$ & $8.17 \mathrm{E}-08$ & 1.56E-06 & $0.00 \mathrm{E}+00$ & $0.00 \mathrm{E}+00$ & 8.17E-08 \\
\hline Gd-148 & $9.26 \mathrm{E}-03$ & $0.00 \mathrm{E}+00$ & $0.00 \mathrm{E}+00$ & $8.84 \mathrm{E}-03$ & 4.17E-04 & 8.84E-03 \\
\hline Gd-152 & $6.92 \mathrm{E}-03$ & $0.00 \mathrm{E}+00$ & $0.00 \mathrm{E}+00$ & $6.61 \mathrm{E}-03$ & $3.12 \mathrm{E}-04$ & $6.61 \mathrm{E}-03$ \\
\hline Gd-153 & 1.86E-07 & 4.93E-09 & $1.81 \mathrm{E}-07$ & $0.00 \mathrm{E}+00$ & $0.00 \mathrm{E}+00$ & 4.93E-09 \\
\hline
\end{tabular}


Table 3. $100-\mathrm{m}$ elevated release, Class $\mathrm{D}, 4 \mathrm{~m} / \mathrm{s}$ wind, no rain, continued.

\begin{tabular}{|c|c|c|c|c|c|c|}
\hline de & $\begin{array}{c}\text { 7-day } \\
\text { Total } \\
(1+2+3+4)\end{array}$ & $\begin{array}{l}\text { Cloud } \\
\text { shine } \\
(1)\end{array}$ & $\begin{array}{c}\text { Ground } \\
\text { shine } \\
(2)\end{array}$ & $\begin{array}{c}\text { Inhalation } \\
(3)\end{array}$ & $\begin{array}{c}\text { Resus- } \\
\text { pension } \\
(4)\end{array}$ & $\begin{array}{c}\text { Plume } \\
\text { Passage } \\
(1)+(3)\end{array}$ \\
\hline Gd-159 & $1.28 \mathrm{E}-07$ & $2.01 \mathrm{E}-09$ & 9.79E-09 & 1.15E-07 & 1.61E-09 & 1.17E-07 \\
\hline Tb-157 & $6.83 \mathrm{E}-07$ & $1.42 \mathrm{E}-10$ & $6.73 \mathrm{E}-09$ & $6.46 \mathrm{E}-07$ & 04E-08 & $46 \mathrm{E}-07$ \\
\hline Tb-158 & $2.40 \mathrm{E}-05$ & $0.00 \mathrm{E}+00$ & $0.00 \mathrm{E}+00$ & $2.29 \mathrm{E}-05$ & 08E-06 & $.29 \mathrm{E}-05$ \\
\hline Tb-160 & 3.90E-06 & $6.26 \mathrm{E}-08$ & $1.48 \mathrm{E}-06$ & $2.25 \mathrm{E}-06$ & $4 E-07$ & $.31 \mathrm{E}-06$ \\
\hline Tb-161 & $3.65 \mathrm{E}-07$ & $0.00 \mathrm{E}+00$ & $0.00 \mathrm{E}+00$ & $3.51 \mathrm{E}-07$ & $34 \mathrm{E}-08$ & $3.51 \mathrm{E}-07$ \\
\hline Dy-157 & $.35 \mathrm{E}-08$ & $1.71 \mathrm{E}-08$ & 3.63E-08 & $3.74 \mathrm{E}-13$ & 61E-13 & $1.71 \mathrm{E}-08$ \\
\hline Dy-159 & $2.10 \mathrm{E}-07$ & $0.00 \mathrm{E}+00$ & $0.00 \mathrm{E}+00$ & 2.01E-07 & 38E-09 & $2.01 \mathrm{E}-07$ \\
\hline Dy-165 & 1.63E-09 & $9.78 \mathrm{E}-10$ & $6.52 \mathrm{E}-10$ & $0.00 \mathrm{E}+00$ & $00 \mathrm{E}+00$ & $9.78 \mathrm{E}-10$ \\
\hline Dy-166 & 3.91E-06 & 1.79E-09 & $5.28 \mathrm{E}-08$ & 2.27E-06 & 58E-06 & $2.27 \mathrm{E}-06$ \\
\hline Ho-164 & $3.27 \mathrm{E}-10$ & $0.00 \mathrm{E}+00$ & $0.00 \mathrm{E}+00$ & $3.27 \mathrm{E}-10$ & $63 \mathrm{E}-13$ & $3.27 \mathrm{E}-10$ \\
\hline Ho-164m & 1.60E-09 & $0.00 \mathrm{E}+00$ & $0.00 \mathrm{E}+00$ & 1.60E-09 & $23 \mathrm{E}-12$ & OEE-09 \\
\hline Ho-166 & 7.69E-05 & $1.52 \mathrm{E}-09$ & 9.26E-09 & $7.55 \mathrm{E}-05$ & 1.37E-06 & $7.55 \mathrm{E}-05$ \\
\hline Ho-166m & 2.49E-06 & 9.16E-08 & $2.40 \mathrm{E}-06$ & $0.00 \mathrm{E}+00$ & $0.00 \mathrm{E}+00$ & $.16 \mathrm{E}-08$ \\
\hline Er-169 & 2.23E-07 & $1.06 \mathrm{E}-13$ & $5.63 \mathrm{E}-12$ & $2.14 \mathrm{E}-07$ & 8.60E-09 & $2.14 \mathrm{E}-07$ \\
\hline Er-171 & 1.22E-07 & $1.85 \mathrm{E}-08$ & $3.62 \mathrm{E}-08$ & 6.67E-08 & E-10 & $.52 \mathrm{E}-08$ \\
\hline Tm-170 & $2.79 \mathrm{E}-06$ & $2.58 \mathrm{E}-10$ & 8.87E-09 & $2.65 \mathrm{E}-06$ & $1.24 \mathrm{E}-07$ & $2.65 \mathrm{E}-06$ \\
\hline Tm-171 & $9.75 \mathrm{E}-07$ & $2.91 \mathrm{E}-11$ & $1.14 \mathrm{E}-09$ & $9.30 \mathrm{E}-07$ & $37 \mathrm{E}-08$ & $.30 \mathrm{E}-07$ \\
\hline Yb-169 & 95E-07 & $1.59 \mathrm{E}-08$ & $4.79 \mathrm{E}-07$ & $0.00 \mathrm{E}+00$ & $0.00 \mathrm{E}+00$ & $1.59 \mathrm{E}-08$ \\
\hline Yb-175 & 3.87E-08 & 2.17E-09 & $3.66 \mathrm{E}-08$ & $0.00 \mathrm{E}+00$ & $0.00 \mathrm{E}+00$ & 2.17E-09 \\
\hline Lu-174 & $2 E-06$ & $0.00 \mathrm{E}+00$ & $0.00 \mathrm{E}+00$ & $3.84 \mathrm{E}-06$ & 81E-07 & $3.84 \mathrm{E}-06$ \\
\hline Lu-174m & $2.54 \mathrm{E}-06$ & $0.00 \mathrm{E}+00$ & $0.00 \mathrm{E}+00$ & $2.43 \mathrm{E}-06$ & E-07 & $2.43 \mathrm{E}-06$ \\
\hline Lu-176 & 7.07E-05 & $E+00$ & $0.00 \mathrm{E}+00$ & $6.76 \mathrm{E}-05$ & E-06 & $6.76 \mathrm{E}-05$ \\
\hline Lu-176m & $3.38 \mathrm{E}-08$ & $0.00 \mathrm{E}+00$ & $0.00 \mathrm{E}+00$ & 3.37E-08 & E-10 & $3.37 \mathrm{E}-08$ \\
\hline Lu-177 & 20E-07 & E-09 & $.09 \mathrm{E}-08$ & $\mathrm{E}-07$ & E-08 & E-07 \\
\hline Lu-177m & $5.25 \mathrm{E}-06$ & $5.51 \mathrm{E}-08$ & $1.57 \mathrm{E}-06$ & $3.46 \mathrm{E}-06$ & E-07 & $3.52 \mathrm{E}-06$ \\
\hline Lu-178 & 5E-09 & $E+00$ & $00 \mathrm{E}+00$ & $=-09$ & -13 & E-09 \\
\hline Lu-178m & 7.71E-10 & $0.00 \mathrm{E}+00$ & $0.00 \mathrm{E}+00$ & $7.71 \mathrm{E}-10$ & $=-13$ & $7.71 \mathrm{E}-10$ \\
\hline $\mathrm{Hf}-175$ & $3.65 \mathrm{E}-07$ & $E+00$ & $0.00 \mathrm{E}+00$ & $\mathrm{E}-07$ & -08 & $.48 \mathrm{E}-07$ \\
\hline $\mathrm{Hf}-177 \mathrm{~m}$ & $.00 \mathrm{E}-09$ & $0.00 \mathrm{E}+00$ & $0.00 \mathrm{E}+00$ & 5.00E-09 & $\mathrm{E}-12$ & $5.00 \mathrm{E}-09$ \\
\hline $\mathrm{Hf}-178 \mathrm{~m}$ & 62E-05 & $0.00 \mathrm{E}+00$ & $0.00 \mathrm{E}+00$ & E-05 & $=-06$ & 36E-05 \\
\hline $\mathrm{Hf}-179 \mathrm{~m}$ & $1.01 \mathrm{E}-06$ & $0.00 \mathrm{E}+00$ & $0.00 \mathrm{E}+00$ & $9.69 \mathrm{E}-07$ & 4.30E-08 & $9.69 \mathrm{E}-07$ \\
\hline Hf-181 & $2.04 \mathrm{E}-06$ & $3.03 \mathrm{E}-08$ & 7.94E-07 & 1.16E-06 & 5.27E-08 & 1.19E-06 \\
\hline Hf-182 & $9.15 \mathrm{E}-05$ & $2.16 \mathrm{E}-11$ & $3.74 \mathrm{E}-08$ & $8.73 \mathrm{E}-05$ & $4.12 \mathrm{E}-06$ & $8.73 \mathrm{E}-05$ \\
\hline Hf-183 & 03E-08 & $0.00 \mathrm{E}+00$ & $0.00 \mathrm{E}+00$ & 1.01E-08 & 1.62E-10 & $1.01 \mathrm{E}-08$ \\
\hline Ta-179 & 5.23E-07 & $0.00 \mathrm{E}+00$ & $0.00 \mathrm{E}+00$ & $4.99 \mathrm{E}-07$ & $2.35 \mathrm{E}-08$ & 4.99E-07 \\
\hline Ta-180m & 8.72E-09 & $0.00 \mathrm{E}+00$ & $0.00 \mathrm{E}+00$ & 8.65E-09 & $6.28 \mathrm{E}-11$ & 8.65E-09 \\
\hline Тa-182 & $6.40 \mathrm{E}-06$ & $7.45 \mathrm{E}-08$ & $1.75 \mathrm{E}-06$ & $4.38 \mathrm{E}-06$ & $2.04 \mathrm{E}-07$ & $4.45 \mathrm{E}-06$ \\
\hline Ta-182m & $6.90 \mathrm{E}-10$ & $6.78 \mathrm{E}-12$ & $1.67 \mathrm{E}-10$ & $4.97 \mathrm{E}-10$ & $1.95 \mathrm{E}-11$ & $5.04 \mathrm{E}-10$ \\
\hline Ta-183 & $5.14 \mathrm{E}-07$ & $0.00 \mathrm{E}+00$ & $0.00 \mathrm{E}+00$ & 4.97E-07 & 1.77E-08 & 4.97E-07 \\
\hline Ta-184 & 1.12E-07 & $0.00 \mathrm{E}+00$ & $0.00 \mathrm{E}+00$ & $1.11 \mathrm{E}-07$ & $8.55 \mathrm{E}-10$ & $1.11 \mathrm{E}-07$ \\
\hline Та-185 & 5.76E-09 & 4.37E-16 & $1.98 \mathrm{E}-14$ & $5.76 \mathrm{E}-09$ & $6.56 \mathrm{E}-12$ & $5.76 \mathrm{E}-09$ \\
\hline
\end{tabular}


Table 3. $100-\mathrm{m}$ elevated release, Class $\mathrm{D}, 4 \mathrm{~m} / \mathrm{s}$ wind, no rain, continued.

\begin{tabular}{|c|c|c|c|c|c|c|}
\hline uclide & $\begin{array}{c}\text { 7-day } \\
\text { Total } \\
(1+2+3+4)\end{array}$ & $\begin{array}{l}\text { Cloud } \\
\text { shine } \\
(1)\end{array}$ & $\begin{array}{l}\text { Ground } \\
\text { shine } \\
(2)\end{array}$ & $\begin{array}{c}\text { Inhalation } \\
(3)\end{array}$ & $\begin{array}{c}\text { Resus- } \\
\text { pension } \\
(4)\end{array}$ & $\begin{array}{c}\text { Plume } \\
\text { Passage } \\
(1)+(3)\end{array}$ \\
\hline Ta-186 & $4.20 \mathrm{E}-11$ & $0.00 \mathrm{E}+00$ & $0.00 \mathrm{E}+00$ & $4.20 \mathrm{E}-11$ & $7.60 \mathrm{E}-15$ & $4.20 \mathrm{E}-11$ \\
\hline W-179 & $1.19 \mathrm{E}-10$ & $0.00 \mathrm{E}+00$ & $0.00 \mathrm{E}+00$ & $1.18 \mathrm{E}-10$ & $9.83 \mathrm{E}-13$ & $1.18 \mathrm{E}-10$ \\
\hline W-181 & 7.98E-08 & $1.81 \mathrm{E}-09$ & $6.59 \mathrm{E}-08$ & $1.15 \mathrm{E}-08$ & $5.36 \mathrm{E}-10$ & $33 \mathrm{E}-08$ \\
\hline W-185 & 8.92E-08 & $1.54 \mathrm{E}-12$ & 4.37E-11 & $8.52 \mathrm{E}-08$ & 3.93E-09 & $8.52 \mathrm{E}-08$ \\
\hline W-187 & 2.33E-07 & 2.61E-08 & $1.44 \mathrm{E}-07$ & $6.14 \mathrm{E}-08$ & 1.03E-09 & $5 \mathrm{E}-08$ \\
\hline W-188 & 5.99E-07 & $2.51 \mathrm{E}-10$ & 7.78E-08 & 4.91E-07 & $3.00 \mathrm{E}-08$ & 4.91E-07 \\
\hline Re-182b & $1.25 \mathrm{E}-06$ & 9.83E-08 & $1.15 \mathrm{E}-06$ & $0.00 \mathrm{E}+00$ & $0.00 \mathrm{E}+00$ & 83E-08 \\
\hline $\mathrm{Re}-182 \mathrm{a}$ & $2.40 \mathrm{E}-07$ & $6.46 \mathrm{E}-08$ & $1.76 \mathrm{E}-07$ & $0.00 \mathrm{E}+00$ & $0.00 \mathrm{E}+00$ & $6.46 \mathrm{E}-08$ \\
\hline Re-184 & $1.81 \mathrm{E}-06$ & $5.12 \mathrm{E}-08$ & $1.24 \mathrm{E}-06$ & $4.98 \mathrm{E}-07$ & $2.25 \mathrm{E}-08$ & $5.49 \mathrm{E}-07$ \\
\hline e-184m & $1.60 \mathrm{E}-06$ & $2.19 \mathrm{E}-08$ & $6.47 \mathrm{E}-07$ & 8.87E-07 & $4.21 \mathrm{E}-08$ & $9.09 \mathrm{E}-07$ \\
\hline$e-186$ & 3.45E-07 & 1.12E-09 & $1.95 \mathrm{E}-08$ & 3.14E-07 & $\mathrm{E}-08$ & $5 \mathrm{E}-07$ \\
\hline e-186m & $3.00 \mathrm{E}-06$ & $9.93 \mathrm{E}-12$ & 1.53E-08 & $2.84 \mathrm{E}-06$ & 1.39E-07 & 2.84E-06 \\
\hline e-187 & 5.69E-09 & $0.00 \mathrm{E}+00$ & $0.00 \mathrm{E}+00$ & 5.43E-09 & $2.56 \mathrm{E}-10$ & $5.43 \mathrm{E}-09$ \\
\hline e-188 & $2.41 \mathrm{E}-07$ & 3.16E-09 & $1.28 \mathrm{E}-08$ & $2.22 \mathrm{E}-07$ & 2.93E-09 & $2.25 \mathrm{E}-07$ \\
\hline$e-188 m$ & 4.51E-09 & $5.41 \mathrm{E}-11$ & $2.38 \mathrm{E}-10$ & 16E-09 & -11 & $1 \mathrm{E}-09$ \\
\hline Re-189 & $1.26 \mathrm{E}-07$ & $0.00 \mathrm{E}+00$ & $0.00 \mathrm{E}+00$ & $1.24 \mathrm{E}-07$ & 2.12E-09 & $1.24 \mathrm{E}-07$ \\
\hline Os-185 & 1.07E-06 & 4.01E-08 & $.03 \mathrm{E}-06$ & $0.00 \mathrm{E}+00$ & $0.00 \mathrm{E}+00$ & $1 \mathrm{E}-08$ \\
\hline Os-189m & 3.13E-09 & $0.00 \mathrm{E}+00$ & $0.00 \mathrm{E}+00$ & 3.11E-09 & $1.73 \mathrm{E}-11$ & $3.11 \mathrm{E}-09$ \\
\hline Os-190m & $7.89 \mathrm{E}-10$ & $7.05 \mathrm{E}-10$ & $44 \mathrm{E}-11$ & $0.00 \mathrm{E}+00$ & $0.00 \mathrm{E}+00$ & $.05 \mathrm{E}-10$ \\
\hline Os-191 & 5.14E-07 & 3.83E-09 & $1.05 \mathrm{E}-07$ & $3.88 \mathrm{E}-07$ & 1.66E-08 & $3.92 \mathrm{E}-07$ \\
\hline Os-191m & $5.80 \mathrm{E}-09$ & $2.23 \mathrm{E}-10$ & 4.31E-09 & $8.15 \mathrm{E}-10$ & $4.62 \mathrm{E}-10$ & 1.04E-09 \\
\hline Os-193 & 01E-08 & 3.62E-09 & $2.65 \mathrm{E}-08$ & $0.00 \mathrm{E}+00$ & $0.00 \mathrm{E}+00$ & $3.62 \mathrm{E}-09$ \\
\hline Os-194 & 8.69E-05 & $2.11 \mathrm{E}-10$ & $1.15 \mathrm{E}-07$ & $8.29 \mathrm{E}-05$ & 3.91E-06 & $8.29 \mathrm{E}-05$ \\
\hline $\mid r-190$ & $.38 \mathrm{E}-06$ & $7.85 \mathrm{E}-08$ & 1.76E-06 & $5.21 \mathrm{E}-07$ & $2.17 \mathrm{E}-08$ & $6.00 \mathrm{E}-07$ \\
\hline Ir-190m & 1.18E-08 & 1.35E-09 & 7.79E-09 & 2.54E-09 & $9.05 \mathrm{E}-11$ & $3.89 \mathrm{E}-09$ \\
\hline Ir-190n & 16E-09 & $5.19 \mathrm{E}-11$ & $1.02 \mathrm{E}-09$ & $7.91 \mathrm{E}-11$ & $1.12 \mathrm{E}-11$ & $1.31 \mathrm{E}-10$ \\
\hline Ir-192 & 4.23E-06 & 4.59E-08 & $1.23 \mathrm{E}-06$ & $2.82 \mathrm{E}-06$ & 1.30E-07 & 2.87E-06 \\
\hline Ir-192m & 96E-05 & 2.06E-11 & $4.10 \mathrm{E}-08$ & 5.69E-05 & $2.68 \mathrm{E}-06$ & $5.69 \mathrm{E}-05$ \\
\hline Ir-194 & $.61 \mathrm{E}-07$ & 4.96E-09 & $2.20 \mathrm{E}-08$ & $3.29 \mathrm{E}-07$ & 4.75E-09 & $3.34 \mathrm{E}-07$ \\
\hline Ir-194m & 9.46E-06 & $1.32 \mathrm{E}-07$ & $3.50 \mathrm{E}-06$ & 5.57E-06 & $2.60 \mathrm{E}-07$ & $5.70 \mathrm{E}-06$ \\
\hline Pt-191 & 2.34E-07 & $1.55 \mathrm{E}-08$ & $2.18 \mathrm{E}-07$ & $0.00 \mathrm{E}+00$ & $0.00 \mathrm{E}+00$ & $1.55 \mathrm{E}-08$ \\
\hline Pt-193 & $6.08 \mathrm{E}-09$ & $2.46 \mathrm{E}-12$ & $8.16 \mathrm{E}-10$ & 5.02E-09 & $2.37 \mathrm{E}-10$ & $5.02 \mathrm{E}-09$ \\
\hline Pt-193m & 4.85E-08 & $5.36 \mathrm{E}-10$ & $1.14 \mathrm{E}-08$ & 3.54E-08 & $1.21 \mathrm{E}-09$ & $3.59 \mathrm{E}-08$ \\
\hline Pt-195m & 7.42E-08 & 3.60E-09 & 7.06E-08 & $0.00 \mathrm{E}+00$ & $0.00 \mathrm{E}+00$ & $3.60 \mathrm{E}-09$ \\
\hline Pt-197 & 5.98E-08 & 1.21E-09 & $6.10 \mathrm{E}-09$ & $5.17 \mathrm{E}-08$ & $7.22 \mathrm{E}-10$ & $5.29 \mathrm{E}-08$ \\
\hline Pt-197m & 1.36E-08 & 2.63E-09 & 1.83E-09 & 9.04E-09 & $7.25 \mathrm{E}-11$ & 1.17E-08 \\
\hline Au-194 & $6.37 \mathrm{E}-07$ & $6.21 \mathrm{E}-08$ & 4.84E-07 & $8.92 \mathrm{E}-08$ & $2.04 \mathrm{E}-09$ & $1.51 \mathrm{E}-07$ \\
\hline Au-195 & $1.45 \mathrm{E}-07$ & 4.23E-09 & 1.40E-07 & $0.00 \mathrm{E}+00$ & $0.00 \mathrm{E}+00$ & $4.23 \mathrm{E}-09$ \\
\hline Au-195m & $2.79 \mathrm{E}-13$ & $8.16 \mathrm{E}-15$ & $2.71 \mathrm{E}-13$ & $0.00 \mathrm{E}+00$ & $0.00 \mathrm{E}+00$ & $8.16 \mathrm{E}-15$ \\
\hline Au-198 & 7.65E-07 & $2.23 \mathrm{E}-08$ & 2.87E-07 & 4.43E-07 & $1.28 \mathrm{E}-08$ & $4.65 \mathrm{E}-07$ \\
\hline Au-198m & $7.20 \mathrm{E}-07$ & $2.76 \mathrm{E}-10$ & 1.71E-07 & 5.30E-07 & 1.92E-08 & $5.30 \mathrm{E}-07$ \\
\hline
\end{tabular}


Table 3. $100-\mathrm{m}$ elevated release, Class $\mathrm{D}, 4 \mathrm{~m} / \mathrm{s}$ wind, no rain, continued.

\begin{tabular}{|c|c|c|c|c|c|c|}
\hline ide & $\begin{array}{c}\text { 7-day } \\
\text { Total } \\
(1+2+3+4)\end{array}$ & $\begin{array}{c}\text { Cloud } \\
\text { shine } \\
(1)\end{array}$ & $\begin{array}{l}\text { Ground } \\
\text { shine } \\
(2)\end{array}$ & $\begin{array}{c}\text { Inhalation } \\
(3)\end{array}$ & $\begin{array}{c}\text { Resus- } \\
\text { pension } \\
(4)\end{array}$ & $\begin{array}{c}\text { Plume } \\
\text { Passage } \\
(1)+(3)\end{array}$ \\
\hline Au-199 & $7.98 \mathrm{E}-08$ & 4.99E-09 & $7.48 \mathrm{E}-08$ & $0.00 \mathrm{E}+00$ & $0.00 \mathrm{E}+00$ & 4.99E-09 \\
\hline $\mathrm{Hg}-194$ & $5.18 \mathrm{E}-06$ & 1.27E-09 & $1.04 \mathrm{E}-06$ & $3.96 \mathrm{E}-06$ & $1.89 \mathrm{E}-07$ & $3.96 \mathrm{E}-06$ \\
\hline $\mathrm{Hg}-197$ & $1.24 \mathrm{E}-07$ & $3.45 \mathrm{E}-09$ & $5.33 \mathrm{E}-08$ & $6.51 \mathrm{E}-08$ & $1.88 \mathrm{E}-09$ & $6.86 \mathrm{E}-08$ \\
\hline $\mathrm{Hg}-197 \mathrm{~m}$ & 1.65E-07 & 4.93E-09 & $4.71 \mathrm{E}-08$ & 1.11E-07 & $29 \mathrm{E}-09$ & 1.16E-07 \\
\hline $\mathrm{Hg}-199 \mathrm{~m}$ & 1.83E-09 & $0.00 \mathrm{E}+00$ & $0.00 \mathrm{E}+00$ & 1.83E-09 & $1.33 \mathrm{E}-12$ & $1.83 \mathrm{E}-09$ \\
\hline $\mathrm{Hg}-203$ & 9.92E-07 & $1.28 \mathrm{E}-08$ & $3.46 \mathrm{E}-07$ & 6.06E-07 & $2.76 \mathrm{E}-08$ & $6.19 \mathrm{E}-07$ \\
\hline TI-200 & 4.85E-07 & $7.29 \mathrm{E}-08$ & $4.12 \mathrm{E}-07$ & $0.00 \mathrm{E}+00$ & $0.00 \mathrm{E}+00$ & $7.29 \mathrm{E}-08$ \\
\hline TI-201 & $8.19 \mathrm{E}-08$ & 4.79E-09 & 7.71E-08 & $0.00 \mathrm{E}+00$ & $0.00 \mathrm{E}+00$ & 4.79E-09 \\
\hline TI-202 & $7.25 \mathrm{E}-07$ & $2.57 \mathrm{E}-08$ & 5.97E-07 & $9.85 \mathrm{E}-08$ & 4.11E-09 & $1.24 \mathrm{E}-07$ \\
\hline TI-204 & 2.82E-07 & $5.66 \mathrm{E}-11$ & 1.87E-09 & 2.67E-07 & $.26 \mathrm{E}-08$ & 2.67E-07 \\
\hline TI-206 & $9.40 \mathrm{E}-15$ & $0.00 \mathrm{E}+00$ & $0.00 \mathrm{E}+00$ & $9.40 \mathrm{E}-15$ & $6.83 \mathrm{E}-19$ & $9.40 \mathrm{E}-15$ \\
\hline TI-207 & $5.96 \mathrm{E}-15$ & $5.46 \mathrm{E}-15$ & $4.98 \mathrm{E}-16$ & $0.00 \mathrm{E}+00$ & $0.00 \mathrm{E}+00$ & $5.46 \mathrm{E}-15$ \\
\hline TI-208 & $3.86 \mathrm{E}-14$ & $3.64 \mathrm{E}-14$ & $2.18 \mathrm{E}-15$ & $0.00 \mathrm{E}+00$ & $0.00 \mathrm{E}+00$ & $3.64 \mathrm{E}-14$ \\
\hline TI-209 & $8.78 \mathrm{E}-11$ & $4.23 \mathrm{E}-17$ & $2.92 \mathrm{E}-18$ & $8.75 \mathrm{E}-11$ & $2.78 \mathrm{E}-13$ & $8.75 \mathrm{E}-11$ \\
\hline $\mathrm{Pb}-202$ & $1.21 \mathrm{E}-05$ & $7.00 \mathrm{E}-11$ & 1.27E-07 & $1.15 \mathrm{E}-05$ & $=-07$ & $1.15 \mathrm{E}-05$ \\
\hline $\mathrm{Pb}-203$ & $2.49 \mathrm{E}-07$ & $1.64 \mathrm{E}-08$ & $1.94 \mathrm{E}-07$ & $3.79 \mathrm{E}-08$ & $9.99 \mathrm{E}-10$ & 5.43E-08 \\
\hline $\mathrm{Pb}-205$ & $2.96 \mathrm{E}-07$ & $3.20 \mathrm{E}-12$ & 1.10E-09 & $2.81 \mathrm{E}-07$ & $1.33 \mathrm{E}-08$ & $2.81 \mathrm{E}-07$ \\
\hline Pb-209 & 7.70E-09 & $0.00 \mathrm{E}+00$ & $0.00 \mathrm{E}+00$ & 7.68E-09 & $2.44 \mathrm{E}-11$ & 7.68E-09 \\
\hline $\mathrm{Pb}-210$ & $1.48 \mathrm{E}-03$ & $7.36 \mathrm{E}-11$ & 4.31E-09 & $1.41 \mathrm{E}-03$ & $6.68 \mathrm{E}-05$ & $1.41 \mathrm{E}-03$ \\
\hline $\mathrm{Pb}-211$ & 1.90E-09 & $1.56 \mathrm{E}-09$ & $3.44 \mathrm{E}-10$ & $0.00 \mathrm{E}+00$ & $0.00 \mathrm{E}+00$ & 1.56E-09 \\
\hline $\mathrm{Pb}-212$ & 2.43E-07 & $5.31 \mathrm{E}-08$ & 1.90E-07 & $0.00 \mathrm{E}+00$ & $0.00 \mathrm{E}+00$ & 5.31E-08 \\
\hline $\mathrm{Pb}-214$ & $3.69 \mathrm{E}-08$ & $2.94 \mathrm{E}-08$ & 5.35E-09 & 1.96E-09 & $1.52 \mathrm{E}-10$ & $3.14 \mathrm{E}-08$ \\
\hline $\mathrm{Bi}-206$ & $3.46 \mathrm{E}-06$ & 1.90E-07 & $3.26 \mathrm{E}-06$ & $0.00 \mathrm{E}+00$ & $0.00 \mathrm{E}+00$ & 1.90E-07 \\
\hline $\mathrm{Bi}-207$ & 4.44E-06 & $8.88 \mathrm{E}-08$ & $2.21 \mathrm{E}-06$ & 2.04E-06 & $9.63 \mathrm{E}-08$ & 2.13E-06 \\
\hline $\mathrm{Bi}-210$ & $2.11 \mathrm{E}-05$ & $1.19 \mathrm{E}-16$ & $1.62 \mathrm{E}-13$ & $2.00 \mathrm{E}-05$ & $1.08 \mathrm{E}-06$ & $2.00 \mathrm{E}-05$ \\
\hline $\mathrm{Bi}-210 \mathrm{~m}$ & 8.19E-04 & $0.00 \mathrm{E}+00$ & $0.00 \mathrm{E}+00$ & 7.82E-04 & $3.69 \mathrm{E}-05$ & 7.82E-04 \\
\hline $\mathrm{Bi}-211$ & $4.84 \mathrm{E}-15$ & $4.43 \mathrm{E}-15$ & $4.04 \mathrm{E}-16$ & $0.00 \mathrm{E}+00$ & $0.00 \mathrm{E}+00$ & $4.43 \mathrm{E}-15$ \\
\hline $\mathrm{Bi}-212$ & $5.29 \mathrm{E}-08$ & $4.27 \mathrm{E}-08$ & 1.02E-08 & $0.00 \mathrm{E}+00$ & $0.00 \mathrm{E}+00$ & 4.27E-08 \\
\hline $\mathrm{Bi}-213$ & $5.93 \mathrm{E}-09$ & $3.70 \mathrm{E}-09$ & $9.24 \mathrm{E}-10$ & $1.29 \mathrm{E}-09$ & $6.63 \mathrm{E}-12$ & 4.99E-09 \\
\hline $\mathrm{Bi}-214$ & 15E-08 & $8.12 \mathrm{E}-09$ & $1.08 \mathrm{E}-09$ & $2.18 \mathrm{E}-09$ & $1.13 \mathrm{E}-10$ & 1.03E-08 \\
\hline Po-210 & $9.29 \mathrm{E}-04$ & $4.95 \mathrm{E}-13$ & $1.23 \mathrm{E}-11$ & $8.88 \mathrm{E}-04$ & $4.14 \mathrm{E}-05$ & 8.88E-04 \\
\hline Po-211 & $0.00 \mathrm{E}+00$ & $0.00 \mathrm{E}+00$ & $00 \mathrm{E}+00$ & $0.00 \mathrm{E}+00$ & $0.00 \mathrm{E}+00$ & $0.00 \mathrm{E}+00$ \\
\hline Po-213 & $2.75 \mathrm{E}-18$ & $0.00 \mathrm{E}+00$ & $0.00 \mathrm{E}+00$ & $2.75 \mathrm{E}-18$ & $0.00 \mathrm{E}+00$ & $2.75 \mathrm{E}-18$ \\
\hline Po-214 & $3.37 \mathrm{E}-16$ & $1.72 \mathrm{E}-23$ & $1.01 \mathrm{E}-21$ & $3.29 \mathrm{E}-16$ & $7.53 \mathrm{E}-18$ & $3.29 \mathrm{E}-16$ \\
\hline Po-215 & $1.54 \mathrm{E}-15$ & $1.28 \mathrm{E}-15$ & $2.60 \mathrm{E}-16$ & $0.00 \mathrm{E}+00$ & $0.00 \mathrm{E}+00$ & $1.28 \mathrm{E}-15$ \\
\hline Po-216 & $9.49 \mathrm{E}-13$ & $2.08 \mathrm{E}-13$ & $7.41 \mathrm{E}-13$ & $0.00 \mathrm{E}+00$ & $0.00 \mathrm{E}+00$ & $2.08 \mathrm{E}-13$ \\
\hline Po-218 & $4.48 \mathrm{E}-09$ & $3.59 \mathrm{E}-09$ & $6.62 \mathrm{E}-10$ & $2.10 \mathrm{E}-10$ & $1.72 \mathrm{E}-11$ & 3.80E-09 \\
\hline At-211 & $8.47 \mathrm{E}-08$ & $2.03 E-09$ & $8.27 \mathrm{E}-08$ & $1.93 \mathrm{E}-12$ & $1.32 \mathrm{E}-11$ & 2.03E-09 \\
\hline At-217 & $6.97 \mathrm{E}-14$ & $4.37 \mathrm{E}-14$ & $1.07 \mathrm{E}-14$ & $1.53 \mathrm{E}-14$ & $7.81 \mathrm{E}-17$ & $5.90 \mathrm{E}-14$ \\
\hline Rn-218 & 7.34E-14 & $3.66 \mathrm{E}-21$ & $2.14 \mathrm{E}-19$ & $7.01 \mathrm{E}-14$ & $3.31 \mathrm{E}-15$ & $7.01 \mathrm{E}-14$ \\
\hline Rn-219 & $3.48 \mathrm{E}-12$ & $2.85 \mathrm{E}-12$ & $6.30 \mathrm{E}-13$ & $0.00 \mathrm{E}+00$ & $0.00 \mathrm{E}+00$ & $2.85 \mathrm{E}-12$ \\
\hline
\end{tabular}


Table 3. $100-\mathrm{m}$ elevated release, Class $\mathrm{D}, 4 \mathrm{~m} / \mathrm{s}$ wind, no rain, continued.

\begin{tabular}{|c|c|c|c|c|c|c|}
\hline Juclide & $\begin{array}{c}\text { 7-day } \\
\text { Total } \\
(1+2+3+4)\end{array}$ & $\begin{array}{c}\text { Cloud } \\
\text { shine } \\
(1)\end{array}$ & $\begin{array}{l}\text { Ground } \\
\text { shine } \\
(2)\end{array}$ & $\begin{array}{c}\text { Inhalation } \\
(3)\end{array}$ & $\begin{array}{c}\text { Resus- } \\
\text { pension } \\
(4)\end{array}$ & $\begin{array}{c}\text { Plume } \\
\text { Passage } \\
(1)+(3)\end{array}$ \\
\hline Rn-220 & $3.51 \mathrm{E}-10$ & $7.62 \mathrm{E}-11$ & $2.75 \mathrm{E}-10$ & $0.00 \mathrm{E}+00$ & $0.00 \mathrm{E}+00$ & $7.62 \mathrm{E}-11$ \\
\hline Rn-222 & $8.10 \mathrm{E}-08$ & $6.29 \mathrm{E}-08$ & $1.64 \mathrm{E}-08$ & $1.41 \mathrm{E}-09$ & $2.68 \mathrm{E}-10$ & $6.43 \mathrm{E}-08$ \\
\hline Fr-221 & $6.74 \mathrm{E}-10$ & $4.35 \mathrm{E}-10$ & $1.09 \mathrm{E}-10$ & $1.29 \mathrm{E}-10$ & $7.06 \mathrm{E}-13$ & $5.64 \mathrm{E}-10$ \\
\hline Fr-223 & $1.04 \mathrm{E}-06$ & $3.01 \mathrm{E}-10$ & $5.61 \mathrm{E}-10$ & 9.92E-07 & $4.61 \mathrm{E}-08$ & 9.92E-07 \\
\hline Ra-222 & $7.97 \mathrm{E}-11$ & $3.97 \mathrm{E}-18$ & $2.33 \mathrm{E}-16$ & $7.61 \mathrm{E}-11$ & $3.61 \mathrm{E}-12$ & 7.61E-11 \\
\hline Ra-223 & 8.77E-04 & 1.47E-08 & $3.81 \mathrm{E}-07$ & $8.42 \mathrm{E}-04$ & $3.48 \mathrm{E}-05$ & 8.42E-04 \\
\hline Ra-224 & $3.78 \mathrm{E}-04$ & $2.94 \mathrm{E}-09$ & $1.10 \mathrm{E}-06$ & $3.65 \mathrm{E}-04$ & $1.18 \mathrm{E}-05$ & $3.65 \mathrm{E}-04$ \\
\hline Ra-225 & $8.31 \mathrm{E}-04$ & $3.91 \mathrm{E}-10$ & $8.29 \mathrm{E}-08$ & 7.92E-04 & $3.90 \mathrm{E}-05$ & 7.92E-04 \\
\hline Ra-226 & 7.53E-03 & $6.32 \mathrm{E}-10$ & $1.05 \mathrm{E}-06$ & 7.19E-03 & 3.39E-04 & 7.19E-03 \\
\hline Ra-228 & $1.83 \mathrm{E}-03$ & $6.58 \mathrm{E}-09$ & $1.26 \mathrm{E}-06$ & 1.74E-03 & $8.57 \mathrm{E}-05$ & 1.74E-03 \\
\hline Ac-225 & $9.14 \mathrm{E}-04$ & 8.80E-09 & $2.75 \mathrm{E}-07$ & $8.78 \mathrm{E}-04$ & $3.57 \mathrm{E}-05$ & 8.78E-04 \\
\hline Ac-227 & 1.38E-01 & $4.90 \mathrm{E}-11$ & $2.89 \mathrm{E}-08$ & 1.31E-01 & $6.20 \mathrm{E}-03$ & 1.31E-01 \\
\hline Ac-228 & $1.35 \mathrm{E}-05$ & 4.73E-08 & $6.49 \mathrm{E}-08$ & $1.28 \mathrm{E}-05$ & 5.99E-07 & $1.28 \mathrm{E}-05$ \\
\hline Th-226 & 3.35E-09 & $2.15 \mathrm{E}-10$ & $4.57 \mathrm{E}-11$ & 2.91E-09 & $1.75 \mathrm{E}-10$ & 3.13E-09 \\
\hline Th-227 & $1.73 \mathrm{E}-03$ & 5.86E-09 & $2.30 \mathrm{E}-07$ & $1.65 \mathrm{E}-03$ & 7.63E-05 & $1.65 \mathrm{E}-03$ \\
\hline Th-228 & $3.63 \mathrm{E}-02$ & $1.18 \mathrm{E}-10$ & $1.04 \mathrm{E}-07$ & 3.47E-02 & 1.64E-03 & 3.47E-02 \\
\hline Th-229 & $1.84 \mathrm{E}-01$ & 4.79E-09 & $1.62 \mathrm{E}-07$ & $1.76 \mathrm{E}-01$ & $8.30 \mathrm{E}-03$ & 1.76E-01 \\
\hline Th-230 & $2.78 \mathrm{E}-02$ & $2.17 \mathrm{E}-11$ & 1.32E-09 & $2.66 \mathrm{E}-02$ & $1.25 \mathrm{E}-03$ & $2.66 \mathrm{E}-02$ \\
\hline Th-231 & 9.93E-08 & $6.25 \mathrm{E}-10$ & 5.93E-09 & $9.09 \mathrm{E}-08$ & 1.83E-09 & $9.15 \mathrm{E}-08$ \\
\hline Th-232 & $1.23 \mathrm{E}-01$ & $1.04 \mathrm{E}-11$ & $2.36 \mathrm{E}-09$ & 1.17E-01 & $5.53 \mathrm{E}-03$ & 1.17E-01 \\
\hline Th-234 & $3.76 \mathrm{E}-06$ & 1.13E-09 & 3.62E-08 & $3.56 \mathrm{E}-06$ & $1.58 \mathrm{E}-07$ & 3.56E-06 \\
\hline Pa-230 & 8.87E-07 & $3.79 \mathrm{E}-08$ & 8.49E-07 & $2.85 \mathrm{E}-11$ & $5.99 \mathrm{E}-11$ & $3.79 \mathrm{E}-08$ \\
\hline Pa-231 & $9.13 \mathrm{E}-02$ & $1.68 \mathrm{E}-09$ & $5.22 \mathrm{E}-08$ & $8.72 \mathrm{E}-02$ & $4.11 \mathrm{E}-03$ & 8.72E-02 \\
\hline Pa-232 & 7.46E-06 & $1.85 \mathrm{E}-17$ & $5.65 \mathrm{E}-14$ & 7.23E-06 & E-07 & 7.23E-06 \\
\hline Pa-233 & $1.30 \mathrm{E}-06$ & 1.19E-08 & $3.14 \mathrm{E}-07$ & $9.35 \mathrm{E}-07$ & 4.17E-08 & 9.47E-07 \\
\hline Pa-234 & $2.54 \mathrm{E}-07$ & $1.00 \mathrm{E}-07$ & $1.54 \mathrm{E}-07$ & $4.73 \mathrm{E}-12$ & $1.75 \mathrm{E}-12$ & $1.00 \mathrm{E}-07$ \\
\hline Pa-234m & $1.09 \mathrm{E}-12$ & $3.81 \mathrm{E}-13$ & $5.83 \mathrm{E}-13$ & $1.22 \mathrm{E}-13$ & $5.77 \mathrm{E}-15$ & $5.03 \mathrm{E}-13$ \\
\hline U-230 & 4.43E-08 & $8.48 \mathrm{E}-10$ & $2.86 \mathrm{E}-08$ & 2.77E-09 & $1.21 \mathrm{E}-08$ & 3.62E-09 \\
\hline U-231 & 7.94E-08 & $3.80 \mathrm{E}-09$ & $7.45 \mathrm{E}-08$ & $4.03 \mathrm{E}-10$ & $6.89 \mathrm{E}-10$ & $4.20 \mathrm{E}-09$ \\
\hline U-232 & 7.09E-02 & $1.48 \mathrm{E}-11$ & 1.76E-09 & $6.77 \mathrm{E}-02$ & $3.19 \mathrm{E}-03$ & $6.77 \mathrm{E}-02$ \\
\hline U-233 & $1.43 \mathrm{E}-02$ & 1.33E-11 & $7.28 \mathrm{E}-10$ & 1.37E-02 & $6.45 \mathrm{E}-04$ & 1.37E-02 \\
\hline U-234 & $1.41 \mathrm{E}-02$ & $8.50 \mathrm{E}-12$ & $1.18 \mathrm{E}-09$ & $1.34 \mathrm{E}-02$ & $6.34 \mathrm{E}-04$ & 1.34E-02 \\
\hline U-235 & $1.29 \mathrm{E}-02$ & 8.59E-09 & $2.70 \mathrm{E}-07$ & $1.23 \mathrm{E}-02$ & 5.81E-04 & $1.23 \mathrm{E}-02$ \\
\hline U-236 & $1.33 \mathrm{E}-02$ & $6.72 \mathrm{E}-12$ & 1.07E-09 & $1.27 \mathrm{E}-02$ & $5.98 \mathrm{E}-04$ & $1.27 \mathrm{E}-02$ \\
\hline U-237 & $1.74 \mathrm{E}-07$ & 7.43E-09 & $1.66 \mathrm{E}-07$ & $2.16 \mathrm{E}-12$ & $4.10 \mathrm{E}-12$ & 7.43E-09 \\
\hline U-238 & $1.25 \mathrm{E}-02$ & $7.28 \mathrm{E}-12$ & 4.69E-09 & $1.20 \mathrm{E}-02$ & $5.64 \mathrm{E}-04$ & $1.20 \mathrm{E}-02$ \\
\hline U-239 & $3.29 \mathrm{E}-09$ & $3.93 \mathrm{E}-10$ & $8.87 \mathrm{E}-10$ & 1.96E-09 & $4.18 \mathrm{E}-11$ & 2.35E-09 \\
\hline U-240 & 4.33E-07 & 1.79E-08 & $5.82 \mathrm{E}-08$ & $3.53 \mathrm{E}-07$ & $4.34 \mathrm{E}-09$ & 3.71E-07 \\
\hline $\mathrm{Np}-235$ & 6.99E-09 & $1.12 \mathrm{E}-10$ & $6.87 \mathrm{E}-09$ & $4.99 \mathrm{E}-15$ & $1.12 \mathrm{E}-14$ & $1.12 \mathrm{E}-10$ \\
\hline Np-236a & $1.08 \mathrm{E}-02$ & $7.29 \mathrm{E}-09$ & $2.30 \mathrm{E}-07$ & 1.03E-02 & $4.85 \mathrm{E}-04$ & 1.03E-02 \\
\hline Vp-236b & 4.23E-07 & 2.76E-09 & $1.63 \mathrm{E}-08$ & $2.12 \mathrm{E}-07$ & $1.92 \mathrm{E}-07$ & 2.15E-07 \\
\hline
\end{tabular}


Table 3. $100-\mathrm{m}$ elevated release, Class $\mathrm{D}, 4 \mathrm{~m} / \mathrm{s}$ wind, no rain, continued.

\begin{tabular}{|c|c|c|c|c|c|c|}
\hline Vuclide & $\begin{array}{c}\text { 7-day } \\
\text { Total } \\
(1+2+3+4)\end{array}$ & $\begin{array}{c}\text { Cloud } \\
\text { shine } \\
(1)\end{array}$ & $\begin{array}{c}\text { Ground } \\
\text { shine } \\
(2)\end{array}$ & $\begin{array}{c}\text { Inhalation } \\
(3)\end{array}$ & $\begin{array}{c}\text { Resus- } \\
\text { pension } \\
(4)\end{array}$ & $\begin{array}{c}\text { Plume } \\
\text { Passage } \\
(1)+(3)\end{array}$ \\
\hline $\mathrm{Np}-237$ & 5.33E-02 & 1.29E-09 & 7.63E-08 & $5.09 \mathrm{E}-02$ & $2.40 \mathrm{E}-03$ & 5.09E-02 \\
\hline Np-238 & $4.15 \mathrm{E}-07$ & $3.18 \mathrm{E}-08$ & $3.05 \mathrm{E}-07$ & $3.28 \mathrm{E}-08$ & $4.52 \mathrm{E}-08$ & $6.46 \mathrm{E}-08$ \\
\hline Np-239 & $3.44 \mathrm{E}-07$ & $9.22 \mathrm{E}-09$ & 1.17E-07 & $2.11 \mathrm{E}-07$ & 5.96E-09 & $2.20 \mathrm{E}-07$ \\
\hline $\mathrm{Np}-240$ & $4.22 \mathrm{E}-08$ & 3.17E-08 & $1.01 \mathrm{E}-08$ & $3.39 \mathrm{E}-10$ & $3.03 \mathrm{E}-11$ & $3.20 \mathrm{E}-08$ \\
\hline Np-240m & $1.09 \mathrm{E}-10$ & $2.89 \mathrm{E}-11$ & $3.02 \mathrm{E}-12$ & $7.38 \mathrm{E}-11$ & $3.49 \mathrm{E}-12$ & $1.03 \mathrm{E}-10$ \\
\hline Pu-236 & 1.47E-02 & $6.64 \mathrm{E}-12$ & 1.45E-09 & $1.41 \mathrm{E}-02$ & $6.63 \mathrm{E}-04$ & 1.41E-02 \\
\hline Pu-237 & $8.45 \mathrm{E}-08$ & 2.66E-09 & $8.18 \mathrm{E}-08$ & $2.17 \mathrm{E}-12$ & $4.77 \mathrm{E}-12$ & 2.66E-09 \\
\hline Pu-238 & $3.33 \mathrm{E}-02$ & 4.93E-12 & $1.25 \mathrm{E}-09$ & $3.18 \mathrm{E}-02$ & $1.50 \mathrm{E}-03$ & $3.18 \mathrm{E}-02$ \\
\hline Pu-239 & $3.60 \mathrm{E}-02$ & $4.57 \mathrm{E}-12$ & $5.53 \mathrm{E}-10$ & $3.44 \mathrm{E}-02$ & $1.62 \mathrm{E}-03$ & 3.44E-02 \\
\hline Pu-240 & $3.60 \mathrm{E}-02$ & $4.83 \mathrm{E}-12$ & $1.20 \mathrm{E}-09$ & $3.44 \mathrm{E}-02$ & $1.62 \mathrm{E}-03$ & 3.44E-02 \\
\hline$P u-241$ & $6.09 \mathrm{E}-04$ & $1.12 \mathrm{E}-15$ & $2.32 \mathrm{E}-12$ & $5.82 \mathrm{E}-04$ & $2.74 \mathrm{E}-05$ & 5.82E-04 \\
\hline$P u-242$ & $3.42 \mathrm{E}-02$ & $4.10 \mathrm{E}-12$ & $9.97 \mathrm{E}-10$ & $3.27 \mathrm{E}-02$ & $1.54 \mathrm{E}-03$ & $3.27 \mathrm{E}-02$ \\
\hline$P u-243$ & $3.51 \mathrm{E}-09$ & 1.10E-09 & 1.61E-09 & $6.16 \mathrm{E}-10$ & $1.79 \mathrm{E}-10$ & 1.72E-09 \\
\hline Pu-244 & $3.24 \mathrm{E}-02$ & $8.85 \mathrm{E}-10$ & 4.34E-07 & 3.09E-02 & 1.46E-03 & 3.09E-02 \\
\hline Pu-245 & $8.25 \mathrm{E}-08$ & $2.26 \mathrm{E}-08$ & $5.95 \mathrm{E}-08$ & $1.00 \mathrm{E}-10$ & $2.91 \mathrm{E}-10$ & $2.27 \mathrm{E}-08$ \\
\hline Pu-246 & $1.29 \mathrm{E}-06$ & $4.55 \mathrm{E}-08$ & $1.24 \mathrm{E}-06$ & $4.48 \mathrm{E}-10$ & $2.10 \mathrm{E}-09$ & 4.59E-08 \\
\hline Am-241 & 5.67E-02 & 1.05E-09 & $4.30 \mathrm{E}-08$ & $5.42 \mathrm{E}-02$ & E-03 & $5.42 \mathrm{E}-02$ \\
\hline Am-242 & $6.44 \mathrm{E}-06$ & $7.30 \mathrm{E}-10$ & $3.55 \mathrm{E}-09$ & $6.14 \mathrm{E}-06$ & $2.88 \mathrm{E}-07$ & $6.14 \mathrm{E}-06$ \\
\hline$A m-242 m$ & $5.48 \mathrm{E}-02$ & $6.60 \mathrm{E}-11$ & $2.92 \mathrm{E}-08$ & $5.23 \mathrm{E}-02$ & $2.47 \mathrm{E}-03$ & $5.23 \mathrm{E}-02$ \\
\hline Am-243 & $5.66 \mathrm{E}-02$ & $2.95 \mathrm{E}-09$ & $2.57 \mathrm{E}-07$ & $5.40 \mathrm{E}-02$ & $2.55 \mathrm{E}-03$ & $5.40 \mathrm{E}-02$ \\
\hline Am-244 & $3.53 \mathrm{E}-07$ & $4.27 \mathrm{E}-08$ & $9.93 \mathrm{E}-08$ & $1.39 \mathrm{E}-07$ & $7.15 \mathrm{E}-08$ & $1.82 \mathrm{E}-07$ \\
\hline $\mathrm{Am}-245$ & 2.47E-09 & $1.18 \mathrm{E}-09$ & $7.26 \mathrm{E}-10$ & $4.95 \mathrm{E}-10$ & $7.02 \mathrm{E}-11$ & $1.68 \mathrm{E}-09$ \\
\hline $\mathrm{Am}-246$ & $2.08 \mathrm{E}-08$ & 1.66E-08 & 3.47E-09 & $6.09 \mathrm{E}-10$ & $4.04 \mathrm{E}-11$ & $1.72 \mathrm{E}-08$ \\
\hline $\mathrm{Cm}-242$ & $1.90 \mathrm{E}-03$ & $5.49 \mathrm{E}-12$ & 1.35E-09 & $1.81 \mathrm{E}-03$ & $8.48 \mathrm{E}-05$ & $1.81 \mathrm{E}-03$ \\
\hline$m-243$ & $3.79 \mathrm{E}-02$ & 7.10E-09 & $2.12 \mathrm{E}-07$ & $3.62 \mathrm{E}-02$ & $1.71 \mathrm{E}-03$ & $3.62 \mathrm{E}-02$ \\
\hline $\mathrm{Cm}-244$ & $3.01 \mathrm{E}-02$ & $4.67 \mathrm{E}-12$ & $1.21 \mathrm{E}-09$ & $2.88 \mathrm{E}-02$ & 1.36E-03 & $2.88 \mathrm{E}-02$ \\
\hline $\mathrm{Cm}-245$ & $5.84 \mathrm{E}-02$ & 3.96E-09 & $1.24 \mathrm{E}-07$ & $5.58 \mathrm{E}-02$ & $2.63 \mathrm{E}-03$ & $5.58 \mathrm{E}-02$ \\
\hline $\mathrm{Cm}-246$ & $5.75 \mathrm{E}-02$ & $3.93 \mathrm{E}-12$ & 1.07E-09 & $5.49 \mathrm{E}-02$ & $2.59 \mathrm{E}-03$ & $5.49 \mathrm{E}-02$ \\
\hline $\mathrm{Cm}-247$ & 5.49E-07 & 1.79E-08 & $5.30 \mathrm{E}-07$ & $5.10 \mathrm{E}-11$ & 1.33E-09 & $1.80 \mathrm{E}-08$ \\
\hline $\mathrm{Cm}-248$ & $4.98 \mathrm{E}-02$ & $3.44 \mathrm{E}-12$ & $8.60 \mathrm{E}-10$ & $4.76 \mathrm{E}-02$ & $2.24 \mathrm{E}-03$ & 4.76E-02 \\
\hline $\mathrm{Cm}-249$ & $6.69 \mathrm{E}-10$ & $5.06 \mathrm{E}-10$ & $1.64 \mathrm{E}-10$ & $4.68 \mathrm{E}-20$ & $3.96 \mathrm{E}-18$ & $5.06 \mathrm{E}-10$ \\
\hline $\mathrm{Cm}-250$ & 2.47E-07 & 1.62E-09 & $2.46 \mathrm{E}-07$ & $1.25 \mathrm{E}-13$ & $6.27 \mathrm{E}-11$ & 1.62E-09 \\
\hline Bk-249 & 1.05E-11 & 1.30E-14 & 1.05E-11 & $1.57 \mathrm{E}-15$ & $3.19 \mathrm{E}-14$ & $1.46 \mathrm{E}-14$ \\
\hline Bk-250 & $7.05 \mathrm{E}-08$ & $4.05 \mathrm{E}-08$ & $3.01 \mathrm{E}-08$ & $3.39 \mathrm{E}-15$ & $6.17 \mathrm{E}-14$ & 4.05E-08 \\
\hline Cf-248 & $4.72 \mathrm{E}-07$ & $3.76 \mathrm{E}-12$ & $9.77 \mathrm{E}-10$ & $1.45 \mathrm{E}-07$ & $3.26 \mathrm{E}-07$ & $1.45 \mathrm{E}-07$ \\
\hline Cf-249 & 5.34E-07 & $1.84 \mathrm{E}-08$ & $5.13 \mathrm{E}-07$ & $5.98 \mathrm{E}-10$ & 1.35E-09 & $1.90 \mathrm{E}-08$ \\
\hline Cf-250 & 4.45E-09 & $4.80 \mathrm{E}-12$ & 1.00E-09 & 1.06E-09 & 2.39E-09 & 1.06E-09 \\
\hline Cf-251 & $2.04 \mathrm{E}-07$ & $6.55 \mathrm{E}-09$ & 1.97E-07 & $1.94 \mathrm{E}-21$ & $1.25 \mathrm{E}-19$ & $6.55 \mathrm{E}-09$ \\
\hline Cf-252 & $9.69 \mathrm{E}-10$ & $4.19 \mathrm{E}-12$ & $9.25 \mathrm{E}-10$ & $1.24 \mathrm{E}-11$ & $2.80 \mathrm{E}-11$ & $1.66 \mathrm{E}-11$ \\
\hline 253 & $1.98 \mathrm{E}-10$ & $1.83 \mathrm{E}-12$ & $1.96 \mathrm{E}-10$ & $4.30 \mathrm{E}-23$ & $4.99 \mathrm{E}-18$ & $1.83 \mathrm{E}-12$ \\
\hline Cf-254 & $3.37 \mathrm{E}-14$ & $7.09 \mathrm{E}-16$ & $3.30 \mathrm{E}-14$ & $0.00 \mathrm{E}+00$ & 1.97E-20 & 7.09E-16 \\
\hline
\end{tabular}


Table 3. $100-\mathrm{m}$ elevated release, Class $\mathrm{D}, 4 \mathrm{~m} / \mathrm{s}$ wind, no rain, continued.

\begin{tabular}{|c|c|c|c|c|c|c|}
\hline Nuclide & $\begin{array}{c}\text { 7-day } \\
\text { Total } \\
(1+2+3+4)\end{array}$ & $\begin{array}{l}\text { Cloud } \\
\text { shine } \\
(1)\end{array}$ & $\begin{array}{c}\text { Ground } \\
\text { shine } \\
(2)\end{array}$ & $\begin{array}{c}\text { Inhalation } \\
(3)\end{array}$ & \begin{tabular}{|c|} 
Resus- \\
pension \\
$(4)$ \\
\end{tabular} & $\begin{array}{c}\text { Plume } \\
\text { Passage } \\
(1)+(3)\end{array}$ \\
\hline Es-253 & $9.49 \mathrm{E}-10$ & $1.87 \mathrm{E}-11$ & $9.30 \mathrm{E}-10$ & $5.61 \mathrm{E}-20$ & $1.11 \mathrm{E}-16$ & $1.87 \mathrm{E}-11$ \\
\hline Es-254 & $1.24 \mathrm{E}-06$ & 1.16E-08 & $1.23 \mathrm{E}-06$ & $2.86 \mathrm{E}-16$ & $5.73 \mathrm{E}-13$ & 1.16E-08 \\
\hline Es-254m & $2.99 \mathrm{E}-07$ & $3.14 \mathrm{E}-08$ & $2.67 \mathrm{E}-07$ & $2.79 \mathrm{E}-16$ & 3.38E-13 & $3.14 \mathrm{E}-08$ \\
\hline Fm-254 & $3.08 \mathrm{E}-11$ & $4.87 \mathrm{E}-12$ & $2.59 \mathrm{E}-11$ & $3.40 \mathrm{E}-15$ & $6.20 \mathrm{E}-14$ & $4.87 \mathrm{E}-12$ \\
\hline Fm-255 & 1.90E-09 & $1.13 \mathrm{E}-10$ & 1.79E-09 & $9.39 \mathrm{E}-28$ & $2.11 \mathrm{E}-25$ & $1.13 \mathrm{E}-10$ \\
\hline \multicolumn{3}{|c|}{$\begin{array}{l}\text { NUMBER OF NUCLIDES } \\
\text { EVALUATED: }\end{array}$} & & & & \\
\hline
\end{tabular}


Table 4. Early dose results (Sv/TBq) for a ground-level release at $1 \mathrm{~km}$ downwind distance using stability class $F, 1 \mathrm{~m} / \mathrm{s}$ wind speed, no rain, and $\mathrm{P}-\mathrm{G}$ dispersion parameters (sigmas).

\begin{tabular}{|c|c|c|c|c|c|c|}
\hline Nuclide & $\begin{array}{c}\text { 7-day } \\
\text { Total } \\
(1+2+3+4)\end{array}$ & $\begin{array}{c}\text { Cloud } \\
\text { shine } \\
(1)\end{array}$ & $\begin{array}{c}\text { Ground } \\
\text { shine } \\
(2)\end{array}$ & $\begin{array}{c}\text { Inhalation } \\
(3)\end{array}$ & $\begin{array}{c}\text { Resus- } \\
\text { pension } \\
(4)\end{array}$ & $\begin{array}{c}\text { Plume } \\
\text { Passage } \\
(1)+(3)\end{array}$ \\
\hline $\mathrm{H}-3$ & $8.55 \mathrm{E}-07$ & $0.00 \mathrm{E}+00$ & $0.00 \mathrm{E}+00$ & $4.96 \mathrm{E}-07$ & $3.59 \mathrm{E}-07$ & $4.96 \mathrm{E}-07$ \\
\hline $\mathrm{Be}-7$ & 1.24E-05 & $1.05 \mathrm{E}-07$ & $8.24 \mathrm{E}-06$ & $3.88 \mathrm{E}-06$ & $1.78 \mathrm{E}-07$ & $3.99 \mathrm{E}-06$ \\
\hline $\mathrm{Be}-10$ & $3.46 \mathrm{E}-03$ & $0.00 \mathrm{E}+00$ & $0.00 \mathrm{E}+00$ & $3.30 \mathrm{E}-03$ & 1.56E-04 & $3.30 \mathrm{E}-03$ \\
\hline C-11 & 1.69E-07 & 1.69E-07 & $0.00 \mathrm{E}+00$ & $0.00 \mathrm{E}+00$ & $0.00 \mathrm{E}+00$ & 1.69E-07 \\
\hline C-14 & 3.63E-07 & $0.00 \mathrm{E}+00$ & $0.00 \mathrm{E}+00$ & $3.63 \mathrm{E}-07$ & $0.00 \mathrm{E}+00$ & $3.63 \mathrm{E}-07$ \\
\hline $\mathrm{N}-13$ & $1.24 \mathrm{E}-08$ & $1.11 \mathrm{E}-08$ & $0.00 \mathrm{E}+00$ & $1.28 \mathrm{E}-09$ & $0.00 \mathrm{E}+00$ & $1.24 \mathrm{E}-08$ \\
\hline $0-15$ & $1.08 \mathrm{E}-17$ & $1.08 \mathrm{E}-17$ & $0.00 \mathrm{E}+00$ & $0.00 \mathrm{E}+00$ & $0.00 \mathrm{E}+00$ & $1.08 \mathrm{E}-17$ \\
\hline $\mathrm{N}-16$ & $0.00 \mathrm{E}+00$ & $0.00 \mathrm{E}+00$ & $0.00 \mathrm{E}+00$ & $0.00 \mathrm{E}+00$ & $0.00 \mathrm{E}+00$ & $0.00 \mathrm{E}+00$ \\
\hline F-18 & 1.42E-05 & 5.64E-07 & 1.33E-05 & $2.85 \mathrm{E}-07$ & 8.03E-09 & $8.49 \mathrm{E}-07$ \\
\hline $\mathrm{Na}-22$ & $4.50 \mathrm{E}-04$ & 4.69E-06 & $3.49 \mathrm{E}-04$ & $9.18 \mathrm{E}-05$ & 4.32E-06 & 9.65E-05 \\
\hline $\mathrm{Na}-24$ & 9.66E-05 & $9.48 \mathrm{E}-06$ & $7.38 \mathrm{E}-05$ & 1.31E-05 & $1.58 \mathrm{E}-07$ & $2.26 \mathrm{E}-05$ \\
\hline $\mathrm{Mg}-27$ & 1.06E-08 & $7.15 \mathrm{E}-09$ & 2.33E-09 & 1.15E-09 & $1.88 \mathrm{E}-13$ & 8.30E-09 \\
\hline $\mathrm{Mg}-28$ & $1.58 \mathrm{E}-04$ & $6.84 \mathrm{E}-06$ & 8.35E-05 & 6.67E-05 & 1.02E-06 & 7.35E-05 \\
\hline Al-26 & $1.35 \mathrm{E}-03$ & $6.13 \mathrm{E}-06$ & $4.17 \mathrm{E}-04$ & 8.84E-04 & 4.17E-05 & 8.90E-04 \\
\hline Al-28 & $2.54 \mathrm{E}-16$ & $2.08 \mathrm{E}-16$ & $3.93 \mathrm{E}-17$ & $6.89 \mathrm{E}-18$ & $0.00 \mathrm{E}+00$ & $2.15 \mathrm{E}-16$ \\
\hline Si-31 & $2.71 \mathrm{E}-06$ & 1.37E-09 & 2.43E-09 & $2.70 \mathrm{E}-06$ & 6.99E-09 & $2.70 \mathrm{E}-06$ \\
\hline Si-32 & $6.38 \mathrm{E}-04$ & $0.00 \mathrm{E}+00$ & $0.00 \mathrm{E}+00$ & $6.09 \mathrm{E}-04$ & $2.90 \mathrm{E}-05$ & $6.09 \mathrm{E}-04$ \\
\hline S-37 & $5.42 \mathrm{E}-12$ & $0.00 \mathrm{E}+00$ & $0.00 \mathrm{E}+00$ & $5.42 \mathrm{E}-12$ & $4.73 \mathrm{E}-16$ & $5.42 \mathrm{E}-12$ \\
\hline P-32 & $6.19 \mathrm{E}-05$ & $0.00 \mathrm{E}+00$ & $0.00 \mathrm{E}+00$ & 5.94E-05 & $2.52 \mathrm{E}-06$ & 5.94E-05 \\
\hline P-33 & $6.58 \mathrm{E}-06$ & $0.00 \mathrm{E}+00$ & $0.00 \mathrm{E}+00$ & $6.30 \mathrm{E}-06$ & $2.79 \mathrm{E}-07$ & $6.30 \mathrm{E}-06$ \\
\hline S-35 & 3.66E-06 & $0.00 \mathrm{E}+00$ & $0.00 \mathrm{E}+00$ & $3.50 \mathrm{E}-06$ & 1.62E-07 & $3.50 \mathrm{E}-06$ \\
\hline $\mathrm{Cl}-36$ & $1.88 \mathrm{E}-05$ & $7.85 \mathrm{E}-15$ & $5.26 \mathrm{E}-11$ & 1.09E-05 & $7.88 \mathrm{E}-06$ & 1.09E-05 \\
\hline $\mathrm{Cl}-38$ & 3.57E-06 & $3.80 \mathrm{E}-07$ & $2.98 \mathrm{E}-06$ & $2.15 \mathrm{E}-07$ & 2.11E-09 & $5.95 \mathrm{E}-07$ \\
\hline $\mathrm{Cl}-39$ & $2.75 \mathrm{E}-07$ & $0.00 \mathrm{E}+00$ & $0.00 \mathrm{E}+00$ & $2.71 \mathrm{E}-07$ & 3.93E-09 & $2.71 \mathrm{E}-07$ \\
\hline $\mathrm{Cl}-40$ & $4.18 \mathrm{E}-25$ & $0.00 \mathrm{E}+00$ & $0.00 \mathrm{E}+00$ & $4.18 \mathrm{E}-25$ & $0.00 \mathrm{E}+00$ & $4.18 \mathrm{E}-25$ \\
\hline Ar-37 & $3.04 \mathrm{E}-12$ & $3.04 \mathrm{E}-12$ & $0.00 \mathrm{E}+00$ & $0.00 \mathrm{E}+00$ & $0.00 \mathrm{E}+00$ & $3.04 \mathrm{E}-12$ \\
\hline Ar-39 & $0.00 \mathrm{E}+00$ & $0.00 \mathrm{E}+00$ & $0.00 \mathrm{E}+00$ & $0.00 \mathrm{E}+00$ & $0.00 \mathrm{E}+00$ & $0.00 \mathrm{E}+00$ \\
\hline Ar-41 & 1.83E-06 & 1.83E-06 & $0.00 \mathrm{E}+00$ & $0.00 \mathrm{E}+00$ & $0.00 \mathrm{E}+00$ & $1.83 \mathrm{E}-06$ \\
\hline $\mathrm{K}-40$ & 1.86E-04 & $3.41 \mathrm{E}-07$ & $2.28 \mathrm{E}-05$ & 1.56E-04 & 7.34E-06 & 1.56E-04 \\
\hline $\mathrm{K}-42$ & 1.96E-05 & $5.66 \mathrm{E}-07$ & $4.11 \mathrm{E}-06$ & 1.47E-05 & $1.52 \mathrm{E}-07$ & 1.53E-05 \\
\hline $\mathrm{K}-43$ & 4.19E-05 & $1.99 \mathrm{E}-06$ & $3.19 \mathrm{E}-05$ & 7.87E-06 & $1.27 \mathrm{E}-07$ & $9.86 \mathrm{E}-06$ \\
\hline Ca-41 & 1.47E-05 & $6.63 \mathrm{E}-12$ & $3.45 \mathrm{E}-09$ & 1.40E-05 & $6.62 \mathrm{E}-07$ & $1.40 \mathrm{E}-05$ \\
\hline Ca-45 & $8.14 \mathrm{E}-05$ & $3.45 \mathrm{E}-17$ & $3.53 \mathrm{E}-14$ & $7.78 \mathrm{E}-05$ & 3.63E-06 & $7.78 \mathrm{E}-05$ \\
\hline Ca-47 & 1.90E-04 & $2.29 \mathrm{E}-06$ & $1.05 \mathrm{E}-04$ & 7.99E-05 & 3.05E-06 & $8.22 \mathrm{E}-05$ \\
\hline Ca-49 & 1.73E-07 & $1.85 \mathrm{E}-08$ & $4.24 \mathrm{E}-09$ & $1.50 \mathrm{E}-07$ & 1.47E-10 & 1.69E-07 \\
\hline Sc-44 & $6.55 \mathrm{E}-05$ & $2.28 \mathrm{E}-06$ & $5.90 \mathrm{E}-05$ & $4.02 \mathrm{E}-06$ & 1.42E-07 & $6.30 \mathrm{E}-06$ \\
\hline Sc-44m & $9.23 \mathrm{E}-04$ & $5.64 \mathrm{E}-07$ & $8.53 \mathrm{E}-04$ & $5.49 \mathrm{E}-05$ & 1.52E-05 & $5.55 \mathrm{E}-05$ \\
\hline Sc-46 & $2.13 \mathrm{E}-03$ & $2.75 \mathrm{E}-06$ & $1.82 \mathrm{E}-03$ & $2.14 \mathrm{E}-04$ & $9.28 \mathrm{E}-05$ & $2.17 \mathrm{E}-04$ \\
\hline
\end{tabular}


Table 4. Ground release, Class F, $1 \mathrm{~m} / \mathrm{s}$ wind, $1 \mathrm{~km}$, no rain, continued.

\begin{tabular}{|c|c|c|c|c|c|c|}
\hline Nuclide & $\begin{array}{c}\text { 7-day } \\
\text { Total } \\
(1+2+3+4)\end{array}$ & $\begin{array}{c}\text { Cloud } \\
\text { shine } \\
(1)\end{array}$ & $\begin{array}{c}\text { Ground } \\
\text { shine } \\
(2)\end{array}$ & $\begin{array}{c}\text { Inhalation } \\
\text { (3) }\end{array}$ & $\begin{array}{c}\text { Resus- } \\
\text { pension } \\
(4)\end{array}$ & $\begin{array}{c}\text { Plume } \\
\text { Passage } \\
(1)+(3)\end{array}$ \\
\hline Sc-47 & 8.31E-05 & 1.46E-07 & $6.37 \mathrm{E}-05$ & $1.48 \mathrm{E}-05$ & 4.36E-06 & 1.49E-05 \\
\hline Sc-48 & 1.10E-03 & 4.47E-06 & $1.05 \mathrm{E}-03$ & $3.20 \mathrm{E}-05$ & 7.24E-06 & 3.65E-05 \\
\hline Sc-49 & 5.33E-07 & $5.96 \mathrm{E}-10$ & $3.98 \mathrm{E}-09$ & $5.23 \mathrm{E}-07$ & 4.77E-09 & 5.24E-07 \\
\hline Sc-50 & 4.82E-21 & $0.00 \mathrm{E}+00$ & $0.00 \mathrm{E}+00$ & $4.82 \mathrm{E}-21$ & $0.00 \mathrm{E}+00$ & 4.82E-21 \\
\hline Ti-44 & 3.97E-03 & $7.50 \mathrm{E}-07$ & $2.11 \mathrm{E}-03$ & 1.29E-03 & $5.70 \mathrm{E}-04$ & $1.29 \mathrm{E}-03$ \\
\hline Ti-45 & $2.18 \mathrm{E}-05$ & $8.54 \mathrm{E}-07$ & 1.93E-05 & 1.62E-06 & $4.55 \mathrm{E}-08$ & 2.47E-06 \\
\hline Ti-51 & $1.24 \mathrm{E}-10$ & $7.74 \mathrm{E}-11$ & $2.46 \mathrm{E}-11$ & $2.25 \mathrm{E}-11$ & $2.23 \mathrm{E}-15$ & $9.99 \mathrm{E}-11$ \\
\hline$V-48$ & $2.42 \mathrm{E}-03$ & 3.95E-06 & 2.31E-03 & 7.26E-05 & 2.92E-05 & 7.66E-05 \\
\hline$V-49$ & $3.60 \mathrm{E}-06$ & $1.23 \mathrm{E}-11$ & 7.27E-08 & $2.45 \mathrm{E}-06$ & $1.08 \mathrm{E}-06$ & $2.45 \mathrm{E}-06$ \\
\hline V-52 & $2.86 \mathrm{E}-12$ & $2.21 \mathrm{E}-12$ & $5.21 \mathrm{E}-13$ & $1.34 \mathrm{E}-13$ & $8.67 \mathrm{E}-18$ & $2.34 \mathrm{E}-12$ \\
\hline V-53 & $3.81 \mathrm{E}-22$ & $0.00 \mathrm{E}+00$ & $0.00 \mathrm{E}+00$ & 3.81E-22 & $0.00 \mathrm{E}+00$ & $3.81 \mathrm{E}-22$ \\
\hline $\mathrm{Cr}-49$ & 1.45E-06 & $6.26 \mathrm{E}-07$ & $4.66 \mathrm{E}-07$ & 3.56E-07 & $2.72 \mathrm{E}-10$ & $9.82 \mathrm{E}-07$ \\
\hline Cr-51 & 9.38E-06 & $6.67 \mathrm{E}-08$ & $5.22 \mathrm{E}-06$ & $3.92 \mathrm{E}-06$ & 1.75E-07 & 3.99E-06 \\
\hline Mn-52 & 4.43E-04 & $7.51 \mathrm{E}-06$ & $3.65 \mathrm{E}-04$ & $6.83 \mathrm{E}-05$ & 2.49E-06 & $7.58 \mathrm{E}-05$ \\
\hline Mn-52m & 7.23E-07 & $4.19 \mathrm{E}-07$ & $2.00 \mathrm{E}-07$ & 1.04E-07 & $1.51 \mathrm{E}-10$ & $5.23 \mathrm{E}-07$ \\
\hline Mn-53 & $6.14 \mathrm{E}-06$ & $3.68 \mathrm{E}-11$ & $2.65 \mathrm{E}-08$ & $5.84 \mathrm{E}-06$ & $2.75 \mathrm{E}-07$ & $5.84 \mathrm{E}-06$ \\
\hline Mn-54 & $2.24 \mathrm{E}-04$ & $1.84 \mathrm{E}-06$ & 1.39E-04 & 7.96E-05 & $3.73 \mathrm{E}-06$ & $8.14 \mathrm{E}-05$ \\
\hline Mn-56 & 1.19E-05 & $2.78 \mathrm{E}-06$ & 4.79E-06 & 4.36E-06 & $1.11 \mathrm{E}-08$ & 7.14E-06 \\
\hline Mn-57 & $3.24 \mathrm{E}-23$ & $1.89 \mathrm{E}-23$ & $4.50 \mathrm{E}-24$ & $8.92 \mathrm{E}-24$ & $0.00 \mathrm{E}+00$ & $2.78 \mathrm{E}-23$ \\
\hline Fe-52 & 4.21E-05 & $5.85 \mathrm{E}-06$ & $3.52 \mathrm{E}-05$ & $1.08 \mathrm{E}-06$ & 1.10E-08 & $6.93 \mathrm{E}-06$ \\
\hline Fe-55 & $1.57 \mathrm{E}-05$ & $4.86 \mathrm{E}-11$ & $3.69 \mathrm{E}-08$ & $1.50 \mathrm{E}-05$ & 7.04E-07 & $1.50 \mathrm{E}-05$ \\
\hline Fe-59 & 3.30E-04 & $2.59 \mathrm{E}-06$ & 1.73E-04 & 1.47E-04 & $6.71 \mathrm{E}-06$ & $1.50 \mathrm{E}-04$ \\
\hline Fe-60 & $3.45 \mathrm{E}-03$ & $9.32 \mathrm{E}-09$ & $1.26 \mathrm{E}-06$ & $3.29 \mathrm{E}-03$ & $1.55 \mathrm{E}-04$ & $3.29 \mathrm{E}-03$ \\
\hline Co-56 & 5.44E-04 & 8.36E-06 & $5.36 \mathrm{E}-04$ & $0.00 \mathrm{E}+00$ & $0.00 \mathrm{E}+00$ & 8.36E-06 \\
\hline Co-57 & 1.32E-04 & 2.67E-07 & $2.36 \mathrm{E}-05$ & 1.04E-04 & 4.85E-06 & 1.04E-04 \\
\hline Co-58 & 2.95E-04 & $2.13 \mathrm{E}-06$ & 1.59E-04 & $1.28 \mathrm{E}-04$ & $5.89 \mathrm{E}-06$ & $1.30 \mathrm{E}-04$ \\
\hline Co-58m & 2.07E-06 & 1.12E-09 & 7.99E-07 & $1.23 \mathrm{E}-06$ & $3.62 \mathrm{E}-08$ & $1.23 \mathrm{E}-06$ \\
\hline Co-60 & 3.01E-03 & $5.45 \mathrm{E}-06$ & $3.78 \mathrm{E}-04$ & $2.51 \mathrm{E}-03$ & $1.18 \mathrm{E}-04$ & $2.52 \mathrm{E}-03$ \\
\hline Co-60m & 1.16E-08 & $7.85 \mathrm{E}-11$ & 1.45E-09 & $9.60 \mathrm{E}-09$ & $4.44 \mathrm{E}-10$ & $9.68 \mathrm{E}-09$ \\
\hline Co-61 & $1.51 \mathrm{E}-06$ & $1.08 \mathrm{E}-07$ & $1.70 \mathrm{E}-07$ & $1.23 \mathrm{E}-06$ & 2.04E-09 & $1.34 \mathrm{E}-06$ \\
\hline Co-62m & 1.67E-08 & $0.00 \mathrm{E}+00$ & $0.00 \mathrm{E}+00$ & 1.67E-08 & $4.00 \mathrm{E}-12$ & 1.67E-08 \\
\hline $\mathrm{Ni}-56$ & 2.17E-04 & $3.74 \mathrm{E}-06$ & $2.13 \mathrm{E}-04$ & $0.00 \mathrm{E}+00$ & $0.00 \mathrm{E}+00$ & 3.74E-06 \\
\hline $\mathrm{Ni}-57$ & 1.14E-04 & $4.12 \mathrm{E}-06$ & 8.66E-05 & $2.30 \mathrm{E}-05$ & $5.14 \mathrm{E}-07$ & $2.71 \mathrm{E}-05$ \\
\hline $\mathrm{Ni}-59$ & 1.04E-05 & $8.15 \mathrm{E}-11$ & $6.96 \mathrm{E}-08$ & $9.90 \mathrm{E}-06$ & 4.67E-07 & $9.90 \mathrm{E}-06$ \\
\hline $\mathrm{Ni}-63$ & $2.85 \mathrm{E}-05$ & $0.00 \mathrm{E}+00$ & $0.00 \mathrm{E}+00$ & $2.72 \mathrm{E}-05$ & $1.28 \mathrm{E}-06$ & $2.72 \mathrm{E}-05$ \\
\hline $\mathrm{Ni}-65$ & 5.17E-06 & $8.44 \mathrm{E}-07$ & 1.43E-06 & $2.89 \mathrm{E}-06$ & 7.19E-09 & 3.73E-06 \\
\hline Cu-61 & 4.86E-06 & 1.35E-06 & $3.52 \mathrm{E}-06$ & $0.00 \mathrm{E}+00$ & $0.00 \mathrm{E}+00$ & 1.35E-06 \\
\hline Cu-62 & $1.51 \mathrm{E}-08$ & 9.09E-09 & $3.26 \mathrm{E}-09$ & 2.74E-09 & $4.61 \mathrm{E}-13$ & $1.18 \mathrm{E}-08$ \\
\hline Cu-64 & 7.97E-06 & $3.73 \mathrm{E}-07$ & $3.40 \mathrm{E}-06$ & $4.15 \mathrm{E}-06$ & 4.37E-08 & $4.52 \mathrm{E}-06$ \\
\hline Cu-66 & $7.40 \mathrm{E}-12$ & $0.00 \mathrm{E}+00$ & $0.00 \mathrm{E}+00$ & $7.40 \mathrm{E}-12$ & $6.52 \mathrm{E}-16$ & $7.40 \mathrm{E}-12$ \\
\hline Cu-67 & 1.00E-05 & $2.45 \mathrm{E}-07$ & $9.80 \mathrm{E}-06$ & $0.00 \mathrm{E}+00$ & $0.00 \mathrm{E}+00$ & $2.45 \mathrm{E}-07$ \\
\hline
\end{tabular}


Table 4. Ground release, Class F, $1 \mathrm{~m} / \mathrm{s}$ wind, $1 \mathrm{~km}$, no rain, continued.

\begin{tabular}{|c|c|c|c|c|c|c|}
\hline Nuclide & $\begin{array}{c}\text { 7-day } \\
\text { Total } \\
(1+2+3+4)\end{array}$ & $\begin{array}{c}\text { Cloud } \\
\text { shine } \\
(1)\end{array}$ & $\begin{array}{l}\text { Ground } \\
\text { shine } \\
(2)\end{array}$ & $\begin{array}{c}\text { Inhalation } \\
(3)\end{array}$ & $\begin{array}{l}\text { Resus- } \\
\text { pension } \\
\end{array}$ & $\begin{array}{c}\text { Plume } \\
\text { Passage } \\
(1)+(3)\end{array}$ \\
\hline $\mathrm{Zn}-62$ & $2.26 \mathrm{E}-05$ & $2.82 \mathrm{E}-06$ & $1.92 \mathrm{E}-05$ & 5.92E-07 & 4.83E-09 & $3.41 \mathrm{E}-06$ \\
\hline Zn-63 & 4.36E-07 & $0.00 \mathrm{E}+00$ & $0.00 \mathrm{E}+00$ & 4.36E-07 & $2.84 \mathrm{E}-10$ & 4.36E-07 \\
\hline Zn-65 & $3.40 \mathrm{E}-04$ & $1.27 \mathrm{E}-06$ & $9.03 \mathrm{E}-05$ & 2.37E-04 & 1.11E-05 & $2.38 \mathrm{E}-04$ \\
\hline Zn-69 & $3.20 \mathrm{E}-07$ & 4.97E-12 & $4.64 \mathrm{E}-12$ & $3.20 \mathrm{E}-07$ & $3.10 \mathrm{E}-10$ & $3.20 \mathrm{E}-07$ \\
\hline $\mathrm{Zn}-69 \mathrm{~m}$ & $2.09 \mathrm{E}-05$ & $8.20 \mathrm{E}-07$ & $8.29 \mathrm{E}-06$ & 1.16E-05 & 1.34E-07 & 1.24E-05 \\
\hline $\mathrm{Zn}-71 \mathrm{~m}$ & 5.55E-06 & $0.00 \mathrm{E}+00$ & $0.00 \mathrm{E}+00$ & $5.53 \mathrm{E}-06$ & 2.09E-08 & $5.53 \mathrm{E}-06$ \\
\hline Ga-66 & $3.39 \mathrm{E}-05$ & $5.38 \mathrm{E}-06$ & $2.85 \mathrm{E}-05$ & $0.00 \mathrm{E}+00$ & $0.00 \mathrm{E}+00$ & $5.38 \mathrm{E}-06$ \\
\hline Ga-67 & 1.44E-05 & $3.06 \mathrm{E}-07$ & $1.41 \mathrm{E}-05$ & $0.00 \mathrm{E}+00$ & $0.00 \mathrm{E}+00$ & 3.06E-07 \\
\hline Ga-68 & $2.83 \mathrm{E}-06$ & $9.20 \mathrm{E}-07$ & $9.51 \mathrm{E}-07$ & $9.59 \mathrm{E}-07$ & 1.10E-09 & $1.88 \mathrm{E}-06$ \\
\hline Ga-70 & 4.74E-08 & $0.00 \mathrm{E}+00$ & $0.00 \mathrm{E}+00$ & $4.74 \mathrm{E}-08$ & $1.72 \mathrm{E}-11$ & $4.74 \mathrm{E}-08$ \\
\hline Ga-72 & 8.01E-05 & $5.95 \mathrm{E}-06$ & $4.95 \mathrm{E}-05$ & $2.44 \mathrm{E}-05$ & $2.79 \mathrm{E}-07$ & 3.04E-05 \\
\hline Ge-68 & 1.63E-04 & 1.09E-06 & 1.61E-04 & $1.14 \mathrm{E}-06$ & $9.71 \mathrm{E}-08$ & 2.23E-06 \\
\hline Ge-69 & 8.65E-06 & $0.00 \mathrm{E}+00$ & $0.00 \mathrm{E}+00$ & 8.46E-06 & 1.92E-07 & $8.46 \mathrm{E}-06$ \\
\hline Ge-71 & 1.61E-07 & $1.92 \mathrm{E}-10$ & $1.60 \mathrm{E}-07$ & $0.00 \mathrm{E}+00$ & $0.00 \mathrm{E}+00$ & $1.92 \mathrm{E}-10$ \\
\hline Ge-75 & 7.48E-07 & $0.00 \mathrm{E}+00$ & $0.00 \mathrm{E}+00$ & 7.47E-07 & $1.04 \mathrm{E}-09$ & 7.47E-07 \\
\hline Ge-77 & $3.58 \mathrm{E}-05$ & $2.13 \mathrm{E}-06$ & $1.69 \mathrm{E}-05$ & 1.65E-05 & 2.31E-07 & $1.86 \mathrm{E}-05$ \\
\hline As-72 & $6.90 \mathrm{E}-05$ & $3.74 \mathrm{E}-06$ & $6.53 \mathrm{E}-05$ & $0.00 \mathrm{E}+00$ & $0.00 \mathrm{E}+00$ & $3.74 \mathrm{E}-06$ \\
\hline As-73 & 1.57E-06 & $9.50 \mathrm{E}-09$ & $1.56 \mathrm{E}-06$ & $0.00 \mathrm{E}+00$ & $0.00 \mathrm{E}+00$ & $9.50 \mathrm{E}-09$ \\
\hline As-74 & $2.13 \mathrm{E}-04$ & 1.63E-06 & $1.15 \mathrm{E}-04$ & $9.24 \mathrm{E}-05$ & 3.99E-06 & $9.40 \mathrm{E}-05$ \\
\hline As-76 & 6.57E-05 & 8.99E-07 & $1.56 \mathrm{E}-05$ & 4.83E-05 & 8.66E-07 & 4.92E-05 \\
\hline As-77 & 1.43E-05 & $1.79 \mathrm{E}-08$ & $4.85 \mathrm{E}-07$ & 1.35E-05 & 3.06E-07 & 1.35E-05 \\
\hline As-78 & 2.87E-06 & $0.00 \mathrm{E}+00$ & $0.00 \mathrm{E}+00$ & 2.87E-06 & 4.37E-09 & 2.87E-06 \\
\hline Se-73 & 1.30E-05 & $2.03 \mathrm{E}-06$ & 1.10E-05 & $0.00 \mathrm{E}+00$ & $0.00 \mathrm{E}+00$ & 2.03E-06 \\
\hline Se-75 & $75 \mathrm{E}-04$ & 8.32E-07 & 7.05E-05 & 9.91E-05 & 4.61E-06 & 9.99E-05 \\
\hline Se-79 & 1.17E-04 & $0.00 \mathrm{E}+00$ & $0.00 \mathrm{E}+00$ & 1.12E-04 & $5.26 \mathrm{E}-06$ & 1.12E-04 \\
\hline $\mathrm{Br}-77$ & $57 E-04$ & $2.90 \mathrm{E}-07$ & $1.56 \mathrm{E}-04$ & $0.00 \mathrm{E}+00$ & $0.00 \mathrm{E}+00$ & $2.90 \mathrm{E}-07$ \\
\hline $\mathrm{Br}-80$ & $3.48 \mathrm{E}-08$ & $3.30 \mathrm{E}-09$ & $2.23 \mathrm{E}-08$ & $9.15 \mathrm{E}-09$ & $4.21 \mathrm{E}-11$ & $1.25 \mathrm{E}-08$ \\
\hline $\mathrm{Br}-80 \mathrm{~m}$ & 5.33E-06 & $6.32 \mathrm{E}-08$ & $3.70 \mathrm{E}-06$ & $1.47 \mathrm{E}-06$ & $9.58 \mathrm{E}-08$ & $1.53 \mathrm{E}-06$ \\
\hline $\mathrm{Br}-82$ & $8.53 \mathrm{E}-04$ & $2.46 \mathrm{E}-06$ & $8.42 \mathrm{E}-04$ & $6.41 \mathrm{E}-06$ & $2.12 \mathrm{E}-06$ & 8.87E-06 \\
\hline $\mathrm{Br}-83$ & 1.64E-07 & $4.83 \mathrm{E}-09$ & $1.59 \mathrm{E}-07$ & $0.00 \mathrm{E}+00$ & $0.00 \mathrm{E}+00$ & $4.83 \mathrm{E}-09$ \\
\hline $\mathrm{Br}-84$ & $2.89 \mathrm{E}-06$ & $3.55 \mathrm{E}-07$ & $2.53 \mathrm{E}-06$ & $0.00 \mathrm{E}+00$ & $0.00 \mathrm{E}+00$ & $3.55 \mathrm{E}-07$ \\
\hline $\mathrm{Kr}-79$ & $5.58 \mathrm{E}-07$ & $5.58 \mathrm{E}-07$ & $0.00 \mathrm{E}+00$ & $0.00 \mathrm{E}+00$ & $0.00 \mathrm{E}+00$ & 5.58E-07 \\
\hline $\mathrm{Kr}-81$ & $2.30 \mathrm{E}-08$ & $2.30 \mathrm{E}-08$ & $0.00 \mathrm{E}+00$ & $0.00 \mathrm{E}+00$ & $0.00 \mathrm{E}+00$ & $2.30 \mathrm{E}-08$ \\
\hline $\mathrm{Kr}-83 \mathrm{~m}$ & $1.30 \mathrm{E}-10$ & $1.30 \mathrm{E}-10$ & $0.00 \mathrm{E}+00$ & $0.00 \mathrm{E}+00$ & $0.00 \mathrm{E}+00$ & $1.30 \mathrm{E}-10$ \\
\hline $\mathrm{Kr}-85$ & 5.05E-09 & $5.05 \mathrm{E}-09$ & $0.00 \mathrm{E}+00$ & $0.00 \mathrm{E}+00$ & $0.00 \mathrm{E}+00$ & $5.05 \mathrm{E}-09$ \\
\hline $\mathrm{Kr}-85 \mathrm{~m}$ & $3.00 \mathrm{E}-07$ & $3.00 \mathrm{E}-07$ & $0.00 \mathrm{E}+00$ & $0.00 \mathrm{E}+00$ & $0.00 \mathrm{E}+00$ & $3.00 \mathrm{E}-07$ \\
\hline $\mathrm{Kr}-87$ & $9.99 \mathrm{E}-07$ & $9.99 \mathrm{E}-07$ & $0.00 \mathrm{E}+00$ & $4.40 \mathrm{E}-20$ & $2.07 \mathrm{E}-21$ & 9.99E-07 \\
\hline $\mathrm{Kr}-88$ & $6.38 \mathrm{E}-06$ & $4.88 \mathrm{E}-06$ & $4.08 \mathrm{E}-07$ & 1.09E-06 & $3.33 \mathrm{E}-10$ & 5.97E-06 \\
\hline $\mathrm{Rb}-81$ & $4.80 \mathrm{E}-06$ & 1.06E-06 & $3.74 \mathrm{E}-06$ & $0.00 \mathrm{E}+00$ & $0.00 \mathrm{E}+00$ & 1.06E-06 \\
\hline $\mathrm{Rb}-82$ & $5.08 \mathrm{E}-24$ & $4.10 \mathrm{E}-24$ & $9.88 \mathrm{E}-25$ & $0.00 \mathrm{E}+00$ & $0.00 \mathrm{E}+00$ & $4.10 \mathrm{E}-24$ \\
\hline $\mathrm{Rb}-83$ & 8.52E-05 & 1.06E-06 & 8.41E-05 & $0.00 E+00$ & $0.00 \mathrm{E}+00$ & 1.06E-06 \\
\hline
\end{tabular}


Table 4. Ground release, Class F, $1 \mathrm{~m} / \mathrm{s}$ wind, $1 \mathrm{~km}$, no rain, continued.

\begin{tabular}{|c|c|c|c|c|c|c|}
\hline Nuclide & $\begin{array}{c}\text { 7-day } \\
\text { Total } \\
(1+2+3+4)\end{array}$ & $\begin{array}{c}\text { Cloud } \\
\text { shine } \\
(1)\end{array}$ & $\begin{array}{l}\text { Ground } \\
\text { shine } \\
(2)\end{array}$ & $\begin{array}{c}\text { Inhalation } \\
\text { (3) }\end{array}$ & $\begin{array}{c}\text { Resus- } \\
\text { pension } \\
(4)\end{array}$ & $\begin{array}{c}\text { Plume } \\
\text { Passage } \\
(1)+(3)\end{array}$ \\
\hline $\mathrm{Rb}-84$ & 2.02E-04 & 1.95E-06 & $1.40 \mathrm{E}-04$ & 5.72E-05 & 2.57E-06 & 5.92E-05 \\
\hline $\mathrm{Rb}-86$ & $9.78 \mathrm{E}-05$ & 2.07E-07 & $1.32 \mathrm{E}-05$ & 8.09E-05 & $3.51 \mathrm{E}-06$ & 8.11E-05 \\
\hline $\mathrm{Rb}-87$ & 2.97E-05 & $0.00 \mathrm{E}+00$ & $0.00 \mathrm{E}+00$ & $2.84 \mathrm{E}-05$ & 1.34E-06 & $2.84 \mathrm{E}-05$ \\
\hline $\mathrm{Rb}-88$ & $1.75 \mathrm{E}-07$ & 7.62E-08 & $2.69 \mathrm{E}-08$ & 7.17E-08 & $2.20 \mathrm{E}-11$ & $1.48 \mathrm{E}-07$ \\
\hline $\mathrm{Rb}-89$ & 2.07E-07 & $1.45 \mathrm{E}-07$ & 4.96E-08 & $1.20 \mathrm{E}-08$ & $5.63 \mathrm{E}-10$ & 1.57E-07 \\
\hline Sr-82 & 1.73E-04 & 2.32E-06 & $1.71 \mathrm{E}-04$ & $0.00 \mathrm{E}+00$ & $0.00 \mathrm{E}+00$ & 2.32E-06 \\
\hline Sr-85 & 1.10E-04 & $1.08 \mathrm{E}-06$ & $8.53 \mathrm{E}-05$ & $2.23 \mathrm{E}-05$ & 1.03E-06 & $2.34 \mathrm{E}-05$ \\
\hline Sr-85m & $5.20 \mathrm{E}-07$ & $2.15 \mathrm{E}-07$ & $2.97 \mathrm{E}-07$ & 7.80E-09 & $6.64 \mathrm{E}-10$ & 2.23E-07 \\
\hline Sr-87m & $1.61 \mathrm{E}-06$ & $4.90 \mathrm{E}-07$ & $1.12 \mathrm{E}-06$ & 1.57E-22 & $2.61 \mathrm{E}-23$ & 4.90E-07 \\
\hline Sr-89 & 6.17E-05 & $3.01 \mathrm{E}-10$ & $2.15 \mathrm{E}-08$ & $5.90 \mathrm{E}-05$ & 2.69E-06 & 5.90E-05 \\
\hline Sr-90 & 4.69E-03 & $0.00 \mathrm{E}+00$ & $0.00 \mathrm{E}+00$ & 4.47E-03 & $2.13 \mathrm{E}-04$ & 4.47E-03 \\
\hline Sr-91 & $2.45 \mathrm{E}-05$ & $1.78 \mathrm{E}-06$ & $1.30 \mathrm{E}-05$ & $9.54 \mathrm{E}-06$ & $2.25 \mathrm{E}-07$ & 1.13E-05 \\
\hline Sr-92 & $9.62 \mathrm{E}-06$ & $2.20 \mathrm{E}-06$ & $4.72 \mathrm{E}-06$ & 2.67E-06 & $3.47 \mathrm{E}-08$ & 4.87E-06 \\
\hline$Y-86$ & 7.76E-05 & 7.56E-06 & $7.00 \mathrm{E}-05$ & $0.00 \mathrm{E}+00$ & $0.00 \mathrm{E}+00$ & 7.56E-06 \\
\hline Y-87 & 7.27E-05 & $1.13 \mathrm{E}-06$ & $7.16 \mathrm{E}-05$ & $2.59 \mathrm{E}-23$ & $2.49 \mathrm{E}-22$ & 1.13E-06 \\
\hline Y-88 & 7.52E-04 & $6.25 \mathrm{E}-06$ & $4.06 \mathrm{E}-04$ & $3.25 \mathrm{E}-04$ & $1.51 \mathrm{E}-05$ & $3.31 \mathrm{E}-04$ \\
\hline$Y-90$ & 1.06E-04 & $0.00 \mathrm{E}+00$ & $0.00 \mathrm{E}+00$ & 1.03E-04 & $2.98 \mathrm{E}-06$ & 1.03E-04 \\
\hline Y-90m & $9.55 \mathrm{E}-06$ & $1.02 \mathrm{E}-06$ & $2.60 \mathrm{E}-06$ & $5.78 \mathrm{E}-06$ & 1.56E-07 & 6.80E-06 \\
\hline Y-91 & 5.95E-04 & 7.85E-09 & $5.30 \mathrm{E}-07$ & $5.68 \mathrm{E}-04$ & $2.61 \mathrm{E}-05$ & $5.68 \mathrm{E}-04$ \\
\hline Y-91m & 1.11E-06 & 3.87E-07 & $3.17 \mathrm{E}-07$ & 3.91E-07 & $1.54 \mathrm{E}-08$ & 7.78E-07 \\
\hline Y-92 & $1.26 \mathrm{E}-05$ & $4.31 \mathrm{E}-07$ & $1.05 \mathrm{E}-06$ & $1.11 \mathrm{E}-05$ & $3.80 \mathrm{E}-08$ & $1.15 \mathrm{E}-05$ \\
\hline$Y-93$ & 3.17E-05 & $1.86 \mathrm{E}-07$ & 1.17E-06 & 3.01E-05 & 2.63E-07 & 3.03E-05 \\
\hline Y-94 & $1.01 \mathrm{E}-07$ & $0.00 \mathrm{E}+00$ & $0.00 \mathrm{E}+00$ & $1.01 \mathrm{E}-07$ & $3.31 \mathrm{E}-11$ & $1.01 \mathrm{E}-07$ \\
\hline Zr-86 & 8.90E-05 & $1.01 \mathrm{E}-06$ & $8.80 \mathrm{E}-05$ & $0.00 \mathrm{E}+00$ & $0.00 \mathrm{E}+00$ & 1.01E-06 \\
\hline $\mathrm{Zr}-88$ & 7.69E-05 & 8.09E-07 & $7.57 \mathrm{E}-05$ & $1.12 \mathrm{E}-07$ & $2.23 \mathrm{E}-07$ & $9.21 \mathrm{E}-07$ \\
\hline $\mathrm{Zr}-89$ & $1.29 \mathrm{E}-04$ & $2.50 \mathrm{E}-06$ & 9.97E-05 & $2.65 \mathrm{E}-05$ & $8.24 \mathrm{E}-07$ & $2.90 \mathrm{E}-05$ \\
\hline Zr-93 & 1.01E-03 & $2.12 \mathrm{E}-15$ & $8.49 \mathrm{E}-11$ & $9.68 \mathrm{E}-04$ & 4.56E-05 & $9.68 \mathrm{E}-04$ \\
\hline Zr-95 & $3.23 \mathrm{E}-04$ & $1.61 \mathrm{E}-06$ & $1.28 \mathrm{E}-04$ & $1.85 \mathrm{E}-04$ & 8.63E-06 & 1.87E-04 \\
\hline $\mathrm{Zr}-97$ & $9.21 \mathrm{E}-05$ & $2.52 \mathrm{E}-06$ & $3.70 \mathrm{E}-05$ & $5.19 \mathrm{E}-05$ & $6.93 \mathrm{E}-07$ & $5.44 \mathrm{E}-05$ \\
\hline $\mathrm{Nb}-90$ & 1.16E-04 & $9.26 \mathrm{E}-06$ & 7.77E-05 & $2.90 \mathrm{E}-05$ & 3.41E-07 & 3.83E-05 \\
\hline $\mathrm{Nb}-92 \mathrm{~m}$ & $1.55 \mathrm{E}-04$ & $2.12 \mathrm{E}-06$ & $1.26 \mathrm{E}-04$ & $2.55 \mathrm{E}-05$ & 1.04E-06 & $2.76 \mathrm{E}-05$ \\
\hline $\mathrm{Nb}-93 \mathrm{~m}$ & $9.03 \mathrm{E}-12$ & $2.11 \mathrm{E}-12$ & $1.23 \mathrm{E}-12$ & $5.58 \mathrm{E}-12$ & $1.22 \mathrm{E}-13$ & 7.69E-12 \\
\hline $\mathrm{Nb}-94 \mathrm{~m}$ & $3.67 \mathrm{E}-04$ & $2.86 \mathrm{E}-10$ & $1.72 \mathrm{E}-07$ & $3.50 \mathrm{E}-04$ & $1.65 \mathrm{E}-05$ & $3.50 \mathrm{E}-04$ \\
\hline $\mathrm{Nb}-94$ & 4.90E-03 & $3.45 \mathrm{E}-06$ & $2.65 \mathrm{E}-04$ & $4.43 \mathrm{E}-03$ & 2.09E-04 & 4.43E-03 \\
\hline $\mathrm{Nb}-95$ & 1.93E-04 & 1.67E-06 & $1.21 \mathrm{E}-04$ & $6.75 \mathrm{E}-05$ & $3.05 \mathrm{E}-06$ & $6.92 \mathrm{E}-05$ \\
\hline $\mathrm{Nb}-95 \mathrm{~m}$ & 4.39E-05 & $1.28 \mathrm{E}-07$ & $1.20 \mathrm{E}-05$ & 3.06E-05 & 1.09E-06 & 3.07E-05 \\
\hline $\mathrm{Nb}-96$ & $1.14 \mathrm{E}-04$ & 5.17E-06 & 7.97E-05 & 2.87E-05 & 4.76E-07 & $3.39 \mathrm{E}-05$ \\
\hline $\mathrm{Nb}-97$ & $2.24 \mathrm{E}-06$ & $6.87 \mathrm{E}-07$ & 7.23E-07 & 8.24E-07 & 1.00E-09 & $1.51 \mathrm{E}-06$ \\
\hline $\mathrm{Nb}-97 \mathrm{~m}$ & $3.14 \mathrm{E}-08$ & 9.67E-09 & 1.02E-08 & 1.16E-08 & $1.41 \mathrm{E}-11$ & 2.13E-08 \\
\hline $\mathrm{Nb}-98$ & $8.43 \mathrm{E}-07$ & $0.00 \mathrm{E}+00$ & $0.00 \mathrm{E}+00$ & $8.42 \mathrm{E}-07$ & $7.38 \mathrm{E}-10$ & 8.42E-07 \\
\hline Mo-93 & 4.26E-04 & 1.60E-09 & $9.68 \mathrm{E}-07$ & 4.06E-04 & $1.91 \mathrm{E}-05$ & 4.06E-04 \\
\hline
\end{tabular}


Table 4. Ground release, Class F, $1 \mathrm{~m} / \mathrm{s}$ wind, $1 \mathrm{~km}$, no rain, continued.

\begin{tabular}{|c|c|c|c|c|c|c|}
\hline Nuclide & $\begin{array}{c}\text { 7-day } \\
\text { Total } \\
(1+2+3+4)\end{array}$ & $\begin{array}{c}\text { Cloud } \\
\text { shine } \\
(1)\end{array}$ & $\begin{array}{l}\text { Ground } \\
\text { shine } \\
(2)\end{array}$ & $\begin{array}{c}\text { Inhalation } \\
(3)\end{array}$ & $\begin{array}{l}\text { Resus- } \\
\text { pension } \\
\end{array}$ & $\begin{array}{c}\text { Plume } \\
\text { Passage } \\
(1)+(3)\end{array}$ \\
\hline Mo-93m & 5.53E-06 & $4.32 \mathrm{E}-17$ & $2.04 \mathrm{E}-13$ & $5.50 \mathrm{E}-06$ & 3.44E-08 & $5.50 \mathrm{E}-06$ \\
\hline Mo-99 & 7.23E-05 & $3.65 \mathrm{E}-07$ & $2.23 \mathrm{E}-05$ & 4.82E-05 & $1.41 \mathrm{E}-06$ & $4.86 \mathrm{E}-05$ \\
\hline Mo-101 & 2.69E-07 & $1.56 \mathrm{E}-07$ & $6.42 \mathrm{E}-08$ & 4.84E-08 & $1.41 \mathrm{E}-11$ & 2.04E-07 \\
\hline Tc-95 & 2.72E-05 & 1.65E-06 & $2.23 \mathrm{E}-05$ & $3.24 \mathrm{E}-06$ & $4.82 \mathrm{E}-08$ & $4.89 \mathrm{E}-06$ \\
\hline Tc-95m & 1.17E-04 & 1.44E-06 & $1.15 \mathrm{E}-04$ & 5.87E-09 & 4.35E-09 & $1.45 \mathrm{E}-06$ \\
\hline Tc-96 & 2.85E-04 & $5.45 \mathrm{E}-06$ & $2.49 \mathrm{E}-04$ & $2.98 \mathrm{E}-05$ & $1.01 \mathrm{E}-06$ & 3.53E-05 \\
\hline Tc-96m & $2.30 \mathrm{E}-06$ & $6.13 \mathrm{E}-08$ & 2.07E-06 & $1.58 \mathrm{E}-07$ & 8.30E-09 & $2.19 \mathrm{E}-07$ \\
\hline Tc-97 & 1.29E-05 & 1.99E-09 & 1.07E-06 & 1.13E-05 & 5.31E-07 & 1.13E-05 \\
\hline Tc-97m & $9.06 \mathrm{E}-07$ & 2.44E-09 & 9.04E-07 & $4.38 \mathrm{E}-16$ & $8.81 \mathrm{E}-16$ & $2.44 \mathrm{E}-09$ \\
\hline Tc-98 & 4.83E-04 & $3.03 E-06$ & $2.38 \mathrm{E}-04$ & $2.31 \mathrm{E}-04$ & 1.09E-05 & $2.34 \mathrm{E}-04$ \\
\hline Tc-99 & 8.34E-05 & $1.11 \mathrm{E}-12$ & $1.04 \mathrm{E}-10$ & 7.96E-05 & $3.75 \mathrm{E}-06$ & 7.96E-05 \\
\hline Tc-99m & 1.73E-06 & $2.40 \mathrm{E}-07$ & $1.16 \mathrm{E}-06$ & 3.32E-07 & 1.85E-09 & 5.72E-07 \\
\hline Tc-101 & $3.20 \mathrm{E}-08$ & $1.72 \mathrm{E}-08$ & 7.31E-09 & 7.45E-09 & $1.82 \mathrm{E}-12$ & $2.47 \mathrm{E}-08$ \\
\hline Ru-97 & $2.68 \mathrm{E}-05$ & 4.84E-07 & $2.08 \mathrm{E}-05$ & $5.30 \mathrm{E}-06$ & $1.58 \mathrm{E}-07$ & $5.78 \mathrm{E}-06$ \\
\hline Ru-103 & 1.89E-04 & $1.02 \mathrm{E}-06$ & 7.87E-05 & 1.04E-04 & 4.72E-06 & 1.05E-04 \\
\hline Ru-105 & $1.25 \mathrm{E}-05$ & 1.39E-06 & $5.05 \mathrm{E}-06$ & $6.05 \mathrm{E}-06$ & $5.52 \mathrm{E}-08$ & 7.44E-06 \\
\hline Ru-106 & $5.83 \mathrm{E}-03$ & 4.44E-07 & $3.49 \mathrm{E}-05$ & $5.54 \mathrm{E}-03$ & $2.60 \mathrm{E}-04$ & $5.54 \mathrm{E}-03$ \\
\hline Rh-101 & 4.55E-04 & $0.00 \mathrm{E}+00$ & $0.00 \mathrm{E}+00$ & $4.35 \mathrm{E}-04$ & $2.05 \mathrm{E}-05$ & $4.35 \mathrm{E}-04$ \\
\hline $\mathrm{Rh}-101 \mathrm{~m}$ & 9.02E-06 & $0.00 \mathrm{E}+00$ & $0.00 \mathrm{E}+00$ & $8.72 \mathrm{E}-06$ & 2.99E-07 & 8.72E-06 \\
\hline Rh-102 & $1.43 \mathrm{E}-03$ & $0.00 \mathrm{E}+00$ & $0.00 \mathrm{E}+00$ & 1.37E-03 & $6.42 \mathrm{E}-05$ & 1.37E-03 \\
\hline $\mathrm{Rh}-102 \mathrm{~m}$ & $2.67 \mathrm{E}-04$ & $0.00 \mathrm{E}+00$ & $0.00 \mathrm{E}+00$ & $2.55 \mathrm{E}-04$ & 1.19E-05 & $2.55 \mathrm{E}-04$ \\
\hline $\mathrm{Rh}-103 \mathrm{~m}$ & 3.89E-08 & $1.64 \mathrm{E}-10$ & $8.25 \mathrm{E}-10$ & $3.78 \mathrm{E}-08$ & $3.61 \mathrm{E}-11$ & $3.80 \mathrm{E}-08$ \\
\hline Rh-105 & 1.64E-05 & $1.60 \mathrm{E}-07$ & 4.04E-06 & 1.19E-05 & $2.56 \mathrm{E}-07$ & $1.21 \mathrm{E}-05$ \\
\hline Rh-106 & $0.00 \mathrm{E}+00$ & $0.00 \mathrm{E}+00$ & $0.00 \mathrm{E}+00$ & $0.00 \mathrm{E}+00$ & $0.00 \mathrm{E}+00$ & $0.00 \mathrm{E}+00$ \\
\hline $\mathrm{Pd}-103$ & 1.72E-06 & 4.01E-09 & 1.65E-06 & 5.96E-08 & 4.17E-09 & $6.36 \mathrm{E}-08$ \\
\hline Pd-107 & 56E-04 & $0.00 \mathrm{E}+00$ & $E+00$ & $1.49 \mathrm{E}-04$ & 7.02E-06 & 1.49E-04 \\
\hline Pd-109 & $1.51 \mathrm{E}-05$ & $1.36 \mathrm{E}-09$ & $1.32 \mathrm{E}-08$ & $1.49 \mathrm{E}-05$ & 1.64E-07 & 1.49E-05 \\
\hline $\mathrm{Ag}-106$ & $6.11 \mathrm{E}-08$ & $0.00 \mathrm{E}+00$ & $0.00 \mathrm{E}+00$ & $6.11 \mathrm{E}-08$ & $2.51 \mathrm{E}-11$ & $6.11 \mathrm{E}-08$ \\
\hline $\mathrm{Ag}-106 \mathrm{~m}$ & $4.22 \mathrm{E}-04$ & $6.06 \mathrm{E}-06$ & $3.48 \mathrm{E}-04$ & $6.59 \mathrm{E}-05$ & $2.61 \mathrm{E}-06$ & $7.20 \mathrm{E}-05$ \\
\hline $\mathrm{Ag}-108$ & $.06 \mathrm{E}-17$ & $6.84 \mathrm{E}-18$ & $1.65 \mathrm{E}-18$ & $1.21 \mathrm{E}-17$ & $0.00 \mathrm{E}+00$ & $1.89 \mathrm{E}-17$ \\
\hline $\mathrm{Ag}-108 \mathrm{~m}$ & $3.56 \mathrm{E}-03$ & $3.46 \mathrm{E}-06$ & $2.78 \mathrm{E}-04$ & $3.13 \mathrm{E}-03$ & $1.48 \mathrm{E}-04$ & $3.13 \mathrm{E}-03$ \\
\hline $\mathrm{Ag}-109 \mathrm{~m}$ & $0.00 \mathrm{E}+00$ & $0.00 \mathrm{E}+00$ & $0.00 \mathrm{E}+00$ & $0.00 \mathrm{E}+00$ & $0.00 \mathrm{E}+00$ & $0.00 \mathrm{E}+00$ \\
\hline $\mathrm{Ag}-110$ & $0.00 \mathrm{E}+00$ & $0.00 \mathrm{E}+00$ & $0.00 \mathrm{E}+00$ & $0.00 \mathrm{E}+00$ & $0.00 \mathrm{E}+00$ & $0.00 \mathrm{E}+00$ \\
\hline $\mathrm{Ag}-110 \mathrm{~m}$ & $1.44 \mathrm{E}-03$ & $6.00 \mathrm{E}-06$ & 4.43E-04 & $9.46 \mathrm{E}-04$ & $4.43 \mathrm{E}-05$ & $9.52 \mathrm{E}-04$ \\
\hline $\mathrm{Ag}-111$ & $8.13 \mathrm{E}-05$ & $5.55 \mathrm{E}-08$ & $3.47 \mathrm{E}-06$ & 7.49E-05 & $2.90 \mathrm{E}-06$ & $7.50 \mathrm{E}-05$ \\
\hline Cd-109 & $4.10 \mathrm{E}-04$ & 4.65E-09 & $1.57 \mathrm{E}-06$ & $3.90 \mathrm{E}-04$ & 1.83E-05 & $3.90 \mathrm{E}-04$ \\
\hline Cd-113 & $0.00 \mathrm{E}+00$ & $0.00 \mathrm{E}+00$ & $0.00 \mathrm{E}+00$ & $0.00 \mathrm{E}+00$ & $0.00 \mathrm{E}+00$ & $0.00 \mathrm{E}+00$ \\
\hline $\mathrm{Cd}-113 \mathrm{~m}$ & $4.83 \mathrm{E}-03$ & $0.00 \mathrm{E}+00$ & $0.00 \mathrm{E}+00$ & $4.62 \mathrm{E}-03$ & $2.17 \mathrm{E}-04$ & $4.62 \mathrm{E}-03$ \\
\hline $\mathrm{Cd}-115$ & $8.27 \mathrm{E}-05$ & $4.84 \mathrm{E}-07$ & $2.59 \mathrm{E}-05$ & $5.49 \mathrm{E}-05$ & $1.51 \mathrm{E}-06$ & $5.54 \mathrm{E}-05$ \\
\hline $\mathrm{Cd}-115 \mathrm{~m}$ & $3.36 \mathrm{E}-06$ & $4.81 \mathrm{E}-08$ & $3.31 \mathrm{E}-06$ & $2.35 \mathrm{E}-22$ & $4.68 \mathrm{E}-22$ & $4.81 \mathrm{E}-08$ \\
\hline Cd-117 & $6.45 \mathrm{E}-06$ & 1.89E-06 & 4.56E-06 & $0.00 \mathrm{E}+00$ & $0.00 \mathrm{E}+00$ & 1.89E-06 \\
\hline
\end{tabular}


Table 4. Ground release, Class F, $1 \mathrm{~m} / \mathrm{s}$ wind, $1 \mathrm{~km}$, no rain, continued.

\begin{tabular}{|c|c|c|c|c|c|c|}
\hline Nuclide & $\begin{array}{c}\text { 7-day } \\
\text { Total } \\
(1+2+3+4)\end{array}$ & $\begin{array}{c}\text { Cloud } \\
\text { shine } \\
(1)\end{array}$ & $\begin{array}{l}\text { Ground } \\
\text { shine } \\
(2)\end{array}$ & $\begin{array}{c}\text { Inhalation } \\
(3)\end{array}$ & $\begin{array}{c}\text { Resus- } \\
\text { pension } \\
(4)\end{array}$ & $\begin{array}{c}\text { Plume } \\
\text { Passage } \\
(1)+(3)\end{array}$ \\
\hline $\mathrm{Cd}-117 \mathrm{~m}$ & 1.56E-05 & $4.51 \mathrm{E}-06$ & 1.11E-05 & $0.00 \mathrm{E}+00$ & $0.00 \mathrm{E}+00$ & $4.51 \mathrm{E}-06$ \\
\hline $\ln -111$ & $3.58 \mathrm{E}-05$ & $8.26 \mathrm{E}-07$ & $3.50 \mathrm{E}-05$ & $0.00 \mathrm{E}+00$ & $0.00 \mathrm{E}+00$ & $8.26 \mathrm{E}-07$ \\
\hline In-113m & 7.75E-07 & $3.14 \mathrm{E}-07$ & 4.61E-07 & $0.00 \mathrm{E}+00$ & $0.00 \mathrm{E}+00$ & $3.14 \mathrm{E}-07$ \\
\hline $\ln -114$ & $0.00 \mathrm{E}+00$ & $0.00 \mathrm{E}+00$ & $0.00 \mathrm{E}+00$ & $0.00 \mathrm{E}+00$ & $0.00 \mathrm{E}+00$ & $0.00 \mathrm{E}+00$ \\
\hline $\ln -114 \mathrm{~m}$ & 7.06E-04 & $2.58 \mathrm{E}-07$ & $2.05 \mathrm{E}-05$ & $6.56 \mathrm{E}-04$ & $2.99 \mathrm{E}-05$ & $6.56 \mathrm{E}-04$ \\
\hline $\ln -115$ & $1.25 \mathrm{E}-02$ & $0.00 \mathrm{E}+00$ & $0.00 \mathrm{E}+00$ & 1.19E-02 & $5.60 \mathrm{E}-04$ & 1.19E-02 \\
\hline In-115m & $3.16 \mathrm{E}-06$ & $2.76 \mathrm{E}-07$ & $9.90 \mathrm{E}-07$ & $1.88 \mathrm{E}-06$ & 8.05E-09 & $2.16 \mathrm{E}-06$ \\
\hline In-116m & 4.00E-06 & $2.04 \mathrm{E}-06$ & $1.52 \mathrm{E}-06$ & 4.35E-07 & $4.00 \mathrm{E}-10$ & $2.48 \mathrm{E}-06$ \\
\hline $\ln -117$ & 7.71E-07 & 4.37E-07 & 3.34E-07 & $0.00 \mathrm{E}+00$ & $0.00 \mathrm{E}+00$ & 4.37E-07 \\
\hline In-117m & $1.59 \mathrm{E}-06$ & 4.93E-07 & 1.10E-06 & $0.00 \mathrm{E}+00$ & $0.00 \mathrm{E}+00$ & 4.93E-07 \\
\hline Sn-113 & $1.55 \mathrm{E}-04$ & $2.40 \mathrm{E}-07$ & 4.66E-05 & 1.04E-04 & 4.82E-06 & 1.04E-04 \\
\hline Sn-117m & $2.42 \mathrm{E}-05$ & $3.16 \mathrm{E}-07$ & $2.39 \mathrm{E}-05$ & $0.00 \mathrm{E}+00$ & $0.00 \mathrm{E}+00$ & $3.16 \mathrm{E}-07$ \\
\hline Sn-119m & 7.42E-05 & 4.60E-09 & 9.93E-07 & 7.00E-05 & $3.28 \mathrm{E}-06$ & $7.00 \mathrm{E}-05$ \\
\hline Sn-121 & 6.64E-06 & $0.00 \mathrm{E}+00$ & $0.00 \mathrm{E}+00$ & $6.52 \mathrm{E}-06$ & 1.19E-07 & $6.52 \mathrm{E}-06$ \\
\hline Sn-121m & 2.04E-04 & $0.00 \mathrm{E}+00$ & $0.00 \mathrm{E}+00$ & 1.95E-04 & 9.34E-06 & 1.95E-04 \\
\hline Sn-123 & 3.99E-04 & $1.52 \mathrm{E}-08$ & 1.07E-06 & $3.80 \mathrm{E}-04$ & 1.77E-05 & $3.80 \mathrm{E}-04$ \\
\hline Sn-125 & $3.88 \mathrm{E}-05$ & $6.78 \mathrm{E}-07$ & $3.81 \mathrm{E}-05$ & $4.90 \mathrm{E}-09$ & 8.84E-09 & $6.83 \mathrm{E}-07$ \\
\hline Sn-126 & 1.62E-03 & $3.29 \mathrm{E}-06$ & $2.91 \mathrm{E}-04$ & $1.26 \mathrm{E}-03$ & 5.96E-05 & $1.26 \mathrm{E}-03$ \\
\hline Sb-117 & 9.17E-07 & $2.69 \mathrm{E}-07$ & $6.48 \mathrm{E}-07$ & $0.00 \mathrm{E}+00$ & $0.00 \mathrm{E}+00$ & 2.69E-07 \\
\hline Sb-120b & 5.04E-05 & $0.00 \mathrm{E}+00$ & $0.00 \mathrm{E}+00$ & 4.86E-05 & $1.78 \mathrm{E}-06$ & 4.86E-05 \\
\hline Sb-122 & 1.00E-04 & $9.34 \mathrm{E}-07$ & $3.49 \mathrm{E}-05$ & $6.24 \mathrm{E}-05$ & $1.81 \mathrm{E}-06$ & $6.33 \mathrm{E}-05$ \\
\hline Sb-124 & 5.93E-04 & $4.18 \mathrm{E}-06$ & $2.83 \mathrm{E}-04$ & $2.93 E-04$ & $1.34 \mathrm{E}-05$ & $2.97 \mathrm{E}-04$ \\
\hline Sb-125 & $2.16 \mathrm{E}-04$ & $9.00 \mathrm{E}-07$ & 7.39E-05 & 1.34E-04 & $6.35 \mathrm{E}-06$ & 1.35E-04 \\
\hline Sb-126 & $5.55 \mathrm{E}-04$ & 5.94E-06 & 3.87E-04 & $1.55 \mathrm{E}-04$ & $6.47 \mathrm{E}-06$ & 1.61E-04 \\
\hline Sb-126m & $4.21 \mathrm{E}-07$ & $2.08 \mathrm{E}-07$ & $1.52 \mathrm{E}-07$ & $6.12 \mathrm{E}-08$ & $9.77 \mathrm{E}-10$ & $2.69 \mathrm{E}-07$ \\
\hline Sb-127 & $6.59 \mathrm{E}-05$ & 1.41E-06 & $6.45 \mathrm{E}-05$ & $0.00 \mathrm{E}+00$ & $0.00 \mathrm{E}+00$ & $1.41 \mathrm{E}-06$ \\
\hline Sb-129 & $2.02 \mathrm{E}-05$ & $2.61 \mathrm{E}-06$ & 7.96E-06 & $9.53 \mathrm{E}-06$ & 5.57E-08 & $1.21 \mathrm{E}-05$ \\
\hline Te-121 & 1.09E-04 & 1.19E-06 & 8.47E-05 & $2.19 \mathrm{E}-05$ & 9.44E-07 & 2.31E-05 \\
\hline Te-121m & 2.03E-04 & 4.46E-07 & $4.88 \mathrm{E}-05$ & 1.46E-04 & 6.91E-06 & 1.46E-04 \\
\hline Te-123 & 5.61E-05 & $6.38 \mathrm{E}-09$ & $1.18 \mathrm{E}-06$ & $5.25 \mathrm{E}-05$ & 2.47E-06 & $5.25 \mathrm{E}-05$ \\
\hline Te-123m & $7.28 \mathrm{E}-05$ & $3.02 \mathrm{E}-07$ & $2.66 \mathrm{E}-05$ & 4.39E-05 & 2.04E-06 & $4.42 \mathrm{E}-05$ \\
\hline Te-125m & 9.04E-05 & $2.02 \mathrm{E}-08$ & 3.34E-06 & 8.32E-05 & 3.82E-06 & 8.32E-05 \\
\hline Te-127 & 7.43E-08 & $9.30 \mathrm{E}-09$ & $6.50 \mathrm{E}-08$ & $0.00 \mathrm{E}+00$ & $0.00 \mathrm{E}+00$ & $9.30 \mathrm{E}-09$ \\
\hline Te-127m & $1.85 \mathrm{E}-06$ & 7.41E-09 & $1.84 \mathrm{E}-06$ & $0.00 \mathrm{E}+00$ & $0.00 \mathrm{E}+00$ & 7.41E-09 \\
\hline Te-129 & $8.55 \mathrm{E}-07$ & $5.36 \mathrm{E}-08$ & $5.74 \mathrm{E}-08$ & 7.43E-07 & $8.75 \mathrm{E}-10$ & 7.97E-07 \\
\hline Te-129m & 3.06E-04 & 1.12E-07 & 1.14E-05 & $2.82 \mathrm{E}-04$ & 1.27E-05 & $2.82 \mathrm{E}-04$ \\
\hline Te-131 & 1.43E-06 & 1.09E-07 & 7.36E-07 & 4.04E-07 & 1.82E-07 & $5.13 \mathrm{E}-07$ \\
\hline Te-131m & 1.11E-04 & $3.22 \mathrm{E}-06$ & 7.02E-05 & $3.53 E-05$ & 2.31E-06 & $3.85 \mathrm{E}-05$ \\
\hline Te-132 & $3.29 \mathrm{E}-04$ & $1.21 \mathrm{E}-06$ & $2.36 \mathrm{E}-04$ & $8.90 \mathrm{E}-05$ & $2.89 \mathrm{E}-06$ & $9.02 \mathrm{E}-05$ \\
\hline Te-133 & $1.77 \mathrm{E}-06$ & $3.38 \mathrm{E}-08$ & $1.27 \mathrm{E}-06$ & $3.78 \mathrm{E}-07$ & 8.92E-08 & $4.12 \mathrm{E}-07$ \\
\hline Te-133m & 8.96E-06 & $2.02 \mathrm{E}-06$ & $5.58 \mathrm{E}-06$ & $1.08 \mathrm{E}-06$ & $2.79 \mathrm{E}-07$ & $3.10 \mathrm{E}-06$ \\
\hline
\end{tabular}


Table 4. Ground release, Class F, $1 \mathrm{~m} / \mathrm{s}$ wind, $1 \mathrm{~km}$, no rain, continued.

\begin{tabular}{|c|c|c|c|c|c|c|}
\hline Nuclide & $\begin{array}{c}\text { 7-day } \\
\text { Total } \\
(1+2+3+4)\end{array}$ & $\begin{array}{c}\text { Cloud } \\
\text { shine } \\
(1)\end{array}$ & $\begin{array}{c}\text { Ground } \\
\text { shine } \\
(2)\end{array}$ & $\begin{array}{c}\text { Inhalation } \\
\text { (3) }\end{array}$ & $\begin{array}{c}\text { Resus- } \\
\text { pension } \\
(4)\end{array}$ & $\begin{array}{c}\text { Plume } \\
\text { Passage } \\
(1)+(3)\end{array}$ \\
\hline Te-134 & 1.33E-05 & 1.40E-06 & 1.16E-05 & $2.48 \mathrm{E}-07$ & 3.73E-09 & 1.65E-06 \\
\hline $\mid-122$ & $2.04 \mathrm{E}-12$ & $3.81 \mathrm{E}-13$ & $1.66 \mathrm{E}-12$ & $0.00 \mathrm{E}+00$ & $0.00 \mathrm{E}+00$ & $3.81 \mathrm{E}-13$ \\
\hline $\mid-123$ & 2.27E-05 & 1.39E-07 & $2.25 \mathrm{E}-05$ & $6.21 \mathrm{E}-13$ & $2.31 \mathrm{E}-12$ & 1.39E-07 \\
\hline $\mid-124$ & 6.86E-04 & $1.00 \mathrm{E}-06$ & $6.85 \mathrm{E}-04$ & $0.00 \mathrm{E}+00$ & $0.00 \mathrm{E}+00$ & 1.00E-06 \\
\hline $\mid-125$ & 2.65E-05 & 1.02E-08 & $2.65 \mathrm{E}-05$ & $0.00 \mathrm{E}+00$ & $0.00 \mathrm{E}+00$ & 1.02E-08 \\
\hline $\mid-126$ & 5.91E-04 & $4.28 \mathrm{E}-07$ & $4.44 \mathrm{E}-04$ & 8.90E-05 & 5.75E-05 & 8.94E-05 \\
\hline $\mid-128$ & 1.13E-07 & $8.28 \mathrm{E}-09$ & $6.93 \mathrm{E}-08$ & $3.52 \mathrm{E}-08$ & $2.32 \mathrm{E}-10$ & 4.35E-08 \\
\hline $\mid-129$ & 1.35E-03 & 7.61E-09 & $2.45 \mathrm{E}-05$ & 7.66E-04 & 5.55E-04 & 7.66E-04 \\
\hline $\mid-130$ & 2.65E-04 & $1.88 \mathrm{E}-06$ & $2.50 \mathrm{E}-04$ & $1.15 \mathrm{E}-05$ & 1.82E-06 & $1.34 \mathrm{E}-05$ \\
\hline $\mid-131$ & $5.85 \mathrm{E}-04$ & $3.52 \mathrm{E}-07$ & 3.41E-04 & $1.52 \mathrm{E}-04$ & $9.16 \mathrm{E}-05$ & 1.52E-04 \\
\hline $\mid-132$ & 4.27E-05 & $1.49 \mathrm{E}-06$ & 3.99E-05 & $1.22 \mathrm{E}-06$ & 4.29E-08 & 2.71E-06 \\
\hline $\mid-133$ & 1.73E-04 & $5.44 \mathrm{E}-07$ & $1.26 \mathrm{E}-04$ & 3.78E-05 & 8.90E-06 & 3.83E-05 \\
\hline $\mid-134$ & 1.23E-05 & $9.24 \mathrm{E}-07$ & 1.11E-05 & $2.62 \mathrm{E}-07$ & 3.61E-09 & 1.19E-06 \\
\hline $\mid-135$ & $1.18 \mathrm{E}-04$ & $1.50 \mathrm{E}-06$ & $1.04 \mathrm{E}-04$ & 1.10E-05 & $1.03 E-06$ & $1.25 \mathrm{E}-05$ \\
\hline Xe-122 & 6.47E-06 & $1.31 \mathrm{E}-06$ & $5.15 \mathrm{E}-06$ & $0.00 \mathrm{E}+00$ & $0.00 \mathrm{E}+00$ & $1.31 \mathrm{E}-06$ \\
\hline $\mathrm{Xe}-123$ & $2.30 \mathrm{E}-06$ & $9.44 \mathrm{E}-07$ & $1.35 \mathrm{E}-06$ & $1.78 \mathrm{E}-14$ & $1.38 \mathrm{E}-13$ & 9.44E-07 \\
\hline Xe-125 & 5.52E-07 & $5.35 \mathrm{E}-07$ & $1.70 \mathrm{E}-08$ & $0.00 \mathrm{E}+00$ & $0.00 \mathrm{E}+00$ & $5.35 \mathrm{E}-07$ \\
\hline Xe-127 & 5.94E-07 & 5.94E-07 & $0.00 \mathrm{E}+00$ & $0.00 \mathrm{E}+00$ & $0.00 \mathrm{E}+00$ & 5.94E-07 \\
\hline Xe-129m & $5.16 \mathrm{E}-08$ & $5.16 \mathrm{E}-08$ & $0.00 \mathrm{E}+00$ & $0.00 \mathrm{E}+00$ & $0.00 \mathrm{E}+00$ & $5.16 \mathrm{E}-08$ \\
\hline $\mathrm{Xe}-131 \mathrm{~m}$ & 1.91E-08 & 1.91E-08 & $0.00 \mathrm{E}+00$ & $0.00 \mathrm{E}+00$ & $0.00 \mathrm{E}+00$ & $1.91 \mathrm{E}-08$ \\
\hline Xe-133 & 7.78E-08 & $7.78 \mathrm{E}-08$ & $0.00 \mathrm{E}+00$ & $0.00 \mathrm{E}+00$ & $0.00 \mathrm{E}+00$ & $7.78 \mathrm{E}-08$ \\
\hline $\mathrm{Xe}-133 \mathrm{~m}$ & 6.61E-08 & $6.61 \mathrm{E}-08$ & $0.00 \mathrm{E}+00$ & $0.00 \mathrm{E}+00$ & $0.00 \mathrm{E}+00$ & $6.61 \mathrm{E}-08$ \\
\hline Xe-135 & 5.11E-07 & $5.11 \mathrm{E}-07$ & $0.00 \mathrm{E}+00$ & $2.03 \mathrm{E}-15$ & $9.54 \mathrm{E}-17$ & 5.11E-07 \\
\hline Xe-135m & 4.41E-08 & $4.41 \mathrm{E}-08$ & $0.00 \mathrm{E}+00$ & $4.14 \mathrm{E}-17$ & 1.95E-18 & 4.41E-08 \\
\hline Xe-138 & 1.14E-06 & 7.77E-07 & $3.61 \mathrm{E}-07$ & $0.00 \mathrm{E}+00$ & $0.00 \mathrm{E}+00$ & 7.77E-07 \\
\hline Cs-126 & $2.51 \mathrm{E}-20$ & $2.02 \mathrm{E}-20$ & $4.91 \mathrm{E}-21$ & $0.00 \mathrm{E}+00$ & $0.00 \mathrm{E}+00$ & $2.02 \mathrm{E}-20$ \\
\hline Cs-129 & 1.34E-05 & 5.34E-07 & $1.29 \mathrm{E}-05$ & $0.00 \mathrm{E}+00$ & $0.00 \mathrm{E}+00$ & 5.34E-07 \\
\hline Cs-131 & 2.59E-06 & 1.49E-08 & $2.58 \mathrm{E}-06$ & $0.00 \mathrm{E}+00$ & $0.00 \mathrm{E}+00$ & 1.49E-08 \\
\hline Cs-132 & 1.01E-04 & $1.50 \mathrm{E}-06$ & $8.50 \mathrm{E}-05$ & $1.38 \mathrm{E}-05$ & $5.18 \mathrm{E}-07$ & 1.53E-05 \\
\hline Cs-134 & 8.37E-04 & 3.37E-06 & $2.62 \mathrm{E}-04$ & $5.46 \mathrm{E}-04$ & $2.57 \mathrm{E}-05$ & $5.49 \mathrm{E}-04$ \\
\hline Cs-134m & 1.99E-07 & $3.22 \mathrm{E}-08$ & 1.39E-07 & $2.31 \mathrm{E}-08$ & 3.94E-09 & $5.53 \mathrm{E}-08$ \\
\hline Cs-135 & 5.05E-05 & $0.00 \mathrm{E}+00$ & $0.00 \mathrm{E}+00$ & 4.83E-05 & $2.27 \mathrm{E}-06$ & 4.83E-05 \\
\hline Cs-136 & 3.93E-04 & $4.72 \mathrm{E}-06$ & $2.99 \mathrm{E}-04$ & $8.55 \mathrm{E}-05$ & $3.59 \mathrm{E}-06$ & $9.02 \mathrm{E}-05$ \\
\hline Cs-137 & 5.09E-04 & $1.22 \mathrm{E}-06$ & $9.64 \mathrm{E}-05$ & 3.93E-04 & $1.85 \mathrm{E}-05$ & 3.94E-04 \\
\hline Cs-138 & $1.55 \mathrm{E}-06$ & 1.03E-06 & $5.23 \mathrm{E}-07$ & $0.00 \mathrm{E}+00$ & $0.00 \mathrm{E}+00$ & 1.03E-06 \\
\hline Ba-131 & 6.93E-05 & $9.60 \mathrm{E}-07$ & $6.83 \mathrm{E}-05$ & $0.00 \mathrm{E}+00$ & $0.00 \mathrm{E}+00$ & $9.60 \mathrm{E}-07$ \\
\hline Ba-133 & 1.54E-04 & 7.73E-07 & $6.98 \mathrm{E}-05$ & 7.97E-05 & $3.75 \mathrm{E}-06$ & $8.05 \mathrm{E}-05$ \\
\hline Ba-133m & 1.06E-05 & 1.16E-07 & $3.66 \mathrm{E}-06$ & $6.71 \mathrm{E}-06$ & $1.53 \mathrm{E}-07$ & $6.83 \mathrm{E}-06$ \\
\hline Ba-135m & 2.59E-06 & $1.02 \mathrm{E}-07$ & $2.48 \mathrm{E}-06$ & $0.00 \mathrm{E}+00$ & $0.00 \mathrm{E}+00$ & $1.02 \mathrm{E}-07$ \\
\hline Ba-137m & $1.44 \mathrm{E}-15$ & 1.17E-15 & $2.75 \mathrm{E}-16$ & $0.00 \mathrm{E}+00$ & $0.00 \mathrm{E}+00$ & 1.17E-15 \\
\hline Ba-139 & 1.29E-06 & $3.98 \mathrm{E}-08$ & $5.05 \mathrm{E}-08$ & 1.19E-06 & 1.66E-09 & 1.23E-06 \\
\hline
\end{tabular}


Table 4. Ground release, Class F, $1 \mathrm{~m} / \mathrm{s}$ wind, $1 \mathrm{~km}$, no rain, continued.

\begin{tabular}{|c|c|c|c|c|c|c|}
\hline Nuclide & $\begin{array}{c}\text { 7-day } \\
\text { Total } \\
(1+2+3+4)\end{array}$ & $\begin{array}{c}\text { Cloud } \\
\text { shine } \\
(1)\end{array}$ & $\begin{array}{c}\text { Ground } \\
\text { shine } \\
(2)\end{array}$ & $\begin{array}{c}\text { Inhalation } \\
\text { (3) }\end{array}$ & $\begin{array}{c}\text { Resus- } \\
\text { pension } \\
(4)\end{array}$ & $\begin{array}{c}\text { Plume } \\
\text { Passage } \\
(1)+(3)\end{array}$ \\
\hline Ba-140 & 2.83E-04 & $5.08 \mathrm{E}-07$ & $2.35 \mathrm{E}-04$ & 4.42E-05 & $3.16 \mathrm{E}-06$ & 4.47E-05 \\
\hline Ba-141 & $1.85 \mathrm{E}-07$ & 1.11E-07 & $6.62 \mathrm{E}-08$ & 5.69E-09 & 1.71E-09 & 1.17E-07 \\
\hline Ba-142 & 9.73E-07 & $4.88 \mathrm{E}-07$ & $4.85 \mathrm{E}-07$ & $0.00 \mathrm{E}+00$ & $0.00 \mathrm{E}+00$ & $4.88 \mathrm{E}-07$ \\
\hline La-137 & $2.69 \mathrm{E}-04$ & $0.00 \mathrm{E}+00$ & $0.00 \mathrm{E}+00$ & 2.57E-04 & $1.21 \mathrm{E}-05$ & 2.57E-04 \\
\hline La-138 & 4.26E-03 & $0.00 \mathrm{E}+00$ & $0.00 \mathrm{E}+00$ & 4.07E-03 & 1.92E-04 & 4.07E-03 \\
\hline La-140 & 1.82E-04 & 5.04E-06 & $1.15 \mathrm{E}-04$ & 6.06E-05 & 1.40E-06 & 6.56E-05 \\
\hline La-141 & 4.55E-07 & 7.47E-08 & $2.52 \mathrm{E}-07$ & 1.06E-07 & $2.22 \mathrm{E}-08$ & $1.81 \mathrm{E}-07$ \\
\hline La-142 & 7.44E-06 & $3.71 \mathrm{E}-06$ & $3.72 \mathrm{E}-06$ & $0.00 \mathrm{E}+00$ & $0.00 \mathrm{E}+00$ & 3.71E-06 \\
\hline Ce-139 & 1.32E-04 & $3.15 \mathrm{E}-07$ & $2.88 \mathrm{E}-05$ & $9.85 \mathrm{E}-05$ & 4.59E-06 & $9.88 \mathrm{E}-05$ \\
\hline Ce-141 & 1.23E-04 & $1.62 \mathrm{E}-07$ & $1.36 \mathrm{E}-05$ & 1.05E-04 & 4.70E-06 & 1.05E-04 \\
\hline Ce-143 & 1.34E-05 & 5.39E-07 & $1.29 \mathrm{E}-05$ & $0.00 \mathrm{E}+00$ & $0.00 \mathrm{E}+00$ & 5.39E-07 \\
\hline Ce-144 & $4.50 \mathrm{E}-03$ & $1.10 \mathrm{E}-07$ & $8.51 \mathrm{E}-06$ & 4.29E-03 & 2.01E-04 & 4.29E-03 \\
\hline Pr-142 & 3.96E-05 & $1.25 \mathrm{E}-07$ & $1.36 \mathrm{E}-06$ & 3.76E-05 & 5.41E-07 & 3.77E-05 \\
\hline Pr-143 & $1.29 \mathrm{E}-12$ & $1.94 \mathrm{E}-14$ & $1.27 \mathrm{E}-12$ & $0.00 \mathrm{E}+00$ & $0.00 \mathrm{E}+00$ & $1.94 \mathrm{E}-14$ \\
\hline $\mathrm{Pr}-144$ & 4.73E-09 & 3.47E-09 & 1.25E-09 & $0.00 \mathrm{E}+00$ & $0.00 \mathrm{E}+00$ & 3.47E-09 \\
\hline Pr-144m & 3.34E-09 & $2.45 \mathrm{E}-09$ & $8.91 \mathrm{E}-10$ & $0.00 \mathrm{E}+00$ & $0.00 \mathrm{E}+00$ & 2.45E-09 \\
\hline $\mathrm{Nd}-141$ & 1.07E-07 & $0.00 \mathrm{E}+00$ & $0.00 \mathrm{E}+00$ & 1.07E-07 & $2.64 \mathrm{E}-10$ & 78-07 \\
\hline Nd-147 & 1.04E-04 & 2.79E-07 & $2.00 \mathrm{E}-05$ & 8.02E-05 & 3.33E-06 & 8.05E-05 \\
\hline Nd-149 & 3.96E-06 & $4.81 \mathrm{E}-07$ & $7.60 \mathrm{E}-07$ & $2.68 \mathrm{E}-06$ & $3.47 \mathrm{E}-08$ & $.16 \mathrm{E}-06$ \\
\hline $\mathrm{Pm}-143$ & 1.75E-04 & $6.51 \mathrm{E}-07$ & $5.19 \mathrm{E}-05$ & 1.17E-04 & $5.48 \mathrm{E}-06$ & $1.18 \mathrm{E}-04$ \\
\hline $\mathrm{Pm}-144$ & 2.66E-04 & $3.31 \mathrm{E}-06$ & $2.63 \mathrm{E}-04$ & $0.00 \mathrm{E}+00$ & $0.00 \mathrm{E}+00$ & 3.31E-06 \\
\hline $\mathrm{Pm}-145$ & 2.99E-04 & $3.47 \mathrm{E}-08$ & 4.79E-06 & $2.81 \mathrm{E}-04$ & $1.32 \mathrm{E}-05$ & $2.81 \mathrm{E}-04$ \\
\hline $\mathrm{Pm}-146$ & 1.86E-03 & $1.60 \mathrm{E}-06$ & $1.28 \mathrm{E}-04$ & 1.65E-03 & $7.78 \mathrm{E}-05$ & $1.65 \mathrm{E}-03$ \\
\hline $\mathrm{Pm}-147$ & 4.75E-04 & $7.65 \mathrm{E}-12$ & $6.78 \mathrm{E}-10$ & 4.53E-04 & $2.13 \mathrm{E}-05$ & 4.53E-04 \\
\hline $\mathrm{Pm}-148$ & $6.00 \mathrm{E}-05$ & $1.24 \mathrm{E}-06$ & $5.87 \mathrm{E}-05$ & $0.00 \mathrm{E}+00$ & $0.00 \mathrm{E}+00$ & $1.24 \mathrm{E}-06$ \\
\hline $\mathrm{Pm}-148 \mathrm{~m}$ & 3.26E-04 & $4.31 \mathrm{E}-06$ & $3.22 \mathrm{E}-04$ & $0.00 \mathrm{E}+00$ & $0.00 \mathrm{E}+00$ & $4.31 \mathrm{E}-06$ \\
\hline $\mathrm{Pm}-149$ & $3.78 \mathrm{E}-05$ & $2.43 \mathrm{E}-08$ & $8.33 \mathrm{E}-07$ & $3.60 \mathrm{E}-05$ & $9.55 \mathrm{E}-07$ & $3.60 \mathrm{E}-05$ \\
\hline $\mathrm{Pm}-151$ & $1.48 \mathrm{E}-05$ & $6.84 \mathrm{E}-07$ & $1.41 \mathrm{E}-05$ & $3.89 \mathrm{E}-10$ & $3.65 \mathrm{E}-10$ & $6.84 \mathrm{E}-07$ \\
\hline Sm-146 & $1.00 \mathrm{E}+00$ & $0.00 \mathrm{E}+00$ & $0.00 \mathrm{E}+00$ & $9.59 \mathrm{E}-01$ & $4.52 \mathrm{E}-02$ & $9.59 \mathrm{E}-01$ \\
\hline Sm-147 & $9.12 \mathrm{E}-01$ & $0.00 \mathrm{E}+00$ & $0.00 \mathrm{E}+00$ & $8.71 \mathrm{E}-01$ & $4.10 \mathrm{E}-02$ & 8.71E-01 \\
\hline Sm-151 & 3.68E-04 & 1.93E-12 & $8.70 \mathrm{E}-10$ & $3.52 \mathrm{E}-04$ & 1.66E-05 & $3.52 \mathrm{E}-04$ \\
\hline Sm-153 & 2.89E-05 & 1.05E-07 & 4.26E-06 & 2.39E-05 & 5.97E-07 & $2.40 \mathrm{E}-05$ \\
\hline Eu-150b & 3.17E-03 & $0.00 \mathrm{E}+00$ & $0.00 \mathrm{E}+00$ & $3.02 \mathrm{E}-03$ & $1.42 \mathrm{E}-04$ & $3.02 \mathrm{E}-03$ \\
\hline Eu-152 & 2.77E-03 & $2.48 \mathrm{E}-06$ & $1.86 \mathrm{E}-04$ & $2.46 \mathrm{E}-03$ & 1.16E-04 & $2.46 \mathrm{E}-03$ \\
\hline Eu-152m & $1.24 \mathrm{E}-05$ & $6.22 \mathrm{E}-07$ & $3.97 \mathrm{E}-06$ & 7.71E-06 & $6.29 \mathrm{E}-08$ & 8.33E-06 \\
\hline Eu-154 & 3.61E-03 & $2.73 \mathrm{E}-06$ & 2.01E-04 & $3.25 \mathrm{E}-03$ & 1.53E-04 & $3.25 \mathrm{E}-03$ \\
\hline Eu-155 & 1.19E-05 & $1.20 \mathrm{E}-07$ & $1.18 \mathrm{E}-05$ & $0.00 \mathrm{E}+00$ & $0.00 \mathrm{E}+00$ & $1.20 \mathrm{E}-07$ \\
\hline Eu-156 & 1.82E-04 & $3.08 \mathrm{E}-06$ & 1.79E-04 & $0.00 \mathrm{E}+00$ & $0.00 \mathrm{E}+00$ & $3.08 \mathrm{E}-06$ \\
\hline Gd-148 & $1.06 \mathrm{E}+00$ & $0.00 \mathrm{E}+00$ & $0.00 \mathrm{E}+00$ & $1.01 \mathrm{E}+00$ & $4.77 \mathrm{E}-02$ & $1.01 \mathrm{E}+00$ \\
\hline Gd-152 & 7.93E-01 & $0.00 \mathrm{E}+00$ & $0.00 \mathrm{E}+00$ & 7.57E-01 & 3.57E-02 & 7.57E-01 \\
\hline Gd-153 & 2.09E-05 & 1.86E-07 & $2.07 E-05$ & $0.00 \mathrm{E}+00$ & $0.00 \mathrm{E}+00$ & $1.86 \mathrm{E}-07$ \\
\hline
\end{tabular}


Table 4. Ground release, Class F, $1 \mathrm{~m} / \mathrm{s}$ wind, $1 \mathrm{~km}$, no rain, continued.

\begin{tabular}{|c|c|c|c|c|c|c|}
\hline Nuclide & $\begin{array}{c}\text { 7-day } \\
\text { Total } \\
(1+2+3+4)\end{array}$ & $\begin{array}{c}\text { Cloud } \\
\text { shine } \\
(1)\end{array}$ & $\begin{array}{c}\text { Ground } \\
\text { shine } \\
(2)\end{array}$ & $\begin{array}{c}\text { Inhalation } \\
\text { (3) }\end{array}$ & $\begin{array}{c}\text { Resus- } \\
\text { pension } \\
(4)\end{array}$ & $\begin{array}{c}\text { Plume } \\
\text { Passage } \\
(1)+(3)\end{array}$ \\
\hline Gd-159 & $1.44 \mathrm{E}-05$ & 7.53E-08 & $1.12 \mathrm{E}-06$ & $1.31 \mathrm{E}-05$ & 1.84E-07 & 1.32E-05 \\
\hline Tb-157 & 7.82E-05 & 5.37E-09 & $7.70 \mathrm{E}-07$ & $7.40 \mathrm{E}-05$ & $3.48 \mathrm{E}-06$ & 7.40E-05 \\
\hline Tb-158 & $2.75 \mathrm{E}-03$ & $0.00 \mathrm{E}+00$ & $0.00 \mathrm{E}+00$ & 2.63E-03 & $1.24 \mathrm{E}-04$ & 2.63E-03 \\
\hline Tb-160 & $4.42 \mathrm{E}-04$ & $2.36 \mathrm{E}-06$ & 1.70E-04 & $2.58 \mathrm{E}-04$ & 1.19E-05 & $2.60 \mathrm{E}-04$ \\
\hline $\mathrm{Tb}-161$ & $4.18 \mathrm{E}-05$ & $0.00 \mathrm{E}+00$ & $0.00 \mathrm{E}+00$ & 4.02E-05 & $1.53 \mathrm{E}-06$ & $4.02 \mathrm{E}-05$ \\
\hline Dy-157 & 4.76E-06 & $6.40 \mathrm{E}-07$ & $4.12 \mathrm{E}-06$ & $4.72 \mathrm{E}-11$ & $1.85 \mathrm{E}-11$ & $6.40 \mathrm{E}-07$ \\
\hline Dy-159 & $2.41 \mathrm{E}-05$ & $0.00 \mathrm{E}+00$ & $0.00 \mathrm{E}+00$ & $2.30 \mathrm{E}-05$ & 1.07E-06 & $2.30 \mathrm{E}-05$ \\
\hline Dy-165 & 1.07E-07 & $3.56 \mathrm{E}-08$ & 7.19E-08 & $0.00 \mathrm{E}+00$ & $0.00 \mathrm{E}+00$ & 3.56E-08 \\
\hline Dy-166 & 4.76E-04 & $6.75 \mathrm{E}-08$ & $6.04 \mathrm{E}-06$ & $2.88 \mathrm{E}-04$ & 1.82E-04 & $2.88 \mathrm{E}-04$ \\
\hline Ho-164 & $3.13 \mathrm{E}-08$ & $0.00 \mathrm{E}+00$ & $0.00 \mathrm{E}+00$ & $3.13 \mathrm{E}-08$ & $1.56 \mathrm{E}-11$ & 3.13E-08 \\
\hline Ho-164m & 1.65E-07 & $0.00 \mathrm{E}+00$ & $0.00 \mathrm{E}+00$ & 1.65E-07 & $1.26 \mathrm{E}-10$ & 1.65E-07 \\
\hline Ho-166 & $8.78 \mathrm{E}-03$ & $5.72 \mathrm{E}-08$ & 1.06E-06 & $8.62 \mathrm{E}-03$ & 1.57E-04 & $8.62 \mathrm{E}-03$ \\
\hline Ho-166m & $2.78 \mathrm{E}-04$ & $3.46 \mathrm{E}-06$ & $2.75 \mathrm{E}-04$ & $0.00 \mathrm{E}+00$ & $0.00 \mathrm{E}+00$ & $3.46 \mathrm{E}-06$ \\
\hline Er-169 & $2.55 \mathrm{E}-05$ & $4.00 \mathrm{E}-12$ & $6.44 \mathrm{E}-10$ & $2.45 \mathrm{E}-05$ & $9.85 \mathrm{E}-07$ & $2.45 \mathrm{E}-05$ \\
\hline Er-171 & $1.24 \mathrm{E}-05$ & $6.91 \mathrm{E}-07$ & 4.09E-06 & 7.56E-06 & $5.33 \mathrm{E}-08$ & $8.25 \mathrm{E}-06$ \\
\hline Tm-170 & 3.19E-04 & $9.71 \mathrm{E}-09$ & 1.02E-06 & 3.04E-04 & 1.42E-05 & 3.04E-04 \\
\hline $\mathrm{Tm}-171$ & 1.12E-04 & 1.10E-09 & $1.30 \mathrm{E}-07$ & 1.07E-04 & $5.01 \mathrm{E}-06$ & 1.07E-04 \\
\hline Yb-169 & 5.54E-05 & 5.99E-07 & $5.48 \mathrm{E}-05$ & $0.00 \mathrm{E}+00$ & $0.00 \mathrm{E}+00$ & 5.99E-07 \\
\hline Yb-175 & 4.27E-06 & $8.17 \mathrm{E}-08$ & $4.18 \mathrm{E}-06$ & $0.00 \mathrm{E}+00$ & $0.00 \mathrm{E}+00$ & 8.17E-08 \\
\hline Lu-174 & 4.61E-04 & $0.00 \mathrm{E}+00$ & $0.00 \mathrm{E}+00$ & 4.40E-04 & 2.07E-05 & $4.40 \mathrm{E}-04$ \\
\hline Lu-174m & 2.91E-04 & $0.00 \mathrm{E}+00$ & $0.00 \mathrm{E}+00$ & $2.78 \mathrm{E}-04$ & 1.30E-05 & $2.78 \mathrm{E}-04$ \\
\hline Lu-176 & 8.10E-03 & $0.00 \mathrm{E}+00$ & $0.00 \mathrm{E}+00$ & 7.74E-03 & $3.65 \mathrm{E}-04$ & 7.74E-03 \\
\hline Lu-176m & $3.78 \mathrm{E}-06$ & $0.00 \mathrm{E}+00$ & $0.00 \mathrm{E}+00$ & 3.77E-06 & $1.34 \mathrm{E}-08$ & 3.77E-06 \\
\hline Lu-177 & $3.65 \mathrm{E}-05$ & 7.31E-08 & $4.68 \mathrm{E}-06$ & 3.06E-05 & 1.16E-06 & 3.07E-05 \\
\hline Lu-177m & 5.97E-04 & $2.08 \mathrm{E}-06$ & $1.80 \mathrm{E}-04$ & 3.96E-04 & 1.86E-05 & $3.98 \mathrm{E}-04$ \\
\hline Lu-178 & 1.57E-07 & $0.00 \mathrm{E}+00$ & $0.00 \mathrm{E}+00$ & 1.57E-07 & 7.66E-11 & 1.57E-07 \\
\hline Lu-178m & 7.03E-08 & $0.00 \mathrm{E}+00$ & $0.00 \mathrm{E}+00$ & $7.02 \mathrm{E}-08$ & $2.74 \mathrm{E}-11$ & $7.02 \mathrm{E}-08$ \\
\hline $\mathrm{Hf}-175$ & $4.18 \mathrm{E}-05$ & $0.00 \mathrm{E}+00$ & $0.00 \mathrm{E}+00$ & 3.99E-05 & 1.84E-06 & 3.99E-05 \\
\hline $\mathrm{Hf}-177 \mathrm{~m}$ & 5.18E-07 & $0.00 \mathrm{E}+00$ & $0.00 \mathrm{E}+00$ & $5.17 \mathrm{E}-07$ & $4.52 \mathrm{E}-10$ & 5.17E-07 \\
\hline $\mathrm{Hf}-178 \mathrm{~m}$ & $6.43 \mathrm{E}-03$ & $0.00 \mathrm{E}+00$ & $0.00 \mathrm{E}+00$ & $6.14 \mathrm{E}-03$ & $2.89 \mathrm{E}-04$ & $6.14 \mathrm{E}-03$ \\
\hline $\mathrm{Hf}-179 \mathrm{~m}$ & 1.16E-04 & $0.00 \mathrm{E}+00$ & $0.00 \mathrm{E}+00$ & 1.11E-04 & 4.92E-06 & 1.11E-04 \\
\hline Hf-181 & 2.31E-04 & 1.14E-06 & $9.09 \mathrm{E}-05$ & 1.33E-04 & $6.03 \mathrm{E}-06$ & 1.34E-04 \\
\hline $\mathrm{Hf}-182$ & 1.05E-02 & $9.01 \mathrm{E}-10$ & $4.29 \mathrm{E}-06$ & $1.00 \mathrm{E}-02$ & $4.72 \mathrm{E}-04$ & 1.00E-02 \\
\hline $\mathrm{Hf}-183$ & 1.13E-06 & $0.00 \mathrm{E}+00$ & $0.00 \mathrm{E}+00$ & $1.11 \mathrm{E}-06$ & $1.85 \mathrm{E}-08$ & 1.11E-06 \\
\hline Тa-179 & 5.99E-05 & $0.00 \mathrm{E}+00$ & $0.00 \mathrm{E}+00$ & $5.72 \mathrm{E}-05$ & $2.69 \mathrm{E}-06$ & $5.72 \mathrm{E}-05$ \\
\hline Ta-180m & $9.88 \mathrm{E}-07$ & $0.00 \mathrm{E}+00$ & $0.00 \mathrm{E}+00$ & $9.81 \mathrm{E}-07$ & 7.11E-09 & $9.81 \mathrm{E}-07$ \\
\hline Ta-182 & $7.27 \mathrm{E}-04$ & $2.81 \mathrm{E}-06$ & $2.00 \mathrm{E}-04$ & 5.01E-04 & $2.33 \mathrm{E}-05$ & $5.04 \mathrm{E}-04$ \\
\hline Ta-182m & $7.60 \mathrm{E}-08$ & $2.59 \mathrm{E}-10$ & 1.91E-08 & $5.44 \mathrm{E}-08$ & 2.23E-09 & 5.47E-08 \\
\hline Ta-183 & $5.89 \mathrm{E}-05$ & $0.00 \mathrm{E}+00$ & $0.00 \mathrm{E}+00$ & 5.69E-05 & $2.03 \mathrm{E}-06$ & $5.69 \mathrm{E}-05$ \\
\hline Ta-184 & $1.27 \mathrm{E}-05$ & $0.00 \mathrm{E}+00$ & $0.00 \mathrm{E}+00$ & $1.26 \mathrm{E}-05$ & $9.70 \mathrm{E}-08$ & 1.26E-05 \\
\hline Ta-185 & 5.94E-07 & $1.75 \mathrm{E}-14$ & $2.26 \mathrm{E}-12$ & 5.94E-07 & $6.96 \mathrm{E}-10$ & 5.94E-07 \\
\hline
\end{tabular}


Table 4. Ground release, Class F, $1 \mathrm{~m} / \mathrm{s}$ wind, $1 \mathrm{~km}$, no rain, continued.

\begin{tabular}{|c|c|c|c|c|c|c|}
\hline Nuclide & $\begin{array}{c}\text { 7-day } \\
\text { Total } \\
(1+2+3+4)\end{array}$ & $\begin{array}{c}\text { Cloud } \\
\text { shine } \\
(1)\end{array}$ & $\begin{array}{c}\text { Ground } \\
\text { shine } \\
(2)\end{array}$ & $\begin{array}{c}\text { Inhalation } \\
\text { (3) }\end{array}$ & $\begin{array}{c}\text { Resus- } \\
\text { pension } \\
(4)\end{array}$ & $\begin{array}{c}\text { Plume } \\
\text { Passage } \\
(1)+(3)\end{array}$ \\
\hline Ta-186 & 2.93E-09 & $0.00 \mathrm{E}+00$ & $0.00 \mathrm{E}+00$ & 2.93E-09 & $5.30 \mathrm{E}-13$ & 2.93E-09 \\
\hline W-179 & 1.21E-08 & $0.00 \mathrm{E}+00$ & $0.00 \mathrm{E}+00$ & $1.20 \mathrm{E}-08$ & $1.12 \mathrm{E}-10$ & $1.20 \mathrm{E}-08$ \\
\hline W-181 & $9.00 \mathrm{E}-06$ & $6.84 \mathrm{E}-08$ & $7.55 \mathrm{E}-06$ & $1.32 \mathrm{E}-06$ & $6.14 \mathrm{E}-08$ & 1.39E-06 \\
\hline W-185 & 1.02E-05 & $5.83 \mathrm{E}-11$ & 5.01E-09 & 9.76E-06 & $4.50 \mathrm{E}-07$ & $9.76 \mathrm{E}-06$ \\
\hline W-187 & $2.46 \mathrm{E}-05$ & $9.81 \mathrm{E}-07$ & 1.65E-05 & 7.01E-06 & $1.18 \mathrm{E}-07$ & 7.99E-06 \\
\hline W-188 & 6.87E-05 & $1.01 \mathrm{E}-08$ & $8.91 \mathrm{E}-06$ & 5.64E-05 & $3.44 \mathrm{E}-06$ & 5.64E-05 \\
\hline $\mathrm{Re}-182 \mathrm{~b}$ & 1.35E-04 & $3.70 \mathrm{E}-06$ & $1.32 \mathrm{E}-04$ & $0.00 \mathrm{E}+00$ & $0.00 \mathrm{E}+00$ & 3.70E-06 \\
\hline $\mathrm{Re}-182 \mathrm{a}$ & 2.24E-05 & $2.42 \mathrm{E}-06$ & $2.00 \mathrm{E}-05$ & $0.00 \mathrm{E}+00$ & $0.00 \mathrm{E}+00$ & $2.42 \mathrm{E}-06$ \\
\hline Re-184 & $2.03 \mathrm{E}-04$ & 1.93E-06 & 1.42E-04 & $5.70 \mathrm{E}-05$ & $2.58 \mathrm{E}-06$ & 5.89E-05 \\
\hline $\mathrm{Re}-184 \mathrm{~m}$ & 1.81E-04 & $8.25 \mathrm{E}-07$ & $7.40 \mathrm{E}-05$ & 1.02E-04 & 4.82E-06 & 1.03E-04 \\
\hline Re-186 & 3.94E-05 & $4.23 \mathrm{E}-08$ & $2.23 \mathrm{E}-06$ & $3.60 \mathrm{E}-05$ & $1.18 \mathrm{E}-06$ & $3.60 \mathrm{E}-05$ \\
\hline Re-186m & $3.43 \mathrm{E}-04$ & $4.15 \mathrm{E}-10$ & $1.75 \mathrm{E}-06$ & $3.26 \mathrm{E}-04$ & 1.59E-05 & $3.26 \mathrm{E}-04$ \\
\hline Re-187 & $6.52 \mathrm{E}-07$ & $0.00 \mathrm{E}+00$ & $0.00 \mathrm{E}+00$ & $6.23 \mathrm{E}-07$ & 2.93E-08 & $6.23 \mathrm{E}-07$ \\
\hline Re-188 & $2.72 \mathrm{E}-05$ & 1.19E-07 & $1.46 \mathrm{E}-06$ & $2.53 \mathrm{E}-05$ & 3.34E-07 & $2.54 \mathrm{E}-05$ \\
\hline Re-188m & 5.09E-07 & 2.07E-09 & $2.71 \mathrm{E}-08$ & 4.73E-07 & $6.21 \mathrm{E}-09$ & $4.75 \mathrm{E}-07$ \\
\hline Re-189 & $1.44 \mathrm{E}-05$ & $0.00 \mathrm{E}+00$ & $0.00 \mathrm{E}+00$ & $1.41 \mathrm{E}-05$ & 2.42E-07 & 1.41E-05 \\
\hline Os-185 & 1.20E-04 & $1.51 \mathrm{E}-06$ & $1.18 \mathrm{E}-04$ & $0.00 \mathrm{E}+00$ & $0.00 \mathrm{E}+00$ & $1.51 \mathrm{E}-06$ \\
\hline Os-189m & 3.54E-07 & $0.00 \mathrm{E}+00$ & $0.00 \mathrm{E}+00$ & $3.52 \mathrm{E}-07$ & 1.96E-09 & $3.52 \mathrm{E}-07$ \\
\hline Os-190m & 2.14E-08 & 1.57E-08 & 5.71E-09 & $0.00 \mathrm{E}+00$ & $0.00 \mathrm{E}+00$ & 1.57E-08 \\
\hline Os-191 & 5.85E-05 & 1.45E-07 & $1.21 \mathrm{E}-05$ & 4.44E-05 & 1.90E-06 & 4.45E-05 \\
\hline Os-191m & 6.57E-07 & 8.39E-09 & 4.92E-07 & 1.03E-07 & $5.30 \mathrm{E}-08$ & $1.11 \mathrm{E}-07$ \\
\hline Os-193 & $3.16 \mathrm{E}-06$ & 1.36E-07 & $3.02 \mathrm{E}-06$ & $0.00 \mathrm{E}+00$ & $0.00 \mathrm{E}+00$ & 1.36E-07 \\
\hline Os-194 & 9.95E-03 & 8.82E-09 & $1.31 \mathrm{E}-05$ & $9.49 \mathrm{E}-03$ & $4.48 \mathrm{E}-04$ & $9.49 \mathrm{E}-03$ \\
\hline $\mid r-190$ & $2.66 \mathrm{E}-04$ & 2.96E-06 & $2.01 \mathrm{E}-04$ & 5.97E-05 & $2.48 \mathrm{E}-06$ & $6.27 \mathrm{E}-05$ \\
\hline Ir-190m & $1.23 \mathrm{E}-06$ & $4.84 \mathrm{E}-08$ & $8.88 \mathrm{E}-07$ & $2.88 \mathrm{E}-07$ & 1.04E-08 & 3.36E-07 \\
\hline Ir-190n & 1.30E-07 & $2.08 \mathrm{E}-09$ & 1.17E-07 & $9.84 \mathrm{E}-09$ & $1.28 \mathrm{E}-09$ & $1.19 \mathrm{E}-08$ \\
\hline Ir-192 & 4.81E-04 & $1.73 \mathrm{E}-06$ & 1.40E-04 & $3.24 \mathrm{E}-04$ & 1.49E-05 & $3.26 \mathrm{E}-04$ \\
\hline Ir-192m & 6.83E-03 & $8.63 \mathrm{E}-10$ & 4.70E-06 & $6.51 \mathrm{E}-03$ & 3.07E-04 & $6.51 \mathrm{E}-03$ \\
\hline Ir-194 & 4.08E-05 & 1.86E-07 & $2.50 \mathrm{E}-06$ & 3.76E-05 & $5.41 \mathrm{E}-07$ & $3.78 \mathrm{E}-05$ \\
\hline Ir-194m & 1.07E-03 & $4.98 \mathrm{E}-06$ & $4.01 \mathrm{E}-04$ & $6.38 \mathrm{E}-04$ & $2.98 \mathrm{E}-05$ & $6.43 \mathrm{E}-04$ \\
\hline Pt-191 & $2.55 \mathrm{E}-05$ & $5.84 \mathrm{E}-07$ & $2.50 \mathrm{E}-05$ & $0.00 \mathrm{E}+00$ & $0.00 \mathrm{E}+00$ & 5.84E-07 \\
\hline Pt-193 & 6.96E-07 & $9.29 \mathrm{E}-11$ & $9.34 \mathrm{E}-08$ & $5.75 \mathrm{E}-07$ & $2.71 \mathrm{E}-08$ & $5.75 \mathrm{E}-07$ \\
\hline Pt-193m & 5.51E-06 & $2.02 \mathrm{E}-08$ & $1.30 \mathrm{E}-06$ & 4.05E-06 & $1.38 \mathrm{E}-07$ & 4.07E-06 \\
\hline Pt-195m & 8.21E-06 & 1.36E-07 & $8.08 \mathrm{E}-06$ & $0.00 \mathrm{E}+00$ & $0.00 \mathrm{E}+00$ & 1.36E-07 \\
\hline Pt-197 & $6.72 \mathrm{E}-06$ & $4.55 \mathrm{E}-08$ & $6.95 \mathrm{E}-07$ & $5.90 \mathrm{E}-06$ & $8.23 \mathrm{E}-08$ & 5.95E-06 \\
\hline Pt-197m & 1.31E-06 & $9.41 \mathrm{E}-08$ & 2.02E-07 & $1.01 \mathrm{E}-06$ & 8.23E-09 & 1.10E-06 \\
\hline Au-194 & $6.81 \mathrm{E}-05$ & $2.34 \mathrm{E}-06$ & $5.53 E-05$ & 1.02E-05 & 2.33E-07 & $1.25 \mathrm{E}-05$ \\
\hline Au-195 & 1.62E-05 & $1.60 \mathrm{E}-07$ & 1.61E-05 & $0.00 \mathrm{E}+00$ & $0.00 \mathrm{E}+00$ & $1.60 \mathrm{E}-07$ \\
\hline Au-195m & $3.13 \mathrm{E}-11$ & $3.08 \mathrm{E}-13$ & $3.10 \mathrm{E}-11$ & $0.00 \mathrm{E}+00$ & $0.00 \mathrm{E}+00$ & $3.08 \mathrm{E}-13$ \\
\hline Au-198 & $8.58 \mathrm{E}-05$ & $8.42 \mathrm{E}-07$ & $3.28 \mathrm{E}-05$ & 5.07E-05 & 1.47E-06 & $5.15 \mathrm{E}-05$ \\
\hline Au-198m & $8.25 \mathrm{E}-05$ & $1.15 \mathrm{E}-08$ & 1.96E-05 & 6.06E-05 & $2.20 \mathrm{E}-06$ & $6.06 \mathrm{E}-05$ \\
\hline
\end{tabular}


Table 4. Ground release, Class F, $1 \mathrm{~m} / \mathrm{s}$ wind, $1 \mathrm{~km}$, no rain, continued.

\begin{tabular}{|c|c|c|c|c|c|c|}
\hline Nuclide & $\begin{array}{c}\text { 7-day } \\
\text { Total } \\
(1+2+3+4)\end{array}$ & $\begin{array}{c}\text { Cloud } \\
\text { shine } \\
(1)\end{array}$ & $\begin{array}{c}\text { Ground } \\
\text { shine } \\
(2)\end{array}$ & $\begin{array}{c}\text { Inhalation } \\
\text { (3) }\end{array}$ & $\begin{array}{c}\text { Resus- } \\
\text { pension } \\
(4)\end{array}$ & $\begin{array}{c}\text { Plume } \\
\text { Passage } \\
(1)+(3)\end{array}$ \\
\hline Au-199 & 8.74E-06 & $1.88 \mathrm{E}-07$ & $8.55 \mathrm{E}-06$ & $0.00 \mathrm{E}+00$ & $0.00 \mathrm{E}+00$ & 1.88E-07 \\
\hline $\mathrm{Hg}-194$ & 5.94E-04 & $5.30 \mathrm{E}-08$ & 1.19E-04 & $4.53 \mathrm{E}-04$ & $2.16 \mathrm{E}-05$ & $4.53 \mathrm{E}-04$ \\
\hline $\mathrm{Hg}-197$ & 1.39E-05 & $1.30 \mathrm{E}-07$ & $6.09 \mathrm{E}-06$ & 7.44E-06 & $2.15 \mathrm{E}-07$ & 7.57E-06 \\
\hline $\mathrm{Hg}-197 \mathrm{~m}$ & 1.85E-05 & 1.86E-07 & 5.39E-06 & $1.26 \mathrm{E}-05$ & 2.62E-07 & $1.28 \mathrm{E}-05$ \\
\hline $\mathrm{Hg}-199 \mathrm{~m}$ & 1.86E-07 & $0.00 \mathrm{E}+00$ & $0.00 \mathrm{E}+00$ & 1.86E-07 & $1.35 \mathrm{E}-10$ & 1.86E-07 \\
\hline $\mathrm{Hg}-203$ & 1.13E-04 & $4.81 \mathrm{E}-07$ & $3.96 \mathrm{E}-05$ & $6.94 \mathrm{E}-05$ & $3.16 \mathrm{E}-06$ & $6.99 \mathrm{E}-05$ \\
\hline TI-200 & 4.97E-05 & $2.74 \mathrm{E}-06$ & 4.70E-05 & $0.00 \mathrm{E}+00$ & $0.00 \mathrm{E}+00$ & $2.74 \mathrm{E}-06$ \\
\hline TI-201 & 8.99E-06 & $1.80 \mathrm{E}-07$ & $8.81 \mathrm{E}-06$ & $0.00 \mathrm{E}+00$ & $0.00 \mathrm{E}+00$ & $1.80 \mathrm{E}-07$ \\
\hline TI-202 & $8.10 \mathrm{E}-05$ & $9.69 \mathrm{E}-07$ & $6.83 \mathrm{E}-05$ & 1.13E-05 & 4.70E-07 & $1.23 \mathrm{E}-05$ \\
\hline TI-204 & $3.23 \mathrm{E}-05$ & $2.13 \mathrm{E}-09$ & $2.14 \mathrm{E}-07$ & 3.06E-05 & 1.44E-06 & 3.06E-05 \\
\hline TI-206 & $3.12 \mathrm{E}-13$ & $0.00 \mathrm{E}+00$ & $0.00 \mathrm{E}+00$ & $3.12 \mathrm{E}-13$ & $2.27 \mathrm{E}-17$ & $3.12 \mathrm{E}-13$ \\
\hline TI-207 & $8.84 \mathrm{E}-14$ & $6.93 \mathrm{E}-14$ & $1.92 \mathrm{E}-14$ & $0.00 \mathrm{E}+00$ & $0.00 \mathrm{E}+00$ & $6.93 \mathrm{E}-14$ \\
\hline TI-208 & $3.05 \mathrm{E}-13$ & $2.53 \mathrm{E}-13$ & $5.24 \mathrm{E}-14$ & $0.00 \mathrm{E}+00$ & $0.00 \mathrm{E}+00$ & $2.53 \mathrm{E}-13$ \\
\hline TI-209 & 9.79E-09 & $1.50 \mathrm{E}-16$ & $3.15 \mathrm{E}-17$ & $9.76 \mathrm{E}-09$ & $3.10 \mathrm{E}-11$ & 9.76E-09 \\
\hline $\mathrm{Pb}-202$ & 1.39E-03 & 2.93E-09 & 1.46E-05 & $1.31 \mathrm{E}-03$ & $6.20 \mathrm{E}-05$ & $1.31 \mathrm{E}-03$ \\
\hline $\mathrm{Pb}-203$ & 2.72E-05 & $6.19 \mathrm{E}-07$ & $2.21 \mathrm{E}-05$ & 4.34E-06 & 1.14E-07 & 4.96E-06 \\
\hline Pb-205 & 3.39E-05 & $1.21 \mathrm{E}-10$ & $1.26 \mathrm{E}-07$ & $3.22 \mathrm{E}-05$ & $1.52 \mathrm{E}-06$ & $3.22 \mathrm{E}-05$ \\
\hline Pb-209 & 8.59E-07 & $0.00 \mathrm{E}+00$ & $0.00 \mathrm{E}+00$ & 8.56E-07 & 2.72E-09 & 8.56E-07 \\
\hline $\mathrm{Pb}-210$ & 1.69E-01 & $2.78 \mathrm{E}-09$ & 4.94E-07 & 1.62E-01 & 7.65E-03 & 1.62E-01 \\
\hline $\mathrm{Pb}-211$ & 8.50E-08 & $5.09 \mathrm{E}-08$ & $3.41 \mathrm{E}-08$ & $0.00 \mathrm{E}+00$ & $0.00 \mathrm{E}+00$ & $5.09 \mathrm{E}-08$ \\
\hline $\mathrm{Pb}-212$ & 2.37E-05 & $2.12 \mathrm{E}-06$ & $2.16 \mathrm{E}-05$ & $0.00 \mathrm{E}+00$ & $0.00 \mathrm{E}+00$ & $2.12 \mathrm{E}-06$ \\
\hline $\mathrm{Pb}-214$ & 1.77E-06 & $9.79 \mathrm{E}-07$ & $5.30 \mathrm{E}-07$ & $2.45 \mathrm{E}-07$ & $1.74 \mathrm{E}-08$ & $1.22 \mathrm{E}-06$ \\
\hline Bi-206 & 3.81E-04 & $7.18 \mathrm{E}-06$ & $3.74 \mathrm{E}-04$ & $0.00 \mathrm{E}+00$ & $0.00 \mathrm{E}+00$ & $7.18 \mathrm{E}-06$ \\
\hline $\mathrm{Bi}-207$ & 5.01E-04 & $3.35 \mathrm{E}-06$ & $2.53 \mathrm{E}-04$ & $2.34 \mathrm{E}-04$ & 1.10E-05 & 2.37E-04 \\
\hline $\mathrm{Bi}-210$ & 2.42E-03 & $4.96 \mathrm{E}-15$ & $1.86 \mathrm{E}-11$ & $2.30 \mathrm{E}-03$ & 1.24E-04 & $2.30 \mathrm{E}-03$ \\
\hline $\mathrm{Bi}-210 \mathrm{~m}$ & 9.38E-02 & $0.00 \mathrm{E}+00$ & $0.00 \mathrm{E}+00$ & 8.96E-02 & $4.22 \mathrm{E}-03$ & 8.96E-02 \\
\hline Bi-211 & $7.18 \mathrm{E}-14$ & $5.62 \mathrm{E}-14$ & $1.56 \mathrm{E}-14$ & $0.00 \mathrm{E}+00$ & $0.00 \mathrm{E}+00$ & $5.62 \mathrm{E}-14$ \\
\hline $\mathrm{Bi}-212$ & $2.55 \mathrm{E}-06$ & $1.48 \mathrm{E}-06$ & 1.07E-06 & $0.00 \mathrm{E}+00$ & $0.00 \mathrm{E}+00$ & $1.48 \mathrm{E}-06$ \\
\hline $\mathrm{Bi}-213$ & 3.74E-07 & $1.25 \mathrm{E}-07$ & $9.44 \mathrm{E}-08$ & $1.54 \mathrm{E}-07$ & $7.46 \mathrm{E}-10$ & $2.79 \mathrm{E}-07$ \\
\hline $\mathrm{Bi}-214$ & 5.99E-07 & $2.36 \mathrm{E}-07$ & $9.48 \mathrm{E}-08$ & $2.55 \mathrm{E}-07$ & $1.30 \mathrm{E}-08$ & 4.91E-07 \\
\hline Po-210 & 1.06E-01 & $1.87 \mathrm{E}-11$ & 1.41E-09 & 1.02E-01 & $4.74 \mathrm{E}-03$ & $1.02 \mathrm{E}-01$ \\
\hline Po-211 & $0.00 \mathrm{E}+00$ & $0.00 \mathrm{E}+00$ & $0.00 \mathrm{E}+00$ & $0.00 \mathrm{E}+00$ & $0.00 \mathrm{E}+00$ & $0.00 \mathrm{E}+00$ \\
\hline Po-213 & $3.08 \mathrm{E}-16$ & $0.00 \mathrm{E}+00$ & $0.00 \mathrm{E}+00$ & $3.07 \mathrm{E}-16$ & 7.96E-19 & 3.07E-16 \\
\hline Po-214 & $3.95 \mathrm{E}-14$ & $6.48 \mathrm{E}-22$ & 1.15E-19 & 3.77E-14 & $1.78 \mathrm{E}-15$ & 3.77E-14 \\
\hline Po-215 & $6.98 \mathrm{E}-14$ & $4.18 \mathrm{E}-14$ & $2.80 \mathrm{E}-14$ & $0.00 \mathrm{E}+00$ & $0.00 \mathrm{E}+00$ & $4.18 \mathrm{E}-14$ \\
\hline Po-216 & $9.29 \mathrm{E}-11$ & $8.30 \mathrm{E}-12$ & $8.46 \mathrm{E}-11$ & $0.00 \mathrm{E}+00$ & $0.00 \mathrm{E}+00$ & $8.30 \mathrm{E}-12$ \\
\hline Po-218 & $2.15 \mathrm{E}-07$ & $1.20 \mathrm{E}-07$ & $6.59 \mathrm{E}-08$ & $2.66 \mathrm{E}-08$ & 1.98E-09 & 1.47E-07 \\
\hline At-211 & $3.25 \mathrm{E}-06$ & $3.64 \mathrm{E}-08$ & $3.22 \mathrm{E}-06$ & $2.32 \mathrm{E}-10$ & $5.22 \mathrm{E}-10$ & $3.66 \mathrm{E}-08$ \\
\hline At-217 & $4.41 \mathrm{E}-12$ & $1.47 \mathrm{E}-12$ & $1.11 \mathrm{E}-12$ & $1.82 \mathrm{E}-12$ & $8.80 \mathrm{E}-15$ & $3.29 \mathrm{E}-12$ \\
\hline Rn-218 & $8.41 \mathrm{E}-12$ & $1.38 \mathrm{E}-19$ & $2.46 \mathrm{E}-17$ & 8.03E-12 & $3.80 \mathrm{E}-13$ & 8.03E-12 \\
\hline $\mathrm{Rn}-219$ & $1.56 \mathrm{E}-10$ & $9.32 \mathrm{E}-11$ & $6.25 \mathrm{E}-11$ & $0.00 \mathrm{E}+00$ & $0.00 \mathrm{E}+00$ & $9.32 \mathrm{E}-11$ \\
\hline
\end{tabular}


Table 4. Ground release, Class F, $1 \mathrm{~m} / \mathrm{s}$ wind, $1 \mathrm{~km}$, no rain, continued.

\begin{tabular}{|c|c|c|c|c|c|c|}
\hline Nuclide & $\begin{array}{c}\text { 7-day } \\
\text { Total } \\
(1+2+3+4)\end{array}$ & $\begin{array}{c}\text { Cloud } \\
\text { shine } \\
(1)\end{array}$ & $\begin{array}{c}\text { Ground } \\
\text { shine } \\
(2)\end{array}$ & $\begin{array}{c}\text { Inhalation } \\
\text { (3) }\end{array}$ & $\begin{array}{c}\text { Resus- } \\
\text { pension } \\
(4)\end{array}$ & $\begin{array}{c}\text { Plume } \\
\text { Passage } \\
(1)+(3)\end{array}$ \\
\hline Rn-220 & $3.44 \mathrm{E}-08$ & 3.05E-09 & $3.14 \mathrm{E}-08$ & $0.00 \mathrm{E}+00$ & $0.00 \mathrm{E}+00$ & 3.05E-09 \\
\hline Rn-222 & 4.84E-06 & $2.59 \mathrm{E}-06$ & $2.01 \mathrm{E}-06$ & $2.04 \mathrm{E}-07$ & 3.41E-08 & $2.79 \mathrm{E}-06$ \\
\hline Fr-221 & $4.14 \mathrm{E}-08$ & 1.46E-08 & $1.11 \mathrm{E}-08$ & 1.56E-08 & $7.96 \mathrm{E}-11$ & $3.02 \mathrm{E}-08$ \\
\hline Fr-223 & 1.22E-04 & 9.03E-09 & $6.25 \mathrm{E}-08$ & 1.17E-04 & $5.28 \mathrm{E}-06$ & 1.17E-04 \\
\hline Ra-222 & 9.13E-09 & $1.50 \mathrm{E}-16$ & $2.67 \mathrm{E}-14$ & 8.72E-09 & $4.13 \mathrm{E}-10$ & 8.72E-09 \\
\hline Ra-223 & 1.00E-01 & $5.13 \mathrm{E}-07$ & 4.32E-05 & $9.65 \mathrm{E}-02$ & 3.99E-03 & 9.65E-02 \\
\hline Ra-224 & 4.33E-02 & $1.28 \mathrm{E}-07$ & $1.26 \mathrm{E}-04$ & $4.18 \mathrm{E}-02$ & 1.35E-03 & 4.18E-02 \\
\hline Ra-225 & 9.52E-02 & $1.49 \mathrm{E}-08$ & $9.50 \mathrm{E}-06$ & $9.08 \mathrm{E}-02$ & 4.46E-03 & $9.08 \mathrm{E}-02$ \\
\hline Ra-226 & 8.63E-01 & $2.62 \mathrm{E}-08$ & $1.20 \mathrm{E}-04$ & $8.24 \mathrm{E}-01$ & $3.88 \mathrm{E}-02$ & $8.24 \mathrm{E}-01$ \\
\hline Ra-228 & $2.09 \mathrm{E}-01$ & 2.73E-07 & 1.44E-04 & 2.00E-01 & $9.81 \mathrm{E}-03$ & $2.00 \mathrm{E}-01$ \\
\hline Ac-225 & 1.05E-01 & $3.48 \mathrm{E}-07$ & $3.15 \mathrm{E}-05$ & $1.01 \mathrm{E}-01$ & $4.08 \mathrm{E}-03$ & 1.01E-01 \\
\hline Ac-227 & $1.58 \mathrm{E}+01$ & 1.92E-09 & $3.31 \mathrm{E}-06$ & $1.51 \mathrm{E}+01$ & $7.10 \mathrm{E}-01$ & $1.51 \mathrm{E}+01$ \\
\hline Ac-228 & 1.54E-03 & 1.76E-06 & 7.33E-06 & 1.46E-03 & $6.86 \mathrm{E}-05$ & 1.46E-03 \\
\hline Th-226 & 3.79E-07 & 6.86E-09 & 4.42E-09 & $3.48 \mathrm{E}-07$ & 2.01E-08 & $3.55 \mathrm{E}-07$ \\
\hline Th-227 & 1.98E-01 & $2.21 \mathrm{E}-07$ & $2.64 \mathrm{E}-05$ & 1.89E-01 & $\mathrm{E}-03$ & 1.89E-01 \\
\hline Th-228 & $4.16 \mathrm{E}+00$ & 4.49E-09 & 1.19E-05 & $3.97 \mathrm{E}+00$ & 1.87E-01 & $3.97 \mathrm{E}+00$ \\
\hline Th-229 & $2.11 \mathrm{E}+01$ & $1.81 \mathrm{E}-07$ & $1.85 \mathrm{E}-05$ & $2.02 \mathrm{E}+01$ & E-01 & $2.02 \mathrm{E}+01$ \\
\hline Th-230 & $3.19 \mathrm{E}+00$ & $8.20 \mathrm{E}-10$ & $1.51 \mathrm{E}-07$ & $3.05 E+00$ & $1.43 \mathrm{E}-01$ & $3.05 \mathrm{E}+00$ \\
\hline Th-231 & $1.13 \mathrm{E}-05$ & $2.35 \mathrm{E}-08$ & $6.77 \mathrm{E}-07$ & $1.04 \mathrm{E}-05$ & 2.09E-07 & 1.04E-05 \\
\hline Th-232 & $1.41 \mathrm{E}+01$ & $3.93 \mathrm{E}-10$ & $2.70 \mathrm{E}-07$ & $1.34 \mathrm{E}+01$ & $6.33 \mathrm{E}-01$ & $1.34 \mathrm{E}+01$ \\
\hline Th-234 & 4.30E-04 & $4.29 \mathrm{E}-08$ & $4.14 \mathrm{E}-06$ & $4.08 \mathrm{E}-04$ & 1.80E-05 & $4.08 \mathrm{E}-04$ \\
\hline Pa-230 & 9.87E-05 & 1.43E-06 & $9.72 \mathrm{E}-05$ & 3.61E-09 & 6.87E-09 & 1.43E-06 \\
\hline Pa-231 & $1.05 \mathrm{E}+01$ & $6.34 \mathrm{E}-08$ & $5.98 \mathrm{E}-06$ & $9.99 \mathrm{E}+00$ & $4.71 \mathrm{E}-01$ & $9.99 \mathrm{E}+00$ \\
\hline Pa-232 & $8.54 \mathrm{E}-04$ & $7.72 \mathrm{E}-16$ & $6.47 \mathrm{E}-12$ & $8.27 \mathrm{E}-04$ & $2.70 \mathrm{E}-05$ & $8.27 \mathrm{E}-04$ \\
\hline Pa-233 & 1.48E-04 & 4.47E-07 & $3.60 \mathrm{E}-05$ & 1.07E-04 & 4.77E-06 & 1.07E-04 \\
\hline Pa-234 & 2.11E-05 & $3.74 \mathrm{E}-06$ & $1.74 \mathrm{E}-05$ & $5.96 \mathrm{E}-10$ & $2.01 \mathrm{E}-10$ & $3.74 \mathrm{E}-06$ \\
\hline $\mathrm{Pa}-234 \mathrm{~m}$ & $9.47 \mathrm{E}-11$ & $1.42 \mathrm{E}-11$ & $6.59 \mathrm{E}-11$ & $1.40 \mathrm{E}-11$ & $6.60 \mathrm{E}-13$ & $2.82 \mathrm{E}-11$ \\
\hline U-230 & 5.07E-06 & 3.33E-08 & $3.27 \mathrm{E}-06$ & $3.75 \mathrm{E}-07$ & $1.39 \mathrm{E}-06$ & $4.08 \mathrm{E}-07$ \\
\hline U-231 & 8.80E-06 & 1.43E-07 & $8.53 \mathrm{E}-06$ & $5.11 \mathrm{E}-08$ & $7.90 \mathrm{E}-08$ & 1.94E-07 \\
\hline U-232 & $8.12 \mathrm{E}+00$ & $5.58 \mathrm{E}-10$ & $2.01 \mathrm{E}-07$ & $7.76 \mathrm{E}+00$ & 3.66E-01 & $7.76 \mathrm{E}+00$ \\
\hline U-233 & $1.64 \mathrm{E}+00$ & $5.03 \mathrm{E}-10$ & $8.34 \mathrm{E}-08$ & $1.57 \mathrm{E}+00$ & 7.39E-02 & $1.57 \mathrm{E}+00$ \\
\hline U-234 & $1.61 \mathrm{E}+00$ & $3.21 \mathrm{E}-10$ & $1.35 \mathrm{E}-07$ & $1.54 \mathrm{E}+00$ & 7.26E-02 & $1.54 \mathrm{E}+00$ \\
\hline U-235 & $1.48 \mathrm{E}+00$ & $3.24 \mathrm{E}-07$ & $3.10 \mathrm{E}-05$ & $1.41 \mathrm{E}+00$ & $6.65 \mathrm{E}-02$ & $1.41 \mathrm{E}+00$ \\
\hline U-236 & $1.52 \mathrm{E}+00$ & $2.53 \mathrm{E}-10$ & $1.23 \mathrm{E}-07$ & $1.45 \mathrm{E}+00$ & $6.85 \mathrm{E}-02$ & $1.45 \mathrm{E}+00$ \\
\hline U-237 & 1.93E-05 & $2.80 \mathrm{E}-07$ & $1.90 \mathrm{E}-05$ & $2.75 \mathrm{E}-10$ & $4.71 \mathrm{E}-10$ & $2.80 \mathrm{E}-07$ \\
\hline U-238 & $1.43 \mathrm{E}+00$ & $2.81 \mathrm{E}-10$ & 5.37E-07 & $1.37 \mathrm{E}+00$ & $6.46 \mathrm{E}-02$ & $1.37 \mathrm{E}+00$ \\
\hline U-239 & 3.31E-07 & $1.24 \mathrm{E}-08$ & $9.99 \mathrm{E}-08$ & 2.14E-07 & 4.78E-09 & 2.26E-07 \\
\hline U-240 & 4.79E-05 & $6.73 \mathrm{E}-07$ & $6.63 \mathrm{E}-06$ & 4.01E-05 & 4.94E-07 & $4.08 \mathrm{E}-05$ \\
\hline $\mathrm{Np}-235$ & 7.91E-07 & 4.21E-09 & 7.87E-07 & $6.34 \mathrm{E}-13$ & $1.29 \mathrm{E}-12$ & 4.21E-09 \\
\hline Np-236a & $1.23 \mathrm{E}+00$ & $2.75 \mathrm{E}-07$ & 2.63E-05 & $1.18 \mathrm{E}+00$ & $5.55 \mathrm{E}-02$ & $1.18 \mathrm{E}+00$ \\
\hline Np-236b & 5.09E-05 & $1.04 \mathrm{E}-07$ & $1.86 \mathrm{E}-06$ & $2.69 \mathrm{E}-05$ & $2.20 \mathrm{E}-05$ & 2.70E-05 \\
\hline
\end{tabular}


Table 4. Ground release, Class F, $1 \mathrm{~m} / \mathrm{s}$ wind, $1 \mathrm{~km}$, no rain, continued.

\begin{tabular}{|c|c|c|c|c|c|c|}
\hline Nuclide & $\begin{array}{c}\text { 7-day } \\
\text { Total } \\
(1+2+3+4)\end{array}$ & $\begin{array}{c}\text { Cloud } \\
\text { shine } \\
(1)\end{array}$ & $\begin{array}{c}\text { Ground } \\
\text { shine } \\
(2)\end{array}$ & $\begin{array}{c}\text { Inhalation } \\
\text { (3) }\end{array}$ & $\begin{array}{c}\text { Resus- } \\
\text { pension } \\
(4)\end{array}$ & $\begin{array}{c}\text { Plume } \\
\text { Passage } \\
(1)+(3)\end{array}$ \\
\hline $\mathrm{Np}-237$ & $6.11 \mathrm{E}+00$ & $4.88 \mathrm{E}-08$ & 8.73E-06 & $5.83 \mathrm{E}+00$ & $2.75 \mathrm{E}-01$ & $5.83 \mathrm{E}+00$ \\
\hline $\mathrm{Np}-238$ & 4.54E-05 & $1.20 \mathrm{E}-06$ & 3.49E-05 & $4.16 \mathrm{E}-06$ & 5.19E-06 & 5.36E-06 \\
\hline Np-239 & 3.86E-05 & $3.47 \mathrm{E}-07$ & $1.34 \mathrm{E}-05$ & $2.42 \mathrm{E}-05$ & $6.81 \mathrm{E}-07$ & $2.45 \mathrm{E}-05$ \\
\hline $\mathrm{Np}-240$ & $2.21 \mathrm{E}-06$ & $1.10 \mathrm{E}-06$ & 1.07E-06 & 4.16E-08 & 3.47E-09 & 1.14E-06 \\
\hline Np-240m & 9.57E-09 & $5.40 \mathrm{E}-10$ & $1.71 \mathrm{E}-10$ & 8.46E-09 & $3.99 \mathrm{E}-10$ & $9.00 \mathrm{E}-09$ \\
\hline Pu-236 & $1.69 \mathrm{E}+00$ & $2.51 \mathrm{E}-10$ & $1.66 \mathrm{E}-07$ & $1.61 \mathrm{E}+00$ & 7.59E-02 & $1.61 \mathrm{E}+00$ \\
\hline Pu-237 & 9.46E-06 & $1.00 \mathrm{E}-07$ & $9.36 \mathrm{E}-06$ & $2.75 \mathrm{E}-10$ & $5.47 \mathrm{E}-10$ & 1.00E-07 \\
\hline Pu-238 & $3.82 \mathrm{E}+00$ & $1.86 \mathrm{E}-10$ & 1.44E-07 & $3.65 \mathrm{E}+00$ & $1.72 \mathrm{E}-01$ & $3.65 \mathrm{E}+00$ \\
\hline Pu-239 & $4.12 \mathrm{E}+00$ & $1.72 \mathrm{E}-10$ & $6.33 \mathrm{E}-08$ & $3.94 \mathrm{E}+00$ & 1.86E-01 & $3.94 \mathrm{E}+00$ \\
\hline Pu-240 & $4.13 \mathrm{E}+00$ & $1.82 \mathrm{E}-10$ & $1.37 \mathrm{E}-07$ & $3.94 \mathrm{E}+00$ & 1.86E-01 & $3.94 \mathrm{E}+00$ \\
\hline Pu-241 & $6.98 \mathrm{E}-02$ & $4.69 \mathrm{E}-14$ & $2.66 \mathrm{E}-10$ & $6.66 \mathrm{E}-02$ & $3.14 \mathrm{E}-03$ & $6.66 \mathrm{E}-02$ \\
\hline Pu-242 & $3.92 \mathrm{E}+00$ & $1.55 \mathrm{E}-10$ & 1.14E-07 & $3.75 \mathrm{E}+00$ & 1.76E-01 & $3.75 \mathrm{E}+00$ \\
\hline Pu-243 & $3.20 \mathrm{E}-07$ & 4.06E-08 & $1.82 \mathrm{E}-07$ & 7.76E-08 & $2.05 \mathrm{E}-08$ & 1.18E-07 \\
\hline Pu-244 & $3.71 \mathrm{E}+00$ & $3.75 \mathrm{E}-08$ & 4.97E-05 & $3.54 \mathrm{E}+00$ & 1.67E-01 & $3.54 \mathrm{E}+00$ \\
\hline Pu-245 & 7.66E-06 & $8.49 \mathrm{E}-07$ & $6.76 \mathrm{E}-06$ & 1.39E-08 & 3.34E-08 & 8.63E-07 \\
\hline Pu-246 & 1.44E-04 & 1.79E-06 & $1.42 \mathrm{E}-04$ & 6.09E-08 & 2.41E-07 & 1.85E-06 \\
\hline Am-241 & $6.50 \mathrm{E}+00$ & $3.95 \mathrm{E}-08$ & $4.92 \mathrm{E}-06$ & $6.21 \mathrm{E}+00$ & $2.92 \mathrm{E}-01$ & $6.21 \mathrm{E}+00$ \\
\hline Am-242 & 7.37E-04 & $2.74 \mathrm{E}-08$ & 4.04E-07 & 7.04E-04 & $3.30 \mathrm{E}-05$ & 7.04E-04 \\
\hline$A m-242 m$ & $6.27 \mathrm{E}+00$ & $2.65 \mathrm{E}-09$ & $3.34 \mathrm{E}-06$ & $5.99 \mathrm{E}+00$ & $2.82 \mathrm{E}-01$ & $5.99 \mathrm{E}+00$ \\
\hline Am-243 & $6.48 \mathrm{E}+00$ & 1.12E-07 & $2.95 \mathrm{E}-05$ & $6.19 \mathrm{E}+00$ & 2.92E-01 & $6.19 \mathrm{E}+00$ \\
\hline Am-244 & 3.87E-05 & $1.60 \mathrm{E}-06$ & $1.13 \mathrm{E}-05$ & 1.76E-05 & $8.20 \mathrm{E}-06$ & $1.92 \mathrm{E}-05$ \\
\hline Am-245 & 1.92E-07 & $4.28 \mathrm{E}-08$ & 7.96E-08 & $6.17 \mathrm{E}-08$ & 8.05E-09 & 1.05E-07 \\
\hline $\mathrm{Am}-246$ & 9.75E-07 & $5.49 \mathrm{E}-07$ & $3.47 \mathrm{E}-07$ & 7.33E-08 & 4.63E-09 & $6.22 \mathrm{E}-07$ \\
\hline Cm-242 & 2.17E-01 & $2.07 \mathrm{E}-10$ & $1.54 \mathrm{E}-07$ & $2.08 \mathrm{E}-01$ & $9.70 \mathrm{E}-03$ & $2.08 \mathrm{E}-01$ \\
\hline $\mathrm{Cm}-243$ & $4.34 \mathrm{E}+00$ & $2.68 \mathrm{E}-07$ & $2.43 \mathrm{E}-05$ & $4.15 \mathrm{E}+00$ & 1.95E-01 & $4.15 \mathrm{E}+00$ \\
\hline Cm-244 & $3.45 \mathrm{E}+00$ & 1.76E-10 & $1.39 \mathrm{E}-07$ & $3.30 \mathrm{E}+00$ & $1.55 \mathrm{E}-01$ & $3.30 \mathrm{E}+00$ \\
\hline $\mathrm{Cm}-245$ & $6.69 \mathrm{E}+00$ & $1.50 \mathrm{E}-07$ & $1.42 \mathrm{E}-05$ & $6.39 \mathrm{E}+00$ & 3.01E-01 & $6.39 \mathrm{E}+00$ \\
\hline $\mathrm{Cm}-246$ & $6.58 \mathrm{E}+00$ & $1.48 \mathrm{E}-10$ & 1.23E-07 & $6.29 \mathrm{E}+00$ & 2.96E-01 & $6.29 \mathrm{E}+00$ \\
\hline $\mathrm{Cm}-247$ & $6.15 \mathrm{E}-05$ & $6.75 \mathrm{E}-07$ & $6.07 \mathrm{E}-05$ & 7.14E-09 & $1.53 \mathrm{E}-07$ & $6.82 \mathrm{E}-07$ \\
\hline Cm-248 & $5.71 \mathrm{E}+00$ & $1.30 \mathrm{E}-10$ & $9.84 \mathrm{E}-08$ & $5.45 \mathrm{E}+00$ & $2.57 \mathrm{E}-01$ & $5.45 \mathrm{E}+00$ \\
\hline Cm-249 & 3.49E-08 & 1.76E-08 & 1.73E-08 & $7.10 \mathrm{E}-18$ & $4.94 \mathrm{E}-16$ & 1.76E-08 \\
\hline $\mathrm{Cm}-250$ & 2.82E-05 & $6.68 \mathrm{E}-08$ & 2.82E-05 & $1.90 \mathrm{E}-11$ & 7.20E-09 & $6.68 \mathrm{E}-08$ \\
\hline Bk-249 & $1.21 \mathrm{E}-09$ & $5.35 \mathrm{E}-13$ & $1.20 \mathrm{E}-09$ & $2.18 \mathrm{E}-13$ & $3.66 \mathrm{E}-12$ & $7.53 \mathrm{E}-13$ \\
\hline Bk-250 & 4.84E-06 & $1.49 \mathrm{E}-06$ & $3.35 \mathrm{E}-06$ & $4.74 \mathrm{E}-13$ & $7.08 \mathrm{E}-12$ & 1.49E-06 \\
\hline Cf-248 & 5.59E-05 & $1.42 \mathrm{E}-10$ & 1.12E-07 & $1.84 \mathrm{E}-05$ & $3.74 \mathrm{E}-05$ & $1.84 \mathrm{E}-05$ \\
\hline Cf-249 & 5.97E-05 & 6.93E-07 & $5.88 \mathrm{E}-05$ & 7.60E-08 & $1.55 \mathrm{E}-07$ & 7.69E-07 \\
\hline Cf-250 & 5.23E-07 & $1.81 \mathrm{E}-10$ & $1.15 \mathrm{E}-07$ & 1.34E-07 & $2.74 \mathrm{E}-07$ & 1.34E-07 \\
\hline Cf-251 & $2.28 \mathrm{E}-05$ & 2.47E-07 & $2.26 \mathrm{E}-05$ & $2.56 \mathrm{E}-19$ & $3.26 \mathrm{E}-17$ & 2.47E-07 \\
\hline Cf-252 & 1.11E-07 & $1.58 \mathrm{E}-10$ & 1.06E-07 & 1.57E-09 & 3.21E-09 & 1.73E-09 \\
\hline Cf-253 & $2.26 \mathrm{E}-08$ & $7.39 \mathrm{E}-11$ & $2.25 \mathrm{E}-08$ & $7.75 \mathrm{E}-21$ & $6.12 \mathrm{E}-16$ & 7.39E-11 \\
\hline Cf-254 & $3.81 \mathrm{E}-12$ & $2.67 \mathrm{E}-14$ & $3.79 \mathrm{E}-12$ & $2.74 \mathrm{E}-22$ & $8.64 \mathrm{E}-18$ & $2.67 \mathrm{E}-14$ \\
\hline
\end{tabular}


Table 4. Ground release, Class F, $1 \mathrm{~m} / \mathrm{s}$ wind, $1 \mathrm{~km}$, no rain, continued.

\begin{tabular}{|l|c|c|c|c|c|r|}
\hline Nuclide & $\begin{array}{c}\text { 7-day } \\
\text { Total } \\
(1+2+3+4)\end{array}$ & $\begin{array}{c}\text { Cloud } \\
\text { shine } \\
(1)\end{array}$ & $\begin{array}{c}\text { Ground } \\
\text { shine } \\
(2)\end{array}$ & $\begin{array}{c}\text { Inhalation } \\
(3)\end{array}$ & $\begin{array}{c}\text { Resus- } \\
\text { pension } \\
(4)\end{array}$ & $\begin{array}{c}\text { Plume } \\
\text { Passage } \\
(1)+(3)\end{array}$ \\
\hline Es-253 & $1.07 \mathrm{E}-07$ & $7.04 \mathrm{E}-10$ & $1.06 \mathrm{E}-07$ & $8.66 \mathrm{E}-18$ & $1.28 \mathrm{E}-14$ & $7.04 \mathrm{E}-10$ \\
\hline $\mathrm{Es}-254$ & $1.41 \mathrm{E}-04$ & $4.77 \mathrm{E}-07$ & $1.41 \mathrm{E}-04$ & $4.44 \mathrm{E}-14$ & $6.58 \mathrm{E}-11$ & $4.77 \mathrm{E}-07$ \\
\hline Es-254m & $3.17 \mathrm{E}-05$ & $1.18 \mathrm{E}-06$ & $3.05 \mathrm{E}-05$ & $4.32 \mathrm{E}-14$ & $3.87 \mathrm{E}-11$ & $1.18 \mathrm{E}-06$ \\
\hline Fm-254 & $3.07 \mathrm{E}-09$ & $1.79 \mathrm{E}-10$ & $2.88 \mathrm{E}-09$ & $4.74 \mathrm{E}-13$ & $7.12 \mathrm{E}-12$ & $1.79 \mathrm{E}-10$ \\
\hline Fm-255 & $2.08 \mathrm{E}-07$ & $4.24 \mathrm{E}-09$ & $2.04 \mathrm{E}-07$ & $1.06 \mathrm{E}-25$ & $2.42 \mathrm{E}-23$ & $4.24 \mathrm{E}-09$ \\
\hline & & & & & & \\
\hline
\end{tabular}




\section{SPECIFIC DOSE DATA TABLE FOR ROUTINE RELEASES}

This chapter provides some data for MEI and population average doses from routinely released radionuclides. Since these calculations would be site-dependent on many factors - weather, the proximate vegetation and animal food chains near the fusion facility, and the population living near the facility - some assumptions were made. These assumptions are outlined below. The releases are elevated stack releases, assuming a 100-m high stack, and a 2-km site boundary.

Long-term dispersion factors $(\chi / \mathrm{Q})$ were calculated for a set of 565 fusion radionuclides for 18 distances ranging from $0.1-80 \mathrm{~km}$. Doses were calculated using a modified version of the Radiological Safety Analysis Computer Program (RSAC-5f) which was expanded for fusion studies to evaluate a larger radionuclide data base. Dose impacts were evaluated for a MEI member of the public located at 2-km from the release point and for an average population $\left(100 \mathrm{~km}^{-2}\right)$ from 2 to $100 \mathrm{~km}$ from the release point. $\chi / \mathrm{Q}$ 's were determined using the U.S. Environmental Protection Agency's CAP88-PC code, which uses the1973 Briggs dispersion parameters (sigma-y and sigma-z) [23]. Five years of meteorological statistics from the INEEL since the laboratory has an excellent meteorological monitoring data base. Alternate $\chi / Q$ values are provided for ten alternative locations in the US [2].

Average wind speed is required for nuclide decay and ingrowth calculations during downwind transport. The average hourly wind speed at the INEEL Grid 3 tower $(3.6 \mathrm{~m} / \mathrm{s})$ was determined by examining hourly data from 1988 to 1991 , this value is used for average wind speed in the calculations. The average annual mixing height at the INEEL has been estimated to be $800 \mathrm{~m}$. The mixing height effectively bounds vertical expansion of the plume (sigma-z). In general, this parameter will only effect concentrations during unstable conditions ( $\mathrm{P}-\mathrm{G}$ classes A through $\mathrm{C}$ ) at distances greater than $2 \mathrm{~km}[2]$.

For the MEI calculations $\chi / Q$ data base, $\chi / Q$ 's were evaluated at eighteen downwind distances $(0.1,1-10 \mathrm{~km}$ [1 km increments], and 10-80 km [10 km increments]) in each of the sixteen $22.5^{\circ}$ sectors. The maximum sector $\chi / Q$ (generally towards the southsouthwest at the INEEL) for each receptor distance was selected. For the population dose calculations, $\chi / Q$ 's were calculated at the midpoints of six distance intervals ranging from 2 to $100 \mathrm{~km}(9.1,24.2,40.3,56.4,72.5$, and $90.3 \mathrm{~km})$. Since these calculations assume a uniform population density of $100 \mathrm{~km}^{-2}$, individual sector $\chi / \mathrm{Q}$ differences are irrelevant and the average $\chi / Q$ over all 16 sectors (at each interval midpoint) was used in the dose calculations. All $\chi / Q$ s were "sector-averaged" which uniformly disperses the plume laterally across a $22.5^{\circ}$ sector when the wind blows in that direction. This method is more appropriate for annual average calculations. The alternative "centerline" method unrealistically assumes that the plume centerline (highest concentration) is always aligned with receptor locations whenever the plume blows in a particular sector [2]. 
Four dose pathways were evaluated:

1) inhalation of radionuclides

2) ingestion of contaminated foods

3) external exposure from ground deposition of gamma-emitting radionuclides

4) external exposure from air immersion in the plume

The percentage contribution from each pathway was found for each radionuclide. For the chronic release scenario associated with routine operations, the dose from resuspension of radionuclides is small compared to direct plume inhalation and was therefore not calculated. All doses presented in this chapter are for the weighted sum of the organeffective doses and are termed effective dose (ED). This terminology is in accordance with ICRP 60 although the internal doses are calculated using ICRP 30 methodology (effective dose equivalent) [2].

Some of the yearly dose calculation assumptions are:

Dry deposition velocity for the total ground surface (accounts for gravitational settling, impaction on the ground and diffusion to the ground)

Adult breathing rate

Protection factor for inhalation

Air immersion shielding factor

Groundshine shielding factor

Human consumption rates:

milk

beef or other meat products

leafy vegetables (surface)

produce (root and grain)

produce and leafy vegetables grown in receptor's garden particulate: $\quad 0.002 \mathrm{~m} / \mathrm{s}$

halogens: $\quad 0.04 \mathrm{~m} / \mathrm{s}$

other gases: $\quad 0.0 \mathrm{~m} / \mathrm{s}$

$2.66 \mathrm{E}-04 \mathrm{~m}^{3} / \mathrm{s}$

0.75

0.75

0.33

115 1/year

$75 \mathrm{~kg} /$ year

$45 \mathrm{~kg} /$ year

$170 \mathrm{~kg} / \mathrm{year}$

1.0

Fallout interception fractions:

pasture

vegetables

0.2

iodines on forage

1.0

Weathering removal rate constant

$0.0021 /$ hour

Period of crop exposure during growing season: 
vegetables

forage

Vegetation yield:

vegetables

forage

Time of activity buildup in the soil

Soil surface density

Time delays:

fresh vegetables

stored vegetables

feed-milk-person

slaughter-consumption
60 days

30 days

$2 \mathrm{~kg} / \mathrm{m}^{2}$ (wet)

$0.28 \mathrm{~kg} / \mathrm{m}^{2}$ (dry)

15 years

$225 \mathrm{~kg} / \mathrm{m}^{2}$

1 day

60 days

2 days

20 days

The MEI calculation method provides a somewhat conservative estimate of dose because it does not account for plume depletion by dry deposition or washout at distances greater than $2 \mathrm{~km}$. Although the method accounts for radioactive decay during plume transport, it does not evaluate progeny ingrowth during transport to downwind distance greater than $2 \mathrm{~km}$ and may therefore underestimate doses for these nuclides [2].

Dose results for the 2-km MEI and the 2-100 km population are provided in Table 5, normalized to 1 Terabecquerel per nuclide per annum. The activation product particle size was assumed to be 1 micron AMAD. 
Table 5. Effective Dose for Annual Average Releases (1 TBq/a) of fusion activation products

\begin{tabular}{|c|c|c|c|c|c|c|c|}
\hline Nuclide & $\begin{array}{c}\text { Nuclide } \\
\text { Half-life } \\
\text { (days) }\end{array}$ & $\begin{array}{l}\text { MEI 2-km } \\
\text { total EDE } \\
\text { (Sv/year) }\end{array}$ & $\begin{array}{l}\text { Inhalation } \\
\% \text { of } \\
\text { total dose }\end{array}$ & $\begin{array}{l}\text { Ingestion } \\
\quad \% \text { of } \\
\text { total dose }\end{array}$ & $\begin{array}{c}\text { Ground } \\
\text { surfaces } \\
\% \text { of } \\
\text { total dose } \\
\end{array}$ & $\begin{array}{c}\text { Air } \\
\text { immersion } \\
\% \text { of } \\
\text { total dose }\end{array}$ & $\begin{array}{c}2-100 \mathrm{~km} \\
\text { population } \\
\text { dose } \\
\text { (person- } \\
\text { Sv/year) } \\
\end{array}$ \\
\hline $\mathrm{Be}-7$ & $5.34 \mathrm{E}+01$ & $2.82 \mathrm{E}-08$ & 5.6 & 13 & 80.5 & 0.9 & $1.78 \mathrm{E}-03$ \\
\hline $\mathrm{Be}-10$ & $5.84 \mathrm{E}+08$ & $2.38 \mathrm{E}-06$ & 86.6 & 13.3 & 0 & 0 & $1.51 \mathrm{E}-01$ \\
\hline C-11 & 1.42E-02 & $9.02 \mathrm{E}-11$ & 0 & 0 & 100 & 0 & $3.29 E-07$ \\
\hline C-14 & $2.09 E+06$ & $3.16 \mathrm{E}-07$ & 3.9 & 95.9 & 0 & 0 & $2.00 \mathrm{E}-02$ \\
\hline $\mathrm{N}-13$ & $6.92 \mathrm{E}-03$ & $2.70 \mathrm{E}-09$ & 0 & 0 & 0 & 100 & $2.79 \mathrm{E}-06$ \\
\hline $\mathrm{N}-16$ & $8.25 \mathrm{E}-05$ & - & - & - & - & - & $0.00 \mathrm{E}+00$ \\
\hline $0-15$ & $1.41 \mathrm{E}-03$ & $2.20 \mathrm{E}-10$ & 0 & 0 & 0 & 100 & $3.09 \mathrm{E}-11$ \\
\hline F-18 & $7.63 \mathrm{E}-02$ & $1.73 \mathrm{E}-08$ & 2.2 & 0 & 70.5 & 27.2 & $3.70 \mathrm{E}-04$ \\
\hline $\mathrm{Na}-22$ & $9.50 \mathrm{E}+02$ & $1.23 \mathrm{E}-05$ & 0.4 & 68 & 31.4 & 0.1 & $7.78 \mathrm{E}-01$ \\
\hline $\mathrm{Na}-24$ & $6.25 \mathrm{E}-01$ & $4.84 \mathrm{E}-08$ & 11.5 & 1.6 & 37.8 & 49.4 & $2.60 \mathrm{E}-03$ \\
\hline $\mathrm{Mg}-27$ & $6.57 \mathrm{E}-03$ & 2.49E-09 & 3.3 & 0 & 1 & 96 & $2.28 \mathrm{E}-06$ \\
\hline $\mathrm{Mg}-28$ & 1.56E-03 & 1.74E-09 & 77 & 0 & 0.1 & 23.1 & $6.71 \mathrm{E}-10$ \\
\hline $\mathrm{Al}-26$ & $2.61 \mathrm{E}+08$ & $6.64 \mathrm{E}-06$ & 5.2 & 15.7 & 78.9 & 0.2 & $4.21 \mathrm{E}-01$ \\
\hline Al-28 & $1.56 \mathrm{E}-03$ & $5.72 \mathrm{E}-10$ & 0.7 & 0 & 0.2 & 99.1 & $2.20 \mathrm{E}-10$ \\
\hline Si-31 & 1.09E-01 & $9.10 \mathrm{E}-10$ & 99.5 & 0 & 0.1 & 0.5 & $2.55 \mathrm{E}-05$ \\
\hline Si-32 & $1.64 \mathrm{E}+05$ & $2.55 \mathrm{E}-06$ & 10.2 & 89.8 & 0 & 0 & $1.62 \mathrm{E}-01$ \\
\hline $\mathrm{P}-32$ & $1.43 E+01$ & 3.32E-07 & 9.8 & 90.4 & 0 & 0 & $2.09 \mathrm{E}-02$ \\
\hline $\mathrm{P}-33$ & $2.54 \mathrm{E}+01$ & $7.44 \mathrm{E}-08$ & 4.8 & 95.2 & 0 & 0 & $4.70 \mathrm{E}-03$ \\
\hline S-35 & $8.74 \mathrm{E}+01$ & $1.78 \mathrm{E}-07$ & 1 & 98.9 & 0 & 0 & $1.13 \mathrm{E}-02$ \\
\hline S-37 & $3.51 \mathrm{E}-03$ & $2.86 \mathrm{E}-11$ & 100 & 0 & 0 & 0 & $3.16 \mathrm{E}-09$ \\
\hline $\mathrm{Cl}-36$ & $1.10 E+08$ & $3.67 \mathrm{E}-03$ & 0 & 100 & 0 & 0 & $2.33 E+02$ \\
\hline $\mathrm{Cl}-38$ & $2.58 \mathrm{E}-02$ & $1.26 \mathrm{E}-08$ & 4.7 & 0 & 37.9 & 57.7 & 9.54E-05 \\
\hline Cl-39 & $3.86 \mathrm{E}-02$ & $5.09 \mathrm{E}-10$ & 100 & 0 & 0 & 0 & $5.86 \mathrm{E}-06$ \\
\hline $\mathrm{Cl}-40$ & 9.38E-04 & $5.16 \mathrm{E}-13$ & 100 & 0 & 0 & 0 & $0.00 \mathrm{E}+00$ \\
\hline Ar-37 & $3.50 \mathrm{E}+01$ & $6.83 \mathrm{E}-15$ & 0 & 0 & 0 & 100 & $4.32 \mathrm{E}-10$ \\
\hline Ar-39 & $9.82 E+04$ & $0.00 \mathrm{E}+00$ & - & - & - & - & $0.00 \mathrm{E}+00$ \\
\hline Ar-41 & 7.61E-02 & $6.29 \mathrm{E}-09$ & 0 & 0 & 0 & 100 & 1.35E-04 \\
\hline K-40 & $4.66 \mathrm{E}+11$ & $1.20 \mathrm{E}-05$ & 0.6 & 96.7 & 2.4 & 0 & 7.61E-01 \\
\hline $\mathrm{K}-42$ & $5.15 \mathrm{E}-01$ & 9.08E-09 & 70.8 & 2.1 & 11.2 & 15.9 & $4.72 \mathrm{E}-04$ \\
\hline K-43 & $9.42 \mathrm{E}-01$ & 1.66E-08 & 19.8 & 3.1 & 47.3 & 29.7 & 9.42E-04 \\
\hline Ca-41 & $5.11 \mathrm{E}+07$ & 6.84E-07 & 1.1 & 98.8 & 0 & 0 & $4.34 \mathrm{E}-02$ \\
\hline Ca-45 & $1.63 E+02$ & $4.35 \mathrm{E}-07$ & 8.3 & 91.7 & 0 & 0 & $2.75 \mathrm{E}-02$ \\
\hline Ca-47 & $4.54 \mathrm{E}+00$ & $1.22 \mathrm{E}-07$ & 26.6 & 35.1 & 33.4 & 4.5 & 7.57E-03 \\
\hline Ca-49 & 6.05E-03 & 9.43E-09 & 0.4 & 0 & 0.7 & 98.9 & $7.98 \mathrm{E}-06$ \\
\hline Sc-44 & 1.64E-01 & 1.59E-08 & 15.2 & 0 & 17.2 & 67.3 & 5.64E-04 \\
\hline Sc- $44 m$ & $2.44 \mathrm{E}+00$ & 9.99E-08 & 41.8 & 16.3 & 41.4 & 0.3 & $6.55 \mathrm{E}-03$ \\
\hline Sc-46 & $8.38 \mathrm{E}+01$ & 1.86E-06 & 6.3 & 24.4 & 68.8 & 0.6 & 1.18E-01 \\
\hline Sc-47 & $3.42 \mathrm{E}+00$ & 1.87E-08 & 53.5 & 24.5 & 19 & 3 & $1.15 \mathrm{E}-03$ \\
\hline Sc-48 & $1.83 E+00$ & 9.36E-08 & 22.6 & 7.9 & 50.7 & 18.7 & $5.60 \mathrm{E}-03$ \\
\hline Sc-49 & 3.99E-02 & $4.95 \mathrm{E}-10$ & 99 & 0 & 0.1 & 1 & $5.88 \mathrm{E}-06$ \\
\hline Sc-50 & 1.19E-03 & 1.79E-12 & 100 & 0 & 0 & 0 & $2.95 \mathrm{E}-14$ \\
\hline Ti-44 & $1.73 E+04$ & 1.23E-05 & 8.1 & 53.6 & 38 & 0 & 7.78E-01 \\
\hline Ti-45 & $1.28 \mathrm{E}-01$ & 6.02E-09 & 14.2 & 0 & 15.3 & 70.4 & 1.87E-04 \\
\hline Ti-51 & $4.00 \mathrm{E}-03$ & $6.58 \mathrm{E}-10$ & 5.8 & 0 & 0.6 & 93.6 & $1.27 \mathrm{E}-07$ \\
\hline
\end{tabular}


Table 5. Effective Dose for Annual Average Releases (1 TBq/a) of fusion activation products (continued)

\begin{tabular}{|c|c|c|c|c|c|c|c|}
\hline Nuclide & $\begin{array}{c}\text { Nuclide } \\
\text { Half-life } \\
\text { (days) }\end{array}$ & $\begin{array}{l}\text { MEI 2-km } \\
\text { total EDE } \\
\text { (Sv/year) }\end{array}$ & $\begin{array}{l}\text { Inhalation } \\
\% \text { of } \\
\text { total dose }\end{array}$ & $\begin{array}{l}\text { Ingestion } \\
\% \text { of } \\
\text { total dose }\end{array}$ & $\begin{array}{c}\text { Ground } \\
\text { surfaces } \\
\% \text { of } \\
\text { total dose }\end{array}$ & $\begin{array}{c}\text { Air } \\
\text { immersion } \\
\% \text { of } \\
\text { total dose }\end{array}$ & $\begin{array}{c}2-100 \mathrm{~km} \\
\text { population } \\
\text { dose } \\
\text { (person- } \\
\text { Sv/year) } \\
\end{array}$ \\
\hline V-48 & $1.62 \mathrm{E}+01$ & $5.15 \mathrm{E}-07$ & 9.1 & 15.5 & 72.2 & 3 & $3.24 \mathrm{E}-02$ \\
\hline V-49 & $3.30 \mathrm{E}+02$ & $5.67 \mathrm{E}-09$ & 29.1 & 69 & 2 & 0 & $3.59 \mathrm{E}-04$ \\
\hline V-52 & $2.60 \mathrm{E}-03$ & 1.38E-09 & 1.3 & 0 & 0.4 & 98.6 & $3.20 \mathrm{E}-08$ \\
\hline V-53 & $1.12 \mathrm{E}-03$ & $7.72 \mathrm{E}-13$ & 100 & 0 & 0 & 0 & $5.56 \mathrm{E}-15$ \\
\hline Cr-49 & $2.92 \mathrm{E}-02$ & 5.04E-09 & 5.3 & 0 & 4.5 & 90.1 & $4.35 \mathrm{E}-05$ \\
\hline Cr-51 & $2.77 \mathrm{E}+01$ & $1.27 \mathrm{E}-08$ & 12 & 25 & 61.6 & 1.3 & $8.03 \mathrm{E}-04$ \\
\hline Mn-52 & $5.59 \mathrm{E}+00$ & $2.29 \mathrm{E}-07$ & 14.4 & 11.7 & 66.4 & 7.9 & $1.43 \mathrm{E}-02$ \\
\hline$M n-52 m$ & $1.47 \mathrm{E}-02$ & 9.59E-09 & 2.2 & 0 & 2.2 & 95.5 & $3.73 \mathrm{E}-05$ \\
\hline Mn-53 & $1.35 \mathrm{E}+09$ & $1.22 \mathrm{E}-08$ & 20.7 & 76.1 & 2.7 & 0 & 7.71E-04 \\
\hline Mn-54 & $3.13 \mathrm{E}+02$ & $1.43 \mathrm{E}-06$ & 2.6 & 12.4 & 84.6 & 0.3 & $9.05 \mathrm{E}-02$ \\
\hline Mn-56 & $1.07 \mathrm{E}-01$ & $1.17 \mathrm{E}-08$ & 11.6 & 0 & 11.7 & 76.8 & $3.24 \mathrm{E}-04$ \\
\hline Mn-57 & $1.01 \mathrm{E}-03$ & $4.90 \mathrm{E}-12$ & $9.10 \mathrm{E}+00$ & 0 & 0.2 & 90.8 & $0.00 \mathrm{E}+00$ \\
\hline Fe-52 & $3.45 \mathrm{E}-01$ & $2.77 \mathrm{E}-08$ & $4.22 \mathrm{E}+01$ & 0.9 & 31.8 & 25.1 & $1.75 \mathrm{E}-03$ \\
\hline Fe-55 & $9.86 \mathrm{E}+02$ & $1.12 \mathrm{E}-07$ & $6.30 \mathrm{E}+00$ & 93.8 & 0.4 & 0 & $7.13 \mathrm{E}-03$ \\
\hline Fe-59 & $4.46 \mathrm{E}+01$ & $8.42 \mathrm{E}-07$ & $6.90 \mathrm{E}+00$ & 44.5 & 47.9 & 0.7 & $5.33 \mathrm{E}-02$ \\
\hline Fe-60 & $3.65 \mathrm{E}+07$ & $3.10 \mathrm{E}-05$ & $5.10 \mathrm{E}+00$ & 93.9 & 1 & 0 & $1.96 \mathrm{E}+00$ \\
\hline Co-56 & $7.88 \mathrm{E}+01$ & $3.21 \mathrm{E}-06$ & $4.80 \mathrm{E}+00$ & 30.2 & 64.5 & 0.6 & $2.03 \mathrm{E}-01$ \\
\hline Co-57 & $2.71 \mathrm{E}+02$ & $3.49 \mathrm{E}-07$ & $1.27 \mathrm{E}+01$ & 31.5 & 55.6 & 0.2 & $2.21 \mathrm{E}-02$ \\
\hline Co-58 & $7.08 \mathrm{E}+01$ & $8.68 \mathrm{E}-07$ & $4.80 \mathrm{E}+00$ & 29.8 & 64.7 & 0.6 & $5.49 \mathrm{E}-02$ \\
\hline Co-58m & $3.81 \mathrm{E}-01$ & $3.68 \mathrm{E}-09$ & $1.19 \mathrm{E}+01$ & 5.8 & 82.3 & 0 & $2.30 \mathrm{E}-04$ \\
\hline Co-60 & $1.92 \mathrm{E}+03$ & $7.40 \mathrm{E}-06$ & $1.19 \mathrm{E}+01$ & 27.6 & 60.1 & 0.2 & 4.69E-01 \\
\hline Co-60m & 7.27E-03 & $3.81 \mathrm{E}-11$ & $1.99 \mathrm{E}+01$ & 4 & 44.4 & 31.8 & 1.72E-06 \\
\hline Co-61 & $6.88 \mathrm{E}-02$ & $9.58 \mathrm{E}-10$ & $5.13 \mathrm{E}+01$ & 0 & 5.6 & 43.1 & 1.88E-05 \\
\hline Co-62m & $9.66 \mathrm{E}-03$ & $1.19 \mathrm{E}-10$ & $1.00 \mathrm{E}+02$ & 0 & 0 & 0 & $2.40 \mathrm{E}-07$ \\
\hline $\mathrm{Ni}-56$ & $6.10 \mathrm{E}+00$ & $3.08 \mathrm{E}-07$ & $7.30 \mathrm{E}+00$ & 9.4 & 80.5 & 2.9 & 1.94E-02 \\
\hline $\mathrm{Ni}-57$ & $1.50 \mathrm{E}+00$ & $4.64 \mathrm{E}-08$ & $2.15 \mathrm{E}+01$ & 7.2 & 49.6 & 21.8 & $2.75 \mathrm{E}-03$ \\
\hline $\mathrm{Ni}-59$ & $2.74 \mathrm{E}+07$ & $3.29 \mathrm{E}-08$ & $1.25 \mathrm{E}+01$ & 84.8 & 2.7 & 0 & 2.09E-03 \\
\hline $\mathrm{Ni}-63$ & $3.65 \mathrm{E}+04$ & $8.58 \mathrm{E}-08$ & $1.31 \mathrm{E}+01$ & 86.9 & 0 & 0 & 5.44E-03 \\
\hline $\mathrm{Ni}-65$ & $1.05 \mathrm{E}-01$ & $4.12 \mathrm{E}-09$ & $2.33 \mathrm{E}+01$ & 0 & 10 & 66.7 & $1.12 \mathrm{E}-04$ \\
\hline Cu-61 & $1.42 \mathrm{E}-01$ & 5.81E-09 & $1.38 \mathrm{E}+01$ & 0 & 16.6 & 69.5 & 1.91E-04 \\
\hline Cu-62 & $6.76 \mathrm{E}-03$ & $2.83 \mathrm{E}-09$ & $5.90 \mathrm{E}+00$ & 0 & 1.1 & 92.9 & $2.77 \mathrm{E}-06$ \\
\hline Cu-64 & 5.29E-01 & 3.20E-09 & $4.19 \mathrm{E}+01$ & 2 & 26.4 & 29.6 & 1.67E-04 \\
\hline Cu-66 & $3.54 \mathrm{E}-03$ & $3.56 \mathrm{E}-11$ & $1.00 \mathrm{E}+02$ & 0 & 0 & 0 & 4.12E-09 \\
\hline Cu-67 & $2.58 \mathrm{E}+00$ & $1.21 \mathrm{E}-08$ & $5.35 \mathrm{E}+01$ & 18.5 & 23.1 & 4.9 & $7.37 \mathrm{E}-04$ \\
\hline $\mathrm{Zn}-62$ & 3.86E-01 & 2.04E-08 & $5.20 \mathrm{E}+01$ & 1.3 & 23.5 & 23 & 1.13E-03 \\
\hline Zn-63 & $2.65 \mathrm{E}-02$ & $3.53 \mathrm{E}-10$ & $1.00 \mathrm{E}+02$ & 0 & 0 & 0 & 2.74E-06 \\
\hline $\mathrm{Zn}-65$ & $2.44 \mathrm{E}+02$ & 9.84E-06 & $1.10 \mathrm{E}+00$ & 91.7 & 7.3 & 0 & 6.23E-01 \\
\hline Zn-69 & 3.86E-02 & $1.89 \mathrm{E}-10$ & $1.00 \mathrm{E}+02$ & 0 & 0 & 0 & $2.18 \mathrm{E}-06$ \\
\hline $\mathrm{Zn}-69 \mathrm{~m}$ & $5.73 \mathrm{E}-01$ & 8.48E-09 & $4.79 \mathrm{E}+01$ & 3.5 & 24.3 & 24.4 & 4.58E-04 \\
\hline Zn-71m & $1.63 \mathrm{E}-01$ & 1.72E-09 & $1.00 \mathrm{E}+02$ & 0.1 & 0 & 0 & $6.11 \mathrm{E}-05$ \\
\hline $\mathrm{Zn}-72$ & $1.94 \mathrm{E}+00$ & $7.80 \mathrm{E}-08$ & $3.18 \mathrm{E}+01$ & 15.9 & 52.2 & 0.1 & 5.11E-03 \\
\hline Ga-66 & 3.92E-01 & $2.15 \mathrm{E}-08$ & $0.00 \mathrm{E}+00$ & 1.5 & 33.3 & 65.1 & 1.05E-03 \\
\hline Ga-67 & $3.26 \mathrm{E}+00$ & 9.59E-09 & $2.94 \mathrm{E}+01$ & 16.9 & 46 & 7.7 & 5.88E-04 \\
\hline Ga-68 & $4.72 \mathrm{E}-02$ & 5.22E-09 & $9.60 \mathrm{E}+00$ & 0 & 6.7 & 83.7 & 7.31E-05 \\
\hline
\end{tabular}


Table 5. Effective Dose for Annual Average Releases (1 TBq/a) of fusion activation products (continued)

\begin{tabular}{|c|c|c|c|c|c|c|c|}
\hline Nuclide & $\begin{array}{c}\text { Nuclide } \\
\text { Half-life } \\
\text { (days) }\end{array}$ & $\begin{array}{l}\text { MEI 2-km } \\
\text { total EDE } \\
\text { (Sv/year) }\end{array}$ & $\begin{array}{l}\text { Inhalation } \\
\% \text { of } \\
\text { total dose }\end{array}$ & $\begin{array}{l}\text { Ingestion } \\
\% \text { of } \\
\text { total dose }\end{array}$ & $\begin{array}{c}\text { Ground } \\
\text { surfaces } \\
\% \text { of } \\
\text { total dose }\end{array}$ & $\begin{array}{c}\text { Air } \\
\text { immersion } \\
\% \text { of } \\
\text { total dose }\end{array}$ & $\begin{array}{c}2-100 \mathrm{~km} \\
\text { population } \\
\text { dose } \\
\text { (person- } \\
\text { Sv/year) }\end{array}$ \\
\hline Ga-70 & $1.47 \mathrm{E}-02$ & $1.09 \mathrm{E}-10$ & $1.00 \mathrm{E}+02$ & 0 & 0 & 0 & $4.17 \mathrm{E}-07$ \\
\hline Ga-72 & $5.88 \mathrm{E}-01$ & $3.72 \mathrm{E}-08$ & $2.67 \mathrm{E}+01$ & 0 & 33.1 & 40.3 & $1.98 \mathrm{E}-03$ \\
\hline Ga-73 & $2.03 \mathrm{E}-01$ & 1.91E-09 & $9.95 \mathrm{E}+01$ & 0.4 & 0 & 0 & $7.52 \mathrm{E}-05$ \\
\hline Ga-74 & $5.63 \mathrm{E}-03$ & $0.00 \mathrm{E}+00$ & - & - & - & - & $0.00 \mathrm{E}+00$ \\
\hline Ga-76 & $3.14 \mathrm{E}-04$ & $0.00 \mathrm{E}+00$ & - & - & - & - & $0.00 \mathrm{E}+00$ \\
\hline Ge-68 & $2.88 \mathrm{E}+02$ & $6.06 \mathrm{E}-06$ & $4.80 \mathrm{E}+00$ & 72.8 & 22.4 & 0 & $3.85 \mathrm{E}-01$ \\
\hline Ge-69 & $1.63 E+00$ & $6.35 \mathrm{E}-09$ & $5.73 \mathrm{E}+01$ & 42.7 & 0 & 0 & $3.77 \mathrm{E}-04$ \\
\hline Ge-71 & $1.18 \mathrm{E}+01$ & $2.72 \mathrm{E}-09$ & $2.60 \mathrm{E}+01$ & 69.9 & 4.2 & 0 & $1.71 \mathrm{E}-04$ \\
\hline Ge-75 & $5.75 \mathrm{E}-02$ & $3.38 \mathrm{E}-10$ & $1.00 \mathrm{E}+02$ & 0 & 0 & 0 & $5.69 \mathrm{E}-06$ \\
\hline Ge-77 & $4.71 \mathrm{E}-01$ & $1.50 \mathrm{E}-08$ & $3.47 \mathrm{E}+01$ & 0.9 & 28.1 & 36.4 & $7.88 \mathrm{E}-04$ \\
\hline Ge-77m & $6.29 \mathrm{E}-04$ & $6.06 \mathrm{E}-12$ & $5.36 \mathrm{E}+01$ & 0.7 & 20.1 & 25.6 & $3.30 \mathrm{E}-07$ \\
\hline Ge-78 & $6.04 \mathrm{E}-02$ & $1.40 \mathrm{E}-09$ & $1.00 \mathrm{E}+02$ & 0 & 0 & 0 & $4.43 \mathrm{E}-05$ \\
\hline As-72 & $1.08 \mathrm{E}+00$ & 4.90E-08 & $4.18 \mathrm{E}+01$ & 6.3 & 32.9 & 18.8 & $2.82 \mathrm{E}-03$ \\
\hline As-73 & $8.03 E+01$ & $5.23 \mathrm{E}-08$ & $3.50 \mathrm{E}+01$ & 53.3 & 11.7 & 0 & $3.31 \mathrm{E}-03$ \\
\hline As-74 & $1.78 \mathrm{E}+01$ & $1.97 \mathrm{E}-07$ & $1.94 \mathrm{E}+01$ & 19.6 & 58.9 & 2 & $1.24 \mathrm{E}-02$ \\
\hline As-76 & $1.10 \mathrm{E}+00$ & $2.87 \mathrm{E}-08$ & $6.97 \mathrm{E}+01$ & 9.5 & 13.5 & 7.7 & $1.66 \mathrm{E}-03$ \\
\hline As-77 & $1.62 \mathrm{E}+00$ & 5.99E-09 & $9.72 \mathrm{E}+01$ & 0.1 & 2.1 & 0.7 & $3.56 \mathrm{E}-04$ \\
\hline As-78 & $6.29 \mathrm{E}-02$ & $1.26 \mathrm{E}-09$ & $1.00 \mathrm{E}+02$ & 0 & 0 & 0 & $2.30 \mathrm{E}-05$ \\
\hline As-79 & $6.25 \mathrm{E}-03$ & $9.05 \mathrm{E}-17$ & $4.30 \mathrm{E}+00$ & 95.7 & 0 & 0 & $1.97 \mathrm{E}-11$ \\
\hline As-80 & 1.91E-04 & - & - & - & - & - & $0.00 \mathrm{E}+00$ \\
\hline As- 81 & $3.70 \mathrm{E}-04$ & $2.60 \mathrm{E}-12$ & $1.00 \mathrm{E}+02$ & 0 & 0 & 0 & 8.26E-09 \\
\hline Se-73 & $2.98 \mathrm{E}-01$ & $1.01 \mathrm{E}-08$ & $1.84 \mathrm{E}+01$ & 0.4 & 28 & 53.6 & 4.62E-04 \\
\hline Se-75 & $1.20 \mathrm{E}+02$ & $1.50 \mathrm{E}-06$ & 3.2 & 71.3 & 25.1 & 0.1 & $9.48 \mathrm{E}-02$ \\
\hline Se-79 & $2.37 \mathrm{E}+07$ & $1.79 \mathrm{E}-06$ & 2.9 & 97.2 & 0 & 0 & 1.14E-01 \\
\hline Se-79m & $2.70 \mathrm{E}-03$ & $4.23 \mathrm{E}-17$ & 11.4 & 88.4 & 0 & 0 & $1.13 \mathrm{E}-11$ \\
\hline Se-81 & $1.29 \mathrm{E}-02$ & $8.74 \mathrm{E}-11$ & 100 & 0 & 0 & 0 & $2.79 \mathrm{E}-07$ \\
\hline Se-81m & $3.98 \mathrm{E}-02$ & $4.08 \mathrm{E}-10$ & 100 & 0 & 0 & 0 & $5.95 \mathrm{E}-06$ \\
\hline Se-83 & $1.56 \mathrm{E}-02$ & $2.15 \mathrm{E}-10$ & 90.2 & 0 & 9.2 & 0.7 & $3.31 \mathrm{E}-06$ \\
\hline Se- $83 m$ & $8.10 \mathrm{E}-04$ & $4.79 \mathrm{E}-12$ & 73.1 & 0 & 20.8 & 6.1 & $1.26 \mathrm{E}-07$ \\
\hline Se-84 & $2.29 \mathrm{E}-03$ & 1.38E-09 & 2.9 & 0 & 39.1 & 58.3 & 9.96E-06 \\
\hline $\mathrm{Br}-77$ & $2.33 \mathrm{E}+00$ & 1.08E-08 & 11.4 & 14.2 & 59.4 & 14.8 & $6.54 \mathrm{E}-04$ \\
\hline $\mathrm{Br}-80$ & 1.21E-02 & $4.80 \mathrm{E}-10$ & 22.9 & 0 & 22.1 & 55 & $1.40 \mathrm{E}-06$ \\
\hline $\mathrm{Br}-80 \mathrm{~m}$ & $1.84 \mathrm{E}-01$ & 4.65E-09 & 35.7 & 0.5 & 60.6 & 3.3 & 1.93E-04 \\
\hline $\mathrm{Br}-82$ & $1.47 \mathrm{E}+00$ & $7.05 \mathrm{E}-07$ & 1 & 7.5 & 89.5 & 1.9 & $4.16 \mathrm{E}-02$ \\
\hline $\mathrm{Br}-82 \mathrm{~m}$ & 4.26E-03 & 1.92E-09 & 0.7 & 5.2 & 93.2 & 1.3 & $1.18 \mathrm{E}-04$ \\
\hline $\mathrm{Br}-83$ & 9.96E-02 & $5.85 \mathrm{E}-10$ & 73.2 & 0 & 20.7 & 6.2 & $1.54 \mathrm{E}-05$ \\
\hline $\mathrm{Br}-84$ & $2.21 \mathrm{E}-02$ & 1.36E-08 & 3.1 & 0 & 34.9 & 61.9 & $8.60 \mathrm{E}-05$ \\
\hline $\mathrm{Br}-84 \mathrm{~m}$ & 4.17E-03 & $0.00 \mathrm{E}+00$ & - & - & - & - & $0.00 \mathrm{E}+00$ \\
\hline $\mathrm{Br}-85$ & 1.99E-03 & $4.73 \mathrm{E}-11$ & 0 & 0 & 4.8 & 95.1 & $3.27 \mathrm{E}-07$ \\
\hline $\mathrm{Br}-86$ & $6.37 \mathrm{E}-04$ & $0.00 \mathrm{E}+00$ & - & - & - & - & $0.00 \mathrm{E}+00$ \\
\hline $\mathrm{Br}-87$ & $6.46 \mathrm{E}-04$ & $5.09 \mathrm{E}-11$ & 0 & 0 & 0 & 100 & $7.96 \mathrm{E}-07$ \\
\hline $\mathrm{Br}-88$ & $1.89 \mathrm{E}-04$ & $2.03 \mathrm{E}-11$ & 1.1 & 0 & 4.4 & 94.6 & 7.37E-07 \\
\hline Kr-79 & $1.46 \mathrm{E}+00$ & $1.28 \mathrm{E}-09$ & 0 & 0 & 0 & 100 & $7.54 \mathrm{E}-05$ \\
\hline $\mathrm{Kr}-81$ & $7.67 \mathrm{E}+07$ & $5.15 \mathrm{E}-11$ & 0 & 0 & 0 & 100 & $3.27 E-06$ \\
\hline
\end{tabular}


Table 5. Effective Dose for Annual Average Releases (1 TBq/a) of fusion activation products (continued)

\begin{tabular}{|c|c|c|c|c|c|c|c|}
\hline Nuclide & $\begin{array}{c}\text { Nuclide } \\
\text { Half-life } \\
\text { (days) }\end{array}$ & $\begin{array}{l}\text { MEI 2-km } \\
\text { total EDE } \\
\text { (Sv/year) }\end{array}$ & $\begin{array}{l}\text { Inhalation } \\
\% \text { of } \\
\text { total dose }\end{array}$ & $\begin{array}{l}\text { Ingestion } \\
\% \text { of } \\
\text { total dose }\end{array}$ & $\begin{array}{c}\text { Ground } \\
\text { surfaces } \\
\% \text { of } \\
\text { total dose }\end{array}$ & $\begin{array}{c}\text { Air } \\
\text { immersion } \\
\% \text { of } \\
\text { total dose }\end{array}$ & $\begin{array}{c}2-100 \mathrm{~km} \\
\text { population } \\
\text { dose } \\
\text { (person- } \\
\text { Sv/year) }\end{array}$ \\
\hline Kr-83m & $7.63 \mathrm{E}-02$ & $4.47 \mathrm{E}-13$ & 0 & 0 & 0 & 100 & $9.58 \mathrm{E}-09$ \\
\hline $\mathrm{Kr}-85$ & $3.91 \mathrm{E}+03$ & $1.13 \mathrm{E}-11$ & 0 & 0 & 0 & 100 & $7.17 \mathrm{E}-07$ \\
\hline $\mathrm{Kr}-85 \mathrm{~m}$ & $1.87 \mathrm{E}-01$ & $8.01 \mathrm{E}-10$ & 0 & 0 & 0 & 100 & $3.04 \mathrm{E}-05$ \\
\hline $\mathrm{Kr}-87$ & $5.30 \mathrm{E}-02$ & $4.13 \mathrm{E}-09$ & 0 & 0 & 0 & 100 & $6.45 \mathrm{E}-05$ \\
\hline $\mathrm{Kr}-88$ & $1.18 \mathrm{E}-01$ & $1.28 \mathrm{E}-08$ & 1.1 & 0 & 4.4 & 94.5 & $4.62 \mathrm{E}-04$ \\
\hline $\mathrm{Kr}-89$ & $2.19 \mathrm{E}-03$ & $2.97 \mathrm{E}-09$ & 1 & 0 & 0.9 & 98 & $5.32 \mathrm{E}-06$ \\
\hline $\mathrm{Kr}-90$ & $3.74 \mathrm{E}-04$ & $3.20 \mathrm{E}-10$ & 0 & 0.2 & 0.2 & 99.7 & $4.95 \mathrm{E}-08$ \\
\hline Kr-92 & $2.13 \mathrm{E}-05$ & $2.11 \mathrm{E}-12$ & 28.3 & 0 & 11.6 & 60.2 & $7.62 \mathrm{E}-08$ \\
\hline $\mathrm{Kr}-93$ & 1.47E-05 & $1.50 \mathrm{E}-11$ & 1.6 & 0 & 0.8 & 97.3 & $2.99 \mathrm{E}-08$ \\
\hline Kr-95 & $5.79 \mathrm{E}-06$ & $1.89 \mathrm{E}-13$ & 52.1 & 10.3 & 37.5 & 0.1 & 7.06E-09 \\
\hline $\mathrm{Rb}-81$ & 1.91E-01 & $4.57 \mathrm{E}-09$ & 12.6 & 0 & 21.6 & 65.9 & $1.75 \mathrm{E}-04$ \\
\hline $\mathrm{Rb}-82$ & $9.03 \mathrm{E}-04$ & $3.97 \mathrm{E}-11$ & 0 & 0 & 0.2 & 99.7 & $0.00 \mathrm{E}+00$ \\
\hline $\mathrm{Rb}-83$ & $8.62 \mathrm{E}+01$ & 1.39E-06 & 2.1 & 72.7 & 25.2 & 0.2 & $8.83 \mathrm{E}-02$ \\
\hline $\mathrm{Rb}-84$ & $3.28 \mathrm{E}+01$ & $8.52 \mathrm{E}-07$ & 4.5 & 66.2 & 28.8 & 0.5 & $5.39 \mathrm{E}-02$ \\
\hline $\mathrm{Rb}-86$ & $1.87 \mathrm{E}+01$ & $3.31 \mathrm{E}-07$ & 11.8 & 83.7 & 4.2 & 0.2 & $2.08 \mathrm{E}-02$ \\
\hline $\mathrm{Rb}-87$ & $1.73 \mathrm{E}+13$ & 1.47E-06 & 1.3 & 98.6 & 0 & 0 & $9.30 \mathrm{E}-02$ \\
\hline $\mathrm{Rb}-88$ & $1.24 \mathrm{E}-02$ & $2.88 \mathrm{E}-09$ & 11.4 & 0 & 1.4 & 87.2 & $8.70 \mathrm{E}-06$ \\
\hline $\mathrm{Rb}-89$ & $1.06 \mathrm{E}-02$ & $7.74 \mathrm{E}-09$ & 1.9 & 0.1 & 1.4 & 96.6 & $2.08 \mathrm{E}-05$ \\
\hline $\mathrm{Rb}-90$ & $1.77 \mathrm{E}-03$ & 1.05E-09 & 0 & 0.2 & 0.2 & 99 & $2.16 \mathrm{E}-07$ \\
\hline $\mathrm{Rb}-90 \mathrm{~m}$ & $2.99 \mathrm{E}-03$ & $4.32 \mathrm{E}-09$ & 0 & 0.1 & 0.4 & 99.5 & $5.82 \mathrm{E}-07$ \\
\hline $\mathrm{Rb}-91$ & $6.74 \mathrm{E}-04$ & $2.07 \mathrm{E}-11$ & 40.4 & 2.5 & 26.6 & 30.6 & $1.18 \mathrm{E}-06$ \\
\hline $\mathrm{Rb}-92$ & $5.19 \mathrm{E}-05$ & $5.14 \mathrm{E}-12$ & 28.2 & 0 & 11.6 & 60.1 & $1.86 \mathrm{E}-07$ \\
\hline $\mathrm{Rb}-93$ & 6.71E-05 & $6.84 \mathrm{E}-11$ & 1.6 & 0 & 0.8 & 97.5 & $1.37 \mathrm{E}-07$ \\
\hline $\mathrm{Rb}-95$ & $4.17 \mathrm{E}-06$ & $1.36 \mathrm{E}-13$ & 52.1 & 10.3 & 37.5 & 0.1 & 5.08E-09 \\
\hline Sr-82 & $2.50 \mathrm{E}+01$ & $2.39 \mathrm{E}-07$ & 0 & 0 & 97.5 & 2.3 & $1.51 \mathrm{E}-02$ \\
\hline Sr-85 & $6.48 \mathrm{E}+01$ & $3.37 \mathrm{E}-07$ & 3.3 & 13 & 82.8 & 0.8 & $2.13 \mathrm{E}-02$ \\
\hline Sr-85m & $4.83 \mathrm{E}-02$ & 1.32E-09 & 3.4 & 0.3 & 20.5 & 75.8 & 2.97E-05 \\
\hline $\mathrm{Sr}-87 \mathrm{~m}$ & 1.17E-01 & $2.08 \mathrm{E}-09$ & 10.4 & 0 & 15.2 & 74.5 & $6.08 \mathrm{E}-05$ \\
\hline Sr-89 & $5.05 \mathrm{E}+01$ & $1.99 \mathrm{E}-07$ & 17.5 & 82.4 & 0 & 0 & $1.26 \mathrm{E}-02$ \\
\hline Sr-90 & $1.06 \mathrm{E}+04$ & $2.03 \mathrm{E}-05$ & 6.7 & 93.6 & 0 & 0 & $1.29 \mathrm{E}+00$ \\
\hline Sr-91 & $3.96 \mathrm{E}-01$ & $1.22 \mathrm{E}-08$ & 40.3 & 2.5 & 26.5 & 30.7 & $6.90 \mathrm{E}-04$ \\
\hline Sr-92 & $1.13 \mathrm{E}-01$ & 1.12E-08 & 28.3 & 0 & 11.6 & 60.1 & $4.04 \mathrm{E}-04$ \\
\hline Sr-93 & $5.21 \mathrm{E}-03$ & 5.24E-09 & 1.7 & 0 & 0.8 & 97.5 & $1.06 \mathrm{E}-05$ \\
\hline Sr-94 & $8.75 \mathrm{E}-04$ & 1.97E-11 & 100 & 0 & 0 & 0 & 6.63E-08 \\
\hline Sr-95 & $3.01 \mathrm{E}-04$ & $9.81 \mathrm{E}-12$ & 52.1 & 10.3 & 37.5 & 0.1 & $3.67 \mathrm{E}-07$ \\
\hline Sr-97 & 2.32E-06 & 1.15E-13 & 60.3 & 0 & 25.8 & 13.7 & 6.83E-09 \\
\hline Y-86 & $6.14 \mathrm{E}-01$ & $4.57 \mathrm{E}-08$ & 20.5 & 0 & 37.9 & 41.8 & $2.45 \mathrm{E}-03$ \\
\hline Y-87 & $3.35 \mathrm{E}+00$ & $3.95 \mathrm{E}-08$ & 23.8 & 12.8 & 57.5 & 6 & $2.50 \mathrm{E}-03$ \\
\hline Y-88 & $1.07 \mathrm{E}+02$ & 2.37E-06 & 5.2 & 10.3 & 84 & 0.6 & $1.51 \mathrm{E}-01$ \\
\hline Y-90 & $2.67 \mathrm{E}+00$ & $6.63 \mathrm{E}-08$ & 72.7 & 27.3 & 0 & 0 & $4.04 \mathrm{E}-03$ \\
\hline Y-90m & $1.33 \mathrm{E}-01$ & $6.45 \mathrm{E}-09$ & 40.2 & 0.6 & 11.1 & 48.2 & $3.07 \mathrm{E}-04$ \\
\hline Y-91 & $5.85 \mathrm{E}+01$ & 5.66E-07 & 45.8 & 53.9 & 0.3 & 0 & $3.59 \mathrm{E}-02$ \\
\hline $\mathrm{Y}-91 \mathrm{~m}$ & $3.45 \mathrm{E}-02$ & 2.71E-09 & 6.6 & 1 & 5.1 & 87.5 & $4.51 \mathrm{E}-05$ \\
\hline $\mathrm{Y}-92$ & $1.48 \mathrm{E}-01$ & 5.11E-09 & 69.3 & 0 & 5.6 & 25 & $1.72 \mathrm{E}-04$ \\
\hline
\end{tabular}


Table 5. Effective Dose for Annual Average Releases (1 TBq/a) of fusion activation products (continued)

\begin{tabular}{|c|c|c|c|c|c|c|c|}
\hline Nuclide & $\begin{array}{c}\text { Nuclide } \\
\text { Half-life } \\
\text { (days) }\end{array}$ & $\begin{array}{l}\text { MEI 2-km } \\
\text { total EDE } \\
\text { (Sv/year) }\end{array}$ & $\begin{array}{l}\text { Inhalation } \\
\% \text { of } \\
\text { total dose }\end{array}$ & $\begin{array}{l}\text { Ingestion } \\
\% \text { of } \\
\text { total dose }\end{array}$ & $\begin{array}{c}\text { Ground } \\
\text { surfaces } \\
\% \text { of } \\
\text { total dose }\end{array}$ & $\begin{array}{c}\text { Air } \\
\text { immersion } \\
\% \text { of } \\
\text { total dose }\end{array}$ & $\begin{array}{c}2-100 \mathrm{~km} \\
\text { population } \\
\text { dose } \\
\text { (person- } \\
\text { Sv/year) }\end{array}$ \\
\hline Y-93 & $4.21 \mathrm{E}-01$ & 1.30E-08 & 93.8 & 0 & 2.3 & 3.7 & $6.48 \mathrm{E}-04$ \\
\hline Y-94 & 1.33E-02 & $2.82 \mathrm{E}-10$ & 100 & 0 & 0 & 0 & $9.44 \mathrm{E}-07$ \\
\hline Y-95 & $7.29 \mathrm{E}-03$ & $2.33 \mathrm{E}-10$ & 51.1 & 10.5 & 38.3 & 0.1 & $8.90 \mathrm{E}-06$ \\
\hline Y-97 & $1.29 \mathrm{E}-05$ & $6.37 \mathrm{E}-13$ & 60.4 & 0 & 25.9 & 13.6 & $3.79 \mathrm{E}-08$ \\
\hline Zr-86 & $6.88 \mathrm{E}-01$ & $3.49 \mathrm{E}-08$ & 32.1 & 2.5 & 61 & 4.4 & $2.31 \mathrm{E}-03$ \\
\hline Zr-88 & $8.34 \mathrm{E}+01$ & 1.71E-06 & 3.4 & 5.8 & 90.6 & 0.1 & $1.09 \mathrm{E}-01$ \\
\hline Zr-89 & $3.27 \mathrm{E}+00$ & $5.60 \mathrm{E}-08$ & 21.1 & 12.5 & 55.7 & 10.8 & $3.44 \mathrm{E}-03$ \\
\hline Zr-93 & $5.58 \mathrm{E}+08$ & $6.46 \mathrm{E}-07$ & 73.8 & 26.2 & 0 & 0 & $4.10 \mathrm{E}-02$ \\
\hline Zr-95 & $6.40 \mathrm{E}+01$ & $1.21 \mathrm{E}-06$ & 6.3 & 29.1 & 64.6 & 0.3 & 7.70E-02 \\
\hline Zr-97 & $7.04 \mathrm{E}-01$ & $3.49 \mathrm{E}-08$ & 60.5 & 0 & 25.9 & 13.6 & $2.08 \mathrm{E}-03$ \\
\hline Zr-99 & $2.78 \mathrm{E}-05$ & $2.98 \mathrm{E}-13$ & 71.8 & 3.1 & 22.3 & 2.8 & $1.84 \mathrm{E}-08$ \\
\hline $\mathrm{Nb}-90$ & $6.08 \mathrm{E}-01$ & $5.67 \mathrm{E}-08$ & 21.7 & 3.2 & 33.9 & 41.3 & $3.03 \mathrm{E}-03$ \\
\hline $\mathrm{Nb}-92 \mathrm{~m}$ & $1.02 \mathrm{E}+01$ & $2.20 \mathrm{E}-07$ & 5.9 & 55.5 & 36.3 & 2.3 & $1.38 \mathrm{E}-02$ \\
\hline $\mathrm{Nb}-93 \mathrm{~m}$ & $4.96 \mathrm{E}+03$ & $1.11 \mathrm{E}-06$ & 14.9 & 84.7 & 0.2 & 0 & $7.02 \mathrm{E}-02$ \\
\hline $\mathrm{Nb}-94$ & $7.41 \mathrm{E}+06$ & $1.45 \mathrm{E}-05$ & 13.4 & 63.6 & 22.9 & 0.1 & $9.19 \mathrm{E}-01$ \\
\hline $\mathrm{Nb}-94 \mathrm{~m}$ & $4.35 \mathrm{E}-03$ & $1.15 \mathrm{E}-11$ & 23 & 0 & 0.9 & 76.2 & $3.49 \mathrm{E}-09$ \\
\hline $\mathrm{Nb}-95$ & $3.51 \mathrm{E}+01$ & 9.74E-07 & 2.7 & 73.7 & 23.1 & 0.4 & $6.16 \mathrm{E}-02$ \\
\hline $\mathrm{Nb}-95 \mathrm{~m}$ & $3.61 \mathrm{E}+00$ & $6.56 \mathrm{E}-08$ & 19.7 & 43.3 & 36.4 & 0.5 & $4.10 \mathrm{E}-03$ \\
\hline $\mathrm{Nb}-96$ & $9.73 \mathrm{E}-01$ & $4.90 \mathrm{E}-08$ & 23.9 & 10 & 40 & 26.1 & $2.79 \mathrm{E}-03$ \\
\hline $\mathrm{Nb}-97$ & $5.01 \mathrm{E}-02$ & $3.78 \mathrm{E}-09$ & 10.1 & 0 & 6.8 & 83.1 & $5.60 \mathrm{E}-05$ \\
\hline $\mathrm{Nb}-97 \mathrm{~m}$ & $6.94 \mathrm{E}-04$ & $5.93 \mathrm{E}-11$ & 9.1 & 0 & 6.1 & 84.8 & $7.87 \mathrm{E}-07$ \\
\hline $\mathrm{Nb}-98$ & $3.58 \mathrm{E}-02$ & $5.20 \mathrm{E}-10$ & 100 & 0 & 0 & 0 & 5.53E-06 \\
\hline $\mathrm{Nb}-98$ & 3.24E-05 & $5.20 \mathrm{E}-10$ & 100 & 0 & 0 & 0 & 5.53E-06 \\
\hline $\mathrm{Nb}-99$ & $1.66 \mathrm{E}-04$ & $2.10 \mathrm{E}-12$ & 60.5 & 18.1 & 18.9 & 2.3 & $1.29 \mathrm{E}-07$ \\
\hline $\mathrm{Nb}-99 \mathrm{~m}$ & $1.81 \mathrm{E}-03$ & $2.14 \mathrm{E}-11$ & 59.3 & 17.9 & 20.2 & 2.3 & $1.41 \mathrm{E}-06$ \\
\hline $\mathrm{Nb}-101$ & $8.10 \mathrm{E}-05$ & $4.74 \mathrm{E}-11$ & 2.5 & 0 & 1.8 & 95.8 & $1.40 \mathrm{E}-07$ \\
\hline Mo-93 & $1.28 \mathrm{E}+06$ & 3.74E-07 & 44.1 & 52.7 & 3.3 & 0 & $2.38 \mathrm{E}-02$ \\
\hline Mo-93m & $2.85 E-01$ & $2.10 \mathrm{E}-09$ & 99.5 & 0.6 & 0 & 0 & $9.40 \mathrm{E}-05$ \\
\hline Mo-99 & $2.75 \mathrm{E}+00$ & $3.49 \mathrm{E}-08$ & 60.7 & 18.2 & 18.9 & 2.3 & $2.15 \mathrm{E}-03$ \\
\hline Mo-101 & 1.02E-02 & $5.90 \mathrm{E}-09$ & 2.5 & 0 & 1.8 & 95.8 & $1.74 \mathrm{E}-05$ \\
\hline Tc-95 & 8.33E-01 & 1.14E-08 & 15 & 0.9 & 48.2 & 35.9 & $6.37 \mathrm{E}-04$ \\
\hline Tc-95m & $6.10 \mathrm{E}+01$ & $3.63 \mathrm{E}-07$ & 0 & 0 & 99.2 & 0.9 & $2.30 \mathrm{E}-02$ \\
\hline Tc-96 & $4.28 \mathrm{E}+00$ & 1.34E-07 & 10.5 & 13.6 & 66.3 & 9.8 & 8.30E-03 \\
\hline Tc-96m & $3.58 \mathrm{E}-02$ & 1.09E-09 & 11.3 & 2.2 & 67.8 & 18.8 & $6.86 \mathrm{E}-05$ \\
\hline Tc-97 & $9.49 \mathrm{E}+08$ & $2.96 \mathrm{E}-07$ & 1.8 & 93.6 & 4.5 & 0 & 1.87E-02 \\
\hline Tc-97m & $8.70 \mathrm{E}+01$ & $1.78 \mathrm{E}-07$ & 13.9 & 83.7 & 2.1 & 0 & 1.13E-02 \\
\hline Tc-98 & $1.53 \mathrm{E}+09$ & $1.20 \mathrm{E}-05$ & 0.8 & 73.8 & 24.9 & 0.1 & $7.59 \mathrm{E}-01$ \\
\hline Tc-99 & $7.78 \mathrm{E}+07$ & $2.44 \mathrm{E}-06$ & 1.8 & 98.4 & 0 & 0 & $1.55 \mathrm{E}-01$ \\
\hline Tc-99m & $2.51 \mathrm{E}-01$ & 1.07E-09 & 11.4 & 0 & 27.9 & 61 & $4.59 \mathrm{E}-05$ \\
\hline Tc-101 & 9.86E-03 & 1.17E-09 & 4.2 & 0 & 1.6 & 94.9 & $2.46 \mathrm{E}-06$ \\
\hline Tc-102 & $6.11 \mathrm{E}-05$ & - & - & - & - & - & $0.00 \mathrm{E}+00$ \\
\hline Tc-102m & $3.02 \mathrm{E}-03$ & $0.00 \mathrm{E}+00$ & - & - & - & - & $0.00 \mathrm{E}+00$ \\
\hline Tc-104 & $1.26 \mathrm{E}-02$ & $2.40 \mathrm{E}-10$ & 100 & 0 & 0 & 0 & 7.47E-07 \\
\hline Ru-97 & $2.90 \mathrm{E}+00$ & 1.11E-08 & 22.3 & 11.4 & 55.9 & 10.5 & 6.79E-04 \\
\hline
\end{tabular}


Table 5. Effective Dose for Annual Average Releases (1 TBq/a) of fusion activation products (continued)

\begin{tabular}{|c|c|c|c|c|c|c|c|}
\hline Nuclide & $\begin{array}{c}\text { Nuclide } \\
\text { Half-life } \\
\text { (days) }\end{array}$ & $\begin{array}{l}\text { MEI 2-km } \\
\text { total EDE } \\
\text { (Sv/year) }\end{array}$ & $\begin{array}{l}\text { Inhalation } \\
\% \text { of } \\
\text { total dose }\end{array}$ & $\begin{array}{l}\text { Ingestion } \\
\% \text { of } \\
\text { total dose }\end{array}$ & $\begin{array}{c}\text { Ground } \\
\text { surfaces } \\
\% \text { of } \\
\text { total dose }\end{array}$ & $\begin{array}{c}\text { Air } \\
\text { immersion } \\
\% \text { of } \\
\text { total dose }\end{array}$ & $\begin{array}{c}2-100 \mathrm{~km} \\
\text { population } \\
\text { dose } \\
\text { (person- } \\
\text { Sv/year) }\end{array}$ \\
\hline Ru-103 & $3.94 \mathrm{E}+01$ & $2.48 \mathrm{E}-07$ & 18.5 & 14.7 & 65.7 & 1 & $1.57 \mathrm{E}-02$ \\
\hline Ru-105 & $1.85 \mathrm{E}-01$ & 7.71E-09 & 30.7 & 0.1 & 17.4 & 51.8 & $3.13 \mathrm{E}-04$ \\
\hline Ru-106 & $3.68 \mathrm{E}+02$ & $3.47 \mathrm{E}-06$ & 74.6 & 16 & 9.2 & 0 & $2.20 \mathrm{E}-01$ \\
\hline Ru-108 & $3.13 \mathrm{E}-03$ & $0.00 \mathrm{E}+00$ & - & - & - & - & $0.00 \mathrm{E}+00$ \\
\hline Rh-101 & $1.17 \mathrm{E}+03$ & $5.88 \mathrm{E}-07$ & 32.1 & 68 & 0 & 0 & $3.73 \mathrm{E}-02$ \\
\hline Rh-101m & $4.34 \mathrm{E}+00$ & $9.73 \mathrm{E}-09$ & 40.5 & 59.5 & 0 & 0 & $6.03 \mathrm{E}-04$ \\
\hline Rh-102 & $1.06 \mathrm{E}+03$ & $1.98 \mathrm{E}-06$ & 25.9 & 74.2 & 0 & 0 & $1.25 \mathrm{E}-01$ \\
\hline Rh-102m & $2.07 \mathrm{E}+02$ & $7.22 \mathrm{E}-07$ & 34.2 & 65.7 & 0 & 0 & $4.57 \mathrm{E}-02$ \\
\hline Rh-103m & $3.90 \mathrm{E}-02$ & $2.33 \mathrm{E}-11$ & 94.8 & 0 & 1.4 & 3.9 & $2.70 \mathrm{E}-07$ \\
\hline Rh-105 & $1.47 \mathrm{E}+00$ & $7.73 \mathrm{E}-09$ & 67.7 & 14.1 & 13.2 & 5.1 & $4.56 \mathrm{E}-04$ \\
\hline Rh-105m & $5.21 \mathrm{E}-04$ & $2.21 \mathrm{E}-12$ & 67 & 13.9 & 13.1 & 6.3 & $1.29 \mathrm{E}-07$ \\
\hline Rh-106 & $3.46 \mathrm{E}-04$ & $2.70 \mathrm{E}-15$ & 0 & 0 & 0.1 & 100 & $0.00 \mathrm{E}+00$ \\
\hline Rh-106m & $9.17 \mathrm{E}-02$ & $8.42 \mathrm{E}-10$ & 100 & 0 & 0 & 0 & $2.08 \mathrm{E}-05$ \\
\hline Rh-107 & $1.51 \mathrm{E}-02$ & $8.76 \mathrm{E}-11$ & 100 & 0 & 0 & 0 & $3.48 \mathrm{E}-07$ \\
\hline Rh-108 & $1.94 \mathrm{E}-04$ & - & - & - & - & - & $0.00 \mathrm{E}+00$ \\
\hline Rh-108m & $4.10 \mathrm{E}-03$ & $0.00 \mathrm{E}+00$ & - & - & - & - & $0.00 \mathrm{E}+00$ \\
\hline $\mathrm{Pd}-103$ & $1.70 \mathrm{E}+01$ & $2.56 \mathrm{E}-08$ & 32.2 & 61.3 & 6.3 & 0 & $1.62 \mathrm{E}-03$ \\
\hline $\mathrm{Pd}-107$ & $2.37 \mathrm{E}+09$ & 1.07E-07 & 71.6 & 28.1 & 0 & 0 & $6.76 \mathrm{E}-03$ \\
\hline $\mathrm{Pd}-107 \mathrm{~m}$ & $2.47 \mathrm{E}-04$ & $1.11 \mathrm{E}-20$ & 71.6 & 28.2 & 0 & 0 & $7.02 \mathrm{E}-16$ \\
\hline Pd-109 & $5.60 \mathrm{E}-01$ & $6.64 \mathrm{E}-09$ & 96.8 & 2.1 & 0.7 & 0.4 & 3.51E-04 \\
\hline$P d-109 m$ & $3.26 \mathrm{E}-03$ & $2.89 \mathrm{E}-11$ & 96.9 & 2.1 & 0.9 & 0.4 & $2.05 E-06$ \\
\hline $\mathrm{Pd}-111$ & $1.53 \mathrm{E}-02$ & $4.54 \mathrm{E}-10$ & 89 & 10.3 & 0.8 & 0 & 1.54E-05 \\
\hline$P d-112$ & $8.38 \mathrm{E}-01$ & $1.09 \mathrm{E}-10$ & 100 & 0 & 0 & 0 & $9.75 \mathrm{E}-05$ \\
\hline $\mathrm{Ag}-106$ & $1.66 \mathrm{E}-02$ & $1.17 \mathrm{E}-10$ & 100 & 0 & 0 & 0 & 5.29E-07 \\
\hline $\mathrm{Ag}-106 \mathrm{~m}$ & $8.41 \mathrm{E}+00$ & $3.51 \mathrm{E}-07$ & 9.1 & 32.2 & 54.4 & 4.1 & $2.20 \mathrm{E}-02$ \\
\hline $\mathrm{Ag}-108$ & 1.65E-03 & $8.19 \mathrm{E}-12$ & 27.2 & 0 & 0.2 & 72.5 & 5.52E-12 \\
\hline $\mathrm{Ag}-108 \mathrm{~m}$ & $4.64 \mathrm{E}+04$ & $7.45 \mathrm{E}-06$ & 15.8 & 37.3 & 46.8 & 0.1 & 4.73E-01 \\
\hline $\mathrm{Ag}-109 \mathrm{~m}$ & $4.58 \mathrm{E}-04$ & $1.34 \mathrm{E}-15$ & 0 & 0 & 0.2 & 100 & $0.00 \mathrm{E}+00$ \\
\hline $\mathrm{Ag}-110$ & $2.84 \mathrm{E}-04$ & $2.60 \mathrm{E}-17$ & 6.6 & 0 & 0 & 93.1 & $0.00 \mathrm{E}+00$ \\
\hline $\mathrm{Ag}-110 \mathrm{~m}$ & $2.50 \mathrm{E}+02$ & $6.36 \mathrm{E}-06$ & 4.9 & 38.7 & 56.1 & 0.2 & 4.03E-01 \\
\hline $\mathrm{Ag}-111$ & $7.46 \mathrm{E}+00$ & $1.12 \mathrm{E}-07$ & 31 & 67.6 & 1.6 & 0.1 & 7.02E-03 \\
\hline $\mathrm{Ag}-111 \mathrm{~m}$ & 7.52E-04 & 1.13E-11 & 30.9 & 67.4 & 1.6 & 0.1 & 7.08E-07 \\
\hline $\mathrm{Ag}-112$ & 1.30E-01 & 3.13E-09 & 100 & 0 & 0 & 0 & 9.82E-05 \\
\hline $\mathrm{Ag}-114$ & 5.23E-05 & - & - & - & - & - & $0.00 \mathrm{E}+00$ \\
\hline $\mathrm{Ag}-115$ & 1.39E-02 & $2.61 \mathrm{E}-10$ & 100 & 0 & 0 & 0 & $9.29 \mathrm{E}-07$ \\
\hline Cd-109 & $4.64 \mathrm{E}+02$ & $1.23 \mathrm{E}-06$ & 20.1 & 78.4 & 1.2 & 0 & $7.78 \mathrm{E}-02$ \\
\hline $\mathrm{Cd}-111 \mathrm{~m}$ & 3.38E-02 & 1.33E-09 & 0 & 0 & 5.8 & 94.7 & $1.34 \mathrm{E}-05$ \\
\hline Cd-113 & $3.39 E+18$ & 2.51E-05 & 8.7 & 91.2 & 0 & 0 & $1.59 \mathrm{E}+00$ \\
\hline Cd-113m & $4.96 \mathrm{E}+03$ & $2.08 \mathrm{E}-05$ & 10.8 & 88.9 & 0 & 0 & $1.32 \mathrm{E}+00$ \\
\hline Cd-115 & $2.23 \mathrm{E}+00$ & 3.82E-08 & 58.6 & 20.1 & 18.5 & 2.7 & $2.36 \mathrm{E}-03$ \\
\hline Cd-115m & $4.46 \mathrm{E}+01$ & 6.89E-07 & 29.9 & 68.9 & 1.1 & 0 & $4.36 \mathrm{E}-02$ \\
\hline Cd-117 & $1.08 \mathrm{E}-01$ & 9.64E-09 & 21.4 & 0 & 17 & 61.6 & $3.72 \mathrm{E}-04$ \\
\hline $\mathrm{Cd}-117 \mathrm{~m}$ & 1.42E-01 & 1.51E-08 & 13 & 0 & 14.6 & 72.8 & $5.11 \mathrm{E}-04$ \\
\hline Cd-119m & $2.22 \mathrm{E}-03$ & $0.00 \mathrm{E}+00$ & 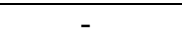 & - & 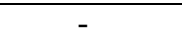 & - & $0.00 \mathrm{E}+00$ \\
\hline
\end{tabular}


Table 5. Effective Dose for Annual Average Releases (1 TBq/a) of fusion activation products (continued)

\begin{tabular}{|c|c|c|c|c|c|c|c|}
\hline Nuclide & $\begin{array}{c}\text { Nuclide } \\
\text { Half-life } \\
\text { (days) }\end{array}$ & $\begin{array}{l}\text { MEI 2-km } \\
\text { total EDE } \\
\text { (Sv/year) }\end{array}$ & $\begin{array}{l}\text { Inhalation } \\
\% \text { of } \\
\text { total dose }\end{array}$ & $\begin{array}{l}\text { Ingestion } \\
\% \text { of } \\
\text { total dose }\end{array}$ & $\begin{array}{c}\text { Ground } \\
\text { surfaces } \\
\% \text { of } \\
\text { total dose }\end{array}$ & $\begin{array}{c}\text { Air } \\
\text { immersion } \\
\% \text { of } \\
\text { total dose }\end{array}$ & $\begin{array}{c}2-100 \mathrm{~km} \\
\text { population } \\
\text { dose } \\
\text { (person- } \\
\text { Sv/year) }\end{array}$ \\
\hline $\ln -111$ & $2.83 E+00$ & 1.91E-08 & 23.4 & 12.3 & 53.9 & 10.5 & $1.17 \mathrm{E}-03$ \\
\hline $\ln -113 m$ & $6.91 \mathrm{E}-02$ & 1.49E-09 & 9.7 & 0 & 9.8 & 80.5 & $2.95 \mathrm{E}-05$ \\
\hline $\ln -114$ & $8.32 \mathrm{E}-04$ & $8.65 \mathrm{E}-13$ & 11.5 & 0 & 0.1 & 88.3 & $0.00 \mathrm{E}+00$ \\
\hline $\ln -114 m$ & $4.95 \mathrm{E}+01$ & $9.89 \mathrm{E}-07$ & 29.2 & 65.4 & 5.3 & 0.1 & $6.27 \mathrm{E}-02$ \\
\hline $\ln -115$ & $1.86 \mathrm{E}+18$ & $2.21 \mathrm{E}-05$ & 24.8 & 75.1 & 0 & 0 & $1.40 \mathrm{E}+00$ \\
\hline $\ln -115 \mathrm{~m}$ & $1.82 \mathrm{E}-01$ & 1.61E-09 & 35.7 & 0 & 15.8 & 48.6 & $6.03 \mathrm{E}-05$ \\
\hline $\ln -116 \mathrm{~m}$ & $3.76 \mathrm{E}-02$ & $1.24 \mathrm{E}-08$ & 1.7 & 0 & 5 & 93.5 & $1.39 \mathrm{E}-04$ \\
\hline $\ln -117$ & $3.04 \mathrm{E}-02$ & 3.32E-09 & 3.7 & 0 & 4.8 & 91.6 & $2.98 \mathrm{E}-05$ \\
\hline $\ln -117 m$ & $8.08 \mathrm{E}-02$ & $1.17 \mathrm{E}-09$ & 57.2 & 0 & 5.7 & 36.8 & $2.69 \mathrm{E}-05$ \\
\hline $\ln -118$ & $5.79 \mathrm{E}-05$ & - & - & - & - & - & $0.00 \mathrm{E}+00$ \\
\hline $\ln -118 m$ & $3.09 \mathrm{E}-03$ & $0.00 \mathrm{E}+00$ & - & - & - & - & $0.00 \mathrm{E}+00$ \\
\hline $\ln -120$ & $5.14 \mathrm{E}-04$ & $0.00 \mathrm{E}+00$ & - & - & - & - & $0.00 \mathrm{E}+00$ \\
\hline $\ln -120 m$ & $3.57 \mathrm{E}-05$ & - & - & - & - & - & $0.00 \mathrm{E}+00$ \\
\hline $\ln -123$ & $6.91 \mathrm{E}-05$ & $4.37 \mathrm{E}-13$ & 100 & 0 & 0 & 0 & $3.58 \mathrm{E}-09$ \\
\hline Sn-113 & $1.15 \mathrm{E}+02$ & $1.15 \mathrm{E}-06$ & 4.6 & 74.5 & 21.2 & 0 & $7.33 \mathrm{E}-02$ \\
\hline Sn-117m & $1.36 \mathrm{E}+01$ & $1.14 \mathrm{E}-07$ & 17.5 & 64.6 & 16.8 & 0.7 & $7.15 \mathrm{E}-03$ \\
\hline Sn-119m & $2.93 \mathrm{E}+02$ & $5.78 \mathrm{E}-07$ & 5.4 & 93.1 & 1.5 & 0 & $3.66 \mathrm{E}-02$ \\
\hline Sn-121 & $1.12 \mathrm{E}+00$ & $2.80 \mathrm{E}-09$ & 98.6 & 1.4 & 0 & 0 & $1.62 \mathrm{E}-04$ \\
\hline Sn-121m & $1.83 E+04$ & $8.01 \mathrm{E}-07$ & 6.5 & 93.4 & 0 & 0 & $5.08 \mathrm{E}-02$ \\
\hline Sn-123 & $1.29 \mathrm{E}+02$ & $2.78 \mathrm{E}-06$ & 6.4 & 93.2 & 0.2 & 0 & 1.76E-01 \\
\hline Sn-123m & $2.79 \mathrm{E}-02$ & $1.76 \mathrm{E}-10$ & 100 & 0 & 0 & 0 & 1.44E-06 \\
\hline Sn-125 & $9.64 \mathrm{E}+00$ & $3.01 \mathrm{E}-07$ & 27.4 & 61.8 & 10.2 & 0.5 & 1.89E-02 \\
\hline Sn-125m & $6.61 \mathrm{E}-03$ & $6.00 \mathrm{E}-12$ & 3.1 & 6.5 & 90.3 & 0.1 & $4.40 \mathrm{E}-07$ \\
\hline Sn-126 & $3.65 \mathrm{E}+07$ & $1.46 \mathrm{E}-05$ & 3 & 67.6 & 29.5 & 0 & 9.28E-01 \\
\hline Sn-127 & $8.75 \mathrm{E}-02$ & $2.09 \mathrm{E}-09$ & 74.2 & 2 & 23.8 & 0.2 & 1.18E-04 \\
\hline Sn-127m & 2.87E-03 & $4.73 \mathrm{E}-11$ & 39.5 & 21.8 & 34.5 & 4.2 & 3.56E-06 \\
\hline Sn-128 & $4.10 \mathrm{E}-02$ & $7.70 \mathrm{E}-10$ & 100 & 0 & 0 & 0 & 9.92E-06 \\
\hline Sn-130 & $2.58 \mathrm{E}-03$ & $0.00 \mathrm{E}+00$ & - & - & - & - & $0.00 \mathrm{E}+00$ \\
\hline Sn-132 & 4.63E-04 & $1.85 \mathrm{E}-10$ & 3 & 1.5 & 95.1 & 0.1 & $1.23 \mathrm{E}-05$ \\
\hline Sb-117 & 1.17E-01 & 1.13E-09 & 8.5 & 0 & 16.2 & 75 & 3.30E-05 \\
\hline Sb-120b & $5.76 \mathrm{E}+00$ & $3.99 \mathrm{E}-08$ & 51.6 & 48.4 & 0 & 0 & $2.49 \mathrm{E}-03$ \\
\hline Sb-122 & $2.70 \mathrm{E}+00$ & 5.06E-08 & 54.5 & 20.8 & 20 & 4.5 & 3.09E-03 \\
\hline Sb-124 & $6.02 \mathrm{E}+01$ & 1.32E-06 & 9.4 & 24.2 & 65.7 & 0.8 & $8.37 \mathrm{E}-02$ \\
\hline Sb-125 & $1.01 \mathrm{E}+03$ & 1.09E-06 & 5.3 & 18 & 76.1 & 0.2 & $6.87 \mathrm{E}-02$ \\
\hline Sb-126 & $1.24 \mathrm{E}+01$ & 4.31E-07 & 13.7 & 16.1 & 66.8 & 3.3 & 2.71E-02 \\
\hline Sb-126m & $1.32 \mathrm{E}-02$ & 6.06E-09 & 1.8 & 0.1 & 2.8 & 95.4 & $2.39 \mathrm{E}-05$ \\
\hline Sb-127 & $3.85 \mathrm{E}+00$ & 7.41E-08 & 42.9 & 23.1 & 29.4 & 4.6 & $4.77 \mathrm{E}-03$ \\
\hline Sb-128 & $3.75 \mathrm{E}-01$ & 9.32E-09 & 100 & 0 & 0 & 0 & $4.51 \mathrm{E}-04$ \\
\hline Sb-128m & 7.22E-03 & $3.81 \mathrm{E}-11$ & 100 & 0 & 0 & 0 & $4.34 \mathrm{E}-08$ \\
\hline Sb-129 & $1.80 \mathrm{E}-01$ & 1.29E-08 & 25.7 & 1.4 & 16 & 57.2 & $5.45 \mathrm{E}-04$ \\
\hline Sb-130 & $2.78 \mathrm{E}-02$ & $3.11 \mathrm{E}-10$ & 100 & 0 & 0 & 0 & $2.54 \mathrm{E}-06$ \\
\hline Sb-130m & $4.38 \mathrm{E}-03$ & $0.00 \mathrm{E}+00$ & - & - & - & - & $0.00 \mathrm{E}+00$ \\
\hline Sb-131 & $1.60 \mathrm{E}-02$ & $4.98 \mathrm{E}-09$ & 20.5 & 65.9 & 5.7 & 8 & $4.07 \mathrm{E}-03$ \\
\hline Sb-132 & $1.94 \mathrm{E}-03$ & $7.78 \mathrm{E}-10$ & 3.1 & 1.6 & 95.2 & 0.1 & $5.18 \mathrm{E}-05$ \\
\hline
\end{tabular}


Table 5. Effective Dose for Annual Average Releases (1 TBq/a) of fusion activation products (continued)

\begin{tabular}{|c|c|c|c|c|c|c|c|}
\hline Nuclide & $\begin{array}{c}\text { Nuclide } \\
\text { Half-life } \\
\text { (days) }\end{array}$ & $\begin{array}{l}\text { MEI 2-km } \\
\text { total EDE } \\
\text { (Sv/year) }\end{array}$ & $\begin{array}{l}\text { Inhalation } \\
\% \text { of } \\
\text { total dose }\end{array}$ & $\begin{array}{l}\text { Ingestion } \\
\% \text { of } \\
\text { total dose }\end{array}$ & $\begin{array}{c}\text { Ground } \\
\text { surfaces } \\
\% \text { of } \\
\text { total dose }\end{array}$ & $\begin{array}{c}\text { Air } \\
\text { immersion } \\
\% \text { of } \\
\text { total dose }\end{array}$ & $\begin{array}{c}2-100 \mathrm{~km} \\
\text { population } \\
\text { dose } \\
\text { (person- } \\
\text { Sv/year) }\end{array}$ \\
\hline Sb-132m & $2.92 \mathrm{E}-03$ & 1.16E-09 & 2.7 & 1.4 & 95.7 & 0.1 & 7.77E-05 \\
\hline Sb-133 & 1.67E-03 & $1.05 \mathrm{E}-09$ & 7.7 & 4.3 & 30.4 & 57.9 & $2.38 \mathrm{E}-05$ \\
\hline Sb-134 & $1.27 \mathrm{E}-04$ & $8.04 \mathrm{E}-11$ & 3.1 & 0 & 68.2 & 28.9 & $1.74 \mathrm{E}-06$ \\
\hline Te-121 & $1.70 \mathrm{E}+01$ & $1.18 \mathrm{E}-07$ & 8 & 20 & 69.7 & 2.4 & $7.44 \mathrm{E}-03$ \\
\hline Te-121m & $1.54 \mathrm{E}+02$ & $1.58 \mathrm{E}-06$ & 4.5 & 48.2 & 47.6 & 0.1 & $1.01 \mathrm{E}-01$ \\
\hline Te-123 & $3.65 \mathrm{E}+15$ & $7.19 \mathrm{E}-07$ & 3.8 & 94.2 & 2.1 & 0 & $4.56 \mathrm{E}-02$ \\
\hline Te-123m & $1.20 \mathrm{E}+02$ & $7.06 \mathrm{E}-07$ & 7.9 & 72 & 20.1 & 0.1 & $4.49 \mathrm{E}-02$ \\
\hline Te-125m & $5.80 \mathrm{E}+01$ & $2.63 \mathrm{E}-07$ & 15 & 81.4 & 3.8 & 0 & 1.67E-02 \\
\hline Te-127 & $3.90 \mathrm{E}-01$ & $1.73 \mathrm{E}-09$ & 97.7 & 0 & 0.9 & 1.4 & $8.46 \mathrm{E}-05$ \\
\hline Te-127m & $1.09 \mathrm{E}+02$ & $8.79 \mathrm{E}-07$ & 12.7 & 86.1 & 1.1 & 0 & $5.56 \mathrm{E}-02$ \\
\hline Te-129 & $4.83 \mathrm{E}-02$ & $6.31 \mathrm{E}-10$ & 57.1 & 0 & 3.3 & 39.6 & $9.04 \mathrm{E}-06$ \\
\hline Te-129m & $3.36 \mathrm{E}+01$ & $5.01 \mathrm{E}-07$ & 23.6 & 72.5 & 4.1 & 0 & $3.18 \mathrm{E}-02$ \\
\hline Te-131 & $1.74 \mathrm{E}-02$ & $2.97 \mathrm{E}-08$ & 6.9 & 83.5 & 4.1 & 5.7 & $5.10 \mathrm{E}-03$ \\
\hline Te-131m & $1.25 \mathrm{E}+00$ & $1.14 \mathrm{E}-07$ & 28.5 & 34.8 & 30.2 & 6.6 & $4.31 \mathrm{E}-02$ \\
\hline Te-132 & $3.26 \mathrm{E}+00$ & $1.31 \mathrm{E}-06$ & 3.5 & 1.9 & 94.7 & 0.1 & $8.68 \mathrm{E}-02$ \\
\hline Te-133 & $8.65 \mathrm{E}-03$ & $5.35 \mathrm{E}-09$ & 8 & 6.5 & 31.2 & 54.2 & $1.24 \mathrm{E}-04$ \\
\hline Te-133m & $3.85 \mathrm{E}-02$ & $1.46 \mathrm{E}-08$ & 13.8 & 0.1 & 12.8 & 73.3 & $2.30 \mathrm{E}-04$ \\
\hline Te-134 & $2.90 \mathrm{E}-02$ & $1.83 \mathrm{E}-08$ & 3.1 & 0 & 68.3 & 29 & $3.95 \mathrm{E}-04$ \\
\hline Te-135 & $2.22 \mathrm{E}-04$ & $6.44 \mathrm{E}-11$ & 8 & 0.2 & 81.4 & 10.5 & $2.87 \mathrm{E}-06$ \\
\hline $\mid-122$ & $2.51 \mathrm{E}-03$ & $9.06 \mathrm{E}-10$ & 0 & 0 & 7.8 & 92.3 & $1.68 \mathrm{E}-08$ \\
\hline $\mid-123$ & $5.50 \mathrm{E}-01$ & $2.01 \mathrm{E}-08$ & 7.9 & 5.4 & 82.6 & 4 & $1.05 \mathrm{E}-03$ \\
\hline $\mid-124$ & $4.18 \mathrm{E}+00$ & $1.35 \mathrm{E}-05$ & 0.8 & 93.3 & 5.3 & 0 & $8.32 \mathrm{E}-01$ \\
\hline $\mid-125$ & $6.01 \mathrm{E}+01$ & $1.21 \mathrm{E}-04$ & 0.1 & 99.2 & 0.2 & 0 & $7.64 \mathrm{E}+00$ \\
\hline I-126 & $1.30 \mathrm{E}+01$ & 7.54E-05 & 0.3 & 98.3 & 1.4 & 0 & $4.74 \mathrm{E}+00$ \\
\hline $\mid-128$ & $1.74 \mathrm{E}-02$ & $6.73 \mathrm{E}-10$ & 30.5 & 0 & 26 & 43.5 & $3.20 \mathrm{E}-06$ \\
\hline $\mid-129$ & $5.73 E+09$ & $2.07 \mathrm{E}-03$ & 0.1 & 100 & 0 & 0 & $1.31 \mathrm{E}+02$ \\
\hline $\mid-130$ & $5.15 \mathrm{E}-01$ & $2.17 \mathrm{E}-07$ & 6.7 & 3.5 & 84.8 & 5 & 1.13E-02 \\
\hline $\mathrm{I}-130 \mathrm{~m}$ & $6.25 \mathrm{E}-03$ & $2.04 \mathrm{E}-09$ & 3.7 & 1.9 & 91.7 & 2.8 & $1.15 \mathrm{E}-04$ \\
\hline $\mid-131$ & $8.04 \mathrm{E}+00$ & $3.95 \mathrm{E}-05$ & 0.5 & 98 & 1.4 & 0 & $2.47 \mathrm{E}+00$ \\
\hline $\mid-132$ & $9.58 \mathrm{E}-02$ & $4.79 \mathrm{E}-08$ & 3.9 & 0 & 72.4 & 23.8 & $1.22 \mathrm{E}-03$ \\
\hline $\mid-133$ & $8.67 \mathrm{E}-01$ & $2.09 \mathrm{E}-07$ & 15.2 & 41.2 & 42.2 & 1.5 & $1.18 \mathrm{E}-02$ \\
\hline $\mathrm{I}-133 \mathrm{~m}$ & $1.04 \mathrm{E}-04$ & $2.51 \mathrm{E}-11$ & 15.1 & 41.4 & 42.2 & 1.5 & $1.42 \mathrm{E}-06$ \\
\hline $\mid-134$ & 3.65E-02 & $2.66 \mathrm{E}-08$ & 2.2 & 0 & 51.9 & 45.9 & $2.90 \mathrm{E}-04$ \\
\hline $\mathrm{I}-134 \mathrm{~m}$ & $2.57 \mathrm{E}-03$ & 1.77E-09 & 1.9 & 0 & 57.1 & 41.1 & $2.15 \mathrm{E}-05$ \\
\hline $\mid-135$ & $2.75 \mathrm{E}-01$ & $7.98 \mathrm{E}-08$ & 8 & 0.2 & 81.2 & 10.5 & $3.55 \mathrm{E}-03$ \\
\hline I-136 & $9.61 \mathrm{E}-04$ & 1.39E-10 & 0 & 0 & 2.4 & 97.8 & $0.00 \mathrm{E}+00$ \\
\hline $\mid-138$ & 7.41E-05 & $4.60 \mathrm{E}-11$ & 1.2 & 0 & 2.8 & 96.1 & $3.86 \mathrm{E}-07$ \\
\hline $\mid-139$ & $2.78 \mathrm{E}-05$ & $4.17 \mathrm{E}-12$ & 4.8 & 0 & 1 & 94.2 & $1.30 \mathrm{E}-08$ \\
\hline $\mathrm{Xe}-122$ & 8.38E-01 & 1.42E-07 & 0 & 0 & 97.2 & 3.1 & 8.01E-03 \\
\hline Xe-123 & $8.67 \mathrm{E}-02$ & 5.71E-09 & 0.2 & 0.2 & 46.2 & 53.6 & $2.44 \mathrm{E}-04$ \\
\hline Xe-125 & 7.08E-01 & $2.05 \mathrm{E}-07$ & 0 & 98 & 1.4 & 0.6 & 1.30E-02 \\
\hline Xe-127 & $3.64 \mathrm{E}+01$ & 1.33E-09 & 0 & 0 & 0 & 100 & 8.42E-05 \\
\hline Xe-129m & $8.89 \mathrm{E}+00$ & 1.16E-10 & 0 & 0 & 0 & 100 & $7.28 \mathrm{E}-06$ \\
\hline Xe-131m & $1.18 \mathrm{E}+01$ & $4.29 \mathrm{E}-11$ & 0 & 0 & 0 & 100 & $2.70 \mathrm{E}-06$ \\
\hline
\end{tabular}


Table 5. Effective Dose for Annual Average Releases (1 TBq/a) of fusion activation products (continued)

\begin{tabular}{|c|c|c|c|c|c|c|c|}
\hline Nuclide & $\begin{array}{c}\text { Nuclide } \\
\text { Half-life } \\
\text { (days) }\end{array}$ & $\begin{array}{l}\text { MEl 2-km } \\
\text { total EDE } \\
\text { (Sv/year) }\end{array}$ & $\begin{array}{l}\text { Inhalation } \\
\% \text { of } \\
\text { total dose }\end{array}$ & $\begin{array}{l}\text { Ingestion } \\
\% \text { of } \\
\text { total dose } \\
\end{array}$ & $\begin{array}{c}\text { Ground } \\
\text { surfaces } \\
\% \text { of } \\
\text { total dose }\end{array}$ & $\begin{array}{c}\text { Air } \\
\text { immersion } \\
\% \text { of } \\
\text { total dose } \\
\end{array}$ & $\begin{array}{c}2-100 \mathrm{~km} \\
\text { population } \\
\text { dose } \\
\text { (person- } \\
\text { Sv/year) } \\
\end{array}$ \\
\hline Xe-133 & $5.25 \mathrm{E}+00$ & $1.76 \mathrm{E}-10$ & 0 & 0 & 0 & 100 & $1.09 \mathrm{E}-05$ \\
\hline Xe-133m & $2.19 \mathrm{E}+00$ & $1.49 \mathrm{E}-10$ & 0 & 0 & 0 & 100 & $9.24 \mathrm{E}-06$ \\
\hline Xe-135 & $3.79 \mathrm{E}-01$ & $1.25 \mathrm{E}-09$ & 0 & 0 & 0 & 100 & $6.05 \mathrm{E}-05$ \\
\hline Xe-135m & $1.07 \mathrm{E}-02$ & $1.43 \mathrm{E}-09$ & 0 & 0 & 0 & 100 & $5.10 \mathrm{E}-06$ \\
\hline Xe-137 & $2.66 \mathrm{E}-03$ & $1.81 \mathrm{E}-10$ & 0 & 0.4 & 0.2 & 99.4 & $2.16 \mathrm{E}-07$ \\
\hline Xe-138 & $9.84 \mathrm{E}-03$ & $6.10 \mathrm{E}-09$ & 1.2 & 0 & 2.8 & 96.1 & $5.11 \mathrm{E}-05$ \\
\hline Xe-139 & $4.57 \mathrm{E}-04$ & $6.84 \mathrm{E}-11$ & 4.8 & 0 & 1 & 94.2 & $2.13 \mathrm{E}-07$ \\
\hline $\mathrm{Xe}-140$ & $1.57 \mathrm{E}-04$ & $4.14 \mathrm{E}-12$ & 6.3 & 20.2 & 73.2 & 0.3 & $2.68 \mathrm{E}-07$ \\
\hline Xe-141 & 1.99E-05 & $5.95 \mathrm{E}-12$ & 9.8 & 0 & 2.1 & 88.1 & $3.31 \mathrm{E}-08$ \\
\hline Cs-126 & $1.14 \mathrm{E}-03$ & $1.14 \mathrm{E}-10$ & 0 & 0 & 0.2 & 100 & $1.07 \mathrm{E}-12$ \\
\hline Cs-129 & $1.34 \mathrm{E}+00$ & 5.69E-09 & 15.5 & 4.7 & 56.8 & 23 & $3.33 \mathrm{E}-04$ \\
\hline Cs-131 & $9.69 \mathrm{E}+00$ & $5.77 \mathrm{E}-09$ & 16.3 & 55.6 & 27.4 & 0.6 & $3.62 \mathrm{E}-04$ \\
\hline Cs-132 & $6.48 \mathrm{E}+00$ & $6.64 \mathrm{E}-08$ & 10.6 & 25.3 & 58.6 & 5.4 & $4.15 \mathrm{E}-03$ \\
\hline Cs-134 & $7.53 \mathrm{E}+02$ & $1.89 \mathrm{E}-05$ & 1.5 & 84.1 & 14.8 & 0 & $1.20 \mathrm{E}+00$ \\
\hline Cs-134m & $1.21 \mathrm{E}-01$ & $1.23 \mathrm{E}-09$ & 16.7 & 36.5 & 38.8 & 8.1 & $7.03 \mathrm{E}-05$ \\
\hline Cs-135 & $8.40 \mathrm{E}+08$ & $1.81 \mathrm{E}-06$ & 1.5 & 98.9 & 0 & 0 & $1.15 \mathrm{E}-01$ \\
\hline Cs-135m & $3.68 \mathrm{E}-02$ & $1.36 \mathrm{E}-10$ & 100 & 0 & 0 & 0 & $1.49 \mathrm{E}-06$ \\
\hline Cs-136 & $1.31 \mathrm{E}+01$ & 4.87E-07 & 9.1 & 40.9 & 47.8 & 2.3 & $3.06 \mathrm{E}-02$ \\
\hline Cs-137 & $1.10 \mathrm{E}+04$ & $1.37 \mathrm{E}-05$ & 1.4 & 89.8 & 8.8 & 0 & $8.70 \mathrm{E}-01$ \\
\hline Cs-138 & $2.24 \mathrm{E}-02$ & $1.13 \mathrm{E}-08$ & 3.8 & 0 & 2.9 & 92.9 & $7.23 \mathrm{E}-05$ \\
\hline Cs-138m & $2.01 \mathrm{E}-03$ & $7.27 \mathrm{E}-10$ & 3.8 & 0 & 3.3 & 93 & $5.37 \mathrm{E}-06$ \\
\hline Cs-139 & $6.53 \mathrm{E}-03$ & $9.16 \mathrm{E}-10$ & 5.5 & 0 & 1 & 93.4 & $2.98 \mathrm{E}-06$ \\
\hline Cs-140 & 7.39E-04 & 1.94E-11 & 6.3 & 20.3 & 73.2 & 0.3 & $1.26 \mathrm{E}-06$ \\
\hline Cs-141 & $2.89 \mathrm{E}-04$ & $8.64 \mathrm{E}-11$ & 9.8 & 0 & 2.1 & 88.1 & $4.80 \mathrm{E}-07$ \\
\hline Cs-144 & $1.18 \mathrm{E}-05$ & $1.14 \mathrm{E}-13$ & 75.1 & 22.3 & 2.6 & 0 & $7.24 \mathrm{E}-09$ \\
\hline Ba-131 & $1.18 \mathrm{E}+01$ & $6.86 \mathrm{E}-08$ & 5.8 & 17.6 & 73.3 & 3.4 & $4.32 \mathrm{E}-03$ \\
\hline Ba-133 & $3.92 \mathrm{E}+03$ & 1.13E-06 & 3.6 & 21.4 & 75.1 & 0.2 & $7.20 \mathrm{E}-02$ \\
\hline Ba-133m & $1.62 \mathrm{E}+00$ & $6.92 \mathrm{E}-09$ & 47.5 & 29.8 & 18.5 & 4.1 & $4.13 \mathrm{E}-04$ \\
\hline Ba-135m & $1.20 \mathrm{E}+00$ & 4.51E-09 & 57.2 & 23.5 & 13.7 & 5.5 & 2.62E-04 \\
\hline Ba-137m & 1.77E-03 & $2.49 \mathrm{E}-10$ & 0 & 0 & 0.3 & 100 & $3.34 \mathrm{E}-10$ \\
\hline Ba-139 & $5.77 \mathrm{E}-02$ & 1.06E-09 & 82.4 & 0 & 1.6 & 15.8 & 1.79E-05 \\
\hline Ba-140 & $1.28 \mathrm{E}+01$ & 3.37E-07 & 6.3 & 20.3 & 73 & 0.3 & $2.17 E-02$ \\
\hline Ba-141 & 1.27E-02 & $3.71 \mathrm{E}-09$ & 9.9 & 0 & 2.1 & 87.9 & 2.08E-05 \\
\hline Ba-142 & 7.43E-03 & 3.68E-09 & 4.4 & 0 & 4.8 & 91 & 4.25E-05 \\
\hline Ba-144 & 1.27E-04 & $1.23 \mathrm{E}-12$ & 75 & 22.3 & 2.6 & 0 & 7.81E-08 \\
\hline La-137 & $2.19 \mathrm{E}+07$ & 1.43E-07 & 78.3 & 21.7 & 0 & 0 & 9.06E-03 \\
\hline La-138 & $4.93 \mathrm{E}+13$ & $2.61 \mathrm{E}-06$ & 83.5 & 16.3 & 0 & 0 & $1.65 \mathrm{E}-01$ \\
\hline La-140 & $1.68 \mathrm{E}+00$ & $7.55 \mathrm{E}-08$ & 34.3 & 10.5 & 38.9 & 16.3 & $4.50 \mathrm{E}-03$ \\
\hline La-141 & 1.64E-01 & 2.99E-09 & 86.6 & 0.6 & 5.7 & 7.3 & 1.22E-04 \\
\hline La-142 & $6.63 \mathrm{E}-02$ & 1.69E-08 & 5.2 & 0 & 7.4 & 87 & $3.22 \mathrm{E}-04$ \\
\hline La-143 & $9.72 \mathrm{E}-03$ & $2.81 \mathrm{E}-10$ & 90.4 & 0.2 & 8.1 & 1.2 & 1.03E-05 \\
\hline La-144 & 4.63E-04 & 4.47E-12 & 75.2 & 22.3 & 2.6 & 0 & 2.84E-07 \\
\hline Ce-139 & $1.38 \mathrm{E}+02$ & 2.43E-07 & 18.2 & 12.1 & 69.1 & 0.3 & $1.54 \mathrm{E}-02$ \\
\hline Ce-141 & $3.25 E+01$ & 1.10E-07 & 45.5 & 32.7 & 21.4 & 0.4 & $6.95 \mathrm{E}-03$ \\
\hline
\end{tabular}


Table 5. Effective Dose for Annual Average Releases (1 TBq/a) of fusion activation products (continued)

\begin{tabular}{|c|c|c|c|c|c|c|c|}
\hline Nuclide & $\begin{array}{c}\text { Nuclide } \\
\text { Half-life } \\
\text { (days) }\end{array}$ & $\begin{array}{l}\text { MEI 2-km } \\
\text { total EDE } \\
\text { (Sv/year) }\end{array}$ & $\begin{array}{l}\text { Inhalation } \\
\% \text { of } \\
\text { total dose }\end{array}$ & $\begin{array}{l}\text { Ingestion } \\
\% \text { of } \\
\text { total dose }\end{array}$ & $\begin{array}{c}\text { Ground } \\
\text { surfaces } \\
\% \text { of } \\
\text { total dose }\end{array}$ & $\begin{array}{c}\text { Air } \\
\text { immersion } \\
\% \text { of } \\
\text { total dose }\end{array}$ & $\begin{array}{c}2-100 \mathrm{~km} \\
\text { population } \\
\text { dose } \\
\text { (person- } \\
\text { Sv/year) }\end{array}$ \\
\hline Ce-143 & $1.38 \mathrm{E}+00$ & $2.35 \mathrm{E}-08$ & 80 & 0.4 & 13.7 & 5.6 & $1.42 \mathrm{E}-03$ \\
\hline $\mathrm{Ce}-144$ & $2.84 \mathrm{E}+02$ & $2.75 \mathrm{E}-06$ & 74.9 & 22.3 & 2.6 & 0 & $1.74 \mathrm{E}-01$ \\
\hline Ce-145 & $2.08 \mathrm{E}-03$ & $2.75 \mathrm{E}-11$ & 100 & 0 & 0 & 0 & 1.33E-06 \\
\hline Ce-146 & $9.86 \mathrm{E}-03$ & $0.00 \mathrm{E}+00$ & - & - & - & - & $0.00 \mathrm{E}+00$ \\
\hline Pr-142 & $7.97 \mathrm{E}-01$ & 1.81E-08 & 87.3 & 9.3 & 1.9 & 1.7 & $1.01 \mathrm{E}-03$ \\
\hline Pr-143 & $1.36 \mathrm{E}+01$ & 7.99E-08 & 53.8 & 46.2 & 0 & 0 & $5.03 \mathrm{E}-03$ \\
\hline Pr-144 & $1.20 \mathrm{E}-02$ & $2.97 \mathrm{E}-10$ & 57.6 & 0 & 0.7 & 41.8 & $8.54 \mathrm{E}-07$ \\
\hline Pr-144m & $5.00 \mathrm{E}-03$ & $9.74 \mathrm{E}-11$ & 50.7 & 0 & 1.3 & 47.9 & $5.61 \mathrm{E}-07$ \\
\hline Pr-145 & $2.49 \mathrm{E}-01$ & $3.70 \mathrm{E}-09$ & 100 & 0 & 0 & 0 & $1.58 \mathrm{E}-04$ \\
\hline Pr-146 & $1.68 \mathrm{E}-02$ & $0.00 \mathrm{E}+00$ & - & - & - & - & $0.00 \mathrm{E}+00$ \\
\hline Pr-147 & $8.33 \mathrm{E}-03$ & $1.25 \mathrm{E}-10$ & 84 & 7.6 & 8.2 & 0.2 & $3.77 \mathrm{E}-06$ \\
\hline Pr-148 & $1.60 \mathrm{E}-03$ & $0.00 \mathrm{E}+00$ & - & - & - & - & $0.00 \mathrm{E}+00$ \\
\hline Pr-149 & $1.60 \mathrm{E}-03$ & $6.75 \mathrm{E}-11$ & 35.4 & 0 & 7.9 & 56.6 & $1.97 \mathrm{E}-06$ \\
\hline $\mathrm{Nd}-141$ & $1.04 \mathrm{E}-01$ & $4.63 \mathrm{E}-11$ & 100 & 0 & 0 & 0 & $1.25 \mathrm{E}-06$ \\
\hline $\mathrm{Nd}-147$ & $1.10 \mathrm{E}+01$ & $7.68 \mathrm{E}-08$ & 47.5 & 34 & 17.6 & 0.9 & $4.83 \mathrm{E}-03$ \\
\hline $\mathrm{Nd}-149$ & $7.21 \mathrm{E}-02$ & $3.19 \mathrm{E}-09$ & 35.7 & 0.4 & 7.4 & 56.4 & $9.51 \mathrm{E}-05$ \\
\hline $\mathrm{Nd}-151$ & $8.61 \mathrm{E}-03$ & $1.49 \mathrm{E}-10$ & 79.9 & 0 & \begin{tabular}{ll|}
17.1 \\
\end{tabular} & 3.3 & $6.28 \mathrm{E}-06$ \\
\hline$P m-143$ & $2.65 \mathrm{E}+02$ & $5.45 \mathrm{E}-07$ & 7.7 & 14.2 & 77.8 & 0.3 & $3.45 \mathrm{E}-02$ \\
\hline $\mathrm{Pm}-144$ & $3.63 E+02$ & $2.98 \mathrm{E}-06$ & 8.1 & 11.2 & 80.2 & 0.3 & $1.89 \mathrm{E}-01$ \\
\hline$P m-145$ & $6.46 \mathrm{E}+03$ & $2.64 \mathrm{E}-07$ & 60.2 & 17.3 & 22.3 & 0 & 1.67E-02 \\
\hline$P m-146$ & $2.02 \mathrm{E}+03$ & $2.48 \mathrm{E}-06$ & 26.1 & 12.6 & 61.3 & 0.2 & $1.57 \mathrm{E}-01$ \\
\hline $\mathrm{Pm}-147$ & $9.57 \mathrm{E}+02$ & $2.90 \mathrm{E}-07$ & 69 & 30.9 & 0 & 0 & 1.84E-02 \\
\hline $\mathrm{Pm}-148$ & $5.37 \mathrm{E}+00$ & $1.21 \mathrm{E}-07$ & 48.7 & 29.1 & 19.8 & 2.5 & 7.52E-03 \\
\hline $\mathrm{Pm}-148 \mathrm{~m}$ & $4.13 E+01$ & 1.04E-06 & 9.1 & 22 & 67.6 & 1 & 6.56E-02 \\
\hline$P m-149$ & $2.21 \mathrm{E}+00$ & $2.20 \mathrm{E}-08$ & 75 & 23.9 & 1 & 0.3 & 1.33E-03 \\
\hline $\mathrm{Pm}-150$ & 1.12E-01 & 1.64E-09 & 100 & 0 & 0 & 0 & 4.67E-05 \\
\hline $\mathrm{Pm}-151$ & $1.18 \mathrm{E}+00$ & $1.46 \mathrm{E}-08$ & 64.3 & 0 & 24 & 11.5 & 8.46E-04 \\
\hline Pm-152 & $2.85 E-03$ & $0.00 \mathrm{E}+00$ & - & - & - & - & $0.00 \mathrm{E}+00$ \\
\hline $\mathrm{Pm}-153$ & $3.75 \mathrm{E}-03$ & $1.59 \mathrm{E}-11$ & 84.3 & 0.1 & 13.6 & 2.2 & $1.32 \mathrm{E}-06$ \\
\hline $\mathrm{Pm}-154$ & 1.94E-03 & $0.00 \mathrm{E}+00$ & - & - & - & - & $0.00 \mathrm{E}+00$ \\
\hline Sm-146 & $3.76 \mathrm{E}+10$ & $4.80 \mathrm{E}-04$ & 95.8 & 4.2 & 0 & 0 & $3.04 \mathrm{E}+01$ \\
\hline Sm-147 & $3.91 \mathrm{E}+13$ & $4.36 \mathrm{E}-04$ & 95.9 & 4.2 & 0 & 0 & $2.77 \mathrm{E}+01$ \\
\hline Sm-151 & $3.29 \mathrm{E}+04$ & $2.05 \mathrm{E}-07$ & 83.4 & 16.7 & 0 & 0 & 1.30E-02 \\
\hline Sm-153 & $1.95 E+00$ & $1.46 \mathrm{E}-08$ & 68.4 & 22.3 & 7.7 & 1.8 & 8.78E-04 \\
\hline Sm-155 & 1.54E-02 & $1.08 \mathrm{E}-10$ & 98.1 & 0.2 & 1.1 & 0 & 6.77E-07 \\
\hline Sm-156 & 3.92E-01 & 3.39E-09 & 97.9 & 2 & 0.1 & 0 & 1.66E-04 \\
\hline Eu-150b & $1.25 E+04$ & $2.21 \mathrm{E}-06$ & 71.9 & 28.1 & 0 & 0 & 1.40E-01 \\
\hline Eu-152 & $4.96 \mathrm{E}+03$ & 4.17E-06 & 31.2 & 14.3 & 54.7 & 0.1 & $2.65 \mathrm{E}-01$ \\
\hline Eu-152m & 3.88E-01 & 7.17E-09 & 61.8 & 1.7 & 13.9 & 22.6 & 3.50E-04 \\
\hline Eu-154 & $3.14 \mathrm{E}+03$ & 4.86E-06 & 31.5 & 18.4 & 50 & 0.1 & $3.08 \mathrm{E}-01$ \\
\hline Eu-155 & $1.81 \mathrm{E}+03$ & $4.95 \mathrm{E}-07$ & 46.5 & 25.5 & 28.1 & 0.1 & 3.14E-02 \\
\hline Eu-156 & $1.52 \mathrm{E}+01$ & $3.22 \mathrm{E}-07$ & 20.1 & 28.7 & 49.1 & 2.3 & 2.03E-02 \\
\hline Eu-157 & 6.33E-01 & 6.34E-09 & 92.3 & 7.6 & 0 & 0 & 3.41E-04 \\
\hline Eu-158 & $3.19 \mathrm{E}-02$ & $4.30 \mathrm{E}-10$ & 100 & 0 & 0 & 0 & $4.06 \mathrm{E}-06$ \\
\hline
\end{tabular}


Table 5. Effective Dose for Annual Average Releases (1 TBq/a) of fusion activation products (continued)

\begin{tabular}{|c|c|c|c|c|c|c|c|}
\hline Nuclide & $\begin{array}{c}\text { Nuclide } \\
\text { Half-life } \\
\text { (days) }\end{array}$ & $\begin{array}{l}\text { MEI 2-km } \\
\text { total EDE } \\
\text { (Sv/year) }\end{array}$ & $\begin{array}{l}\text { Inhalation } \\
\% \text { of } \\
\text { total dose }\end{array}$ & $\begin{array}{l}\text { Ingestion } \\
\% \% \text { of } \\
\text { total dose }\end{array}$ & $\begin{array}{c}\text { Ground } \\
\text { surfaces } \\
\% \text { of } \\
\text { total dose } \\
\end{array}$ & $\begin{array}{c}\text { Air } \\
\text { immersion } \\
\% \text { of } \\
\text { total dose }\end{array}$ & $\begin{array}{c}2-100 \mathrm{~km} \\
\text { population } \\
\text { dose } \\
\text { (person- } \\
\text { Sv/year) } \\
\end{array}$ \\
\hline Eu-159 & $1.26 \mathrm{E}-02$ & $3.08 \mathrm{E}-11$ & 82.5 & 0 & 14.6 & 3 & $5.01 \mathrm{E}-06$ \\
\hline Gd-148 & $3.40 \mathrm{E}+04$ & $5.14 \mathrm{E}-04$ & 96.3 & 3.7 & 0 & 0 & $3.26 \mathrm{E}+01$ \\
\hline Gd-152 & $3.94 \mathrm{E}+16$ & $3.73 \mathrm{E}-04$ & 96.2 & 3.7 & 0 & 0 & $2.37 \mathrm{E}+01$ \\
\hline Gd-153 & $2.42 \mathrm{E}+02$ & $2.93 \mathrm{E}-07$ & 16.9 & 27.4 & 55.6 & 0.2 & $1.86 \mathrm{E}-02$ \\
\hline Gd-159 & $7.73 \mathrm{E}-01$ & $5.68 \mathrm{E}-09$ & 91.7 & 0 & 4.8 & 3.3 & $3.15 \mathrm{E}-04$ \\
\hline Tb-157 & $5.48 \mathrm{E}+04$ & $7.25 \mathrm{E}-08$ & 73.1 & 13.5 & 13.3 & 0 & $4.60 \mathrm{E}-03$ \\
\hline Tb-158 & $5.48 \mathrm{E}+04$ & 1.86E-06 & 79 & 21 & 0 & 0 & $1.18 \mathrm{E}-01$ \\
\hline Tb-160 & $7.23 \mathrm{E}+01$ & 1.06E-06 & 12.3 & 29.5 & 57.7 & 0.5 & $6.71 \mathrm{E}-02$ \\
\hline Tb-161 & $6.91 \mathrm{E}+00$ & $3.04 \mathrm{E}-08$ & 60.2 & 40.1 & 0 & 0 & $1.90 \mathrm{E}-03$ \\
\hline Dy-157 & $3.38 \mathrm{E}-01$ & $3.18 \mathrm{E}-09$ & 13.9 & 0.4 & 32.7 & 52.8 & $1.50 \mathrm{E}-04$ \\
\hline Dy-159 & $1.44 \mathrm{E}+02$ & 4.09E-08 & 30.3 & 69.7 & 0 & 0 & 2.59E-03 \\
\hline Dy-165 & $9.73 \mathrm{E}-02$ & $7.59 \mathrm{E}-10$ & 81.6 & 0 & 2.8 & 15.7 & 1.96E-05 \\
\hline Dy-166 & $3.40 \mathrm{E}+00$ & $5.75 \mathrm{E}-08$ & 70.8 & 25.6 & 3.5 & 0.3 & $3.72 \mathrm{E}-03$ \\
\hline Ho-164 & $2.01 \mathrm{E}-02$ & $3.78 \mathrm{E}-11$ & 100 & 0 & 0 & 0 & $2.15 \mathrm{E}-07$ \\
\hline Ho-164m & $2.60 \mathrm{E}-02$ & $9.30 \mathrm{E}-11$ & 100 & 0 & 0 & 0 & $1.03 \mathrm{E}-06$ \\
\hline Ho-166 & $1.12 \mathrm{E}+00$ & $1.68 \mathrm{E}-08$ & 97.6 & 0 & 1.6 & 0.8 & $9.72 \mathrm{E}-04$ \\
\hline Ho-166m & $4.38 \mathrm{E}+05$ & 8.46E-06 & 50.1 & 9 & 40.8 & 0.1 & 5.37E-01 \\
\hline Er-169 & $9.30 \mathrm{E}+00$ & 2.04E-08 & 57.8 & 42.2 & 0 & 0 & $1.28 \mathrm{E}-03$ \\
\hline Er-171 & 3.13E-01 & 5.83E-09 & 49.7 & 0.9 & 17.8 & 31.4 & 2.69E-04 \\
\hline $\mathrm{Tm}-170$ & $1.29 \mathrm{E}+02$ & $4.65 \mathrm{E}-07$ & 29.2 & 69.7 & 1.2 & 0 & 2.95E-02 \\
\hline $\mathrm{Tm}-171$ & $7.01 \mathrm{E}+02$ & 8.69E-08 & 58.3 & 40.2 & 1.6 & 0 & 5.51E-03 \\
\hline Yb-169 & $3.20 \mathrm{E}+01$ & $2.04 \mathrm{E}-07$ & 20.2 & 33.3 & 46 & 0.7 & 1.29E-02 \\
\hline Yb-175 & $4.19 \mathrm{E}+00$ & $1.51 \mathrm{E}-08$ & 58.5 & 30.7 & 9.7 & 1.3 & 9.37E-04 \\
\hline Lu-174 & $1.21 \mathrm{E}+03$ & $2.80 \mathrm{E}-07$ & 67.5 & 32.7 & 0 & 0 & 1.78E-02 \\
\hline Lu-174m & $1.42 \mathrm{E}+02$ & $2.57 \mathrm{E}-07$ & 52.9 & 47.5 & 0 & 0 & 1.63E-02 \\
\hline Lu-176 & $1.31 \mathrm{E}+13$ & $4.36 \mathrm{E}-06$ & 85.1 & 14.8 & 0 & 0 & $2.77 \mathrm{E}-01$ \\
\hline Lu-176m & $1.53 \mathrm{E}-01$ & 1.26E-09 & 100 & 0.1 & 0 & 0 & 4.33E-05 \\
\hline Lu-177 & $2.80 \mathrm{E}-01$ & $1.36 \mathrm{E}-08$ & 97.8 & 0.3 & 0.7 & 1.3 & 6.06E-04 \\
\hline Lu-177 & $2.80 \mathrm{E}-01$ & 1.36E-08 & 97.8 & 0.3 & 0.7 & 1.3 & 6.06E-04 \\
\hline Lu-177m & $6.70 \mathrm{E}+00$ & 4.62E-07 & 79 & 6.7 & 13.1 & 1.1 & $2.88 \mathrm{E}-02$ \\
\hline Lu-178 & 1.97E-02 & $2.07 \mathrm{E}-10$ & 100 & 0 & 0 & 0 & 1.15E-06 \\
\hline Lu-178m & $1.58 \mathrm{E}-02$ & $1.29 \mathrm{E}-10$ & 100 & 0 & 0 & 0 & 5.42E-07 \\
\hline Hf-175 & $7.00 \mathrm{E}+01$ & $9.02 \mathrm{E}-08$ & 29.4 & 70.6 & 0 & 0 & 5.71E-03 \\
\hline $\mathrm{Hf}-177 \mathrm{~m}$ & $3.57 \mathrm{E}-02$ & $3.02 \mathrm{E}-10$ & 100 & 0 & 0 & 0 & 3.20E-06 \\
\hline $\mathrm{Hf}-178 \mathrm{~m}$ & $1.13 \mathrm{E}+04$ & $4.79 \mathrm{E}-06$ & 68.9 & 31.1 & 0 & 0 & 3.04E-01 \\
\hline $\mathrm{Hf}-179 \mathrm{~m}$ & $2.51 \mathrm{E}+01$ & $1.29 \mathrm{E}-07$ & 37.9 & 61.9 & 0 & 0 & $8.13 \mathrm{E}-03$ \\
\hline Hf-181 & $4.24 \mathrm{E}+01$ & $3.89 \mathrm{E}-07$ & 16.7 & 30.8 & 51.9 & 0.7 & $2.46 \mathrm{E}-02$ \\
\hline Hf-182 & $3.29 \mathrm{E}+09$ & $5.23 \mathrm{E}-06$ & 79.9 & 20.1 & 0 & 0 & $3.32 \mathrm{E}-01$ \\
\hline Hf-183 & 4.44E-02 & $5.25 \mathrm{E}-10$ & 96.8 & 3.2 & 0 & 0 & $2.59 \mathrm{E}-05$ \\
\hline Ta-179 & $6.65 \mathrm{E}+02$ & $5.11 \mathrm{E}-08$ & 66.9 & 33.1 & 0 & 0 & $3.24 \mathrm{E}-03$ \\
\hline Ta-180m & $3.38 \mathrm{E}-01$ & $5.03 \mathrm{E}-10$ & 98.2 & 1.8 & 0 & 0 & $2.37 \mathrm{E}-05$ \\
\hline Ta-182 & $1.15 \mathrm{E}+02$ & 1.55E-06 & 14.1 & 19 & 66.5 & 0.4 & $9.83 \mathrm{E}-02$ \\
\hline Ta-182m & $1.10 \mathrm{E}-02$ & $4.71 \mathrm{E}-11$ & 100 & 0 & 0 & 0 & $1.19 \mathrm{E}-07$ \\
\hline Ta-183 & $5.10 \mathrm{E}+00$ & $4.40 \mathrm{E}-08$ & 64.3 & 35.7 & 0 & 0 & 2.73E-03 \\
\hline
\end{tabular}


Table 5. Effective Dose for Annual Average Releases (1 TBq/a) of fusion activation products (continued)

\begin{tabular}{|c|c|c|c|c|c|c|c|}
\hline Nuclide & $\begin{array}{c}\text { Nuclide } \\
\text { Half-life } \\
\text { (days) }\end{array}$ & $\begin{array}{l}\text { MEI 2-km } \\
\text { total EDE } \\
\text { (Sv/year) }\end{array}$ & $\begin{array}{l}\text { Inhalation } \\
\% \text { of } \\
\text { total dose }\end{array}$ & $\begin{array}{l}\text { Ingestion } \\
\% \text { of } \\
\text { total dose }\end{array}$ & $\begin{array}{c}\text { Ground } \\
\text { surfaces } \\
\% \text { of } \\
\text { total dose }\end{array}$ & $\begin{array}{c}\text { Air } \\
\text { immersion } \\
\% \text { of } \\
\text { total dose }\end{array}$ & $\begin{array}{c}2-100 \mathrm{~km} \\
\text { population } \\
\text { dose } \\
\text { (person- } \\
\text { Sv/year) } \\
\end{array}$ \\
\hline Ta-184 & $3.63 \mathrm{E}-01$ & $6.55 \mathrm{E}-09$ & 97.7 & 2.2 & 0 & 0 & $3.14 \mathrm{E}-04$ \\
\hline Ta-185 & $3.40 \mathrm{E}-02$ & $4.07 \mathrm{E}-10$ & 95.3 & 4.7 & 0 & 0 & $1.08 \mathrm{E}-05$ \\
\hline Ta-186 & $7.29 \mathrm{E}-03$ & $7.35 \mathrm{E}-11$ & 100 & 0 & 0 & 0 & $8.51 \mathrm{E}-08$ \\
\hline W-179 & $2.60 \mathrm{E}-02$ & $1.53 \mathrm{E}-11$ & 98.7 & 1 & 0 & 0 & $2.21 \mathrm{E}-07$ \\
\hline W-181 & $1.21 \mathrm{E}+02$ & $1.05 \mathrm{E}-07$ & 0.8 & 59.9 & 38.7 & 0.2 & $6.62 \mathrm{E}-03$ \\
\hline W-185 & $7.51 \mathrm{E}+01$ & $2.90 \mathrm{E}-07$ & 1.5 & 98.3 & 0 & 0 & $1.83 \mathrm{E}-02$ \\
\hline W-187 & $9.96 \mathrm{E}-01$ & 1.09E-08 & 28.5 & 11.7 & 37.2 & 22.2 & $6.21 \mathrm{E}-04$ \\
\hline W-188 & $6.94 \mathrm{E}+01$ & $1.35 \mathrm{E}-06$ & 1.8 & 95.6 & 2.6 & 0 & $8.54 \mathrm{E}-02$ \\
\hline $\operatorname{Re}-182 a$ & $5.29 \mathrm{E}-01$ & $7.01 \mathrm{E}-09$ & 27.5 & 1.6 & 70.9 & 0 & $3.66 \mathrm{E}-04$ \\
\hline $\operatorname{Re}-182 b$ & $2.67 \mathrm{E}+00$ & $6.78 \mathrm{E}-08$ & 19.9 & 10.6 & 56 & 13.2 & $4.13 \mathrm{E}-03$ \\
\hline Re-184 & $3.80 \mathrm{E}+01$ & $3.94 \mathrm{E}-07$ & 5.4 & 21.1 & 72.1 & 1.2 & $2.49 \mathrm{E}-02$ \\
\hline $\mathrm{Re}-184 \mathrm{~m}$ & $1.65 \mathrm{E}+02$ & $1.44 \mathrm{E}-06$ & 4.9 & 18.5 & 76.4 & 0.1 & $9.14 \mathrm{E}-02$ \\
\hline Re-186 & $3.78 \mathrm{E}+00$ & $2.66 \mathrm{E}-08$ & 66.5 & 30.5 & 2.8 & 0.4 & $1.64 \mathrm{E}-03$ \\
\hline Re-186m & $7.30 \mathrm{E}+07$ & $1.44 \mathrm{E}-06$ & 13.5 & 83.3 & 3.4 & 0 & $9.14 \mathrm{E}-02$ \\
\hline Re-187 & $1.83 \mathrm{E}+13$ & $3.08 \mathrm{E}-09$ & 9.4 & 90.6 & 0 & 0 & 1.96E-04 \\
\hline Re-188 & $7.08 \mathrm{E}-01$ & $1.13 \mathrm{E}-08$ & 92.9 & 0.5 & 3.2 & 2.6 & $6.16 \mathrm{E}-04$ \\
\hline Re-188m & $1.29 \mathrm{E}-02$ & $2.19 \mathrm{E}-10$ & 96.3 & 0.1 & 3 & 0.7 & $1.15 \mathrm{E}-05$ \\
\hline Re-189 & $1.01 \mathrm{E}+00$ & $7.28 \mathrm{E}-09$ & 88.6 & 11.4 & 0 & 0 & $4.17 \mathrm{E}-04$ \\
\hline Os-185 & $9.40 \mathrm{E}+01$ & $3.15 \mathrm{E}-06$ & 1.2 & 81.9 & 16.8 & 0.1 & $2.00 \mathrm{E}-01$ \\
\hline Os-189m & $2.50 \mathrm{E}-01$ & $1.68 \mathrm{E}-10$ & 100 & 0 & 0 & 0 & 7.17E-06 \\
\hline Os-190m & $6.88 \mathrm{E}-03$ & $4.26 \mathrm{E}-09$ & 0 & 0 & 1.2 & 98.8 & 4.33E-06 \\
\hline Os-191 & $1.54 \mathrm{E}+01$ & $2.98 \mathrm{E}-07$ & 7.3 & 88.9 & 3.6 & 0.1 & 1.88E-02 \\
\hline Os-191m & 5.43E-01 & 2.77E-09 & 59.2 & 25.3 & 14.7 & 0.7 & $2.14 \mathrm{E}-04$ \\
\hline Os-193 & $1.25 \mathrm{E}+00$ & 1.52E-08 & 73.7 & 19.8 & 4.9 & 2.2 & 8.89E-04 \\
\hline Os-194 & $2.19 \mathrm{E}+03$ & $2.68 \mathrm{E}-05$ & 14.7 & 84.7 & 0.7 & 0 & $1.70 \mathrm{E}+00$ \\
\hline Ir-190 & $1.21 \mathrm{E}+01$ & $2.22 \mathrm{E}-07$ & 14.3 & 16.6 & 66.2 & 3.2 & 1.40E-02 \\
\hline Ir-190m & $5.00 \mathrm{E}-02$ & $7.81 \mathrm{E}-10$ & 19.3 & 2.6 & 77.7 & 0.3 & 5.62E-05 \\
\hline $\mid r-190 n$ & $1.29 \mathrm{E}-01$ & $3.41 \mathrm{E}-10$ & 0.2 & 0.1 & 40.2 & 59.5 & $1.43 \mathrm{E}-05$ \\
\hline Ir-192 & $7.40 \mathrm{E}+01$ & $8.80 \mathrm{E}-07$ & 15.5 & 25.5 & 58.6 & 0.5 & $5.57 \mathrm{E}-02$ \\
\hline $\mathrm{Ir}-192 \mathrm{~m}$ & $8.80 \mathrm{E}+04$ & $3.44 \mathrm{E}-06$ & 56.4 & 5.5 & 38.1 & 0 & $2.18 \mathrm{E}-01$ \\
\hline $\mid r-194$ & $7.98 \mathrm{E}-01$ & 1.69E-08 & 93.5 & 0 & 3.6 & 2.7 & $9.40 \mathrm{E}-04$ \\
\hline Ir-194m & $1.71 \mathrm{E}+02$ & $3.45 \mathrm{E}-06$ & 8.4 & 13.9 & 77.4 & 0.3 & $2.19 \mathrm{E}-01$ \\
\hline Pt-191 & $2.80 \mathrm{E}+00$ & 1.61E-08 & 21.9 & 24 & 45.6 & 8.8 & 9.86E-04 \\
\hline Pt-193 & $1.83 \mathrm{E}+04$ & 1.92E-08 & 6.5 & 87.5 & 6.1 & 0 & $1.22 \mathrm{E}-03$ \\
\hline Pt-193m & $4.33 E+00$ & 1.35E-08 & 36.2 & 60.1 & 3.5 & 0.4 & $8.36 \mathrm{E}-04$ \\
\hline Pt-195m & $4.02 \mathrm{E}+00$ & 1.99E-08 & 35.5 & 48.9 & 14 & 1.6 & $1.23 \mathrm{E}-03$ \\
\hline Pt-197 & $7.63 \mathrm{E}-01$ & $3.46 \mathrm{E}-09$ & 88.2 & 3.8 & 4.9 & 3.3 & $1.92 \mathrm{E}-04$ \\
\hline Pt-197m & 6.56E-02 & 1.10E-09 & 61.5 & 0.1 & 5.5 & 33 & $3.23 \mathrm{E}-05$ \\
\hline $\mathrm{Au}-194$ & $1.65 \mathrm{E}+00$ & 2.84E-08 & 22.7 & 7.1 & 50 & 20.1 & $1.69 \mathrm{E}-03$ \\
\hline $\mathrm{Au}-195$ & $1.83 E+02$ & $2.80 \mathrm{E}-07$ & 25.3 & 34.9 & 39.6 & 0.1 & $1.77 \mathrm{E}-02$ \\
\hline Au-195m & 3.53E-04 & $5.43 \mathrm{E}-13$ & 25 & 34.8 & 39.4 & 0.7 & 3.42E-08 \\
\hline Au-198 & $2.70 \mathrm{E}+00$ & $2.64 \mathrm{E}-08$ & 40.2 & 16 & 36.1 & 7.7 & $1.61 \mathrm{E}-03$ \\
\hline Au-198m & $2.30 \mathrm{E}+00$ & 4.57E-08 & 63 & 19.1 & 17.8 & 0 & 2.83E-03 \\
\hline Au-199 & $3.14 \mathrm{E}+00$ & $1.58 \mathrm{E}-08$ & 55.9 & 24.7 & 16.6 & 2.9 & $9.70 \mathrm{E}-04$ \\
\hline
\end{tabular}


Table 5. Effective Dose for Annual Average Releases (1 TBq/a) of fusion activation products (continued)

\begin{tabular}{|c|c|c|c|c|c|c|c|}
\hline Nuclide & $\begin{array}{c}\text { Nuclide } \\
\text { Half-life } \\
\text { (days) }\end{array}$ & $\begin{array}{l}\text { MEI 2-km } \\
\text { total EDE } \\
\text { (Sv/year) }\end{array}$ & $\begin{array}{l}\text { Inhalation } \\
\% \text { of } \\
\text { total dose }\end{array}$ & $\begin{array}{l}\text { Ingestion } \\
\% \text { of } \\
\text { total dose }\end{array}$ & $\begin{array}{c}\text { Ground } \\
\text { surfaces } \\
\% \text { of } \\
\text { total dose }\end{array}$ & $\begin{array}{c}\text { Air } \\
\text { immersion } \\
\% \text { of } \\
\text { total dose }\end{array}$ & $\begin{array}{c}2-100 \mathrm{~km} \\
\text { population } \\
\text { dose } \\
\text { (person- } \\
\text { Sv/year) }\end{array}$ \\
\hline $\mathrm{Hg}-194$ & $9.49 \mathrm{E}+04$ & $1.87 \mathrm{E}-05$ & 1.3 & 87.2 & 11.6 & 0 & $1.19 \mathrm{E}+00$ \\
\hline $\mathrm{Hg}-197$ & $2.67 \mathrm{E}+00$ & $7.55 \mathrm{E}-09$ & 46 & 26.6 & 23.3 & 4.2 & $4.60 \mathrm{E}-04$ \\
\hline $\mathrm{Hg}-197 \mathrm{~m}$ & $9.92 \mathrm{E}-01$ & 8.65E-09 & 67.9 & 9.8 & 17 & 5.3 & $5.11 \mathrm{E}-04$ \\
\hline $\mathrm{Hg}-199 \mathrm{~m}$ & $2.96 \mathrm{E}-02$ & $1.42 \mathrm{E}-10$ & 100 & 0 & 0 & 0 & $1.23 \mathrm{E}-06$ \\
\hline $\mathrm{Hg}-203$ & $4.66 \mathrm{E}+01$ & $9.82 \mathrm{E}-07$ & 2.7 & 87.4 & 9.8 & 0.1 & $6.21 \mathrm{E}-02$ \\
\hline TI-200 & $1.09 \mathrm{E}+00$ & $2.15 \mathrm{E}-08$ & 12.6 & 2 & 54 & 31.4 & $1.24 \mathrm{E}-03$ \\
\hline TI-201 & $3.04 \mathrm{E}+00$ & $5.23 \mathrm{E}-09$ & 25.8 & 14.7 & 51.2 & 8.3 & $3.21 \mathrm{E}-04$ \\
\hline TI-202 & $1.22 \mathrm{E}+01$ & $8.40 \mathrm{E}-08$ & 6.9 & 30.5 & 59.9 & 2.8 & $5.27 \mathrm{E}-03$ \\
\hline TI-204 & $1.38 \mathrm{E}+03$ & $1.03 \mathrm{E}-06$ & 1.3 & 98.1 & 0.2 & 0 & $6.51 \mathrm{E}-02$ \\
\hline TI-206 & $2.92 \mathrm{E}-03$ & $1.07 \mathrm{E}-11$ & 100 & 0 & 0 & 0 & $4.75 \mathrm{E}-10$ \\
\hline TI-207 & $3.31 \mathrm{E}-03$ & $2.98 \mathrm{E}-12$ & 0 & 0 & 0.5 & 99.7 & $2.53 \mathrm{E}-10$ \\
\hline TI-208 & $2.12 \mathrm{E}-03$ & $2.43 \mathrm{E}-09$ & 0 & 0 & 0.3 & 100 & $1.41 \mathrm{E}-08$ \\
\hline TI-209 & $1.53 \mathrm{E}-03$ & $6.06 \mathrm{E}-10$ & 0.9 & 0 & 0.2 & 98.8 & $1.88 \mathrm{E}-07$ \\
\hline TI-210 & $9.03 \mathrm{E}-04$ & $1.27 \mathrm{E}-10$ & 6.6 & 9 & 0.1 & 84.3 & $3.30 \mathrm{E}-06$ \\
\hline $\mathrm{Pb}-202$ & $1.10 \mathrm{E}+08$ & 4.60E-06 & 12.7 & 65.7 & 21.5 & 0 & $2.92 \mathrm{E}-01$ \\
\hline $\mathrm{Pb}-203$ & $2.17 \mathrm{E}+00$ & $1.20 \mathrm{E}-08$ & 25.5 & 11.7 & 50.1 & 12.5 & $7.22 \mathrm{E}-04$ \\
\hline $\mathrm{Pb}-205$ & $5.22 \mathrm{E}+09$ & $1.38 \mathrm{E}-07$ & 15.8 & 82.6 & 1.2 & 0 & $8.73 \mathrm{E}-03$ \\
\hline Pb-209 & $1.36 \mathrm{E}-01$ & $5.13 \mathrm{E}-10$ & 100 & 0 & 0 & 0 & $1.65 \mathrm{E}-05$ \\
\hline $\mathrm{Pb}-210$ & $8.13 E+03$ & $4.71 \mathrm{E}-04$ & 16.3 & 83.7 & 0 & 0 & $2.99 \mathrm{E}+01$ \\
\hline Pb-211 & $2.51 \mathrm{E}-02$ & $3.99 \mathrm{E}-08$ & 99 & 0 & 0 & 1.1 & $2.95 \mathrm{E}-04$ \\
\hline Pb-212 & $4.43 \mathrm{E}-01$ & $9.50 \mathrm{E}-07$ & 99.3 & 0 & 0.6 & 0.1 & 5.19E-02 \\
\hline $\mathrm{Pb}-214$ & $1.86 \mathrm{E}-02$ & $4.26 \mathrm{E}-08$ & 92.5 & 0 & 0.6 & 7 & 4.77E-04 \\
\hline Bi-206 & $6.24 \mathrm{E}+00$ & $2.53 \mathrm{E}-07$ & 13.7 & 13.6 & 66 & 6.8 & $1.58 \mathrm{E}-02$ \\
\hline $\mathrm{Bi}-207$ & $1.39 \mathrm{E}+04$ & 3.62E-06 & 2.3 & 10.4 & 87 & 0.2 & $2.29 \mathrm{E}-01$ \\
\hline $\mathrm{Bi}-210$ & $5.01 \mathrm{E}+00$ & $1.75 \mathrm{E}-06$ & 64 & 36.2 & 0 & 0 & 1.12E-01 \\
\hline Bi-210m & $1.10 \mathrm{E}+09$ & 5.09E-05 & 86.8 & 13.1 & 0 & 0 & $3.22 \mathrm{E}+00$ \\
\hline $\mathrm{Bi}-211$ & $1.48 \mathrm{E}-03$ & $1.90 \mathrm{E}-11$ & 26.3 & 1.9 & 0.4 & 71.6 & $2.58 \mathrm{E}-07$ \\
\hline Bi-212 & $4.21 \mathrm{E}-02$ & 9.72E-08 & 92.7 & 0 & 0.4 & 6.9 & $1.23 \mathrm{E}-03$ \\
\hline $\mathrm{Bi}-213$ & 3.17E-02 & $7.25 \mathrm{E}-08$ & 98.9 & 0 & 0 & 1.1 & $6.85 \mathrm{E}-04$ \\
\hline $\mathrm{Bi}-214$ & $1.38 \mathrm{E}-02$ & $3.10 \mathrm{E}-08$ & 80 & 0.6 & 0.4 & 19 & 1.57E-04 \\
\hline Po-210 & $1.38 \mathrm{E}+02$ & 1.32E-04 & 36.1 & 63.6 & 0 & 0 & $8.35 \mathrm{E}+00$ \\
\hline Po-211 & 5.97E-06 & $5.39 \mathrm{E}-15$ & 0 & 0 & 0.5 & 99.4 & $4.58 \mathrm{E}-13$ \\
\hline Po-213 & $4.86 \mathrm{E}-11$ & 1.84E-19 & 100 & 0 & 0 & 0 & $5.90 \mathrm{E}-15$ \\
\hline Po-214 & 1.90E-09 & $1.07 \mathrm{E}-16$ & 16.6 & 83.5 & 0 & 0 & $6.80 \mathrm{E}-12$ \\
\hline Po-215 & 2.06E-08 & $3.27 \mathrm{E}-14$ & 99.1 & 0 & 0 & 1.1 & $2.43 \mathrm{E}-10$ \\
\hline Po-216 & 1.69E-06 & 3.62E-12 & 99.4 & 0 & 0.6 & 0.1 & $1.98 \mathrm{E}-07$ \\
\hline Po-218 & $2.12 \mathrm{E}-03$ & 4.24E-09 & 93.4 & 0 & 0.7 & 5.8 & $5.78 \mathrm{E}-05$ \\
\hline At-211 & 3.01E-01 & $4.20 \mathrm{E}-07$ & 92.6 & 6.8 & 0.5 & 0.1 & $1.91 \mathrm{E}-02$ \\
\hline At-217 & 3.74E-07 & $8.55 \mathrm{E}-13$ & 98.8 & 0 & 0 & 1.1 & 8.07E-09 \\
\hline Rn-218 & 4.05E-08 & $2.29 \mathrm{E}-15$ & 16.7 & 83.4 & 0 & 0 & $1.45 \mathrm{E}-10$ \\
\hline Rn-219 & $4.58 \mathrm{E}-05$ & 7.31E-11 & 98.9 & 0 & 0 & 1.1 & $5.42 \mathrm{E}-07$ \\
\hline Rn-220 & $6.44 \mathrm{E}-04$ & 1.38E-09 & 99.3 & 0 & 0.6 & 0.1 & 7.56E-05 \\
\hline $\mathrm{Rn}-222$ & $3.82 \mathrm{E}+00$ & $6.40 \mathrm{E}-08$ & 8.7 & 8 & 82.8 & 0.4 & 8.42E-03 \\
\hline Fr-221 & $3.33 \mathrm{E}-03$ & $5.99 \mathrm{E}-09$ & 98.2 & 0 & 0.1 & 1.8 & 8.02E-05 \\
\hline
\end{tabular}


Table 5. Effective Dose for Annual Average Releases (1 TBq/a) of fusion activation products (continued)

\begin{tabular}{|c|c|c|c|c|c|c|c|}
\hline Nuclide & $\begin{array}{c}\text { Nuclide } \\
\text { Half-life } \\
\text { (days) }\end{array}$ & $\begin{array}{l}\text { MEI 2-km } \\
\text { total EDE } \\
\text { (Sv/year) }\end{array}$ & $\begin{array}{l}\text { Inhalation } \\
\% \text { of } \\
\text { total dose }\end{array}$ & $\begin{array}{l}\text { Ingestion } \\
\% \text { of } \\
\text { total dose }\end{array}$ & $\begin{array}{c}\text { Ground } \\
\text { surfaces } \\
\% \text { of } \\
\text { total dose }\end{array}$ & $\begin{array}{c}\text { Air } \\
\text { immersion } \\
\% \text { of } \\
\text { total dose }\end{array}$ & $\begin{array}{c}2-100 \mathrm{~km} \\
\text { population } \\
\text { dose } \\
\text { (person- } \\
\text { Sv/year) }\end{array}$ \\
\hline Fr-223 & $1.51 \mathrm{E}-02$ & $1.80 \mathrm{E}-10$ & 1.1 & 0 & 3.1 & 95.6 & $1.18 \mathrm{E}-06$ \\
\hline Ra-222 & $3.82 \mathrm{E}+00$ & $4.73 \mathrm{E}-08$ & 0.1 & 99.2 & 0.7 & 0.1 & $3.06 \mathrm{E}-03$ \\
\hline Ra-223 & $1.14 \mathrm{E}+01$ & $4.82 \mathrm{E}-05$ & 91.7 & 8.3 & 0.1 & 0 & $3.03 E+00$ \\
\hline Ra-224 & $3.62 \mathrm{E}+00$ & $1.80 \mathrm{E}-05$ & 95 & 4.8 & 0.2 & 0 & $1.12 \mathrm{E}+00$ \\
\hline Ra-225 & $1.48 \mathrm{E}+01$ & $4.72 \mathrm{E}-05$ & 93.6 & 6.3 & 0.1 & 0 & $3.00 \mathrm{E}+00$ \\
\hline Ra-226 & $5.84 \mathrm{E}+05$ & 1.32E-04 & 35.2 & 62.4 & 2.6 & 0 & $8.41 \mathrm{E}+00$ \\
\hline Ra-228 & $2.10 \mathrm{E}+03$ & $1.15 \mathrm{E}-04$ & 21.6 & 77.2 & 1.6 & 0 & $7.35 \mathrm{E}+00$ \\
\hline Ac-225 & $1.00 \mathrm{E}+01$ & $4.77 \mathrm{E}-05$ & 98.7 & 1.2 & 0 & 0 & $3.00 \mathrm{E}+00$ \\
\hline Ac-227 & $7.95 \mathrm{E}+03$ & $8.03 \mathrm{E}-03$ & 88 & 12 & 0 & 0 & $5.10 \mathrm{E}+02$ \\
\hline Ac-228 & $2.55 \mathrm{E}-01$ & $6.59 \mathrm{E}-07$ & 98.3 & 0.6 & 0.3 & 0.7 & $4.21 \mathrm{E}-02$ \\
\hline Th-226 & $2.15 \mathrm{E}-02$ & $1.68 \mathrm{E}-07$ & 100 & 0 & 0 & 0 & $1.03 \mathrm{E}-03$ \\
\hline Th-227 & $1.87 \mathrm{E}+01$ & $9.54 \mathrm{E}-05$ & 98.8 & 1.1 & 0.1 & 0 & $6.04 \mathrm{E}+00$ \\
\hline Th-228 & $6.98 \mathrm{E}+02$ & 1.86E-03 & 98.4 & 1.6 & 0.1 & 0 & $1.18 \mathrm{E}+02$ \\
\hline Th-229 & $2.68 \mathrm{E}+06$ & $1.03 \mathrm{E}-02$ & 97.1 & 2.4 & 0 & 0 & $6.51 \mathrm{E}+02$ \\
\hline Th-230 & $2.81 \mathrm{E}+07$ & $1.57 \mathrm{E}-03$ & 97.5 & 2.3 & 0 & 0 & $9.94 \mathrm{E}+01$ \\
\hline Th-231 & $1.06 \mathrm{E}+00$ & $4.76 \mathrm{E}-09$ & 95 & 0.3 & 3.5 & 1.2 & $2.76 \mathrm{E}-04$ \\
\hline Th-232 & $5.13 \mathrm{E}+12$ & $6.67 \mathrm{E}-03$ & 97.2 & 2.9 & 0 & 0 & $4.24 \mathrm{E}+02$ \\
\hline Th-234 & $2.41 \mathrm{E}+01$ & $3.96 \mathrm{E}-07$ & 49 & 50.3 & 0.7 & 0 & $2.50 \mathrm{E}-02$ \\
\hline Pa-230 & $1.74 \mathrm{E}+01$ & $9.12 \mathrm{E}-06$ & 96.9 & 2 & 1.1 & 0 & $5.79 \mathrm{E}-01$ \\
\hline Pa-231 & $1.36 \mathrm{E}+07$ & $5.83 \mathrm{E}-03$ & 87 & 13 & 0 & 0 & $3.70 \mathrm{E}+02$ \\
\hline $\mathrm{Pa}-232$ & $1.31 \mathrm{E}+00$ & $4.03 \mathrm{E}-07$ & 99.3 & 0.6 & 0 & 0 & $2.46 \mathrm{E}-02$ \\
\hline Pa-233 & $2.70 \mathrm{E}+01$ & 1.62E-07 & 31.3 & 35.2 & 32.5 & 0.7 & $1.02 \mathrm{E}-02$ \\
\hline Pa-234 & $2.79 \mathrm{E}-01$ & $1.88 \mathrm{E}-08$ & 22.8 & 0 & 23.7 & 53.2 & $8.34 \mathrm{E}-04$ \\
\hline Pa-234m & $8.13 \mathrm{E}-04$ & $2.55 \mathrm{E}-13$ & 2.7 & 0 & 0.1 & 97.3 & $4.53 \mathrm{E}-10$ \\
\hline $\mathrm{U}-230$ & $2.08 \mathrm{E}+01$ & 1.30E-04 & 90.8 & 9 & 0 & 0 & $8.18 \mathrm{E}+00$ \\
\hline U-231 & $4.20 \mathrm{E}+00$ & $1.33 \mathrm{E}-08$ & 48.7 & 25.8 & 22.6 & 2.6 & $8.22 \mathrm{E}-04$ \\
\hline U-232 & $2.63 E+04$ & 4.05E-03 & 97.5 & 2.5 & 0 & 0 & $2.57 \mathrm{E}+02$ \\
\hline $\mathrm{U}-233$ & $5.81 \mathrm{E}+07$ & $7.87 \mathrm{E}-04$ & 97.3 & 2.6 & 0 & 0 & $4.99 \mathrm{E}+01$ \\
\hline U-234 & $8.92 \mathrm{E}+07$ & $7.86 \mathrm{E}-04$ & 97.5 & 2.5 & 0 & 0 & $4.98 \mathrm{E}+01$ \\
\hline U-235 & $2.57 \mathrm{E}+11$ & $7.27 \mathrm{E}-04$ & 97.2 & 2.6 & 0.1 & 0 & $4.61 \mathrm{E}+01$ \\
\hline U-236 & $8.55 \mathrm{E}+09$ & $7.26 \mathrm{E}-04$ & 97.4 & 2.6 & 0 & 0 & $4.61 \mathrm{E}+01$ \\
\hline U-237 & $6.75 \mathrm{E}+00$ & $4.12 \mathrm{E}-08$ & 47.1 & 29.4 & 21.8 & 1.6 & $2.57 \mathrm{E}-03$ \\
\hline U-238 & $1.63 \mathrm{E}+12$ & $7.25 \mathrm{E}-04$ & 97.5 & 2.5 & 0 & 0 & $4.60 \mathrm{E}+01$ \\
\hline U-239 & 1.64E-02 & $3.88 \mathrm{E}-10$ & 43.6 & 2.1 & 8.2 & 46.1 & 1.04E-05 \\
\hline U-240 & $5.88 \mathrm{E}-01$ & 1.49E-08 & 82.6 & 0.1 & 11 & 6.6 & 8.37E-04 \\
\hline $\mathrm{Np}-235$ & $3.96 \mathrm{E}+02$ & $4.28 \mathrm{E}-08$ & 52.3 & 30.4 & 17.2 & 0 & $2.71 \mathrm{E}-03$ \\
\hline Np-236a & $4.20 \mathrm{E}+07$ & $6.41 \mathrm{E}-04$ & 91 & 9 & 0.1 & 0 & $4.06 \mathrm{E}+01$ \\
\hline $\mathrm{Np}-236 \mathrm{~b}$ & $9.38 \mathrm{E}-01$ & $4.21 \mathrm{E}-07$ & 99.3 & 0.6 & 0.1 & 0.1 & 2.61E-02 \\
\hline $\mathrm{Np}-237$ & $7.81 \mathrm{E}+08$ & $3.17 \mathrm{E}-03$ & 91.2 & 9 & 0 & 0 & $2.01 \mathrm{E}+02$ \\
\hline $\mathrm{Np}-238$ & $2.12 \mathrm{E}+00$ & $2.00 \mathrm{E}-07$ & 91.5 & 2.8 & 4.7 & 1.5 & $1.25 \mathrm{E}-02$ \\
\hline $\mathrm{Np}-239$ & $2.36 \mathrm{E}+00$ & $2.21 \mathrm{E}-08$ & 58.4 & 20.6 & 16.9 & 3.8 & $1.34 \mathrm{E}-03$ \\
\hline $\mathrm{Np}-240$ & 4.51E-02 & $6.15 \mathrm{E}-09$ & 5.5 & 0 & 6.5 & 88.1 & 8.43E-05 \\
\hline $\mathrm{Np}-240 \mathrm{~m}$ & 5.14E-03 & $7.21 \mathrm{E}-10$ & 0.3 & 0 & 0.8 & 98.9 & $6.11 \mathrm{E}-07$ \\
\hline Pu-236 & $1.04 \mathrm{E}+03$ & 7.84E-04 & 97.7 & 2.3 & 0 & 0 & $4.97 E+01$ \\
\hline
\end{tabular}


Table 5. Effective Dose for Annual Average Releases (1 TBq/a) of fusion activation products (continued)

\begin{tabular}{|c|c|c|c|c|c|c|c|}
\hline Nuclide & $\begin{array}{c}\text { Nuclide } \\
\text { Half-life } \\
\text { (days) }\end{array}$ & $\begin{array}{l}\text { MEI 2-km } \\
\text { total EDE } \\
\text { (Sv/year) }\end{array}$ & $\begin{array}{l}\text { Inhalation } \\
\% \text { of } \\
\text { total dose } \\
\end{array}$ & $\begin{array}{l}\text { Ingestion } \\
\% \text { of } \\
\text { total dose } \\
\end{array}$ & $\begin{array}{c}\text { Ground } \\
\text { surfaces } \\
\% \text { of } \\
\text { total dose } \\
\end{array}$ & $\begin{array}{c}\text { Air } \\
\text { immersion } \\
\% \text { of } \\
\text { total dose } \\
\end{array}$ & $\begin{array}{c}2-100 \mathrm{~km} \\
\text { population } \\
\text { dose } \\
\text { (person- } \\
\text { Sv/year) } \\
\end{array}$ \\
\hline Pu-237 & $4.53 \mathrm{E}+01$ & $4.26 \mathrm{E}-08$ & 22.1 & 25.4 & 51.9 & 0.6 & $2.70 \mathrm{E}-03$ \\
\hline Pu-238 & $3.20 \mathrm{E}+04$ & $1.82 \mathrm{E}-03$ & 97.3 & 3 & 0 & 0 & $1.16 \mathrm{E}+02$ \\
\hline Pu-239 & $8.81 \mathrm{E}+06$ & $2.01 \mathrm{E}-03$ & 96.5 & 3.1 & 0 & 0 & $1.27 \mathrm{E}+02$ \\
\hline Pu-240 & $2.40 \mathrm{E}+06$ & $2.01 \mathrm{E}-03$ & 96.5 & 3.1 & 0 & 0 & $1.27 \mathrm{E}+02$ \\
\hline Pu-240 & $2.40 \mathrm{E}+06$ & $2.01 \mathrm{E}-03$ & 96.5 & 3.1 & 0 & 0 & $1.27 \mathrm{E}+02$ \\
\hline Pu-241 & $5.26 \mathrm{E}+03$ & $3.48 \mathrm{E}-05$ & 96.6 & 3.6 & 0 & 0 & $2.21 \mathrm{E}+00$ \\
\hline Pu-242 & $1.37 \mathrm{E}+08$ & $1.89 \mathrm{E}-03$ & 96.8 & 3.1 & 0 & 0 & $1.20 \mathrm{E}+02$ \\
\hline Pu-243 & $2.07 \mathrm{E}-01$ & $9.76 \mathrm{E}-10$ & 83.2 & 0.3 & 4.9 & 11.7 & $4.43 \mathrm{E}-05$ \\
\hline Pu-244 & $3.02 \mathrm{E}+10$ & $1.88 \mathrm{E}-03$ & 97.3 & 3 & 0 & 0 & $1.20 \mathrm{E}+02$ \\
\hline Pu-245 & $4.38 \mathrm{E}-01$ & $1.11 \mathrm{E}-08$ & 63.2 & 1.9 & 15.2 & 19.3 & 5.77E-04 \\
\hline Pu-246 & $1.09 \mathrm{E}+01$ & 9.67E-08 & 0 & 0.4 & 98.2 & 1.3 & $6.38 \mathrm{E}-03$ \\
\hline Am-241 & $1.58 \mathrm{E}+05$ & $3.20 \mathrm{E}-03$ & 95.6 & 4.3 & 0 & 0 & $2.03 \mathrm{E}+02$ \\
\hline Am-242 & $6.68 \mathrm{E}-01$ & $3.61 \mathrm{E}-07$ & 99.4 & 0.5 & 0 & 0 & $2.27 \mathrm{E}-02$ \\
\hline Am-242m & $5.55 \mathrm{E}+04$ & $3.13 \mathrm{E}-03$ & 95.8 & 4.1 & 0 & 0 & $1.99 \mathrm{E}+02$ \\
\hline Am-243 & $2.69 \mathrm{E}+06$ & $3.20 \mathrm{E}-03$ & 95.6 & 4.3 & 0 & 0 & $2.03 \mathrm{E}+02$ \\
\hline Am-244 & $4.21 \mathrm{E}-01$ & $1.08 \mathrm{E}-07$ & 92.6 & 1.1 & 2.6 & 3.8 & $6.80 \mathrm{E}-03$ \\
\hline Am-245 & $8.54 \mathrm{E}-02$ & $5.47 \mathrm{E}-10$ & 68.4 & 0 & 4.4 & 27.4 & $1.65 \mathrm{E}-05$ \\
\hline Am-246 & $2.71 \mathrm{E}-02$ & $4.78 \mathrm{E}-09$ & 5.3 & 0 & 3.8 & 91 & $4.08 \mathrm{E}-05$ \\
\hline $\mathrm{Cm}-242$ & $1.63 \mathrm{E}+02$ & $1.03 \mathrm{E}-04$ & 97.1 & 2.6 & 0 & 0 & $6.52 \mathrm{E}+00$ \\
\hline $\mathrm{Cm}-243$ & $1.04 \mathrm{E}+04$ & $2.15 \mathrm{E}-03$ & 95.8 & 4.1 & 0 & 0 & $1.36 \mathrm{E}+02$ \\
\hline $\mathrm{Cm}-244$ & $6.61 \mathrm{E}+03$ & $1.66 \mathrm{E}-03$ & 95.8 & 4.2 & 0 & 0 & $1.05 \mathrm{E}+02$ \\
\hline $\mathrm{Cm}-245$ & $3.10 \mathrm{E}+06$ & $3.32 \mathrm{E}-03$ & 95.8 & 4.1 & 0 & 0 & $2.10 \mathrm{E}+02$ \\
\hline $\mathrm{Cm}-246$ & $1.73 \mathrm{E}+06$ & $3.32 \mathrm{E}-03$ & 95.8 & 4.1 & 0 & 0 & $2.10 \mathrm{E}+02$ \\
\hline $\mathrm{Cm}-247$ & $5.69 \mathrm{E}+09$ & $3.01 \mathrm{E}-03$ & 96 & 4.1 & 0 & 0 & $1.91 \mathrm{E}+02$ \\
\hline $\mathrm{Cm}-248$ & $1.24 \mathrm{E}+08$ & $1.17 \mathrm{E}-02$ & 95.7 & 4.2 & 0 & 0 & $7.42 \mathrm{E}+02$ \\
\hline $\mathrm{Cm}-249$ & $4.46 \mathrm{E}-02$ & $1.38 \mathrm{E}-09$ & 92 & 1.2 & 0.5 & 6.3 & $7.39 \mathrm{E}-05$ \\
\hline $\mathrm{Cm}-250$ & $2.52 \mathrm{E}+06$ & 1.73E-06 & 0.1 & 21.8 & 78 & 0 & 1.11E-01 \\
\hline Bk-249 & $3.20 \mathrm{E}+02$ & $8.06 \mathrm{E}-06$ & 95 & 4.9 & 0 & 0 & $5.11 \mathrm{E}-01$ \\
\hline Bk-250 & 1.34E-01 & 4.67E-08 & 86.5 & 1.9 & 2 & 9.7 & $2.51 \mathrm{E}-03$ \\
\hline Cf-248 & $3.34 \mathrm{E}+02$ & $2.70 \mathrm{E}-04$ & 93.7 & 6.3 & 0 & 0 & $1.71 \mathrm{E}+01$ \\
\hline Cf-249 & $1.28 \mathrm{E}+05$ & $2.44 \mathrm{E}-03$ & 86.9 & 12.9 & 0 & 0 & $1.54 \mathrm{E}+02$ \\
\hline Cf-250 & $4.77 \mathrm{E}+03$ & $1.25 \mathrm{E}-03$ & 89.6 & 10.3 & 0 & 0 & $7.92 \mathrm{E}+01$ \\
\hline Cf-251 & $3.29 \mathrm{E}+05$ & $2.50 \mathrm{E}-03$ & 87.2 & 12.6 & 0 & 0 & $1.58 \mathrm{E}+02$ \\
\hline Cf-252 & $9.63 \mathrm{E}+02$ & $8.27 \mathrm{E}-04$ & 92.6 & 7.4 & 0 & 0 & $5.24 \mathrm{E}+01$ \\
\hline Cf-253 & $1.78 \mathrm{E}+01$ & 1.78E-05 & 99.4 & 0.8 & 0 & 0 & $1.13 \mathrm{E}+00$ \\
\hline Cf-254 & $6.05 \mathrm{E}+01$ & 1.74E-03 & 94.8 & 4.9 & 0 & 0 & $1.10 \mathrm{E}+02$ \\
\hline Es-253 & $2.05 \mathrm{E}+01$ & $1.98 \mathrm{E}-05$ & 98 & 1.6 & 0 & 0 & $1.25 \mathrm{E}+00$ \\
\hline Es-254 & $2.76 \mathrm{E}+02$ & $2.22 \mathrm{E}-04$ & 95.5 & 4.1 & 0.5 & 0 & $1.41 \mathrm{E}+01$ \\
\hline Es-254m & $1.64 \mathrm{E}+00$ & 2.81E-06 & 98.6 & 0.9 & 0.3 & 0.1 & 1.76E-01 \\
\hline Fm-254 & $1.35 \mathrm{E}-01$ & $2.81 \mathrm{E}-07$ & 99.6 & 0.3 & 0 & 0 & $1.00 \mathrm{E}-02$ \\
\hline Fm-255 & $8.36 \mathrm{E}-01$ & 1.35E-06 & 100 & 0.3 & 0 & 0 & 7.57E-02 \\
\hline
\end{tabular}




\section{CONCLUSIONS}

Radiological dose calculations have been performed for accidental atmospheric releases of 535 fusion radionuclides. The resulting tables of one terabecquerel dose values per radionuclide can be used for scoping calculations on fusion conceptual designs. The tables can also be used to evaluate design options for a given fusion facility. These results should have longevity since the radiological dose code of preference, MACCS2, was used to generate the values. When MACCS2 is upgraded, then the table values may need to be revised. Averaged weather and location conditions were used to allow initial calculation of routine release doses from 565 activation products. The routine release calculations are for 100-m stacked releases.

The release of the DOE guidance on emergency planning zones means that the FSS "no evacuation" 1-rem dose criteria must be calculated using conservative atmospheric dispersion conditions of Class $\mathrm{F}$ atmospheric stability and $1 \mathrm{~m} / \mathrm{s}$ wind speed rather than the best estimate weather that was initially directed. These new dose calculation assumptions place new restrictions on future fusion facility designs. Future designs should use large site boundaries of $1 \mathrm{~km}$ or more, reduce on-site inventories, and use inventory segmentation, as much as possible, to meet the "no-evacuation" criteria. 


\section{REFERENCES}

1. M. L. Abbott, S. L. Harms, and A. S. Rood, Dose Calculations for Accidental Airborne Releases of ITER Activation Products, EGG-EEL-10994, Idaho National Engineering Laboratory, December 1993.

2. M. L. Abbott and D. R. Wenzel, Dose Calculations for Routine Airborne Releases of ITER Activation Products, EGG-EEL-11108, Idaho National Engineering Laboratory, May 1994.

3. M. L. Abbott, Revised Results - MACCS2 Doses for Fusion Isotopes Released to the Atmosphere Using P-G Dispersion Parameters, memo MLA-11-99 to D. Petti, Idaho National Engineering and Environmental Laboratory, April 14, 1999.

4. D. I. Chanin and M. L. Young, Code Manual for MACCS2: Volume 1, User's Guide, SAND97-0594, Sandia National Laboratories, March 1997.

5. J. M. East and K. R. O'Kula, "A Comparative Study of Worker and General Public Risks from Nuclear Facility Operation using MACCS2," Proceedings of the International Conference on Mathematics and Computations, Reactor Physics, and Environmental Analyses, Portland, Oregon, April 30 - May 4, 1995, American Nuclear Society, 1995, 1293-1298.

6. Safety of Magnetic Fusion Facilities: Requirements, DOE STD-6002-96, US Department of Energy, May 1996.

7. Facility Safety, Order DOE O 420.1A, US Department of Energy, May 20, 2002.

8. Safety of Magnetic Fusion Facilities: Guidance, DOE STD-6003-96, US Department of Energy, May 1996, section 2.2.

9. Title 10, "Energy", US Code of Federal Regulations, Part 100, Reactor Site Criteria, Section 11, 'Determination of exclusion area low population zone, and population center distance,' November 4, 2002.

10. Assumptions Used for Evaluating the Potential Radiological Consequences of a Loss of Coolant Accident for Boiling Water Reactors, Regulatory Guide 1.3, revision 2, US Nuclear Regulatory Commission, June 1974.

11. Assumptions Used for Evaluating the Potential Radiological Consequences of a Loss of Coolant Accident for Pressurized Water Reactors, Regulatory Guide 1.4, revision 2, US Nuclear Regulatory Commission, June 1974.

12. Safety Analysis and Risk Assessment Handbook, RFP-5098, part 1, Kaiser Hill Company, April 1997, Chapter 7.

13. Nonreactor Nuclear Safety Design Criteria and Explosives Safety Criteria Guide for use with DOE O 420.1, Facility Safety, DOE G 420.1-1, US DOE, March 2000.

14. Atmospheric Dispersion Models for Potential Accident Consequence Assessments at Nuclear Power Plants, Regulatory Guide 1.145, US Nuclear Regulatory Commission, revision 1 (reissue), February 1983.

15. Manual of Protective Action Guides and Protective Actions for Nuclear Incidents, EPA-400-R92-001, NTIS PB92-164763, US Environmental Protection Agency, October 1991. 
16. Emergency Management Guide, volume II, Hazards Surveys and Hazards Assessments, DOE G 151.1-1, US Department of Energy, August 1997, p. 3-15.

17. The Tokamak Fusion Test Reactor Decontamination and Decommissioning Project and the Tokamak Physics Experiment at the Princeton Plasma Physics Laboratory, DOE/EA-0813, US Department of Energy, May 1994.

18. C. G. Motloch, J. D. Levine, J. C. Commander, M. A. McKenzie-Carter, R. F. Bonney, and L. S. Masson, Preliminary Safety Analysis Report for the Tokamak Physics Experiment, INEL-95/0199, Idaho National Engineering Laboratory, April 1995.

19. National Ignition Facility Preliminary Safety Analysis Report, UCRL-ID-123759, Lawrence Livermore National Laboratory, September 1996.

20. Final Programmatic Environmental Impact Statement for Stockpile Stewardship and Management, DOE/EIS-0236, Volume III, Appendix I, "National Ignition Facility Project-Specific Analysis," US Department of Energy, September 1996.

21. Generic Models and Parameters for Assessing the Environmental Transfer of Radionuclides from Routine Releases, IAEA Safety Series No. 57, International Atomic Energy Agency, 1982.

22. J. Tadmor and Y. Gur, "Analytical Expressions for the Vertical and Lateral Dispersion Coefficients in Atmospheric Diffusion," Atmospheric Environment, 3,1969, 688 .

23. G. A. Briggs, Diffusion Estimation for Small Emissions, ATDL Contribution File No. 79, report number TID-28289, Atmospheric Turbulence and Diffusion Laboratory, National Oceanic and Atmospheric Administration, Oak Ridge, TN, May 1973. 